\title{
Control of cardiogenesis and homeostasis by cardiac fibroblasts
}

\author{
Doctoral Thesis \\ In partial fulfillment of the requirements for the degree \\ "Doctor rerum naturalium (Dr. rer. nat.)" \\ in the Molecular Medicine Study Program \\ at the Georg-August University Göttingen
}

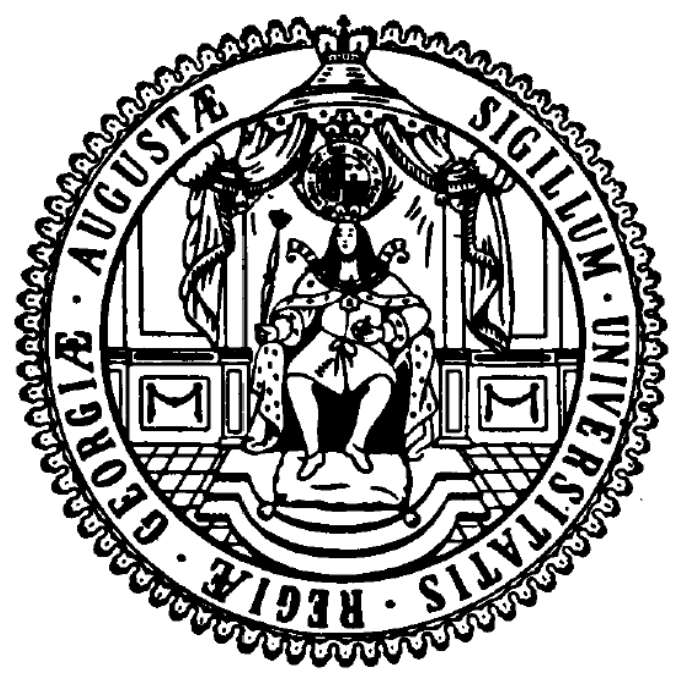

Submitted by

Sumon Sur

born in Reading, United Kingdom

Göttingen 2016 


\section{Members of the Thesis Committee:}

Prof. Dr. med. Wolfram-Hubertus Zimmermann (Supervisor)

Email: W.zimmermann@med.uni-goettingen.de

Phone: $\quad$ +49 (0) 551395787

Postal Address: $\quad$ Institute of Pharmacology and Toxicology University Medical Center Goettingen Georg-August University Göttingen Robert-Koch-Str. 40 37075 Göttingen

Germany

Prof. Dr. Dörthe Katschinski (Second member of thesis committee)

Email: doerthe.katschinski@med.uni-goettingen.de

Phone: $\quad$ +49(0) $\quad$ + 551395896

Postal Address: $\quad$ Institute of Cardiovascular Physiology Humboldtallee 23 37073 Göttingen Germany

Prof. Dr. Peter Rehling (Third member of thesis committee)

Email:_ peter.rehling@medizin.uni-goettingen.de

Phone: $\quad$ +49 (0) 551395947

Postal Address: $\quad$ Institute of Cellular Biochemistry University Medical Centre Göttingen Humboldtallee 23 37073 Göttingen Germany

Prof. Dr. Manuel Mayr (Forth member of thesis committee)

Email: $\quad$ manuel.mayr@kcl.ac.uk

Phone: + + + +44 (0) 2078485132

Postal Address: $\quad$ The James Black Centre King's College, University of London 125 Coldharbour Lane London SE5 9NU United Kingdom

Date of Disputation: 


\section{AFFIDAVIT}

Here I declare that my doctoral thesis entitled:

\section{"Control of cardiogenesis and homeostasis by cardiac fibroblasts"}

has been written independently with no other sources and aids than quoted.

Sumon Sur

Göttingen, February 2016 


\section{List of publications:}

Abonnenc M, Nabeebaccus AA, Mayr U, Barallobre-Barreiro J, Dong X, Cuello F, Sur S, Drozdov I, Langley SR, Lu R, Stathopoulou K, Didangelos A, Yin X, Zimmermann WH, Shah AM, Zampetaki A, Mayr M. 2013. Extracellular matrix secretion by cardiac fibroblasts: role of microRNA-29b and microRNA-30c. Circ Res; 113(10):1138-47.

\section{List of poster presentations:}

Sumon Sur, Peter Christalla, James Hudson, Christoph Schmidt, Doerthe Katschinski, Manuel Mayr, Wolfram-Hubertus Zimmermann (2012). Fibroblast-derived extracellular matrix controls cardiomyocyte maturation. BHF Centre of Excellence annual symposium, London, $U K$.

Sumon Sur, Peter Christalla, Angelica Roa, Wolfram H Zimmermann (2014). Influence of the Collagen Processing Heat Shock Protein 47 on Cardiomyocyte Homeostasis and Maturation. American Heart Association (AHA) Basic Cardiovascular Sciences (BCVS) 2014 Scientific Sessions, Las Vegas, Nevada, USA.

Sumon Sur, Peter Christalla, Angelica Roa, Wolfram H Zimmermann (2014).

Collagen Processing Heat Shock Protein 47 Influences Cardiomyocyte Homeostasis and Maturation. DGK Basic Science meeting 2014, Düsseldorf, Germany. 


\section{Table of Contents:}

Acknowledgements .................................................................................................................... I

Abstract..................................................................................................................................... III

List of Figures..................................................................................................................... IV

List of Tables ................................................................................................................. VI

Abbreviations ................................................................................................. VII

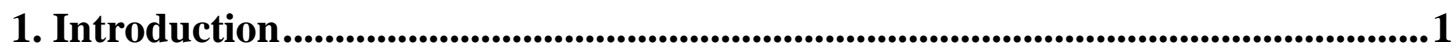

1.1 Structure and cellular components of the heart................................................ 1

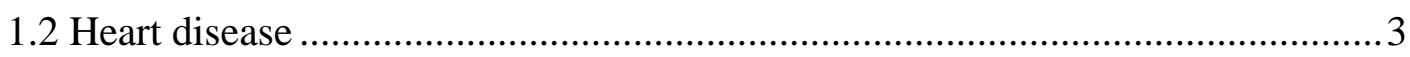

1.3 Cardiac Tissue Engineering .......................................................................

1.3.1 Engineered heart muscle (EHM) to study cell interactions ...........................5

1.4 Cardiac fibroblasts in the heart .......................................................................

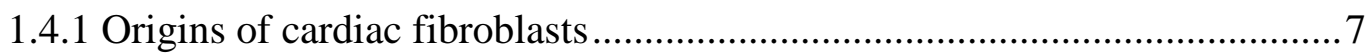

1.4.2 Function of cardiac fibroblasts in the heart................................................

1.5 Cardiac fibroblasts and the ECM .....................................................................

1.5.1 ECM remodelling in the developing and pathological heart ...................... 10

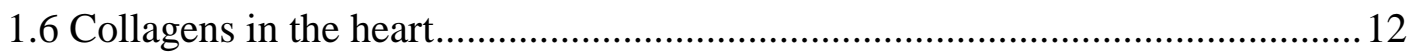

1.6.1 Structure and biosynthesis of collagen .................................................... 13

1.6.2 HSP47 in the biogenesis of collagen ...................................................... 16

1.7 Cell-ECM communication via integrin receptors .............................................. 19

1.7.1 Integrin-mediated signalling in the myocardium .......................................23

1.8 Effects of the mechanical ECM environment on cardiomyocytes......................24

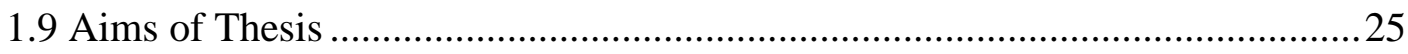

2. Materials and Methods.................................................................................................26

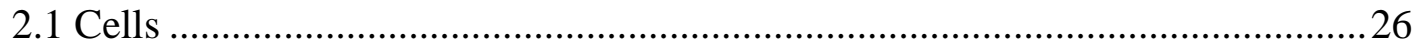

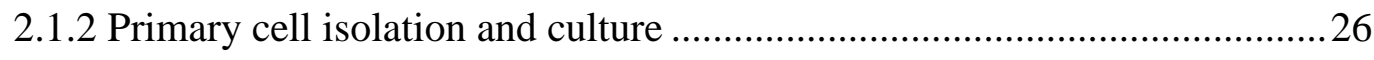

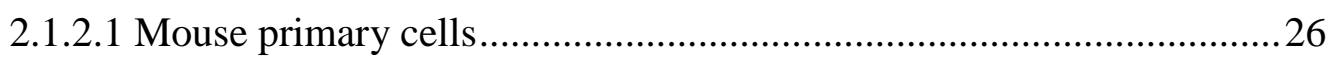

2.1.2.1.1 Mouse embryonic fibroblast isolation ...........................................26

2.1.2.1.2 Mitotic inactivation of fibroblasts with $\gamma$-irradiation .....................2

2.1.2.1.3 Preparation of inactive MEF feeder layer plates.............................2 27 
2.1.2.1.4 Neonatal mouse cardiac fibroblast isolation ...............................28

2.1.2.1.5 Passaging of primary mouse fibroblasts ...................................29

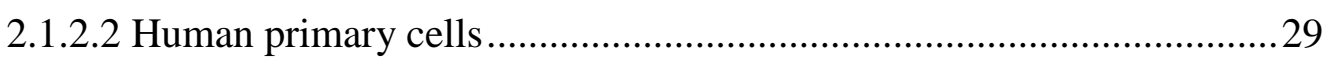

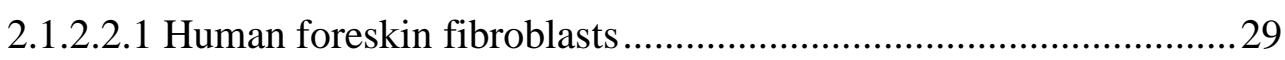

2.1.2.2.2 Human cardiac fibroblasts ........................................................29

2.1.2.2.3 Passaging and culture of primary human fibroblasts ....................30

2.1.2.3.4 Freezing and thawing of primary mouse and human fibroblasts. . 30

2.1.2.3.5 Cell count and size measurements ............................................. 30

2.1.3 Embryonic stem cell generation and culture........................................... 31

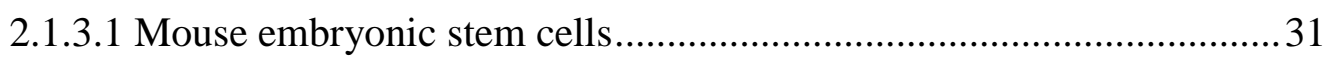

2.1.3.1.1 Culture of mouse embryonic stem cells .................................... 31

2.1.3.1.2 Scale up differentiation of mouse $\alpha$ MHC-NeoR ESCs ............... 31

2.1.3.1.3 Digestion of embryoid bodies into single cardiomyocytes ...........32

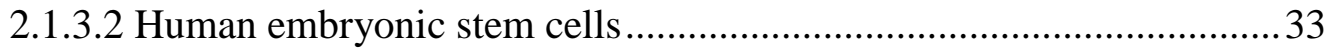

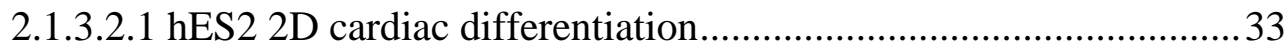

2.1.3.2.2 Single cell dissociation of hES2-cardiomyocyte monolayers....... 34

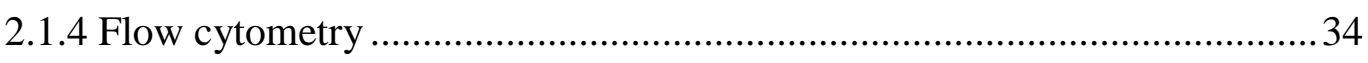

2.2 Generation of Engineered Heart Muscle (EHM) .......................................... 35

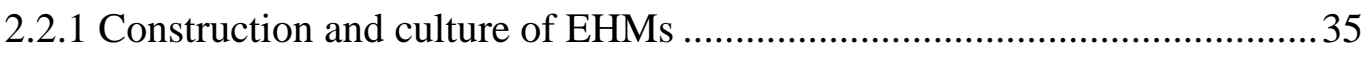

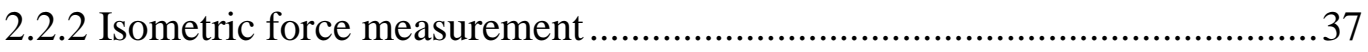

2.2.3 Whole mount Immunoflourescence imaging ............................................ 38

2.3 Integrin expression in fibroblast and cardiomyocyte populations ..................... 38

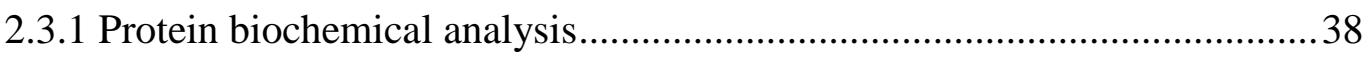

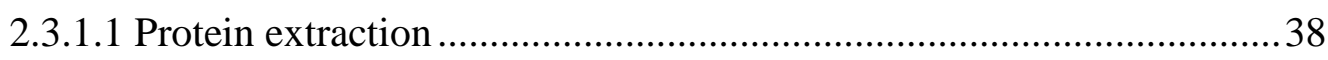

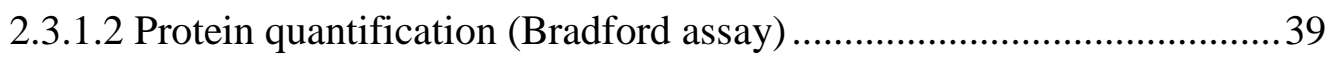

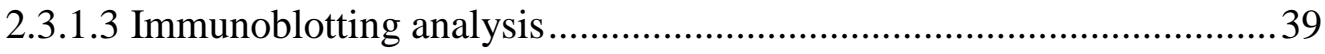

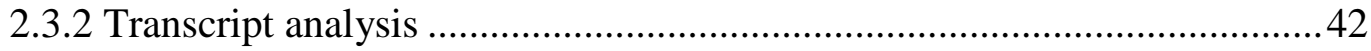

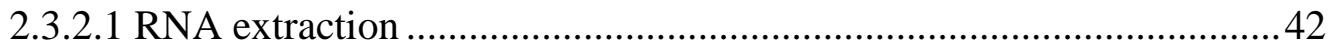

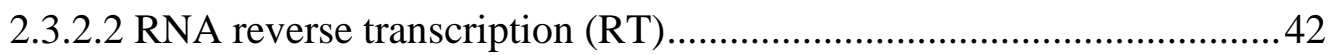

2.3.2.3 Polymerase chain reaction (PCR) …................................................ 43

2.3.2.4 Quantitative polymerase chain reaction ( $(\mathrm{PCR})$..............................4

2.3.2.5 RNA sequencing (RNA-seq) and bioinformatics ............................ 45

2.4 Assessment of integrin-ECM signalling in EHM formation ...........................46

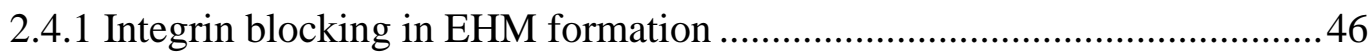


2.5 Assessment of collagen processing heat shock protein 47 knockout (HSP47

KO) MEFs

2.5.1 HSP47 KO MEFs

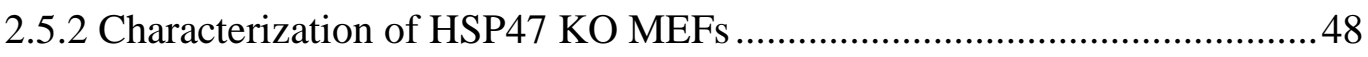

2.6 Investigating collagen secretion in HSP47 KO MEFs ...................................48

2.6.1 Assessment of soluble collagen secretion ............................................... 48

2.6.2 Deoxychelate (DOC)-separation ECM components.................................50

2.6.3 Mass spectrometric analysis of DOC-insoluble ECM components............51

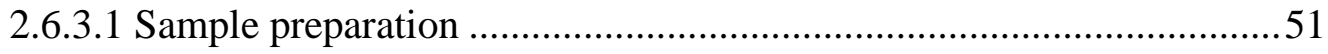

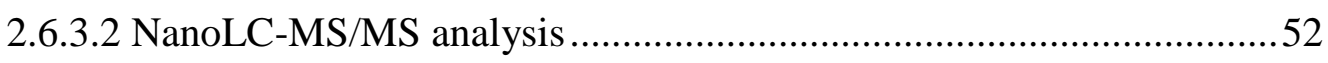

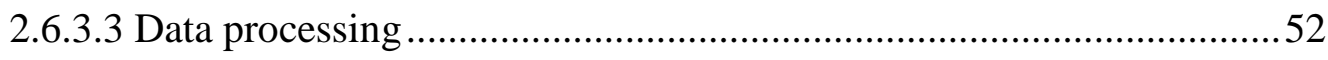

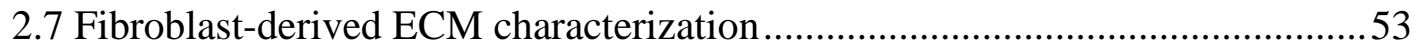

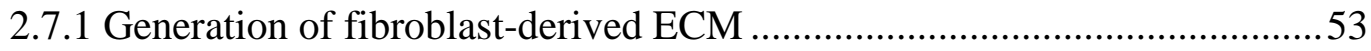

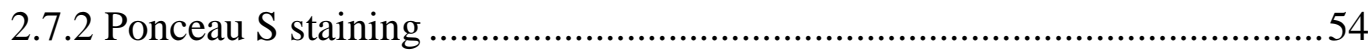

2.7.3 Seeding of purified mESC-cardiomyocytes on fibroblast-derived ECMs..55

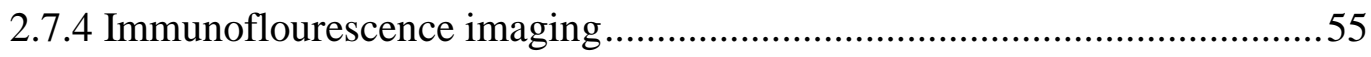

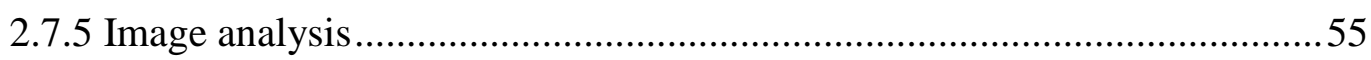

2.8 Assessment of HSP47 KO MEFs on EHM formation and functionality...........56

2.9 Generation of human HSP47 mutant cell lines ................................................56

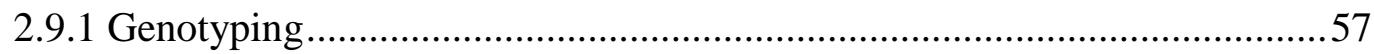

2.9.2 Characterization of SERPINH1 mutant hES2 lines .................................58

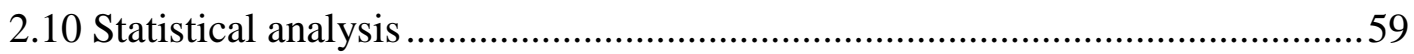

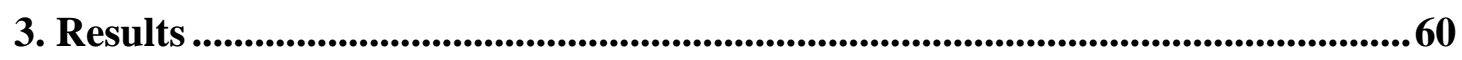

3.1 EHM as a defined model of heart muscle development ................................60

3.1.1 Cardiomyocyte differentiation and purification.......................................6 60

3.1.2 Cardiac fibroblasts are essential for EHM formation and maturation ........62

3.2 ECM-integrin crosstalk in fibroblasts is responsible in mediating tissue

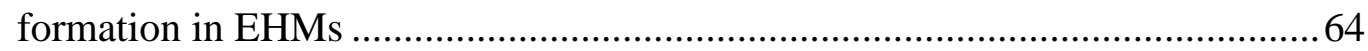

3.2.1 Collagen binding integrins are highly expressed in fibroblasts .................64

3.2.2 Blocking integrin-collagen type I interactions abolishes initial EHM

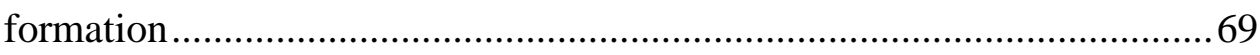

3.3 Cardiac fibroblasts construct an ECM environment in EHMs ..........................72 
3.4 Influence of collagen processing HSP47 on cardiomyocyte maturation and homeostasis.

3.4.1 Collagen processing HSP47 is highly expressed in fibroblast populations 76

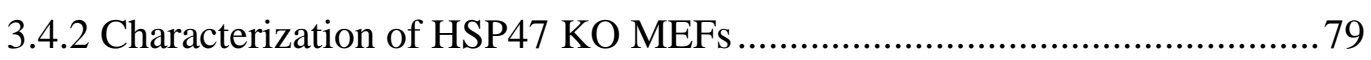

3.4.2.1 Defective Collagen type I secretion in HSP47 KO MEFs .................. 83

3.4.2.2 Deoxychelate (DOC)-separation of ECM fractions .............................. 84

3.4.2.3 Mass spectrometric analyses of ECM fractions..................................86

3.4.3 Investigation of cardio-instructive properties of fibroblast-derived ECM

3.4.3.1 Generation of fibroblast-derived ECM

3.4.3.2 Reduced ECM deposition and abnormal Collagen type I fibril formation in HSP47 KO MEFs.

3.4.3.3 Impaired CM phenotype on defective collagen processing HSP47 KO FB-derived ECMs 91

3.4.3 Collagen defective HSP47 KO MEFs impair EHM formation and functionality .92

3.4.3.1 Reduced $\operatorname{Itg} \beta 1$ integrin expression in HSP47 KO MEFs.....................95

3.4.4 Generation and validation of HSP47 KO hES2 cell line 97

4. Discussions: 100

4.1 Fibroblasts are essential for engineered heart muscle formation 101

4.2 Integrin mediated fibroblast-ECM interactions are essential for engineereed heart muscle formation

4.3 FBs secrete a cardio-instructive ECM environment important in mediating heart muscle maturation. 105

4.4 Collagen processing HSP47 is an important regulator of heart muscle formation and maturation 107

4.4.1 HSP47 is highly expressed in fibroblasts 107

4.4.2 Absence of HSP47 in mouse fibroblasts impairs ECM Collagen type I secretion and fibrillar organisation

4.4.3 Defective ECM Collagen type I secretion and organisation impairs cardiomyocyte homeostasis and maturation

4.4.4 Generation and validation of human HSP47 mutant cell lines 
5. Summary and Perspectives .....................................................................................117

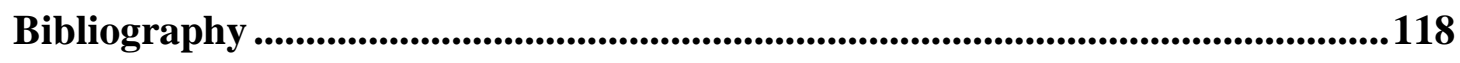

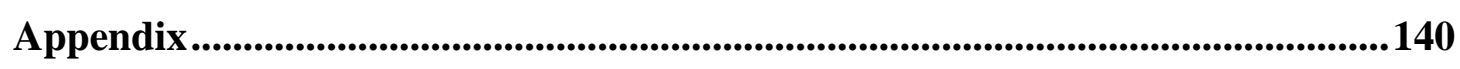

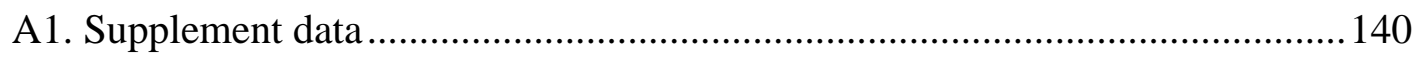

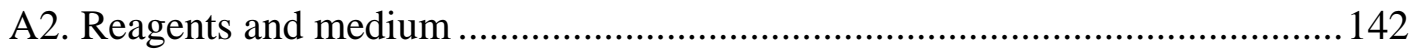




\section{$\underline{\text { Acknowledgements }}$}

I would like to thank all the people who have contributed in some way to the work described in my thesis. First and foremost, I would like to express my sincere gratitude to my supervisor Prof. Dr. Wolfram-Hubertus Zimmermann for allowing me the opportunity to come to Germany to undertake this project in his research group at the University of Göttingen, and enabling me the freedom to be creative and at the same time for his advice and direction throughout the duration of my studies.

I would furthermore like to thank both my thesis committee members Prof. Dr. Dörthe Katschinski and Prof. Dr. Peter Rehling for their continuous kindness, support and advice throughout my project and for their insightful comments and suggestions to widen my research scope from various perspectives.

My sincere thanks also go to Dr. Angelica Roa, Dr. James Hudson, and Dr. Peter Christalla. Without their precious support and knowledge, it would not be possible to conduct this research. Throughout the duration of my studies, each of them have taught me how to question thoughts, express ideas and develop as an independent scientist. Many thanks to Dr. Claudia Noack for establishing the HSP47 mutant hES2 cell lines, Dr. Sebastian Zeidler from his support with RNA-seq analysis, Prof. Kazuhiro Nagata (Kyoto Sangyo University, Japan) for kindly providing us the mouse HSP47 knockout cell line, and both Dr. Christof Lenz and Lisa Neuenroth from the Proteomic Core Facility UMG for their support with Mass Spectrometry.

I thank my fellow good friends and lab mates; Dr. Poh Loong Soong, Dr. Mei-Ling Chang Liao, Satish Galla and Elif Levent who have shared my ups and downs. Their support and care helped me overcome setbacks and stay focused on my studies. I greatly value their friendship and I deeply appreciate their belief in me.

I would like say a special thank you to my very caring and supportive girlfriend Naira Movsisyan, for her non-stop encouragement, kindness and support throughout the duration of my $\mathrm{PhD}$ studies. 
Finally, I would like to express my heart-felt gratitude to my family who I dedicate this thesis to. My parents, my sister and my brother-in-law have all been a constant source of love, inspiration, and strength throughout all these years. None of this would have been possible without the love, understanding and support of my wonderful family. 


\section{$\underline{\text { Abstract }}$}

The mammalian heart is a complex multicellular organ which undergoes many critical changes during development and under pathological conditions. Cardiac fibroblasts (cFBs) represent the predominant non-myocyte population in the adult heart and play essential roles in heart muscle development and homeostasis. The mechanisms how cFBs communicate with cardiomyocytes $(\mathrm{CMs})$ in contributing to the formation of a cardio-instructive environment remain unclear. In this study, we hypothesized that the mechanical and paracrine effects of $\mathrm{cFBs}$ and its secreted extracellular matrix (ECM) influence cardiac tissue formation and CM maturation. We utilized the 3D Engineered Heart Muscle (EHM) model of heart muscle development to investigate the role of cFBs in cardiac muscle formation in mouse and human systems. As collagen type I produced by cFBs comprises the main ECM protein in the heart, we furthermore investigated the influence of the collagen-specific molecular chaperone Heat Shock Protein 47 (HSP47) on CM maturation and assembly into functional syncytia, utilizing HSP47 knockout (KO) mouse FBs on 2D and 3D EHM culture platforms. By use of genetically engineered mouse and human embryonic stem cell (ESC) lines, we were firstly able to successfully generate by directed differentiation highly purified CMs to exclusively define the cellular constituents (CMs to non-myocytes) in EHMs. The generation of mature compact cardiac tissue with organotypic morphology and functionality was only feasible with the supplementation of FBs to EHMs. These observations can be explained by several identified mechanisms: FBs facilitate (1) cardiac tissue formation via integrin mediated signalling, (2) the secretion of a cardioinstructive ECM environment, important in mediating heart muscle maturation. Utilizing HSP47 KO FBs, we demonstrated the absence of HSP47 in FBs impairs both ECM-collagen deposition and CM morphology and functionality in both 2D and 3D EHM mouse culture systems. We were able to furthermore recently establish the generation of two mutant HSP47 human ESC lines via the CRISPR/Cas9 system. In conclusion, FBs play a prerequisite role in controlling and inducing cardiac tissue formation and maturation. Furthermore, we identified HSP47 to be an important regulator of cardiac tissue maturation. 


\section{$\underline{\text { List of Figures }}$}

Figure 1. Intercellular communication in the heart.

Figure 2. Roles of cardiac fibroblasts and their impact on different aspects of cardiac structure and function.

Figure 3. Schematic overview of the changes in ECM composition during different physiological states in heart.

Figure 4. Molecular structure of collagen type I.............................13

Figure 5. Schematic representation of processes involved in the folding and assembly of procollagen in the endoplasmic reticulum (ER) during collagen biogenesis.

Figure 6. Schematic representation of the possible function of HSP47 in the folding and assembly of procollagen in the ER

Figure 7. Representation of the integrin family

Figure 8. A simplified schematic of bi-directional ECM-cell signalling interfaces in the myocardium).

Figure 9. Design of casting molds for mouse and human EHM culture. 35

Figure 10. Schematic overview outlining the formation and assessment of EHMs...37

Figure 11. The measurement of soluble collagen secretion in MEFs 48

Figure 12. CM differentiation and purification of $\alpha \mathrm{MHC}-\mathrm{NeoR} \mathrm{mESCs}$. 61

Figure 13. CM differentiation and purification of hES2-CMs. 62

Figure 14. Advanced mCM maturation in cFB-supplemented EHMs.

Figure 15. Collagen type I binding integrins ITGA1, ITGA2 and ITGB1 are highly expressed in mouse and human FBs.

Figure 16. Higher abundance of collagen-binding Itgal, Itga2 and Itgb1 subunit transcripts in FB populations.

Figure 17. Integrin subunit transcript abundance in hES2-enriched CMs and FB populations.

Figure 18. Integrin-mediated signalling in FBs facilitate EHM formation. 70 
Figure 19. cFBs construct a heterogeneous ECM architecture in mouse EHMs......73

Figure 20. Collagen modifying and ECM-related transcripts are highly expressed in human FBs.

Figure 21. Collagen processing HSP47 is highly expressed in mouse FBs compared to $\mathrm{mCMs}$

Figure 22. Collagen processing HSP47 is highly expressed in human FBs compared to CMs.

Figure 23. Confirmation of Hsp47 gene targeted disruption in HSP47 KO MEFs. ...80

Figure 24. Absence of ER localized HSP47 at protein level in HSP47 KO MEFs. ... 82

Figure 25. Expression of ER-resident chaperone PDI in HSP47 KO MEFs. 83

Figure 26. Absence of HSP47 expression in MEFs reduces secretion of soluble collagen.

Figure 27. Reduced collagen type I protein incorporation into the ECM in HSP47 KO MEFs

Figure 28. Venn diagram illustrating the number of proteins identified in ECM fractions from WT- and HSP47 KO MEFs

Figure 29. Generation of denuded FB-derived ECM before and after decellularization.

Figure 30. Reduced Collagen type I fibril formation and deposition in HSP47 KO MEF-derived ECMs

Figure 31. Impaired CM morphology on collagen defective HSP47 KO MEF-derived ECMs. .91

Figure 32. Immature EHMs after supplementation with HSP47 KO MEFs .95

Figure 33. Reduced Itg $\beta 1$ integrin expression in HSP47 KO MEFs. 96

Figure 34. PCR genotyping of SERPINH1 homozygous mutant cell lines. .97

Figure 35. Confirmation of the HSP47 mutations in hES2 cells. .98

Figure S1. Advanced mCM maturation in cFB-supplemented EHMs. 140

Figure S2. Validation of Anti-Integrin alpha 1 antibody for immunoblotting analysis in human samples. 


\section{$\underline{\text { List of Tables }}$}

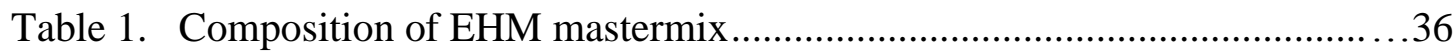

Table 2. List of primary and secondary antibodies/fluorescent conjugates used for protein biochemical analysis....................................41

Table 3. Thermocycler conditions used for PCR …............................................43

Table 4. Primers used in RT-PCR and qPCR analysis .........................................45

Table 5. Primers for the genotyping of the SERPINH1 mutation hES2 cells........57

Table 6. Thermocycler conditions used for genotype PCR ........................58 


\section{Abbreviations}

aa

AA

ACE

ACTN2

AEBP1

AGC

ANOVA

ANXA2

AR

ARB

bFGF

BCAM

BGN

bp

BSA

CALR

CAPG

Cas9

CASQ

$\mathrm{CBFHH}$

cDNA

$\mathrm{cFB}$

$\mathrm{CM}$

$\mathrm{CO}_{2}$

COL1A1

COL1A2

CRELD2

CRISPR

CRTAP

CSPG2
Amino acid

Ascorbic Acid

Angiotensin Converting Enzyme

Alpha-actinin 2

Adipocyte Enhancer-Binding Protein 1

Automatic Gain Control

Analysis of variance

Annexin 2

Aspect Ratio

Angiotensin Receptor Blockers

Basic Fibroblast Growth Factor

Basal Cell Adhesion Molecule

Biglycan

Base pair

Bovine Serum Albumin

Calreticulin

Capping Protein (Actin filament), Gelsolin-like

CRISPR associated protein 9

Calsequestrin

Calcium and Bicarbonate-free Hanks with HEPES

complimentary DNA

Cardiac Fibroblast

Cardiomyocyte

Carbon Dioxide

Collagen type I, alpha 1

Collagen type I, alpha 2

Cysteine-Rich with EGF-Like Domain protein 2

Clustered Regularly Interspaced Short

Palindromic Repeats

Cartilage Associated Protein

Chondroitin Sulfate Proteoglycan 2 
$\mathrm{Ct}$

CTNI

Cx

DAPI

DCN

DDR2

DEPC

DMEM

DMSO

DNA

DNase

dNTP

DOC

Dpc

Dpn

EB

ECM

ED

EDTA

EHM

EHT

EMT

EndMT

ENPL

EPDC

ER

ESC

$\mathrm{EtBr}$

$\mathrm{EtOH}$

F

FB

FBLN2

FBS

FC
Cycle threshold

Cardiac troponin I

Connexin

4', 6-diamidino-2-phenylindole

Decorin

Discoidin domain-containing receptor 2

Diethylpyrocarbonate

Dulbecco's Modified Eagle's medium

Dimethyl sulfoxide

Deoxyribonucleic acid

Deoxyribonuclease

Deoxyribonucleotide triphosphate

Deoxycholate

Days postcoitus

Days postnatal

Embryoid Bodies

Extracellular Matrix

Ehler-Danlos syndrome

Ethylenediaminetetraacetic acid

Engineered Heart Muscle

Engineered Heart Tissue

Epithelial-Mesenchymal Transition

Endothelial-Mesenchymal Transition

Endoplasmin

Epicardial-derived cells

Endoplasmic Reticulum

Embryonic stem cell

Ethidium Bromide

Ethanol

Forward primer

Fibroblast

Fibulin 2

Fetal Bovine Serum

Flow Cytometry 
FDR

FITC-A

FMOD

FN

FSC-A

FSP-1

FWHM

g

GAPDH

GAG

GO

GPC1

gRNA

GRP78

Gy

$\mathrm{H}_{2} \mathrm{O}$

HEPES

hES2

hFFB

HSP47

IBP7

$\mathrm{IgG}$

ILF2

IMDM

ITGA1

ITGA2

ITGB1

KEGG

KO

L

LAM

LIF

LUM

M
False Discovery Rate

Fluorescein isothiocyanate Area

Fibromodulin

Fibronectin

Forward Scatter Area

Fibroblast Specific Protein-1

Full Width at Half Maximum

Gravitational force

Glyceraldehyde-3-Phosphate Dehydrogenase

Glycosaminoglycan

Gene Ontology

Glypican 1

Guide RNA

Glucose-Regulated Protein 78

Gray

Water

4-(2-hydroxyethyl)-1-piperazineethanesulfonic acid

Human Embryonic Stem Cell line 2

Human foreskin fibroblasts

Heat Shock Protein 47

Insulin-like growth factor-Binding Protein 7

Immunoglobulin $\mathrm{G}$

Interleukin Enhancer Binding Factor 2

Iscove's modified Dulbecco's medium

Integrin, Alpha 1

Integrin, Alpha 2

Integrin, Beta 1

Kyoto Encyclopedia of Genes and Genomes

Knockout

Litre

Laminin subunit

Leukemia inhibitory factor

Lumican

Molar 


\begin{tabular}{|c|c|}
\hline MEF & Mouse Embryonic Fibroblast \\
\hline mESC & Mouse Embryonic Stem Cell \\
\hline$\alpha-\mathrm{MHC}$ & Myosin heavy chain, Alpha isoform \\
\hline$\beta-\mathrm{MHC}$ & Myosin heavy chain, Beta isoform \\
\hline mol & Mole \\
\hline MS & Mass Spectometry \\
\hline MYH6 & Myosin heavy chain, Alpha isoform \\
\hline MYH7 & Myosin heavy chain, Beta isoform \\
\hline $\mathrm{m} / \mathrm{z}$ & Mass-to-charge ratio \\
\hline $\mathrm{NaCl}$ & Sodium Chloride \\
\hline \multirow[t]{2}{*}{ NanoLC-MS/MS } & Nanoscale liquid chromatography coupled to tandem \\
\hline & mass spectrometry \\
\hline $\mathrm{NaOH}$ & Sodium Hydroxide \\
\hline NEAA & Non-Essential Amino Acids \\
\hline NeoR & Neomysin-Resistance \\
\hline $\mathrm{NH}_{4} \mathrm{OH}$ & Ammonium Hydroxide \\
\hline NHEJ & Non-homologous End Joining \\
\hline NID & Nidogen \\
\hline NMRI & Naval Medical Research Institute \\
\hline $\mathrm{OI}$ & Osteogenesis Imperfecta \\
\hline $\mathrm{P} 4 \mathrm{H}$ & Prolyl 4-Hydroxylase \\
\hline $\mathrm{P} 3 \mathrm{H}$ & Prolyl 3-Hydroxylase \\
\hline PAM & Protospacer Adjacent Motif \\
\hline PBS & Phosphate Buffered Saline \\
\hline PCOC1 & Procollagen C-Endopeptidase Enhancer 1 \\
\hline PCR & Polymerase Chain Reaction \\
\hline PDI & Protein Disulphide Isomerase \\
\hline PEDF & Pigment epithelium-derived factor \\
\hline PGS1 & Phosphatidylglycerophosphate Synthase 1 \\
\hline PLB & Phospholamban \\
\hline \multirow[t]{2}{*}{ PLOD } & Procollagen-Lysine, 2-Oxoglutarate 5- \\
\hline & Dioxygenase \\
\hline PPIase & Peptidyl-Prolyl cis-trans-Isomerase \\
\hline ProCol 1 & Procollagen type 1 \\
\hline
\end{tabular}




\begin{tabular}{|c|c|}
\hline $\mathrm{P} / \mathrm{S}$ & Penicillin Streptomycin \\
\hline PVDF & polyvinylidene Difluoride \\
\hline qPCR & Quantitative Polymerase Chain Reaction \\
\hline $\mathrm{R}$ & Reverse primer \\
\hline RIN & RNA Integrity Number \\
\hline RNA & Ribonucleic acid \\
\hline RNA-seq & RNA sequencing \\
\hline RPKM & $\begin{array}{l}\text { Reads Per Kilobase of transcript per Million } \\
\text { mapped reads }\end{array}$ \\
\hline RPMI & Roswell Park Memorial Institute \\
\hline RT & Room Temperature \\
\hline RYR2 & Ryanodine Receptor 2 \\
\hline SDC4 & Syndecan 4 \\
\hline SDS & Sodium Dodecyl Sulfate \\
\hline SDS-PAGE & Sodium Dodecyl Sulfate Polyacrylamide Gel \\
\hline & Electrophoresis \\
\hline SEM & Standard Error Mean \\
\hline SERCA2a & Sarcoplasmic reticulum $\mathrm{Ca}^{2+}$ ATPase pump \\
\hline Serpin & Serine Protease Inhibitors \\
\hline SERPINH1 & $\begin{array}{l}\text { Serpin Peptidase Inhibitor, Clade H, Member } 1 \\
\text { (Heat Shock Protein 47) }\end{array}$ \\
\hline SOTA & Self-Organizing Tree Algorithm \\
\hline Taq & Thermus aquaticus \\
\hline TBST & Tris-Buffered Saline and Tween 20 \\
\hline tdRFP & targeted Red Fluorescence Protein \\
\hline TGF- $\beta$ & Transforming Growth Factor beta \\
\hline $\mathrm{TNC}$ & Tenascin $\mathrm{C}$ \\
\hline TSP1 & Thrombospondin-1 \\
\hline $\mathrm{U}$ & Unit \\
\hline $\mathrm{V}$ & Volts \\
\hline WT & Wild type \\
\hline
\end{tabular}




\section{Introduction}

\subsection{Structure and cellular components of the heart}

The mammalian heart is a complex multicellular four chambered pumping organ which developmentally undergoes a series of crucial structural and functional changes leading to a mature and highly organized organ, capable of providing an optimal continuous blood flow to the rest of the body. The main cellular constituents of the heart include: cardiomyocytes (CMs) and non-myocytes (including; fibroblasts [FBs], endothelial cells and vascular smooth muscle cells), which are all surrounded by the extracellular matrix (ECM) environment (Figure 1). In addition, there are other transient cell types present in the heart which include: macrophages, lymphocytes and mast cells (Howard and Baudino, 2014). Our understanding of the developmental processes underlying the complexity of myocardial tissue and processes that maintain it have advanced over the recent years.

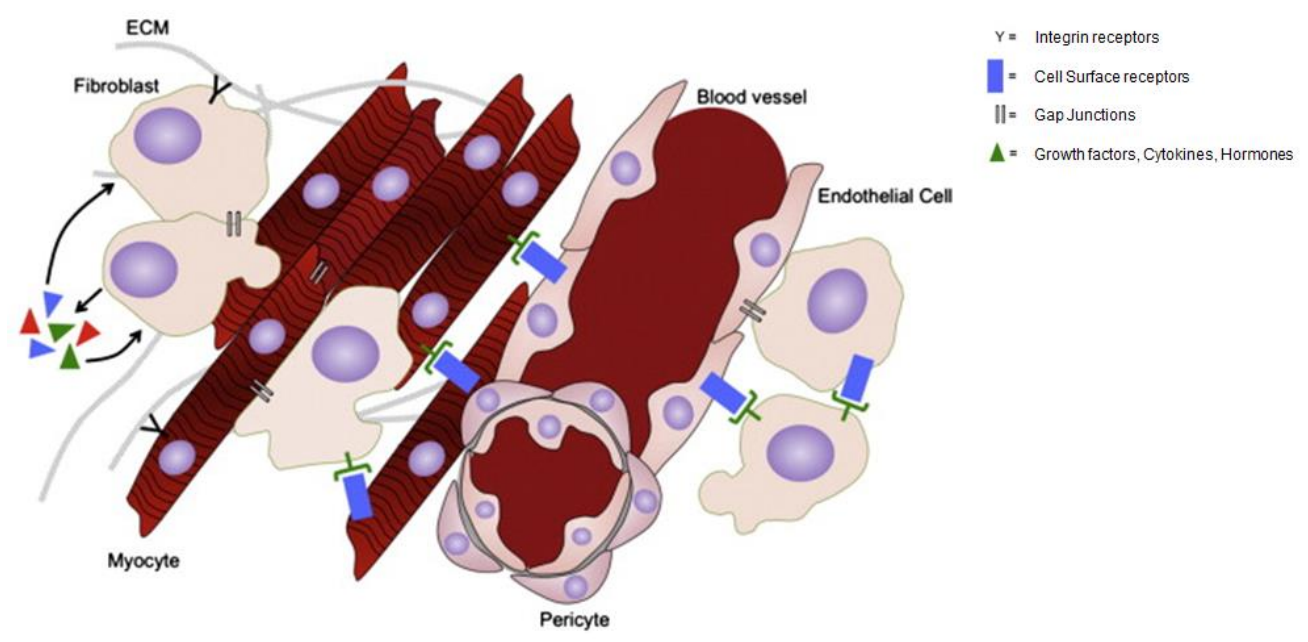

Figure 1. Intercellular communication in the heart. The complex interaction of cardiomyocytes, non-myocytes (including; fibroblast, smooth muscle cells, endothelial cells, macrophages and other cells of leukocytic orgin) and the surrounding extracellular matrix (ECM) are critical in maintaining homeostasis in the mammalian heart. Cells can interact through integrin receptors, cell surface molecules, gap junctions and through growth factors, cytokines and hormones, which can act in both an autocrine and paracrine manner (Taken from Howard and Baudino, 2014). 
CMs constitute approximately 20-30\% of cells in the human heart (Jugdutt, 2003), $50 \%$ in mouse heart (Banerjee et al., 2007) and 30\% in rat heart (Banerjee et al., 2007). The non-myocytes thus constitute the main cellular components of myocardial tissue (Pinto et al., 2016). These cells communicate with each other directly or indirectly via autocrine or paracrine signalling (Souders et al., 2009) and both the organization and the relative proportions of these cellular components play critical roles in heart development, physiology and pathology.

CMs are the key force-generating constituents of the myocardium and their maturation during development seems to be orchestrated in a temporal-specific manner from an embryonic elongated, ovoid shape, into a postnatal rod-shaped phenotype (Chacko, 1976). Each CM has a bundle of myofibrils divided into contractile units, or sarcomeres, which consist of a range of different contractile proteins including actin and myosin (Gregorio and Antin, 2000). The complex cellular components in the heart and their dynamic interactions are likely to collectively constitute a unique cardio-instructive environment, which can ultimately influence CM structure and function.

During the mammalian developmental stages of cardiac commitment and morphogenesis, CMs organize their structure by undergoing myofibrillogenesis (Du et al., 2008; Guan et al., 1999; Van der Loop et al., 1996). This results in the precise formation and arrangement of $\mathrm{CM}$ shape, assembly of the cardiac contractile apparatus and sarcomeric proteins, which is critical for normal cardiac growth and maturation. Disturbances of CM organization or contractile performance in the dynamic cardio-instructive environment during cardiac development usually have fatal consequences leading to cardiac malformations or prenatal lethality (Sussman et al., 1999). Moreover within the adult heart, rod-shaped CMs are anisotropically aligned in order to facilitate the propagation of electrical signals in directions parallel to the long axis of myocardial fibres (Kléber et al., 2011), which is necessary for efficient contraction, force transduction and electrical transmission in the heart to sustain effective cardiac output. At the cellular level, the mechanical and molecular signals which guide the orientation and alignment of myofibrils are not clearly understood, however, studies have identified that the formation of focal adhesions and 
cell-cell adhesions play a critical role in CM development (Petit and Thiery, 2000; Kovacic-Milivojevic et al., 2001).

The regulation of the heart requires the integration of various complex cellular mechanical, chemical and electrical signalling networks which are carefully orchestrated in order to sustain normal cardiac function and homeostasis. Alterations in any of these signalling networks can thus lead to disruption in other signals and ultimately result in cardiac dysfunction and disease (Howard and Baudino, 2014).

\subsection{Heart disease}

Heart disease is the leading cause of disability and mortality worldwide according to the World Health Organization (http://www.who.int/mediacentre/factsheets/fs317/en/) characterized by a loss or dysfunction of CMs (e.g., during and after a myocardial infarction). Heart failure is a progressive disease which has generally been characterized by the imbalance between cardiac output and the metabolic demands of the body. This usually results from reduced contractility of the heart (systolic dysfunction), insufficient filling of the heart (diastolic dysfunction) or more often a combination of both dysfunctions.

The mammalian heart is one of the least regenerative organs in the body and adult CMs have very little intrinsic capacity to regenerate following cardiac injury (Xin et al., 2013). Upon injury, the heart undergoes a series of initial morphological and function changes in order to compensate for $\mathrm{CM}$ loss at the aim to restore cardiac output. Over time however, chronic cardiac stress results in maladaptive responses, involving CM hypertrophy, cardiac fibrosis, ventricular dilation, chronic inflammation, and increased cellular apoptosis (Hill and Olsen 2008; Patton et al., 1998). Cardiac fibrosis is an integral component of most cardiac pathological conditions and is characterized by the excessive deposition of ECM proteins in the cardiac interstitium. Following acute myocardial infarction, the sudden loss of CMs triggers inflammation and thus ultimately leads to the replacement of dead myocardium with increased collagen-deposited fibrotic scars in the cardiac interstitial space (Frangogiannis, 2012), which is associated with increased mechanical stiffness 
and thus can contribute to both systolic and diastolic cardiac dysfunction (Janicki and Brower, 2002; Berk et al., 2007). These consequences above result in a vicious cycle towards pathological cardiac remodelling, further cardiac dysfunction and decompensated heart failure (Hill and Olsen, 2008; Patton et al., 1998).

Our understanding of cardiovascular disease has evolved over the last decade, leading to our current standard therapeutic interventions, which include $\beta$ - adrenergic receptor blockers, angiotensin-converting enzyme (ACE) inhibitors (or angiotensin receptor blockers, ARBs), aldosterone antagonists and/or diuretics to treat heart failure patients (Abraham et al., 2008; Bristow, 2000). However, despite improvements in the management of symptoms and overall mortality rates, these therapeutic interventions are only able to indirectly target heart failure and are not able to stimulate cardiomyocyte proliferation and regeneration, and can thus only delay heart failure progression with limited clinical benefits on preventing or reversing the onset of late stage heart failure (Packer et al., 1996; Lechat et al., 1998; Liu et al., 2010; Dhawan et al., 2007).

At present, the lack of ability to reverse the diseased cardiac phenotype is most likely due to our limited knowledge of the molecular mechanisms underlying cardiovascular disease pathologies. Most research has focused on malfunctions in CMs, disregarding the complex cellular composition of myocardial tissue. There has however been more of recent attention to the 'non-myocyte' cell populations in the heart, which compose the major cell population of it and are believed to play essential roles in CM development and function, hypertrophy (Brutsaert et al., 1996; Harada et al., 1997), and cardiac remodelling in heart diseases (Souders et al., 2009).

\subsection{Cardiac Tissue Engineering}

Cardiac tissue engineering based approaches focus on providing an artificial tissue mimic with natural organ structure and function. These aim to provide innovative in vitro research models for studying cardiac (patho) physiology, drug development and for use in therapeutic applications in order to restore the function of the damaged myocardium (van Spreeuwel et al., 2014; Christalla et al., 2012). The progress in this 
field however critically depends on the use of stem cell technologies and a greater understanding of biological processes which can govern these cells towards a desired phenotype, which may support the improvement of cardiac regeneration therapies and further facilitate the discovery of new possible targets to treat heart disease. Understanding the processes in driving CMs towards maturation in an organotypic manner or towards a pathological phenotype will be a pre-requisite to ultimately provide realistic cardiac tissue models (Christalla et al., 2012).

The process of creating functional three dimensional cardiac tissue constructs relies on the combination of cardiac cells with scaffolds or devices that facilitate cell growth, organization and differentiation (Griffith and Swartz, 2006). Despite our increasing understanding of developmental processes governing cardiac development, there is still a lack of understanding concerning the complex interplay of cellular and extracellular factors, which are most likely to constitute a unique cardio-instructive environment and control cardiogenesis and maturation of the myocardium (Christalla et al., 2012).

\subsubsection{Engineered heart muscle (EHM) to study cell interactions}

Tissue models reconstituted from specified cell types and ECM components provide simplified 3D biological systems to study cell-ECM interactions in several different biological processes, including tissue homeostasis and development (Bell et al., 1979; Grinnell, 1994). Several groups including our own have previously demonstrated that CMs from neonatal rats and embryonic chicken can be reconstituted to 3D cardiac tissue-like constructs (Eschenhagen et al., 1997; Akins et al., 1999; Bursac et al., 1999; Carrier et al., 1999; Li et al., 1999; Leor et al., 2000; Li et al., 2000; Zimmermann et al., 2000; Kofidis et al., 2002; Krupnick et al., 2002; Shimizu et al., 2002). Studies in our lab use a novel simplified in vitro 3D model of heart muscle development termed 'Engineered Heart Muscle' (EHM). EHMs are collagen-hydrogel based constructs comprising of the most abundant cellular and ECM components of native heart muscle, which resemble morphological and functional properties of the native myocardium (Tiburcy et al., 2011; Zimmermann et al., 2002) and can be used to address the importance of cell-ECM interactions in the myocardium. The 
development of our EHM model was originally influenced by a method developed to measure the functional and mechanical properties of tissue constructs assembled with chicken embryonic FBs (Kolodney and Elson, 1993).

Several lines of evidence suggest that non-myocytes both support the growth of CMs in culture, and are essential for the generation of cardiac tissue constructs. Our group has previously demonstrated the importance of the non-myocyte cell fraction in the heart for being essential in maintaining a cardiogenic environment in EHMs (Naito et al., 2006). EHMs comprised of mixed heart cell populations (CMs and non-myocytes) showed advanced tissue structure and displayed increased contractile and passive forces, when compared to EHMs comprised of an enriched CM cell population (Naito et al., 2006). Furthermore, Vunjak-Novakovic and colleagues demonstrated that poly (glycerol sebacate) constructs seeded with both cardiac FBs and CMs developed much better tissue structure and function compared to those cultured with pure CMs (Radisic et al., 2008). In addition, several other studies using other bioartificial cardiac tissue-based models have demonstrated the importance of FBs for CM survival and coupling, and the formation of well structured, functional myocardial tissue (Kensah et al., 2012; Liau et al., 2011; Pfannkuche et al., 2010). Finally, the addition of endothelial cells and stromal cells to human embryonic stem cell-derived CMs has been demonstrated to improve both cardiac tissue structure and function (Lesman et al., 2010; Stevens et al., 2009; Tulloch et al., 2011). The mechanisms underlying how FBs or other non-myocytes are able to promote $\mathrm{CM}$ maturation in these models of heart muscle development are however not clearly understood.

It is becoming increasingly recognised that cardiac fibroblasts (cFBs) are the most abundant cell type in the myocardium and contribute importantly to multiple aspects of myocardial function and pathophysiology (Souders et al., 2009). FBs are able to interact with CMs via paracrine and mechanical signalling mechanisms. The utilization of this EHM model to study the functional and mechanistic roles of cFBs may thus provide further insight to how these cells may control cardiogenesis and CM maturation in heart muscle development. 


\subsection{Cardiac fibroblasts in the heart}

FBs are found in all vertebrate organs and are usually defined as cells of mesenchymal origin (Souders et al., 2009). cFBs are key components of the myocardium and have been found to play critical roles in cardiac development, maintaining normal cardiac function, as well as cardiac remodelling during pathological conditions, such as myocardial infarction and hypertension (Souders et al., 2009).

Morphologically, these cells are flat and spindle-shaped in culture and lack a basement membrane (Souders et al., 2009). Although a lot of research focus has paid attention to FBs, there is no truly definitive cell-specific marker to characterize these cells. Three proteins primarily used in studying FBs are the collagen receptor Discoidin Domain Receptor 2 (DDR2) and Vimentin, present in the fibroblasts intermediate filaments, and Fibroblast Specific Protein 1 (FSP-1; also known as S100A4), a filament associated calcium-binding protein in fibroblasts (Zeisberg and Kalluri, 2010; Fan et al., 2012). These proteins however lack fibroblast specificity (Vimentin and DDR2) or are only specific for a subset of cFB populations (FSP-1). There is thus emerging evidence that cFBs are not a homologous cell type, but highly heterogenous cell populations existing in the myocardium (Krenning et al., 2010; Zeisberg et al., 2007).

\subsubsection{Origins of cardiac fibroblasts}

During cardiac development, epicardium-derived cells over the embryonic heart undergo epithelial-mesenchymal transformation (EMT) and consequently differentiate into fibroblasts (Lie-Venema et al., 2007). EMT has been shown to be induced primarily by periostin (Norris et al., 2008) and Transforming Growth Factor $\beta$ (TGFß) (Olivey et al., 2006). In addition, FBs have also been shown to derive from endothelial cells from the endocardium via endothelial mesenchymal transformation (EndMT) (deLange et al., 2004). Both epicardium- and endothelial- derived cells however become quiescent after embryonic development. Following myocardial injury, hematopoetic cells derived from the bone marrow can be recruited to the site 
of injury and transform to cFBs or myofibroblasts (Fan et al., 2012). Myofibroblasts play critical roles in remodelling processes in cardiac pathology (Calderone et al., 2006; Brown et al., 2005).

\subsubsection{Function of cardiac fibroblasts in the heart}

cFBs are involved in numerous functions in the heart including primarily the secretion and remodelling of the ECM. In addition, cell-to-cell communication with neighbouring CMs, endothelial cells, other FBs and smooth muscle cells, can impact cellular events such as: angiogenesis, CM hypertrophy, cell proliferation, and apoptosis (Fan et al., 2012). FBs are able to exert mechanical force on CMs by contracting the collagen network that forms the ECM (Howard and Baudino, 2014). FBs also produce various growth factors and cytokines (Souders et al., 2009). It is becoming more appreciated that FBs furthermore support the electrical activity of the heart, as they are conductors with high membrane resistance (Kohl, 2003) and are responsible in electrically separating the atria and ventricle by forming an insulating layer to facilitate in proper sequential contraction of the heart (Liu et al., 2008). cFBs are coupled to $\mathrm{CMs}$ via gap junctions through connexins ( $\mathrm{Cx} 40, \mathrm{Cx} 43$ and $\mathrm{Cx} 45)$ which are necessary in maintaining an optimal electrical conduction in the heart (Camelliti et al., 2004a; Gaudesius et al., 2003). Many studies have termed these cells as 'sentinel', as they can both sense and respond to changes in mechanical, chemical and electrical signals in the heart (Souders et al., 2009; Baudino et al., 2006) (Figure 2). 


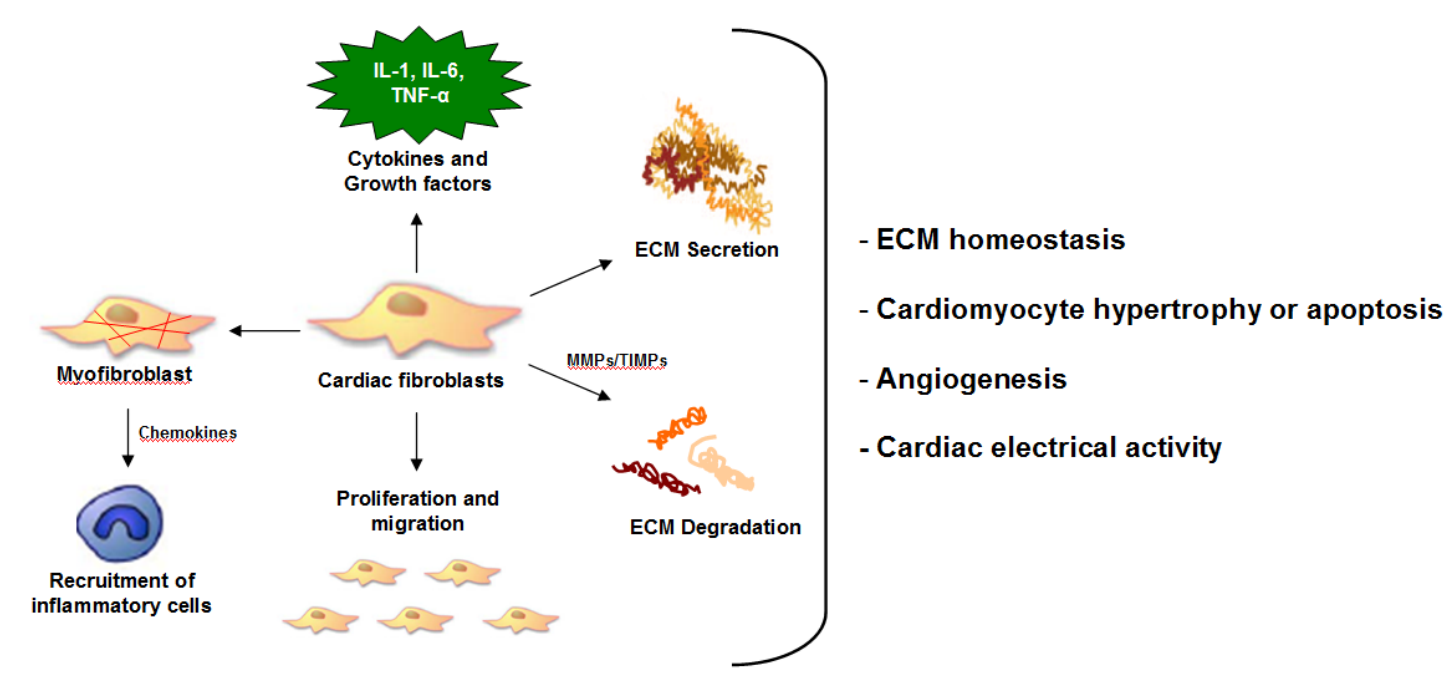

Figure 2. Roles of cardiac fibroblasts and their impact on different aspects of cardiac structure and function (Adapted from Souders et al., 2009; Fan et al., 2012).

It is thus becoming clearer that cFBs play a key role for constructing a cardiogenic environment and contribute to structural, biomechanical, biochemical and electrical properties of the heart, thus maintaining appropriate form and function of the myocardium (Chilton et al., 2007; Kohl, 2003; Louault et al., 2008; Camelliti et al., 2004b). Since most cardiac diseases are associated with an increase of fibrosis in the heart (Boudoulas and Hatzopoulos, 2009), it is clear that FBs and its derived ECM play significant roles during cardiac pathological remodelling.

\subsection{Cardiac fibroblasts and the ECM}

cFBs are the key cell type in the heart responsible for the deposition and maintenance of ECM homeostasis during cardiac development, normal physiology, and during remodelling in heart disease (Fan et al., 2012). The ECM is a complex, heterogeneous network of soluble and insoluble protein, which provides a structural framework and support for CMs and other cell types in the myocardium. This mechanical framework permits cell-to-cell interactions for healthy tissue formation and maintenance (Bowers et al., 2010). The cardiac ECM under normal conditions provides an anisotropic structural scaffold to guide CM alignment and has been shown to be essential in supporting the efficient contraction and relaxation of CMs (Li et al., 2014), and thus 
proper myocardial structural integrity and pump function (Curtis and Russel, 2011). The ECM scaffold enables optimal force transduction for mechanical work, electric transmission and chemical signals within the myocardial environment during development and homeostasis, as well as in response to physiological stress or injury (Li et al., 2014; Bowers et al., 2010).

The major component of the ECM is primarily collagen, and to a lesser extent a wide array other different proteins including; fibronectin, laminin fibrillin, elastin, proteoglycans and glycosaminoglycans (GAGs). Both collagen and elastin have been considered as the 'bricks' of the ECM due to their contribution to its mechanical properties, and GAGs are thought to contribute to the 'gel-like' characteristics of the ECM (Fernandes et al., 2009). The ECM is very dynamic as numerous studies have revealed that both the structural organization and composition of the ECM differs during distinct physiological states. For instance, during cardiac development and disease, a number of growth factors such as platelet-derived growth factor (PDGF) and TGF- $\beta$ can induce the activation of a wide array of different ECM proteins in cFBs (Butt et al., 1995). In addition to the synthesis of ECM proteins, cFBs also produce a variety of different ECM-degrading proteins including; matrix metalloproteinase (MMPs) and tissue inhibitors of matrix metalloproteinase (TIMPs), which are all critical in maintaining ECM homeostasis and can impact cardiac function. Furthermore, the ECM contains a number of growth factors and cytokines than can also impact CM function (Fan et al., 2012).

\subsubsection{ECM remodelling in the developing and pathological heart}

The different ECM microenvironments present during cardiac development, homeostasis and pathological conditions display unique biochemical niches. These different microenvironments can thus influence cardiac form and function, which are primarily driven by cell-cell and cell-ECM interactions (Bowers et al., 2010). Numerous studies have collectively identified differences in specific ECM components during different physiological states of the heart (Figure 3). 


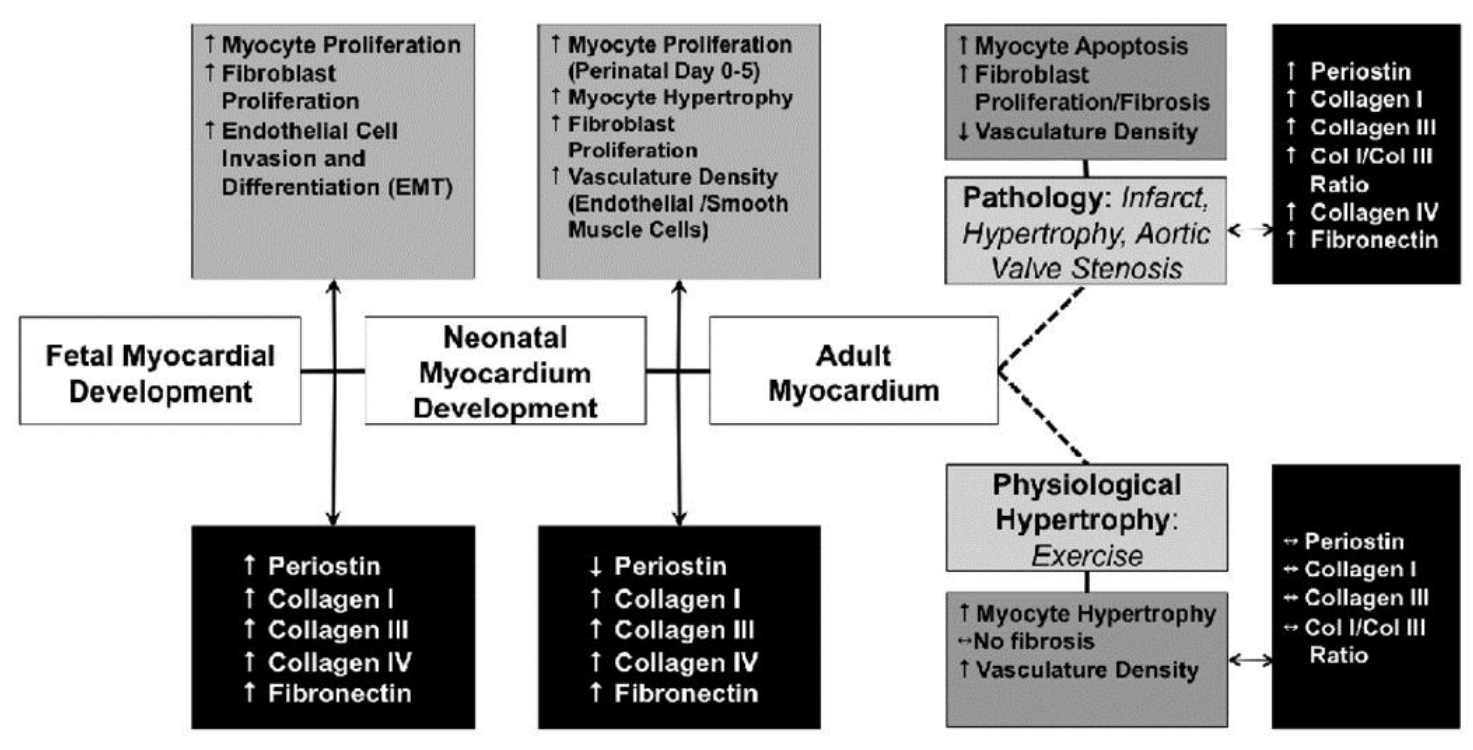

Figure 3. Schematic overview of the changes in ECM composition during different physiological states in heart. The changes in cellular (grey boxes) and ECM components (black boxes) during different cardiac physiological states (white boxes) (Taken from Bowers et al., 2010).

The critical roles of ECM in tissue formation and homeostasis have been demonstrated in a number mouse models for certain ECM proteins as well as some human disorders (Aszódi et al., 2006). Diseases such as Osteogenesis Imperfecta (OI), Ehler-Danlos (ED) syndrome and epidermolysis bullosa caused by mutation in genes encoding for structural proteins in collagens, can result in fatal outcomes (Fernandes et al., 2009). Within the context of heart diseases, the ECM initially undergoes dynamic changes in order to restore function of the myocardium. Over time, sustained ECM deposition (primarily collagens) results in a stiffer myocardium, which impairs mechanical function and electrical dynamics of the heart ( $\mathrm{Li}$ et al., 2014; Bowers et al., 2010).

The knowledge of the role of the ECM during cardiac development is however still limited and it is not clearly understood which ECM factors are on the one hand; supporting CM growth, maturation and physiological hypertrophy, and on the other hand contribute to disease development and progression. 


\subsection{Collagens in the heart}

The most abundant proteins in the ECM are members of the collagen family, which are centrally involved in the formation of fibrillar networks of the ECM, basement membranes, as well as structures of the ECM (Gelse et al., 2003). More than 28 different collagen types in various vertebrate tissues have been identified. These can be divided into two major classes; the fibrillary and non-fibrillar collagens (Jugdutt, 2003). Among the collagen family, five collagen types (I, II, III, V and XI) form fibrils which provide the structural framework of tissues (Jugdutt, 2003).

Fibrillar collagen types I and III are the most abundant components in the cardiac ECM (De Jong et al., 2012; Fan et al., 2012) with collagen type I making up 85\% and collagen type III $11 \%$ of total collagen in the myocardium (DeSouza, 2002; Jugdutt, 2003). Collagen fibres in the myocardium are able to form collagen networks that provide cardiac strength during contractile forces (Jugdutt, 2003). In addition, these collagen fibres play a role in connecting neighbouring CMs (Eghbali and Weber, 1990). In the context of heart diseases, the myocardium is characterized by excessive collagen type I deposition, collagen fibril disruption, structural ECM remodelling and loss of CMs via apoptosis and necrosis (Swynghedauw, 1999).

During cardiac development, fibrillar collagen I is initially expressed throughout the developing leaflets of the atrioventricular valves, but this gradually becomes restricted to the ventricular sides of the valve leaflets after birth (Kruithof et al., 2007; Lincoln et al., 2004). Collagen I is additionally expressed in the aortic walls. Studies have shown the disruption of the Col1A1 gene in mice, are more susceptible to aortic dissection and rupture. Furthermore, Mov13 mice possessing an insert mutation in the Col1A1 gene are embryonic lethal at 12-14 days postcoitus (dpc), due to rupturing of developing blood vessels (Lohler et al., 1984; Rahkonen et al., 2004).

Many more animal studies have revealed that different collagen types are important during cardiac development (Metsaranta et al., 1992; Lincoln et al., 2004; Hinton et al., 2006; Liu et al., 1997; Polschl et al., 2004; Kruithof et al., 2007; Favor et al., 2006; Wenstrup et al., 2004; Bonaldo et al., 1998; Klewer et al., 1998), however 
currently little work has been performed to elucidate the roles of collagens in early stages of cardiac development.

\subsubsection{Structure and biosynthesis of collagen}

Knowledge into the molecular structure, biosynthesis, assembly and turnover of collagens is essential in order to understand both the developmental processes, as well as the pathological processes linked with cardiac disorders and many other human diseases (Gelse et al., 2003).

All collagens are right-handed triple helix structures which are composed of three $\alpha$ chains (Figure 4). Each $\alpha$-chain contains domains with repetitions of proline-rich amino-acid tripeptides $(\mathrm{Gly}-\mathrm{X}-\mathrm{Y})_{\mathrm{n}}$, in which $\mathrm{X}$ and $\mathrm{Y}$ are usually proline and hydroxyproline residues respectively, and flanked by $\mathrm{N}$ - and $\mathrm{C}$ - propeptide globular domains. These triple-helical structures can be either homotrimeric or heterotrimeric, depending on the collagen type (Canty et al., 2005; Gelse et al., 2003).

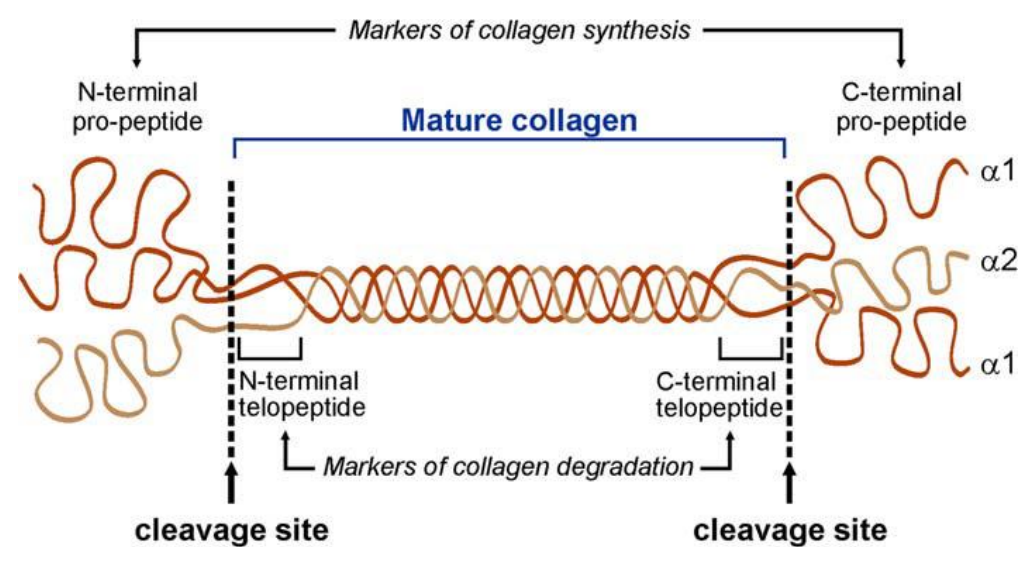

Figure 4. Molecular structure of collagen type I. Procollagen consists of two $\alpha 1-$ and $\alpha 2$ - chains which are wounded into a triple helix. Once procollagen is processed in the ER, propeptide domains at the $\mathrm{N}$ - and $\mathrm{C}$ terminals are cleaved, resulting in the formation of mature collagen. When collagen is degraded, telopeptides (from the $\mathrm{N}$ - and $\mathrm{C}$ - terminals) are cleaved and released (Taken from Fan et al., 2012).

Furthermore, depending on the collagen type, the proline- and lysine specific residues can be modified by post-translational modifications by enzymatic hydroxylation (Gelse et al., 2003). The Gly-X-Y helical repeats are the major motif present in fibrillar forming collagen types (I, III and V), which can result in triple helical structures of $300 \mathrm{~nm}$ in length (Von der Mark, 1999). Fibrillar collagen type I is the 
most abundant collagen in the body and the most extensively characterized (Gelse et al., 2003). The mechanisms involved in the biogenesis of collagen type I are described in Figure 5.

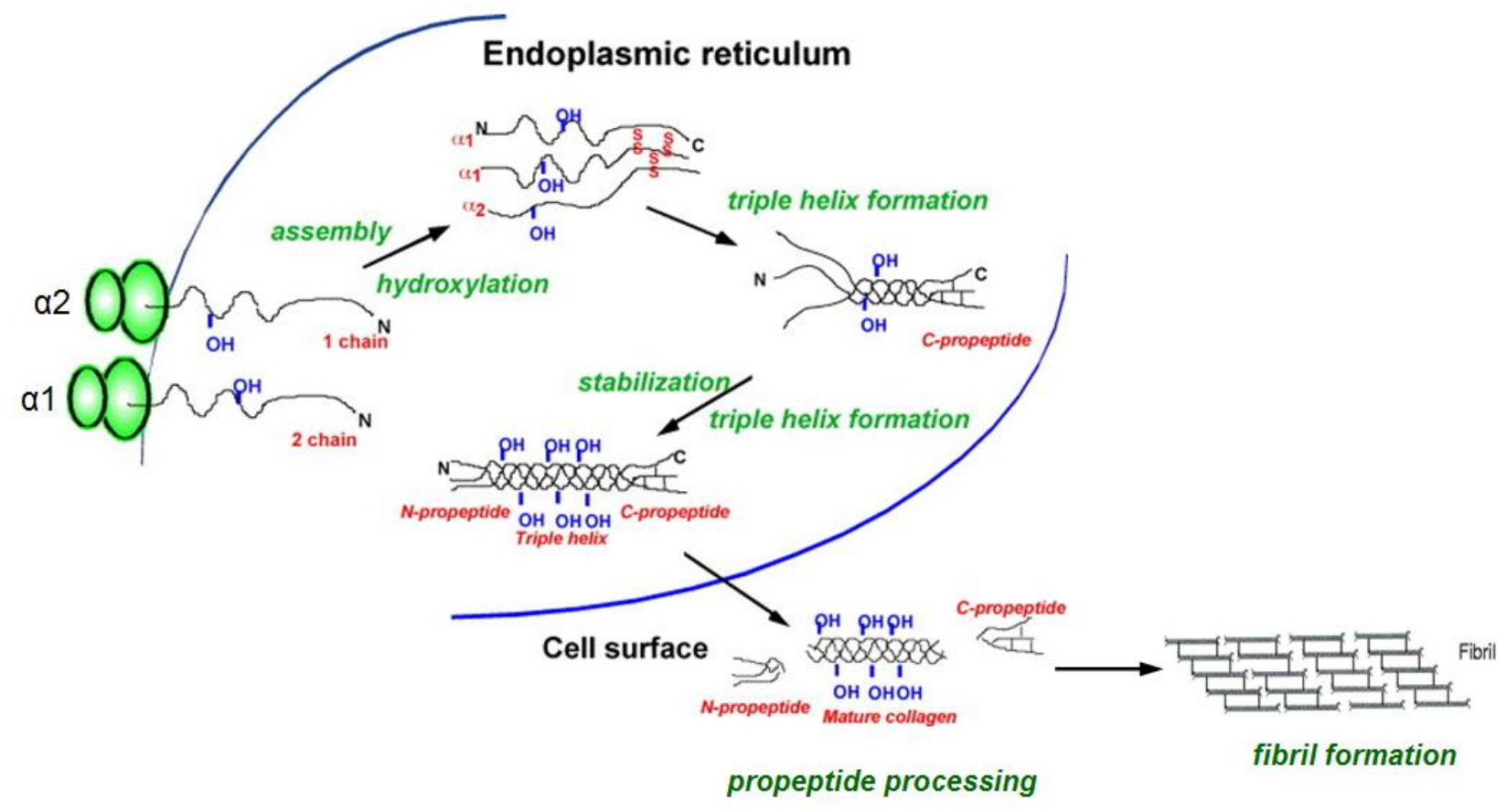

Figure 5. Schematic representation of processes involved in the folding and assembly of procollagen in the endoplasmic reticulum (ER) during collagen biogenesis. Newly synthesized collagen $\alpha 1$ - and $\alpha 2$ - chains are inserted into the ER and undergo hydroxylation and post-translational modifications. All $3 \alpha$-chains assemble at the C-propeptide region, are covalently linked by disulphide bonds, and triple helix formation then proceeds from the $\mathrm{C}$ - to $\mathrm{N}$-terminus with the aid of several folding enzymes and molecular charperones. Triple helix procollagen is then transported out the ER and secreted out into the extracellular space. $\mathrm{N}$ - and C-propeptides are then cleaved off by $\mathrm{N}$ - and $\mathrm{C}$ propeptides to form mature collagen. Mature collagen is then assembled laterally with other neighbouring molecules to form collagen fibrils (Adapted from Nagata, 2003).

This initially starts with transcription of collagen genes within the nucleus of FBs and/or other collagen-producing cells. The regulation of the transcriptional processes involved in collagens depends largely on the cell type, but activities may also be controlled by various growth factors and cytokines (Von der Mark, 1981). The collagen type I triple helix is heterotrimeric and comprises of two identical $\alpha 1$ (I) chains and one $\alpha 2$ (I) chain, which are synthesized as large precursor pro- $\alpha$-chains. Subsequently, these individual $\alpha$-chains are co-translationally transported into the 
lumen of the endoplasmic reticulum (ER), where they undergo multiple steps of posttranslation modifications by a large number of molecular chaperones and enzymes to assist folding and trimerization (Gelse et al., 2003; Canty et al., 2005) (Figure 5).

Hydroxylation of specific proline (particularly at the $\mathrm{Y}$ position of the Gly-X-Y triplet) and lysine residues of the triple-helical domains are catalyzed by prolyl 3hydroxylase $(\mathrm{P} 3 \mathrm{H})$, prolyl 4-hydroxylase $(\mathrm{P} 4 \mathrm{H})$ and lysyl hydroxylase, during cotranslational transport into the ER (Gelse et al., 2003). All the three enzymes require the provision of ferrous ions, ascorbate, 2-oxogluterate and molecular oxygen as cofactors (Gelse et al., 2003). In procollagen, these hydroxyl groups are thought to form interchain hydrogen bonds that help stabilize the triple helix. Conditions preventing proline hydroxylation, such as the deficiency of ascorbic acid (vitamin C), have severe consequences. In scurvy, the disease caused by a dietary deficiency of vitamin $\mathrm{C}$, the defective pro- $\alpha$ chains that are synthesized fail to form stable triple helices and are thus immediately degraded within the cell (Alberts et al., 2002).

After hydroxylation, these 3 pro- $\alpha$-chains assemble at their C-propeptide regions. The globular structures of the $\mathrm{C}$-propeptide regions present in all 3 pro- $\alpha$-chains are then covalently linked by inter-chain disulphide bonds (Doege and Fessler, 1986) by protein disulphide isomerase (PDI) (Koivu et al., 1987) and triplex helix formation then proceeds from the $\mathrm{C}$-terminus to the $\mathrm{N}$-terminus in a zipper-like manner. In the procollagen molecule, glycine residues located in the inner-most region of the triplehelix and the hydroxylated proline residues (at the $\mathrm{Y}$ position) contribute to the stabilization of the triple helix (Nagata, 2003).

A number of folding enzymes and molecular chaperones including; glucose-regulated protein 78 (GRP78; or BiP), GRP94, peptidyl-prolyl cis-trans-isomerase (PPIase) (Lang et al., 1987) and the collagen-specific chaperone Heat Shock Protein 47 (HSP47) (Clarke et al., 1991) have been shown to associate with newly synthesized procollagen in the ER and assist in facilitating efficient formation and correct folding of procollagen chains (Nagata, 2003; Gelse et al., 2003; Canty et al., 2005). HSP47 has been shown to be critical for collagen molecular maturation in the ER (as further discussed in Section 1.6.2). After processing and procollagen assembly, only the triple-helical form of procollagen is transported from the ER in the Golgi apparatus, 
where these procollagen trimers are packaged into secretory vesicles and transported out of cells into the extracellular space (Gelse et al., 2003; Nagata, 2003). Following secretion, the $\mathrm{N}$ - and C-propeptides are cleaved off by $\mathrm{N}$ - and $\mathrm{C}$ - propeptidases, respectively, to form mature collagen. The resulting triple-helical collagen molecules assemble laterally and intermolecular covalent cross-links occur within and between adjacent structures to form a stable fibrillar network, which contributes to the mechanical stiffness of collagen fibrils.

\subsubsection{HSP47 in the biogenesis of collagen}

Heat shock protein 47 (HSP47) is a $47 \mathrm{KDa}$ collagen-specific glycoprotein molecular chaperone residing in the ER of collagen-producing cells and has been shown to be essential for embryonic development and maturation of collagen types I and IV (Ishida and Nagata, 2011). This protein is a member of the serpin (serine protease inhibitors) superfamily and possesses a unique characteristic as it specifically binds to procollagens in the ER. HSP47 exclusively binds to collagen, recognizing an arginine at the Y position of a procollagen Gly-X-Y helical repeat (Widmer et al., 2012). In contrast to many other ER chaperones involved in collagen processing and maturation, HSP47 preferentially recognizes the folded triple-helical conformation of its client (Widner et al., 2012).

Furthermore, HSP47 expression patterns in different cells and tissues always correlate closely with collagen expression (Nagata and Hosokawa, 1996; Nagata, 1998). HSP47 synthesis has not been observed in cells which do not synthesize collagen (Nagata and Hosokawa, 1996). Furthermore, evaluated expressions levels of HSP47 have also been observed in several fibrosis models (Nagata et al., 1986; Taguchi and Razzaque, 2007; Takechi et al., 1992). Several roles have been suggested for HSP47 in collagen biogenesis. These include assisting the correct folding and stabilization of triplehelical procollagen (Tasab et al., 2000; Nagata et al., 1996), and the control and prevention of unfolded procollagen aggregates in the ER. HSP47 has been shown to be associated with triple-helical procollagen rather than unfolded $\alpha$-procollagen chains (Tasab et al., 2002; Koide et al., 2006; Thompson et al., 2003), and also assists during its export into the cis-Golgi, where it then dissociates from procollagen in a 
pH-dependant manner and is recycled back to the ER (Lamande and Bateman 1999; Satoh et al., 1996).

The importance of HSP47 in embryonic development and collagen maturation has been demonstrated previously from gene ablation studies from the lab of Kazuhiro Nagata. Studies showing the disruption of murine HSP47 using homologous recombination caused embryonic lethality in mice at $11.5 \mathrm{dpc}$. These mouse embryos displayed impaired defects in the formation of collagen fibres and basement membrane (Nagai et al., 2000), caused by aberrant procollagen folding in the ER (Figure 6). At $10.5 \mathrm{dpc}$, the embryos from HSP47 knockout (KO) mice showed developmental retardation and cardiac hypertrophy (Nagai et al., 2000; Ishida and Nagata, 2011).

(A)

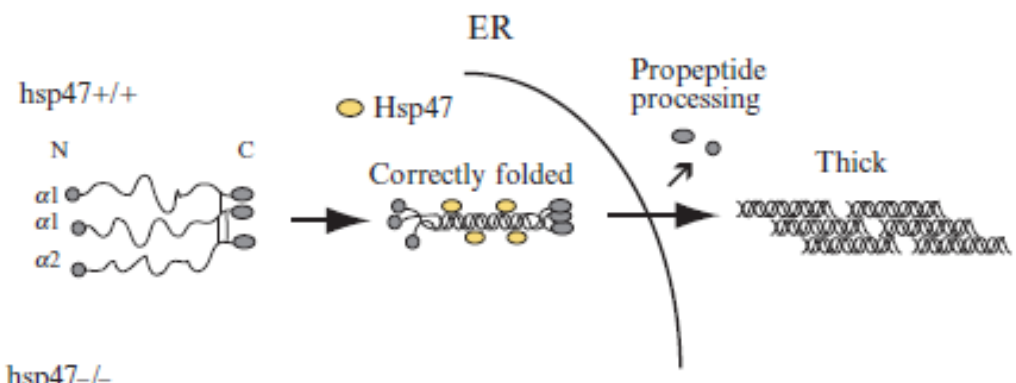

(B) hsp47-/-

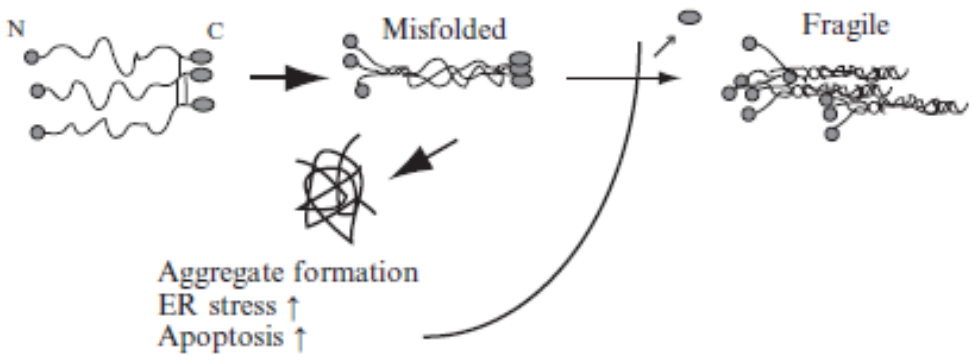

Figure 6. Schematic representation of the possible function of HSP47 in the folding and assembly of procollagen in the ER. Collagen forms trimers in the ER and triple helix formation proceeds from the $\mathrm{C}$ - to $\mathrm{N}$ - terminus in a zipper-like manner. (A) In the presence of HSP47, correctly folded collagen is exported out the ER and secreted into the extracellular space, and rigid collagen fibrils are formed by assistance of HSP47. (B) In the absence of HSP47 (in HSP47 ${ }^{-1-}$ [KO] fibroblasts), collagen folding is impaired and is retained in the ER. Aggregate formation and the induction of ER stress are observed, finally triggering ER stress induced apoptosis. Note that N-propeptides of procollagen secreted from HSP47 KO fibroblasts are not cleaved/processed due to improper folding of the procollagen triple helix, which results in abnormal collagen fibril formation (Adapted from Ishida and Nagata, 2011). 
Furthermore, collagen type I secreted from HSP47 KO FBs was markedly decreased and displayed abnormal and misaligned fibrillar structures with thin and branched fibrils (Ishida et al., 2006). Triple helix formation, secretion, and processing of the $\mathrm{N}$ terminal propeptide of procollagen type 1 was impaired, which thus resulted in the failure to accumulate collagen fibres into the ECM. The secretion of collagen type I and IV (Matsuoka et al., 2004) was delayed leading to the intracellular accumulation of procollagen in the ER. The secretion and deposition of other ECM proteins including fibronectin and laminin were however shown to be unaffected (Ishida et al., 2006), indicating that HSP47 disruption specifically altered procollagen maturation.

Since HSP47 is essential for mouse development, mutations in human HSP47 would thus be expected to be lethal. The ECM is required for both bone and tissue formation, and additional studies have revealed that point mutations in the SERPINH1 gene (encoding for human HSP47) are present in one of the subtypes of the recessive Osteogenesis Imperfecta (OI) bone disorder in humans (Christiansen et al., 2010) and dog, i.e. in Dachshunds (Drogemuller et al., 2009; Lindert et al., 2015). These results further highlight the importance of HSP47 as a collagen-specific molecular chaperone which is necessary for the formation of procollagen triple helices in the ER.

HSP47 is closely related with collagen-related diseases, including fibrosis in various organs. It has been shown to be over expressed in various human and experimental fibrotic diseases which include; glomeruloscerosis pulmonary fibrosis, liver cirrosis and fibrosis following myocardium infarction (Sauk et al., 2005). Furthermore, studies have shown that suppression of HSP47 expression can reduce the accumulation of collagens in order to delay the progression of fibrotic diseases in experimental animal models (Taguchi and Razzaque, 2007). HSP47 thus provides a selective target to manipulate collagen production in the ECM, which may thus have enormous clinical impact in controlling a wide range of fibrotic diseases.

Although HSP47 is essential for embryonic development and collagen maturation, it is not known with respect to the heart whether its influence in collagen processing and maturation can impact the functionality and maturation of CM during development, normal homeostasis or during myocardial fibrosis. 


\subsection{Cell-ECM communication via integrin receptors}

Increasing evidence suggests that mechanical interactions between the ECM and CMs, as well as physical interactions transmitted between other neighbouring cells play important roles in regulating signalling pathways, which can thus influence the form and function of the myocardium during development (Sheehy et al., 2012). Cells are able to sense external mechanical cues primarily through interactions with the ECM via integrin binding and from neighbouring cells through intracellular junctions (Chen et al., 2004).

Integrins are a diverse family of heterodimeric, transmembrane receptors composed of $18 \alpha$ and $8 \beta$ heterodimeric subunits (Hynes, 1987; Giancotti and Ruoslahti, 1999) that mediate the attachment of cells to the ECM, as well as in mediating cell-cell interactions (Barczyk et al., 2010). In total, there are 24 integrin heterodimeric receptors (formed from one $\alpha$ and one $\beta$ subunit) which can specifically bind to a wide range of different ECM molecules/ligands with overlapping binding affinities (Alam et al., 2007). Additional complexity is further presented by the numerous splice variant isoforms of individual $\alpha$ and $\beta$ subunits, including some expressed in the heart (de Melker and Sonnenberg, 1999; Burkin and Kaufman, 1999) (Figure 7).

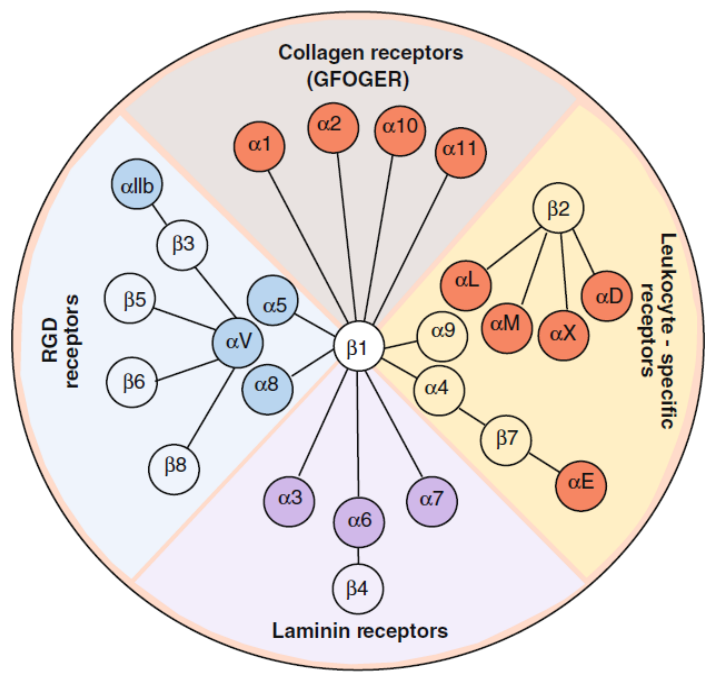

Figure 7. Representation of the integrin family. This includes the classification of integrin receptors based on their ligand binding ability. Four different categories have been presented; 1) Collagen binding, 2) RGD binding, 3) Laminin binding and 4) Leukocyte-specific receptors. In vertebrates, the integrin family contains 24 heterodimeric receptors that mediate attachment of cells to the ECM (Taken from Barczyk et al., 2010). 
Integrin signalling impacts the physiological function of all cells and tissues in the body. These receptors bind to ECM protein ligands via short amino acid sequences, such as the GFOGER motifs found in collagens (Knight et al., 2000), and the ArgGly-Asp (RGD) tripeptide sequence motifs present in several ECM proteins including; fibronectin, vitronectin and fibrinogen, in addition to collagens and laminins (Barczyk et al., 2010; Bellis, 2011). Furthermore, a single ECM ligand can have the ability to bind to one or more integrin heterodimer. The wide range of integrins that are expressed on a particular cell type and in different subcellular regions can be unique and can vary in a temporal manner.

Integrins function as mechanotransducers, transforming mechanical forces created by the ECM or the intracellular actin-cytoskeleton into biochemical signals (Larson et al., 2006). These receptors can function in a bi-directional manner across cell membranes. ECM ligands binding to integrin receptors can mediate intracellular signalling processes, which are termed as 'outside-in' signalling. In addition, intracellular signals originating within the cell cytoplasm can control integrin function, which can then be transmitted to the ECM ligand-binding domain; a process which is termed as 'inside-out' signalling. In 'inside-out' signalling, both an increase in integrin binding to ECM ligands, as well as the clustering of multiple integrin receptors in close proximity within the cell membrane occurs. It is understood that integrin clustering in both CMs and cFBs in the heart is associated with biochemical and mechanical signalling (Manso et al., 2009) (Figure 8). 
(A)

(B)
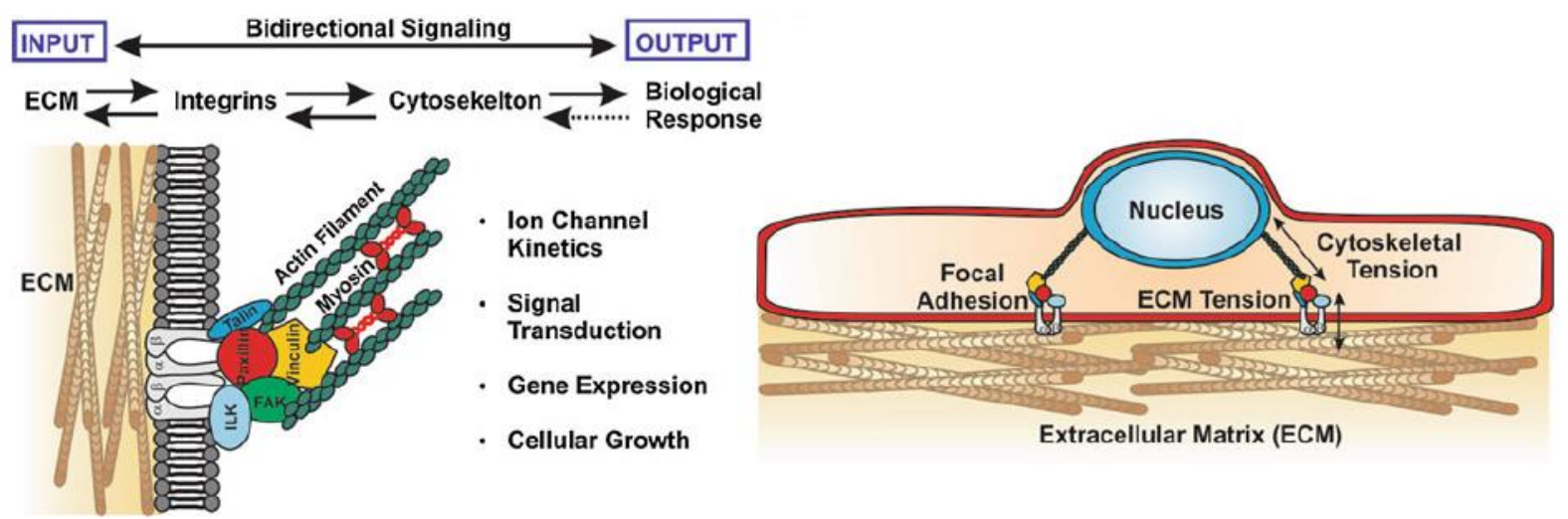

(C)
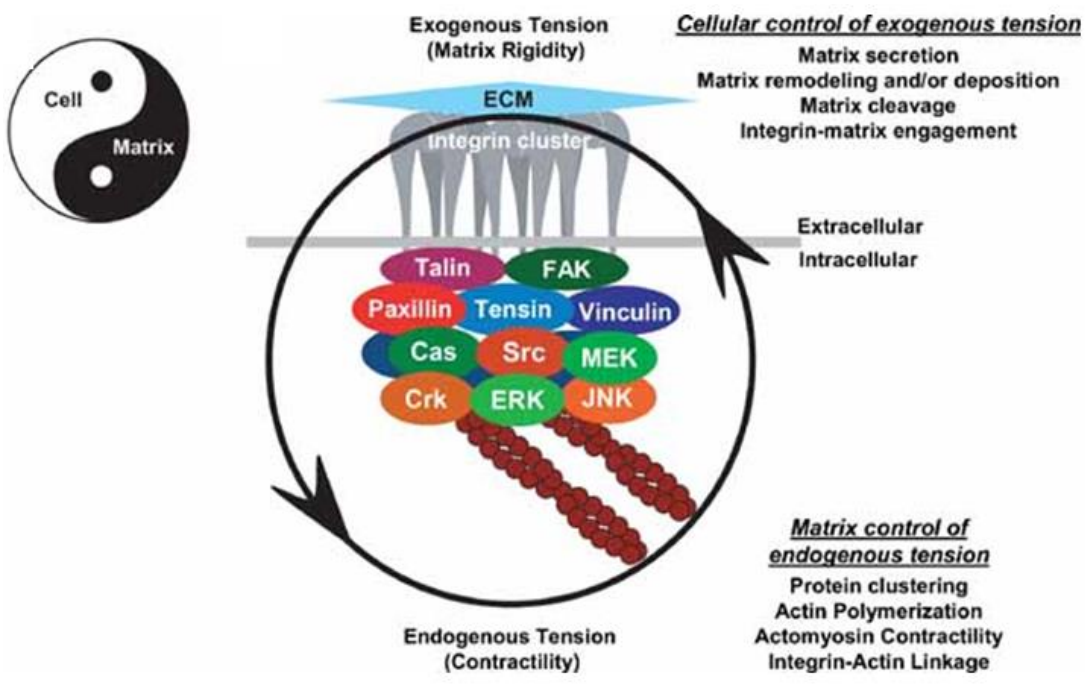

Figure 8. A simplified schematic of bi-directional ECM-cell signalling interfaces in the myocardium. (A) External mechanical cues are transmitted in a bi-directional manner from the ECM to intracellular compartments via transmembrane integrin receptors. These signals activate a number of biological responses, and in turn these biological responses can feed information back to the ECM via the same mechanical pathways. (B) Integrin receptors form a direct physical link between the ECM and the cytoskeleton through focal adhesions that translate mechanical signals directly into intracellular compartments such as the nucleus to regulate gene transcription. (C) Mutual bi-directional signalling between the ECM (exogenous tension can regulate endogenous cellular tension) and intracellular compartments (endogenous tension can directly or indirectly control ECM rigidity) through integrin receptors are necessary to regulate and maintain tension in the cellular microenvironment (Adapted from Sheehy et al., 2012 and Berrier and Yamada, 2007). 
This integrin bi-directional signalling is essential for cells to sense and respond to surrounding environmental changes with precise timing and kinetics, in order to maintain tissue integrity and homeostasis. Both cells and the ECM are able to mutually interact to regulate exogenous tension (ECM rigidly) and endogenous tension (cell actomyosin contractility) in the cellular microenvironment (Berrier and Yamada, 2007) (Figure 8C).

Mechanical forces are transmitted across the integrin-ECM interface to the cellular cytoskeleton, where they activate mechano-sensitive signal transducers. The signalling events critical in cell-ECM adhesion complexes involve a wide range of different cytoplasmic proteins (Lo, 2006). These include an array of different intracellular proteins such as: a) integrin-binding proteins; b) adaptor/scaffold proteins and c) enzymes. Integrin-binding proteins such as Talin directly bind to integrincytoplasmic domains and play a role in regulating integrin activation and signalling processes (Calderwood, 2004). Adaptor/scaffold proteins connect integrin-associated proteins with the actin-cytoskeleton, or an array of different other proteins including; paxillin, vinculin and $\alpha$-actinin. Finally, enzymes include non-receptor tyrosine kinases, such as focal adhesion kinases (FAK). Mechanosensitive signal transducers such FAK are able to translate a mechanical cue from the ECM environment into a biochemical response (Sheehy et al., 2012; Samarel, 2005; Schwartz and Ginsberg, 2002; Burridge and Chrzanowska-Wodnicka, 1996; Li et al., 2005). This transmitted information has been shown to activate a wide array of different downstream intracellular pathways, including PI3K, ERK, Src and MAP kinase pathways, which can modulate transcriptional activity and direct important cellular activities such as; cellular adhesion dynamics, the induction of apoptosis, cell cycle entry and regulate gene expression (Sheehy, 2012; Chen et al., 1997; Hynes, 2002; Schwartz et al., 1995; Fletcher and Mullins, 2010; Ingber, 2006).

The detailed understanding of these complex signalling pathways and networks is thus the ultimate goal to define the molecular basis of specific patterns in cardiac development and remodelling. 


\subsubsection{Integrin-mediated signalling in the myocardium}

Several studies have addressed the roles of integrins in the myocardium by genetic and biochemical approaches. The essential role of integrin-mediated signalling became apparent in integrin $\beta 1$ transgenic mice. Cardiac cells from chimeric mice constructed with a $\beta 1$ integrin-null allele exhibited delayed development and differentiation of cardiac lineage, as well as abnormal myofibrillogenesis (Fässler et al., 1996). Moreover, using integrin $\beta 1$ deficient embryonic stem cells for the in vitro differentiation of CMs, severely disrupted cardiomyogenesis and defective myofibrillogensis was observed (Fässler et al., 1996).

Furthermore, when integrin $\beta 1$ was inactivated exclusively in ventricular CMs, this resulted in myocardial fibrosis and heart failure (Shai et al., 2002). In addition, the disruption of integrin $\beta 1$ function in $\mathrm{CMs}$ by a high level of dominant-negative integrin $\beta 1$ mutant under the control of the CM-specific $\alpha$-myosin heavy chain $(\alpha \mathrm{MHC})$ promoter, displayed a more severe pathological phenotype. These transgenic mice die perinatally and display fibrotic replacement of the myocardium (Keller et al., 2001). More recently, it was demonstrated that during embryonic development embryonic cFBs produce a complex ECM environment (including collagens and fibronectin) which stimulated the proliferation of immature CMs through integrin $\beta 1$ signalling (Ieda et al., 2009). In addition, Ieda et al., were able to identify other FBsecreted growth factors which were involved in inducing CM proliferation; including heparin-binding epidermal growth factor-like growth factor (HB-EGF) (Ieda et al., 2009).

In summary, these studies indicate that cell-ECM interactions via integrin signalling are essential for the organization and maintenance of CMs in the myocardium during development. 


\subsection{Effects of the mechanical ECM environment on cardiomyocytes}

Cardiac organogenesis and pathogenesis are both characterized by changes in CM shape, cytoskeletal architecture, and the ECM. It is clear that mechanical forces of the ECM play critical roles in cardiac development of the embryonic heart, where mechanical forces are imposed on maturing CMs over the course of development (Jacot et al., 2010; Taber, 2001). These interactions between CMs and the ECM cause changes in cell shape that direct actin filament orientation, sarcomere organization and myofibrillogenesis in vitro (Sheehy et al., 2012; Bray et al., 2008). The ability of cells to move directionally towards areas of stiffer ECM via a process termed as 'durotaxis' is thought to be critical in influencing cellular phenotype and tissue morphogenesis (Plotnikov and Waterman, 2013). Studies on embryonic CMs when seeded on ECMs with different rigidity associated with normal cardiac development and fibrotic remodelling dramatically affected rhythmic contractility of the cells (Engler et al., 2006; Tobita et al., 2002).

It is clear that the mechanical properties of the cellular environment from the ECM contribute to the functional maturation of the myocardium. Our current knowledge of how changes in ECM organization and composition can influence structure and CM function is still limited. Obtaining mechanistic insights and understanding of how CMs respond to different ECM microenvironments may thus shed light into new targets which can support the improvement of cardiac regeneration therapies to treat cardiac diseases (van Spreeuwel et al., 2014). 


\subsection{Aims of Thesis}

It has been recognised that cFBs play an essential role in heart muscle development and homeostasis by establishing a supportive cardiac-microenvironment. We hypothesize that the mechanical and paracrine mediated effects of cFBs and its secreted ECM influence cardiac tissue formation and maturation. The underlying aims and objectives of this thesis were the following:

(1) To determine the mechanistic role of cFBs in mediating cardiac tissue formation and maturation by utilizing the established in vitro EHM model of heart muscle development.

(2) To identify the influence of cFB-derived ECM in controlling organotypic CM maturation.

(3) Elucidating the importance of collagen type I as a mediator of cardiac tissue formation and maturation by investigating how collagen processing HSP47 plays an essential role in cardiac muscle maturation and assembly into functional syncytia. 


\section{Materials and Methods}

\subsection{Cells}

All handling and experiments with cells were performed according to institutional regulations and good laboratory practice rules.

\subsubsection{Primary cell isolation and culture}

Mouse and human cells were utilized for all subsequent experiments. Mouse cells were isolated from primary tissue and human primary cells were purchased from commercially available sources.

\subsubsection{Mouse primary cells}

\subsection{Mouse embryonic fibroblast isolation}

Mouse embryonic fibroblasts (MEFs) were isolated from 10.5 to 13.5 postcoitum (dpc) mouse embryos (NMRI and C57B6/J) according to a modified cell isolation protocol (Conner, 2001). Briefly, pregnant mice were euthanized by cervical spine dislocation. Laparotomy was performed and uteri were harvested. The embryos were subsequently dissected from the uteri. The embryonic internal organs, including the head and liver were removed from the abdominal cavity. Embryos were rinsed in sterile $1 \mathrm{X}$ PBS and thereafter cut into 1-2 mm pieces using a sharp sterile pair of scissors. Minced embryos were transferred into a clean sterile Erlenmeyer flask containing glass beads (Pyrex $4 \mathrm{~mm}$; VWR, \#13782-554) and incubated in $25 \mathrm{ml}$ (per mouse) of pre-warmed $0.25 \%$ trypsin (Trypsin-EDTA) for 30 mins at $37{ }^{\circ} \mathrm{C}$, under gentle agitation on a magnetic stirrer. Trypsin was then deactivated by adding $\mathrm{x} 2$ volumes of MEF medium (see appendix A2) and allowed to stand for 3-5 mins at room temperature (RT) to allow the large tissue pieces to settle to the bottom of the flask. The cell suspension was removed (avoiding large tissue pieces) and subsequently transferred into fresh polypropylene tubes. Cells were centrifuged at 300 $\mathrm{x} g$ for 5 mins at RT, pelleted and resuspended in fresh pre-warmed MEF medium. 
Cells were finally plated into $15 \mathrm{~cm}$ culture plates (approximately one embryo per plate). MEF medium (20 ml) was changed every second day and maintained in culture until the specific confluency had been reached. MEFs from passages 1-5 were used for further experiments.

\subsection{Mitotic inactivation of fibroblasts with $\gamma$-irradiation}

MEFs and human forskin fibroblasts (hFFBs) were mitotically inactivated for use as a feeder layer for undifferentiated mouse (Conner, 2001) and human embryonic stem cells (Soong et al., 2012), respectively. Inactivated MEF and hFFB feeder layers provide a supporting growth environment for both mouse and human embryonic stem cells without dividing and diluting the cultivation of undifferentiated stem cells (Fuegemann et al., 2010; Wombus et al., 1991; Soong et al., 2012). To produce large stocks of inactivated MEFs, cells were expanded and grown until confluent (90-100 $\%$ ) onto twenty five to fifty $15 \mathrm{~cm}$ culture plates. hFFBs were cultured on T175 culture flasks and were similarly expanded and grown until confluent. MEFs or hFFBs were detached from plates/flasks (Section 2.1.2.1.5 and 2.1.2.2.3), resuspended in fresh growth medium (containing serum) and counted (Section 2.1.2.3.5). Cells were transferred into fresh $15 \mathrm{ml}$ polypropylene tubes $\left(2 \times 10^{6} / \mathrm{ml}\right)$ prior to irradiation. MEFs were then exposed to two individual cycles of 30 Gy from a $\gamma$-irradiation source (Steuerungstechnik und Strahlenschutz $\mathrm{GmbH}$ ) for 15 mins. hFFBs were exposed to only one cycle of $30 \mathrm{~Gy} \gamma$-irradiation. After irradiation, cell suspensions were counted again and frozen down into cryovial stocks $\left(8 \times 10^{6}\right.$ cells/ vial) accordingly (Section 2.1.2.3.4) until further use.

\subsection{Preparation of inactive MEF feeder layer plates}

For feeder layer preparation, culture plates were coated with gelatin to facilitate cell attachment. Firstly, $4 \mathrm{ml}$ of $0.1 \%$ Gelatin (Sigma, G2500; diluted in sterile water) was added sequentially to six $10 \mathrm{~cm}$ culture plates (Nunc). Plates were incubated for 20 mins at $37{ }^{\circ} \mathrm{C}, 5 \% \mathrm{CO}_{2}$. After, gelatin was aspirated and plates were allowed to air dry for 20 mins under a sterile laminar flow hood. A cryovial of inactivated MEFs (containing approximately $8 \times 10^{6}$ cells/ vial) was then thawed (Section 2.1.2.3.4). 
MEFs were resuspended in the appropriate amount of MEF medium and then transferred into the six $10 \mathrm{~cm}$ gelatin-coated plates. The feeder layer MEF plates could be used after $24 \mathrm{hrs}$ of cell plating. MEF plates were kept in culture for a maximum of 9 days.

\subsection{Neonatal mouse cardiac fibroblast isolation}

Neonatal mouse cardiac fibroblasts (cFBs) were isolated from neonatal mice 0-3 days postnatally (dpn), according to a modified cell isolation protocol (Simpson and Savion, 1982). Briefly, mice were decapitated the thoracic cavity was opened and the hearts were taken out. The atria were removed and the ventricles were cut into 1-2 mm pieces and washed twice in ice cold CBFHH buffer (see appendix A2). Tissues underwent alternating 3-4 min digestion cycles with Trypsin-working solution (see appendix A2) and DNase-working solution (see appendix A2) in CBFHH buffer (respectively) for over $4 \mathrm{hrs}$ at RT, with continuous agitation and gentle triturating to aid in cell dissociation. From the first digestion supernatant was discarded. Thereafter, with the subsequent remaining trypsin and DNase treatment steps, the cloudy supernatant was collected into collection tubes. These tryspin/DNase digestion cycles were repeated and supernatant was collected, until no more cells were released from tissues. Dissociated cells were then gently centrifuged at $60 \mathrm{x}$ g for $15 \mathrm{mins}$ and $4{ }^{\circ} \mathrm{C}$, pelleted and resuspended in $30 \mathrm{ml}$ ice cold MEF medium with the addition of $250 \mu \mathrm{l}$ DNase I $(1 \mathrm{mg} / \mathrm{ml})$. Cells were centrifuged again at $60 \mathrm{x} \mathrm{g}$ for $15 \mathrm{mins}$ at $4{ }^{\circ} \mathrm{C}$, pelleted and resuspended in fresh MEF medium, passed through a pre-wetted stainless steel sieve $(250 \mu \mathrm{m}$ pore size $)$ and then transferred into a fresh polypropylene tube. Cells were counted by mixing a small volume of cell suspension with $0.4 \%$ trypan blue solution (1:1 mixture) and then transferred into a Neubauer cell counting chamber to determine the number and viability of cells. To separate cardiomyocytes (CMs) from the non-myocyte cell fractions, the dissociated cells underwent a further pre-plating step. Cells were seeded $\left(1 \times 10^{7}\right.$ cells $/ 15 \mathrm{~cm}$ plate) and incubated in MEF medium for 60 mins at $37{ }^{\circ} \mathrm{C}, 5 \% \mathrm{CO}_{2}$. The growth medium contained the majority of CMs and this was then collected and replaced with pre-warmed MEF medium. The plates containing the majority of adherent $\mathrm{cFBs} /$ non-myocytes were replaced with fresh MEF medium every second day to wash off cellular debris. The cFBs were 
maintained in culture until the specific confluency had been reached. cFB from passages 1-3 were used for further experiments.

\subsection{Passaging of primary mouse fibroblasts}

Primary FBs (MEFs and cFBs) were routinely passaged when reaching full confluency (80-90\%). For passaging, cells were rinsed with an appropriate amount of PBS (pre-warmed at $\left.37^{\circ} \mathrm{C}\right)$ than treated with pre-warmed Trypsin $(0.25 \%$ TrypsinETDA; Invitrogen, 25200) for $2-5$ mins at $37{ }^{\circ} \mathrm{C}$, to facilitate cell detachment. Cell detachment from culture plates was controlled with a light microscope. The trypsin was subsequently deactivated by adding $\mathrm{x} 2$ volumes of MEF medium and triturated to produce a single cell suspension. Cells were thereafter pelleted at $300 \mathrm{x} \mathrm{g}$ for 5 mins and resuspended in fresh MEF medium. Cells were then counted (Section 2.1.2.3.5) and seeded at the required density on fresh culture plates or frozen down (Section 2.1.2.3.4).

\subsubsection{Human primary cells}

\subsection{Human foreskin fibroblasts}

Human foreskin fibroblasts (hFFBs) cells were purchased from the American Type Culture Collection (SCRC-1041; ATCC). hFFBs were cultivated in HEF medium (see appendix A2) and passages between P18-28 were used for all subsequent experiments.

\subsection{Human cardiac fibroblasts}

Human adult cardiac fibroblast cells (hcFB) were purchased from Lonza (V26C25). These cells were obtained from a middle aged male. hcFBs were cultivated in HEF medium (see appendix A2) and passages between P3-6 were used for all subsequent experiments. 


\subsection{Passaging and culture of primary human fibroblasts}

hFFBs and hcFBs were routinely passaged as described in section 1.2.3.5, with minor changes. Pre-warmed TrypLE (Invitrogen, 12604) was used for human FB cell detachment instead of $0.25 \%$ Trypsin-ETDA. After cell detachment, TrypLE was deactivated by adding $\mathrm{x} 2$ volumes of HEF medium and triturated to produce a single cell suspension. Cells were then counted and seeded at the required density on fresh non-coated culture plates or frozen down (Section 2.1.2.3.4).

\subsection{Freezing and thawing of primary mouse and human fibroblasts.}

Cultivated mouse FBs (MEFs; neonatal cFBs) and/or human FBs (hFFBs and hcFBs) were frozen down for practical storage purposes. Briefly, mouse and human cells were rinsed with pre-warmed PBS and then detached from culture plates into single cell suspensions (Sections 2.1.2.1.5 and 2.1.2.2.3), respectively. Cells were counted using a CASY counter (2.1.2.3.5), followed by centrifugation at $300 \mathrm{x}$ g for $5 \mathrm{mins}$ at RT. Cells were subsequently resuspended $\left(1 \times 10^{6}\right.$ to $8 \times 10^{6}$ cells $\left./ \mathrm{ml}\right)$ in freezing medium (see appendix A2) and gently mixed by trituration. Cells were finally transferred into cryovials $(1 \mathrm{ml} / \mathrm{vial})$, which were then immediately placed into an isopropanol insulated container at $-80{ }^{\circ} \mathrm{C}$, in order to freeze cells down slowly enough to maintain viability. Cells were able to be stored for years at $-80{ }^{\circ} \mathrm{C}$ or $-152{ }^{\circ} \mathrm{C}$. For cell thawing, cells were thawed rapidly by placing cryovials into a $37{ }^{\circ} \mathrm{C}$ water bath. Thawed cells were immediately transferred into polypropylene tubes with $9 \mathrm{ml}$ growth medium. Cells where then centrifuged at $300 \mathrm{x}$ g for $5 \mathrm{mins}$ at RT and resuspended in either; MEF (for mouse FBs) or HEF (for human FBs) medium, in order to remove the contaminating DMSO. Cell suspensions were then able to be used for subsequent experiments.

\subsection{Cell count and size measurements}

Cell number, size and viability measurements from single cell suspensions were assessed using a CASY Model TT cell counter (Roche), according to the manufacturer's instructions. A Neubauer cell counting chamber however was used to 
determine the number of total viable single cells after the neonatal mouse heart isolation (Section 2.1.2.1.4).

\subsubsection{Embryonic stem cell generation and culture}

\subsubsection{Mouse embryonic stem cells}

The mouse CM-selectable embryonic stem cell (mESC) line ( $\alpha$ MHC-neoR “A6-line”; R1 background) was generated by electroporation of a plasmid encoding for a neomycin resistance gene (neoR), under the control of the cardiomyocyte restricted $\alpha$ myosin heavy chain ( $\alpha \mathrm{MHC}$ ) promoter (Rogge, Dissertation 2007), as described previously (Klug et al., 1996).

\subsection{Culture of mouse embryonic stem cells}

Mouse ESCs were cultured on mitotically inactive MEFs $\left(25,500\right.$ cells $/ \mathrm{cm}^{2}$; Section 2.1.2.1.3) in ESC culture medium (see appendix A2). Medium was exchanged every day. Once mESC-colonies reached 80-90 \% confluency, cells were detached (Section 2.1.2.1.5) with $0.25 \%$ trypsin-EDTA and split at a 1:5 ratio. mESC-colonies were seeded onto new MEF feeder layer plates in ESC medium. Undifferentiated mESCs were cultured for at least 3 passages, prior to the initiation of cardiac differentiation.

\subsection{Scale up differentiation of mouse $\alpha$ MHC-NeoR ESCs}

The differentiation of mESCs was scaled up into spinner flasks, as described previously (Christalla, Dissertation 2010; Niebruegge et al., 2008). Undifferentiated mESCs were dissociated into single cells with trypsin (Section 2.1.2.1.5). Cells were resuspended in differentiation medium (see appendix A2) and pre-plated for 40 mins to minimize the MEF content. Cell suspensions were subsequently counted with a Neubauer cell counting chamber. Spinner flask culture vessels (125 ml) equipped with a bulb-shaped glass stirrer (Techne, F7988) were inoculated with $50 \mathrm{ml}$ of medium containing $10 \times 10^{6}$ cells and stirred at $60 \mathrm{rpm}$ in a humidifying chamber at $37^{\circ} \mathrm{C}$ in a 
$5 \% \mathrm{CO}_{2}$ incubator. Flasks were filled up to $100 \mathrm{ml}$ differentiation medium after 24 hrs, followed by half medium exchange every $48 \mathrm{hrs}$. CM selection at day 11 was initiated by the addition of $400 \mu \mathrm{g} / \mathrm{ml}$ Geneticin (G418, PAA) for a further 6 days, in order to eliminate non-myocytes.

\subsection{Digestion of embryoid bodies into single cardiomyocytes}

At culture day 17, beating embryoid bodies (EBs) were harvested and dissociated into single CMs. EBs were transferred into two clean $50 \mathrm{ml}$ polypropylene tubes. The tubes were left to rest for approximately 5-10 mins to allow the EBs to settle to the bottom of the tubes. The supernatant was very carefully removed and EBs were washed with pre-warmed 1X PBS and pelleted at $300 \mathrm{x} \mathrm{g}$ for 5 mins at RT. The supernatant again was carefully removed and EBs were resuspended in $6 \mathrm{ml}$ Collagenase type I (Sigma-Aldrich, C0130; see appendix A2), containing DNase I (20 $\mu \mathrm{g} / \mathrm{ml}$; Calbiochem). The EBs were triturated and then incubated at $37{ }^{\circ} \mathrm{C}$ for $60-90$ mins with agitation on a mechanical rocking platform (Biometra) to dissociate the EBs and yield single cells. Once all the EBs were dissociated, each tube was filled with pre-warmed $1 \mathrm{X}$ PBS and pelleted at $500 \mathrm{x} \mathrm{g}$ for $5 \mathrm{mins}$ at RT. Cells were then resuspended in pre-warmed $6 \mathrm{ml} 0.25 \%$ Trypsin-ETDA and were gently triturated for 5-10 mins until all cell clumps were dissociated. The enzymatic digestion was inactivated with $24 \mathrm{ml}$ differentiation medium + DNase I $(20 \mu \mathrm{l} / \mathrm{ml})$. The cell suspensions were then passed through a pre-wetted $70 \mu \mathrm{m}$ cell strainer (BD falcon) into a fresh $50 \mathrm{ml}$ polypropylene tube. The cells were pelleted at $200 \mathrm{x} \mathrm{g}$ for $5 \mathrm{mins}$ at $4{ }^{\circ} \mathrm{C}$, resuspended in $10 \mathrm{ml}$ differentiation medium and the tubes were pooled together. Cells were counted with (1:1) $0.4 \%$ trypan blue solution using a Neubauer cell counting chamber to determine the number and viability of cells. Cells were subsequently used for experiments, with a small cell fraction $\left(\sim 1 \times 10^{6}\right.$ cells $)$ fixed in $70 \%$ ethanol $(\mathrm{EtOH})$ to assess the purity of $\mathrm{CM}$ via flow cytometry (FC) analysis (Section 2.1.4). 


\subsubsection{Human embryonic stem cells}

Experimentation with human embryonic stem cells were approved by the RobertKoch-Institute (www.rki.de; approval \#12 from 13.09.2005 to W.H. Zimmermann according to $§ 11$ Stammzellgesetz) and the Human Embryonic stem cell (hES2) line was obtained from the lab of Prof. Gordon Keller, Toronto, Canada. hES2 line contained a targeted red fluorescence protein (tdRFP) reporter gene to the human ROSA26 locus (Irion et al., 2007). hES2 cells were initially adapted to culture with hESC medium (see appendix A2) on $\gamma$-irradiated hFFBs, prior to cardiac differentiation (Soong et al., 2012; Zimmermann et al., 2015). hESC-medium was exchanged daily until colonies covered $80 \%$ of the culture flasks.

\subsubsection{1 hES2 2D cardiac differentiation}

Cardiac differentiation in 2D culture was performed on hES2 cells using an optimized protocol (Hudson et al., 2012). All 2D differentiation experiments were performed in 24 well plates filled with $0.5 \mathrm{ml}$ culture medium and cultivated at $37^{\circ} \mathrm{C}$ in a $5 \% \mathrm{CO}_{2}$ incubator. Briefly, hES2 cells were plated at $5 \times 10^{4}$ cells $/ \mathrm{cm}^{2}$ on Matrigel (growth factor reduced)-coated plates (1:60 diluted in 1X PBS; BD Biosciences, 354320) and cultured in 1:1 with hES-medium and irradiated hFFB-conditioned medium (conditioned for 2 days) with $10 \mathrm{ng} / \mathrm{ml}$ FGF2 (seeding phase). After $24 \mathrm{hrs}$ seeding, hES2 cells were rinsed with RPMI medium (see appendix A2) and subsequently cultured in Mesoderm Induction medium (see appendix A2) for 3 days, followed by Cardiac Specification medium (see appendix A2) for 8 days, and thereafter Maturation medium for 1 day (see appendix A2). Differentiated cells were then cultivated for 5 days in Selection medium $\left(0.28 \mathrm{ml} / \mathrm{cm}^{2}\right.$; see appendix A2) to enrich the $\mathrm{CM}$ populations and eliminate non-myocytes via metabolic selection (Tohyama et al., 2013). Cells were then cultivated for a further 7 days in Maturation medium. 


\subsection{Single cell dissociation of hES2-cardiomyocyte monolayers}

Enriched CM populations derived from hES2 cardiac differentiation in monolayer culture (Section 2.1.3.2.1) were dissociated into single cells. Cells were washed twice in 1X PBS. Next, cells were incubated with Accutase solution (See appendix A2; 0.1 $\mathrm{ml} / \mathrm{cm}^{2}$ ) for 4 mins at RT. Cells were then incubated for a further $10-15$ mins at $37{ }^{\circ} \mathrm{C}$, until cells were detached from culture flasks and dissociated into single cells. The digestion procedure was deactivated with the addition of a threefold amount of serumfree CM medium containing $5 \mu \mathrm{mol} / 1$ Rock inhibitor (see appendix A2). Cells were triturated to aid in single cell dissociation, transferred into fresh polypropylene tubes and counted using the CASY counter (Section 2.1.2.3.5) to determine cell number and viability. Cells were subsequently used for experiments, with a small cell fraction $(\sim 1$ $\mathrm{x} 10^{6}$ cells) fixed in $70 \%$ ethanol $(\mathrm{EtOH})$ to assess the purity of $\mathrm{CMs}$ via Flow cytometry analysis (Section 2.1.4).

\subsubsection{Flow cytometry}

Flow cytometry (FC) was used to assess the purity of CMs derived from mESCs or hES2 cells. Fixed cell suspensions (in 70\% EtOH) were initially passed through a 70 $\mu \mathrm{m}$ cell strainer (to remove cell clumps), pelleted and then permeabilized in blocking buffer solution (see appendix A2) for 10 mins at RT. Cells were then incubated with primary antibody against CM-specific $\alpha$-sarcomeric actinin (Sigma-Aldrich, A7811, 1:4000) for 45 mins at $4{ }^{\circ} \mathrm{C}$. Cell populations in parallel were also incubated with a primary antibody against Immunoglobulin $\mathrm{G}(\mathrm{IgG})$ isotype control (R\&D systems), which served in samples as the respective negative control. Fixed cell suspensions were then washed (x2) in blocking buffer solution for 3 mins, followed by incubation with the Goat anti-Mouse Alexa 488 secondary antibody (Invitrogen, A-11001; 1: 1000) and the nuclei labelling dye Hoechst (Invitrogen, H2570; 1: 1000) for $1 \mathrm{hr}$ at 4 ${ }^{\circ} \mathrm{C}$ in the dark. Cells were then again washed (x2) in blocking buffer for 3 mins. Cells were finally resuspended in PBS and cell populations were assessed on a LSRII cytometer (BD) and analyzed on FACSDiva Software 6.0 (BD). 


\subsection{Generation of Engineered Heart Muscle (EHM)}

Engineered Heart Muscle (EHM) are collagen-hydrogel based constructs comprising of the most abundant cellular and ECM components of native heart muscle, which resemble structural and functional properties of the native myocardium (Tiburcy et al., 2011; Zimmermann et al., 2002).

\subsubsection{Construction and culture of EHMs}

Mouse and human EHMs were generated as described previously with minor modifications (Didié et al., 2013; Tiburcy et al., 2014). Custom-made culture dishes consisting of 4 ring-shaped molds (Figure 9) were used for casting mouse and human EHMs.
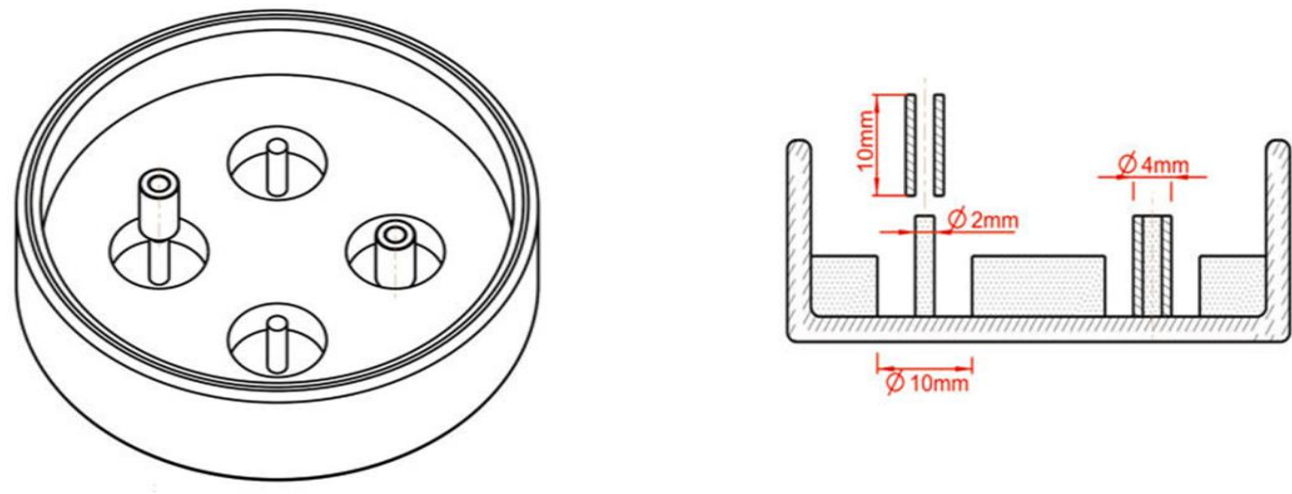

Figure 9. Design of casting molds for mouse and human EHM culture. EHM glass culture dishes consisted of 4 transparent silicon ring-shaped molds. The cylindrical silicon tubing in the middle was used as an inner central spacer during the first 3 days of the EHM casting/formation process. The inner diameter of molds was $4 \mathrm{~mm}$, and outer diameter $10 \mathrm{~mm}$ (Images taken from Tiburcy et al., 2014).

All pipetting steps were performed on ice to prevent premature polymerization of the EHM reconstitution mixture. Firstly, single cell suspensions (from mouse or human) containing $75 \%$ of $\mathrm{CM}$ and $25 \% \mathrm{FB}$ cell fractions, were resuspended in the suitable volume of differentiation medium (cell suspension) to generate the respective number of EHMs (Table 1). Next, equal amounts of $2 \mathrm{X}$ concentrated medium (2X DMEM) (containing; FCS [40 \%]; 10X DMEM [20\%]; P/S [2\%] in deionised water; sterile 
filtered through a $0.22 \mu \mathrm{m}$ syringe filter) were separately added to rat or bovine collagen type I. The yellow/orange colour change was an indicator for the acidity in the mixture. The $\mathrm{pH}$ was then adjusted (approx. 7.4) with the drop wise addition of $0.1 \mathrm{~N} \mathrm{NaOH}$, as indicated by the pink colour change. The cell suspension was then added to the mixture and triturated several times to aid in homogeneity of the EHM reconstitution mixture. Finally, $450 \mu \mathrm{l}$ of EHM mixture was distributed into individual casting mold recesses and incubated at $37{ }^{\circ} \mathrm{C}$ in a humidified incubator with $5 \% \mathrm{CO}_{2}$ for $1 \mathrm{hr}$.

\begin{tabular}{|l|lll|}
\hline Mouse EHM master mix components & \multicolumn{3}{|l|}{ Number of mEHMs } \\
\hline Rat tail collagen $(\mathbf{4 . 2} \mathbf{~ m g / m l )}$ & $\mathbf{x 1}$ & $\mathbf{x 4}$ & $\mathbf{x 8}$ \\
$\mathbf{2 x}$ DMEM & $100 \mu \mathrm{l}$ & $400 \mu \mathrm{l}$ & $800 \mu \mathrm{l}$ \\
$\mathbf{0 . 1}$ N NaOH & $100 \mu \mathrm{l}$ & $400 \mu \mathrm{l}$ & $800 \mu \mathrm{l}$ \\
Cell suspension* & $20 \mu \mathrm{l}$ & $80 \mu \mathrm{l}$ & $150 \mu \mathrm{l}$ \\
Total volume of master mix & $306 \mu \mathrm{l}$ & $1224 \mu \mathrm{l}$ & $2448 \mu \mathrm{l}$ \\
\hline
\end{tabular}

\begin{tabular}{|c|c|c|c|}
\hline \multirow[t]{2}{*}{ Human EHM master mix components } & \multicolumn{3}{|c|}{ Number of hEHMs } \\
\hline & $\mathbf{x 1}$ & $\mathrm{x4}$ & $\mathrm{x8}$ \\
\hline Bovine collagen $(7.25 \mathrm{mg} / \mathrm{ml})$ & $65 \mu 1$ & $258 \mu 1$ & $515 \mu 1$ \\
\hline 2X DMEM & $65 \mu 1$ & $258 \mu 1$ & $515 \mu 1$ \\
\hline $0.1 \mathrm{~N} \mathrm{NaOH}$ & $13 \mu 1$ & $50 \mu 1$ & $100 \mu \mathrm{l}$ \\
\hline Cell suspension* & $384 \mu 1$ & $1535 \mu 1$ & $3070 \mu 1$ \\
\hline Total volume of master mix & $527 \mu 1$ & $2110 \mu 1$ & $4200 \mu 1$ \\
\hline
\end{tabular}

Table 1. Composition of EHM mastermix. The respective total cell numbers in 'cell suspension (*)' for different amounts of EHM mastermix: x1 (1.5 x10 cells); x4 (6 x 10 cells); x8 (12 x10 cells), in mouse (mEHM) and human (hEHM). The final collagen concentration was $0.4 \mathrm{mg} / \mathrm{EHM}$.

After $1 \mathrm{hr}$ EHM condensation, $6 \mathrm{ml}$ of pre-warmed differentiation medium (see appendix A2) was added to each EHM casting dish 24 hrs. Images of EHM condensation on casting molds over the first 3 days of culture were taken using the Lumar V12 microscope (Zeiss) connected to the AxioCam (MRC) camera. On day 3, 
the EHMs were progressively condensed and were transferred onto custom mechanical (static) stretchers (Figure 10) on 6 well plates. EHMs were maintained in culture for an additional 7 days. Medium was exchanged every $48 \mathrm{hrs}$.

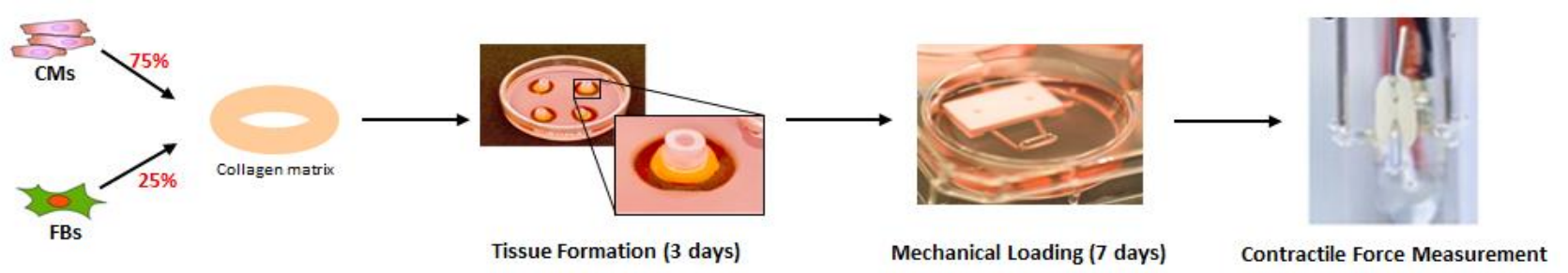

Figure 10. Schematic overview outlining the formation and assessment of EHMs. EHM generation, tissue formation, mechanical (static) loading and functional (contractile force) measurements.

\subsubsection{Isometric force measurement}

Isometric force measurements of EHMs were performed as previously described (Zimmermann et al., 2000). Briefly, EHMs after a total of 10 days culture were carefully transferred into hooks of a force transducer in organ baths filled with Tyrodes solution (see appendix A2), maintained at $37{ }^{\circ} \mathrm{C}$ and perfused with carbogen $\left(\mathrm{pH} 7.4,95 \% \mathrm{O}_{2}, 5 \% \mathrm{CO}_{2}\right)$. EHMs were electrically stimulated via two platinum electrodes at $4 \mathrm{~Hz}$ (5 ms monophasic pulses, $200 \mathrm{~mA})$. EHMs were pre-stretched under $1.8 \mathrm{mmol} / \mathrm{L}\left[\mathrm{Ca}^{2+}\right]$ until the force of contraction reached a stable level $\left(\mathrm{L}_{\max }\right)$. After adjustment of extracellular $\mathrm{Ca}^{2+}$ to $0.2 \mathrm{mmol} / \mathrm{L}$, the contractile forces of EHMs were measured in response to a stepwise increase of extracellular $\left[\mathrm{Ca}^{2+}\right](0.2-2.4$ $\mathrm{mmol} / \mathrm{L})$. The maximum and minimum forces were evaluated using BMON software (G. Jaeckel, Hanau) and analysed by AMON software (G. Jaeckel, Hanau). Force of contraction was calculated by the difference between maximum and minimum forces. 


\subsubsection{Whole mount Immunoflourescence imaging}

Whole mount immunoflourescence imaging was performed to visualize cardiac muscle formation within EHMs. After cultivation, EHMs were washed once on $1 \mathrm{X}$ PBS and fixed in Roti ${ }^{\circledR}$ Histofix $4 \%$ overnight at $4{ }^{\circ} \mathrm{C}$. Thereafter, EHMs were washed once in 1X PBS and incubated in permeabilizing blocking buffer solution (see appendix A2) for $24 \mathrm{hrs}$ at $4{ }^{\circ} \mathrm{C}$. EHMs were subsequently incubated with a mouse monoclonal primary antibody targeting alpha sarcomeric $\alpha$-actinin (1:1000; Table 2) for $48 \mathrm{hrs}$ on a rotary microfuge holder at $4{ }^{\circ} \mathrm{C}$. EHMs were then washed (x3) in blocking buffer for 15 min incubation steps, followed by the incubation with the Goat anti-Mouse Alexa 488 secondary antibody (1: 400; Table 2) secondary antibody, Factin labelling dye, phalloidin (conjugated with Alexa Fluor® 633, 1:60; Table 2) and the nuclei labelling dye, Hoechst (1:1000; Table 2) for a further $24 \mathrm{hrs}$ on the rotary microfuge holder at $4{ }^{\circ} \mathrm{C}$ in the dark. EHMs were again thoroughly washed (x3) in blocking buffer for 15 min incubation steps and were thereafter stored in x1 PBS. Finally, EHMs were mounted onto microscope slides using Fluoromount-G mounting medium (Southernbiotech). Microscope slides were imaged using the Zeiss LSM710 confocal microscope.

\subsection{Integrin expression in fibroblast and cardiomyocyte populations}

\subsubsection{Protein biochemical analysis}

Cultivated FBs and CMs were rinsed thoroughly in 1X PBS to remove any remaining medium/serum and cell debris. The following protein extraction (Section 2.3.1.2) or RNA extraction (Section 2.3.2.1) steps on cell lysates were then performed as described below.

\subsubsection{Protein extraction}

To obtain cell lysates from cultured cells, CytoBuster ${ }^{\mathrm{TM}}$ Protein extraction reagent (Millipore; 71009; containing both Protease [Roche] and Phosphotase (PhosSTOP) inhibitor [Roche] cocktails), was layered over the cells (300 $\mu 1 /$ well in 6 well plates) 
and allowed to extract for 5 mins at $4{ }^{\circ} \mathrm{C}$ (according to manufacturer's instructions). Thereafter, a cell scraper (Sarstedt) was used to collect the lysated cells to be transferred into $1.5 \mathrm{ml}$ microfuge tubes. Cell lysates were centrifuged at $14,000 \mathrm{x} \mathrm{g}$ for 5 mins at $4{ }^{\circ} \mathrm{C}$ to pellet the cell debris. The supernatant containing cell lysates were separated from the bottom cell debris pellet, collected into sterile microfuge tubes and stored at $-20^{\circ} \mathrm{C}$ until further use.

\subsubsection{Protein quantification (Bradford assay)}

The concentration of total protein in cell lysate samples was determined using a standard colorimetric Bradford protein assay (Bradford, 1976). Briefly, samples (thawed and stored on ice) and a standard curve with increasing protein BSA amounts $(0,1,2,4,8,16 \mu \mathrm{g})$ were prepared in $50 \mu \mathrm{L}$ distilled $\mathrm{H}_{2} \mathrm{O}$. Samples and standards were prepared in triplicates and transferred into a 96-well microplate. Into each sample and standard triplicate, $200 \mu \mathrm{L}$ of 1x Bradford reagent (Roti Quant; K015.3) in $\mathrm{H}_{2} \mathrm{O}$ was added and incubated for $5 \mathrm{~min}$ at RT. The absorbance at $595 \mathrm{~nm}$ was detected with the Flexstation ${ }^{\circledR} 3$ Multi-mode microplate reader (Molecular Devices) and the total protein concentrations of the samples were calculated from standard curves.

\subsubsection{Immunoblotting analysis}

For analysis of protein expression in total cell lysates, sodium dodecyl sulfate polyacrylamide gel electrophoresis (SDS-PAGE) was performed. To prepare SDSPAGE samples, 6X Laemmli Loading buffer (see appendix A2) was added to cell lysates $(15 \mu \mathrm{g} / \mathrm{sample})$ and incubated at $95{ }^{\circ} \mathrm{C}$ for $5 \mathrm{~min}$ for protein denaturation.

Proteins were loaded on 10-15\% SDS-polyacrylamide gels (see appendix A2) and separated electrophoretically. The Precision Plus ${ }^{\mathrm{TM}}$ Protein Kaleidoscope ${ }^{\mathrm{TM}}$ protein ladder (BioRad) was used for the estimation of molecular weights in all protein samples. Protein samples were ran in 1x electrophoresis running buffer (appendix A2) at $70 \mathrm{~V}$ for initial sample condensation and later at $120 \mathrm{~V}$ for protein separation. Thereafter, resolved SDS-PAGE gels were electrophoretically transferred onto 
activated polyvinylidene fluoride (PVDF) membranes (Amersham ${ }^{\mathrm{TM}}$ Hybond $^{\mathrm{TM}} \mathrm{P}$ $0.45,10600023$ ) and incubated in $1 \mathrm{x}$ transfer buffer (appendix A2) at $4{ }^{\circ} \mathrm{C}$ for $1 \mathrm{hr}$ at 100 V. All membrane blots were blocked with $10 \%$ Roti ${ }^{\circledR}$-block blocking solution (diluted in $\mathrm{dd}_{2} \mathrm{O}$; Roth, A151.4) on a mechanical rocking platform (Biometra) for 1 $\mathrm{hr}$ at RT, followed by incubation with the respective primary antibodies (Table 2) prepared in TBST buffer (see appendix A2) overnight at $4{ }^{\circ} \mathrm{C}$. Thereafter, the membranes were thoroughly washed (x3) in TBST buffer for 10 mins and incubated with the following secondary antibodies (Table 2) on the rocking platform for $1 \mathrm{hr}$ and RT. To visualize membrane bands, protein-antibody complexes were detected using the chemiluminescent reagent SuperSignal ${ }^{\circledR}$ West Femto Maximum Sensitive Substrate (Thermo Scientific, \# 34095). The signals were detected with a ChemDoc ${ }^{\mathrm{TM}}$ MP Imaging System (BioRad) and semi-quantitatively analyzed with Image Lab ${ }^{\mathrm{TM}}$ Software v5.1 (BioRad). 


\begin{tabular}{|c|c|c|c|c|c|}
\hline \multicolumn{2}{|c|}{ Primary Antibody } & \multicolumn{3}{c|}{ Dilution/Amount } \\
\hline Name & Species & Manufacturer/Catalogue no. & IHC & WB & FACS \\
\hline anti-Calsequestrin & Rabbit & ThermoFisher Scientific (PA1-913) & - & $1: 1000$ & - \\
\hline anti-Cardiac Troponin I & Rabbit & Abcam (ab47003) & $1: 200$ & $1: 200$ & - \\
\hline $\begin{array}{c}\text { anti-Collagen (pro-) type I } \\
\text { aminopropeptide) }\end{array}$ & Mouse & DSHB (SP1.D8) & - & $0.5 \mu \mathrm{g} / \mathrm{ml}$ & - \\
\hline anti-Collagen type I & Rabbit & Abcam (ab34710) & $1: 200$ & - & - \\
\hline anti-FSP-1 & Rabbit & Millipore (ABF32) & - & $1.0 \mu \mathrm{g} / \mathrm{ml}$ & - \\
\hline anti-GAPDH & Mouse & Millipore (MAB374) & - & $1: 1000$ & - \\
\hline anti-HSP47 & Mouse & Enzo Life Science (SPA470) & $1: 100$ & $1: 1000$ & - \\
\hline $\begin{array}{c}\text { anti-Mouse IgG } \text { isotype } \\
\text { control }\end{array}$ & Mouse & R\&D Systems (MAB002) & $1: 100$ & - & $1: 1000$ \\
\hline anti-Integrin alpha 1 & Rabbit & Abcam (ab106267) & - & $2.0 \mu \mathrm{g} / \mathrm{ml}$ & - \\
\hline anti-Integrin alpha 2 & Rabbit & Abcam (ab133557) & - & $1: 10,000$ & - \\
\hline anti-Integrin beta 1 & Rabbit & Abcam (ab179471) & - & $1: 2000$ & - \\
\hline anti-PDI & Rabbit & Cell Signalling (\#2446) & $1: 50$ & $1: 1000$ & - \\
\hline anti-Pro-COL1A1 & Rabbit & Santa Cruz (sc-30136) & $1: 100$ & - \\
\hline anti-Sarcomeric $\alpha$-actinin & Mouse & Sigma-Aldrich (A7811) & $1: 1000$ & - & $1: 4000$ \\
\hline
\end{tabular}

\begin{tabular}{|c|c|c|c|c|c|}
\hline \multicolumn{2}{|c|}{ Secondary Antibody } & \multicolumn{3}{c|}{ Dilution/Amount } \\
\hline Name & Species & Manufacturer/Catalogue no. & IHC & WB & FACS \\
\hline $\begin{array}{c}\text { anti-Mouse IgG/HRP } \\
\text { conjugate }\end{array}$ & Goat & Dako (P0260) & - & $1: 10,000$ & - \\
\hline $\begin{array}{c}\text { anti-Rabbit IgG/HRP } \\
\text { conjugate }\end{array}$ & Goat & Dako (P0448) & - & $1: 5000$ & - \\
\hline $\begin{array}{c}\text { anti-Mouse-Alexa 488 } \\
\text { anti-Rabbit-Alexa 488 }\end{array}$ & Goat & Invitrogen (A11001) & $1: 400$ & - & $1: 1000$ \\
\hline anti-Mouse-Alexa 546 & Goat & Invitrogen (A11008) & $1: 400$ & - & - \\
\hline anti-Rabbit-Alexa 546 & Goat & Invitrogen (A11003) & $1: 400$ & - & - \\
\hline
\end{tabular}

\begin{tabular}{|c|c|c|c|c|c|}
\hline \multicolumn{2}{|c|}{ Fluorescent Conjugate } & \multicolumn{3}{c|}{ Dilution/Amount } \\
\hline Name & Species & Manufacturer/Catalogue no. & IHC & WB & FACS \\
\hline Alexa Flour Phalloidin 633 & - & ThermoFisher Scientific (A22284) & $1: 60$ & - & - \\
\hline Hoechst & - & Invitrogen (H3570) & $1: 1000$ & - & $1: 1000$ \\
\hline
\end{tabular}

\begin{tabular}{|c|c|c|c|}
\hline \multicolumn{2}{|c|}{ Blocking Antibody } & Dilution/Amount \\
\hline Name & Species & Manufacturer/Catalogue no. & EHM blocking \\
\hline $\begin{array}{c}\text { anti-Rat/Mouse CD49a } \\
\text { (Integrin alpha 1 chain) }\end{array}$ & Hamster & BD Pharmingen $^{\mathrm{TM}}(555001)$ & $50 \mu \mathrm{g} / \mathrm{ml}$ \\
\hline $\begin{array}{c}\text { anti-Rat CD49b (Integrin alpha } \\
2 \text { chain) }\end{array}$ & Hamster & BD Pharmingen $^{\mathrm{TM}}(554998)$ & $50 \mu \mathrm{g} / \mathrm{ml}$ \\
\hline $\begin{array}{c}\text { anti-Rat CD29 (Integrin beta 1 } \\
\text { chain) }\end{array}$ & Hamster & BD Pharmingen $^{\mathrm{TM}}(555002)$ & $40 \mu \mathrm{g} / \mathrm{ml}$ \\
\hline
\end{tabular}

Table 2. List of primary and secondary antibodies/fluorescent conjugates used for protein biochemical analysis. Antibodies/fluorescent conjugates were diluted to final working concentrations (as shown) in blocking buffer solution (see appendix) for; immunohistochemistry (IHC) or Fluorescent-Activated Cell Sorting (FACS). Antibodies for Western blot (WB) were diluted in TBS-T (see appendix). Blocking antibodies were diluted in differentiation medium (see appendix). 


\subsubsection{Transcript analysis}

\subsubsection{RNA extraction}

Total RNA was extracted from cells using the standard Trizol protocol (According to manufacturer's instructions). Using sterile filter pipette tips, pre-rinsed samples were carefully layered over with $1 \mathrm{ml}$ TRIzol ${ }^{\circledR}$ reagent per $10 \mathrm{~cm}^{2}$ culture dish (Ambion Life Technologies; 15596026). A cell scraper was used to collect the cell lysates. The resulting suspension was transferred into sterile $2 \mathrm{ml}$ microtubes and $200 \mu \mathrm{l}$ Chloroform (Applichem, A3691) was added to the tubes, mixed (vortex) and rested for 3 mins at RT. Samples were centrifuged at $12,000 \mathrm{~g}$ at $4{ }^{\circ} \mathrm{C}$ for $15 \mathrm{mins}$. The clear aqueous phase supernatant was carefully removed and transferred into RNase free 1.5 ml microfuge tubes. $500 \mu \mathrm{l}$ isopropanol (Roth, 6752.2) was added to the tubes, vortexed and rested for 10 mins to allow RNA precipitation. Next, samples were centrifuged at $12,000 \mathrm{~g}$ at $4{ }^{\circ} \mathrm{C}$ for 10 mins to pellet the RNA and then rinsed with $70 \% \mathrm{EtOH}$. Following another centrifuge step at $12,000 \mathrm{~g}$ at $4{ }^{\circ} \mathrm{C}$ for $5 \mathrm{mins}$, the RNA tubes were allowed to airdry for 10 mins. RNA samples were thereafter suspended in DEPC water (Sigma, 95284). The amount (ng/ml) and quality (260/280 nm OD. Ratio $\geq 1.8$ ) of RNA was finally assessed with a spectrophotometer (Nanodrop, ND-1000, ThermoScientific) at a wavelength of $260 \mathrm{~nm}$. Samples were subsequently stored at $80{ }^{\circ} \mathrm{C}$ until further use.

\subsubsection{RNA reverse transcription $(\mathrm{RT})$}

RNA extracted from samples was initially treated with DNase I (Roche, 04716728001) to remove contaminating genomic DNA. A reaction mixture containing $1 \mu \mathrm{g}$ RNA was treated with $0.2 \mu \mathrm{l}$ DNase I enzyme (10 U/ $\mu \mathrm{l})$, with the addition of $1 \mu \mathrm{l}$ DNase I buffer and incubated for 20 mins at $37{ }^{\circ} \mathrm{C}$. This was then followed by the addition of $1 \mu \mathrm{l}$ EDTA solution ( $40 \mu \mathrm{mol} / \mathrm{L}$ stock) and incubated for 10 mins at $75{ }^{\circ} \mathrm{C}$ to inactive DNase I activity. cDNA synthesis was subsequently performed on DNase treated samples using the High Capacity cDNA Reverse Transcription Kit (Applied Biosystems), following the manufacturer's instructions. A $2 \mathrm{X}$ mastermix was added (containing; $2 \mu 1$ 10X RT buffer, $0.8 \mu 125 \mathrm{X}$ dNTPs [100 mmol/L], $2 \mu 1$ 10X RT Random Primers, $1 \mu 1$ RNase inhibitor and $2.2 \mu 1$ DEPC water 
and $1 \mu \mathrm{l}$ Multiscribe Reverse Transcriptase) to each RNA sample. To control for genomic DNA contamination, a substitute reaction mixture containing $1 \mu$ l DEPC water (replacing $1 \mu 1$ Multiscribe Reverse Transcriptase) was additionally included for each sample [no reverse transcriptase (RT) control]. RNA samples were then place into the T gradient PCR thermocycler (Biometra) for PCR amplification $\left(25{ }^{\circ} \mathrm{C}\right.$ for 10 mins, $37^{\circ} \mathrm{C}$ for $120 \mathrm{mins}, 85^{\circ} \mathrm{C}$ for $5 \mathrm{mins}$, and then $4{ }^{\circ} \mathrm{C}$ ) for 40 cycles. The amplified cDNA products were subsequently diluted 1:5 in RNase-free water and stored at $-20{ }^{\circ} \mathrm{C}$ until further use.

\subsubsection{Polymerase chain reaction $(\mathrm{PCR})$}

PCR reactions were performed using the T gradient PCR thermocycler (Biometra) and Extaq DNA polymerase (TaKaRa Bio. Inc) according to manufacturer's instructions. A PCR (4X reaction) mixture contained: $37.7 \mu 1$ distilled water, $5 \mu 1$ 10X Ex Taq Buffer, $4 \mu \mathrm{l}$ dNTP mixture $(2.5 \mathrm{mM}), 1 \mu \mathrm{l}$ of each forward (F) and reverse (R) primers (10 mM), $0.25 \mu \mathrm{l} \mathrm{TaKaRa} \mathrm{Ex} \mathrm{Taq} \mathrm{(5} \mathrm{U/ \mu l)} \mathrm{and} 1 \mu \mathrm{l}$ cDNA (Section 2.3.2.2). The primer sequences used for PCR are listed in Table 4. The PCR conditions are described below in Table 3. After PCR amplification, the PCR products were premixed with suitable volumes of 6X DNA loading buffer (see appendix A2), loaded into $1.5 \%$ DNA agarose gels (see appendix A2) and separated by electrophoresis in $1 \mathrm{X}$ TAE gel running buffer (see appendix A2) at $90 \mathrm{~V}$ for approximately 30 mins. The band(s) of the respective DNA product sizes (Amplicons) were imaged by the Gel Doc XR (BioRad) system.

\begin{tabular}{|c|c|c|c|}
\hline Proceedure & Temperature $\left({ }^{\circ} \mathbf{C}\right)$ & Time & Cycles \\
\hline Initial denaturation & 98 & $30 \mathrm{sec}$ & 1 \\
\hline Denaturation & 98 & $10 \mathrm{sec}$ & \multirow{2}{*}{35} \\
\hline Annealing & 60 & $30 \mathrm{sec}$ & \\
\hline Elongation & 72 & $30 \mathrm{sec}$ & \\
\hline Final elongation & 72 & $5 \mathrm{mins}$ & 1 \\
\hline Stop & 4 & $\infty$ & 1 \\
\hline
\end{tabular}

Table 3. Thermocycler conditions used for PCR. 


\subsubsection{Quantitative polymerase chain reaction (qPCR)}

All qPCR runs were performed on the ABI PRISM 7900HT Fast Real-Time PCR system (Applied Biosystems) on 384-well plate format. A mastermix (9 $\mu \mathrm{l}$ ) was prepared for each different primer (targeting a specific gene of interest) containing: $3.75 \mu 1$ Nuclease-free water, $5 \mu 1$ Fast track SYBR ${ }^{\circledR}$ Green Master Mix (Applied Biosystems; 4385612) and $0.25 \mu$ l Primers (containing both forward (F) and reverse (R) primers; from $2 \mu \mathrm{M}$ stocks). All sample reactions were performed in triplicates. Mastermixes for each gene $(9 \mu \mathrm{l} /$ well) were firstly pipetted into a MicroAmp® Optical 384-Well Reaction Plate (Applied Biosystems, 4309849) on ice, followed by the addition of $1 \mu \mathrm{l}$ cDNA to each different mastermix respectively. Separate wells both containing $1 \mu \mathrm{l}$ 'no RT' and $1 \mu \mathrm{l}$ 'water' (no template DNA) were included as controls to ensure no genomic DNA presence or any primer contamination. Next, the 384-well plate was covered with a clear adhesive film (Applied Biosystems, 4306311) and centrifuged at 1,000 $\mathrm{g}$ for 2 mins. qPCR amplification was then performed with the following PCR conditions: $50{ }^{\circ} \mathrm{C}$ for 2 mins, denaturation at $95{ }^{\circ} \mathrm{C}$ for 10 mins and a cycle amplification at $95{ }^{\circ} \mathrm{C}$ for $15 \mathrm{~s}$ and $60{ }^{\circ} \mathrm{C}$ for $1 \mathrm{~min}$ (for a total of 40 cycles). A melting point dissociation curve step was added to determine primer specificity. The results obtained from the run were assessed on the Sequence Detection System ABI 7900HT v2.4 software (Applied Biosystems). The relative gene transcript expression, relative to the housekeeping gene expression in samples was analysed using the $\Delta \mathrm{Ct}$ or $\Delta \Delta \mathrm{Ct}$ method (Livak and Schmittgen, 2001). Cycle threshold $(\mathrm{Ct})$ values for all genes of interest were normalized to glyceraldehyde 3phosphate dehydrogenase (GAPDH) as a housekeeping gene. All primer sequences (F and R) are listed below (Table 4). GAPDH expression levels did not differ significantly between the investigated samples groups in all experiments, and was thus used for normalization. 


\begin{tabular}{|c|c|c|c|c|}
\hline $\begin{array}{c}\text { Gene } \\
\text { transcripts }\end{array}$ & 5' Forward primer & 3' Reverse primer & Ensembl code & $\begin{array}{l}\text { Base } \\
\text { Pairs }\end{array}$ \\
\hline Col1a1 & $\begin{array}{l}\text { ACC GAT GGA TTC } \\
\text { CCG TTC G }\end{array}$ & $\begin{array}{l}\text { GCT ACG CTG TTC TTG } \\
\text { CAG TG }\end{array}$ & ENSMUSG00000001506 & 134 \\
\hline Col1a2 & $\begin{array}{l}\text { GCT GGG AAA CAT } \\
\text { GGA AAC CG }\end{array}$ & $\begin{array}{l}\text { GGA TGC CTT GAG } \\
\text { GAC CAC TA }\end{array}$ & ENSMUSG00000029661 & 100 \\
\hline $\operatorname{Itg} \alpha 1$ & $\begin{array}{l}\text { CAA TGC CAG AAC } \\
\text { TTC GGC TG }\end{array}$ & $\begin{array}{l}\text { AAA GGG TCC TCA } \\
\text { AGG CTT CG }\end{array}$ & ENSMUSG00000042284 & 130 \\
\hline $\operatorname{Itg} \alpha 2$ & $\begin{array}{l}\text { GGG ACC TCA CAA } \\
\text { ACA CCT TCA G }\end{array}$ & $\begin{array}{l}\text { TCG CCA TCG GTC } \\
\text { ACA ACT AC }\end{array}$ & ENSMUSG00000015533 & 115 \\
\hline $\operatorname{Itg} \beta 1$ & $\begin{array}{l}\text { GCC AAA TCT TGC } \\
\text { GGA GAA TGT }\end{array}$ & $\begin{array}{l}\text { ACA TCG TGC AGA } \\
\text { AGT AGG CA }\end{array}$ & ENSMUSG00000025809 & 99 \\
\hline Hsp47 & $\begin{array}{l}\text { CTG CAG TCC ATC } \\
\text { AAC GAG TGG GC }\end{array}$ & $\begin{array}{l}\text { ATG GCG ACA GCC } \\
\text { TTC TTC TGC }\end{array}$ & ENSMUSG00000070436 & 403 \\
\hline Gapdh & $\begin{array}{l}\text { ATG TTC CAG TAT } \\
\text { GAC TCC ACT CAC G }\end{array}$ & $\begin{array}{l}\text { GAA GAC ACC AGT } \\
\text { AGA CTC CAC GAC A }\end{array}$ & ENSMUSG00000057666 & 171 \\
\hline$\beta$-Actin & $\begin{array}{l}\text { CTA AGG CCA ACC } \\
\text { GTG AAA AGA }\end{array}$ & $\begin{array}{l}\text { AGA GGC ATA CAG } \\
\text { GGA CAG CA }\end{array}$ & ENSMUSG00000029580 & 101 \\
\hline $\begin{array}{l}\text { SERPINH1 } \\
\text { (Exon 3) }\end{array}$ & $\begin{array}{l}\text { CAG CGC CTT CTG CCT } \\
\text { CCT GGA G }\end{array}$ & $\begin{array}{l}\text { CGC CTT GCC GCC } \\
\text { CAG CGA CAC }\end{array}$ & ENSG00000149257 & 241 \\
\hline $\begin{array}{l}\text { SERPINH1 } \\
\text { (Exon 4-5) }\end{array}$ & $\begin{array}{l}\text { CCG TGG GTG TCA } \\
\text { TGA TGA TG }\end{array}$ & $\begin{array}{l}\text { CTT CCC CAT CCA GAT } \\
\text { CTT CAG C }\end{array}$ & ENSG00000149257 & 197 \\
\hline $\begin{array}{l}\text { SERPINH1 } \\
\text { (Exon 5-6) }\end{array}$ & $\begin{array}{l}\text { GAA AAG CTG CTA } \\
\text { ACC AAA G }\end{array}$ & $\begin{array}{l}\text { GAA GAT GAA GGG } \\
\text { GTG GTC G }\end{array}$ & ENSG00000149257 & 327 \\
\hline GAPDH & $\begin{array}{l}\text { CCT CAA GAT CAT } \\
\text { CAG CAA TGC C }\end{array}$ & $\begin{array}{l}\text { ATG TTC TGG AGA } \\
\text { GCC CCG C }\end{array}$ & ENSG00000111640 & 189 \\
\hline
\end{tabular}

Table 4. Primers used in RT-PCR and qPCR analysis

\subsubsection{RNA sequencing (RNA-seq) and bioinformatics}

RNA-seq analysis was performed at the Transcriptome and Genome Analysis Laboratory at the University Medical Center Göttingen. Total RNA was isolated from samples (hES2-CMs, hcFBs and hFFBs; $\mathrm{n}=3$ /group) using the Trizol extraction method, as described in Section 2.3.2.1. RNA quality was assessed with the Bioanalyzer 2100 Fragment Analyzer and all samples subjected to RNA sequencing exhibited an RNA integrity number of $>7$. Total RNA was thereafter subjected to library preparation (TruSeq Stranded Total RNA Sample Prep Kit from Illumina) and RNA-sequencing was performed on an Illumina HighSeq-2000 platform (50 bp single end run; >25 million reads /sample). 
Sequence images were transformed with Illumina software BaseCaller to bcl files, which were demultiplexed to fastq files with CASAVA (v1.8.2). Reads were aligned by STAR (2.3.9e) to the Ensembl human genome (37.74) (Dobin et al., 2013). Counting the reads to each gene to the Ensembl gene annotation file gtf (37.74) was done via HTSeq python scripts. Data was pre-processed and analysed in the $\mathrm{R}$ environment (3.0.2/2.14) loading DESeq, gplots, and biomaRt packages (Durinck et al., 2009). After filtering the genes exceeding more than 20 counts for at least one sample; normalisation, estimation of dispersions and testing for differentially expressed genes based on a test assuming negative binomial data distribution was computed via DESeq (Anders and Huber, 2010). Candidate genes were filtered to a minimum of $2 \mathrm{x}$ fold change and False Discovery Rate (FDR) corrected to $\mathrm{P}<0.05$. Functional association for the candidates genes were analysed using gene ontology and Kyoto Encyclopedia of Genes and Genomes pathway enrichment via goseq.

The script language $\mathrm{R}$ was utilized for data analysis and for heat map generation, together with the gplot-package for visualizing the expression values for pre-selected genes from the normalized RNA-seq data (Development Core Team R, 2009; Warnes et al., 2009).

\subsection{Assessment of integrin-ECM signalling in EHM formation}

\subsubsection{Integrin blocking in EHM formation}

To investigate the role of collagen binding integrin Itg $\alpha 1, \operatorname{Itg} \alpha 2$ and $\operatorname{Itg} \beta 1$ ) signalling in EHM formation, human and mouse purified CM and FB populations were mixed (75\% purified CMs $+25 \%$ FBs) and incubated with the respective integrin blocking antibodies targeting integrin $\alpha 1$ chains (CD49a; $50 \mu \mathrm{g} / \mathrm{ml}$ ), integrin $\alpha 2$ chains (CD49b; $50 \mu \mathrm{g} / \mathrm{ml}$ ) and Integrin $\beta 1$ chains (CD29; $40 \mu \mathrm{g} / \mathrm{ml}$ ) (Table 2) at $37{ }^{\circ} \mathrm{C}$ for 30 minutes. As EHMs failed to form condensed tissue in the absence of FBs, EHMs generated with purified CMs (only) was used a negative control for tissue contraction. EHMs were generated (as described in Section 2.2.1) and $40 \mu \mathrm{l}$ of EHM reconstitution mixture were distributed into wells $\left(1.25 \times 10^{5}\right.$ cells/well; scaled down to 1:12 of the original master mix for $\mathrm{x} 1 \mathrm{EHM}$ ) of an ultra-low attachment surface flat 
bottomed 96-microwell plate (Corning, 13414054) and incubated at $37{ }^{\circ} \mathrm{C}$ in a humidified incubator with $5 \% \mathrm{CO}_{2}$. Surface treated ultra-low attachment plates prevented the EHM reconstitution mixtures from sticking to the sides/bottom of wells.

After $1 \mathrm{hr}$ of initial casting, $100 \mu \mathrm{l}$ of fresh differentiation medium supplemented with the respective; $\alpha 1$ chain $(50 \mu \mathrm{g} / \mathrm{ml}), \alpha 2$ chain $(50 \mu \mathrm{g} / \mathrm{ml})$ and $\beta 1$ chain $(40 \mu \mathrm{g} / \mathrm{ml})$ blocking antibodies (as described above) were added to wells and placed back into culture. After 21 hrs of casting ('blocking'), images of EHMs in wells were captured using a high throughput automated Cellavista machine (SynenTec Bio Services $\mathrm{GmbH})$. The medium in each well was then carefully removed using a microwell aspirator, without making contact with the tissues and replaced with $100 \mu \mathrm{l}$ of fresh differentiation medium to wash out the blocking antibody from tissues. After $72 \mathrm{hrs}$ of casting ('recovery'), images of EHMs in wells were again obtained. The surface area of retracted EHM tissues during blocking ( $21 \mathrm{hrs}$ ) and recovery (72 hrs) was assessed using Image $\mathbf{J}$ software (National Institutes of Health). The percentage (\%) of EHM contraction/retraction per well was calculated as summarized below:

$100-\left(\frac{\text { EHM surface area }\left(\mathrm{mm}^{2}\right)}{\text { Total well surface area }\left(\mathrm{mm}^{2}\right)} \times 100\right)=$ EHM well contraction (\%)

\subsection{Assessment of collagen processing heat shock protein 47 knockout (HSP47 KO) MEFs}

\subsubsection{HSP47 KO MEFs}

HSP47 KO MEFs were kindly provided from the lab of Prof. Nagata (Kyoto Sangyo University, Kyoto, Japan). The HSP47 KO MEF cell line was established from 8.5 dpc embryos of HSP47 KO mice (C57BL/6J), in which the Hsp47 gene was disrupted using homologous recombination with targeting vectors deleting exon 4 and a part of exon 5 (Nagai et al., 2001). These collagen processing-defective HSP47 KO MEFs were compared to wild-type (WT) C57BL/6J MEFs for all purposes of this study. 


\subsubsection{Characterization of HSP47 KO MEFs}

The absence of HSP47 expression in HSP47 KO MEFs in culture, was characterized at both RNA level via RT-PCR (Section 2.3.2.2 and 2.3.2.3), and at protein level by immunoblotting analysis (Section 2.3.1.4) and immunoflourescence staining (Section 2.7.4), respectively.

\subsection{Investigating collagen secretion in HSP47 KO MEFs}

\subsubsection{Assessment of soluble collagen secretion}

To quantitatively compare the amount of soluble collagen secreted from WT and HSP47 KO FBs, the amount of soluble collagen in the culture medium was assessed using the soluble Sircol ${ }^{\mathrm{TM}}$ assay kit. A schematic outline of the experiment is summarized in Figure 11:

Low Serum Medium

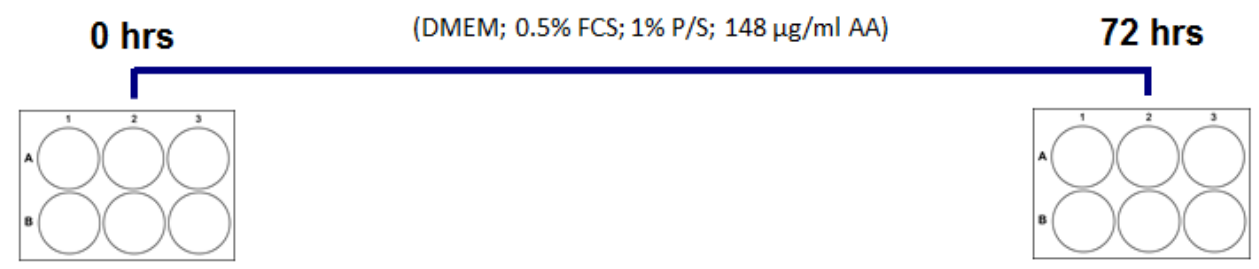

Seed WT or HSP47 KO FBs

( $3 \times 10^{5}$ cells/well)
1) Conditioned medium analysis (Sircol ${ }^{\mathrm{TM}}$ )

2) Cell number (Cellavista)

Figure 11. The measurement of soluble collagen secretion in MEFs. WT and HSP47 KO MEFs were resuspended in low serum medium (containing; DMEM, 0.5\% FCS; $1 \% \mathrm{P} / \mathrm{S} ; 148 \mu \mathrm{g} / \mathrm{ml} \mathrm{L-}$ Ascorbic Acid 2-phosphate magnesium salt [AA]) and seeded on 6 well plates ( 3 x 10 $10^{5}$ cells/well; 2 $\mathrm{ml} /$ well). Cells were cultured for $72 \mathrm{hrs}$ in at $37{ }^{\circ} \mathrm{C}, 5 \% \mathrm{CO}_{2}$. Conditioned medium and cells were thereafter subjected to 1) conditioned medium analysis using the Sircol ${ }^{\mathrm{TM}}$ assay kit, and 2) cells were counted using the Cellavista image platform (SynenTec Bio Services GmbH), respectively.

The cell conditioned medium from each well was transferred into fresh $2 \mathrm{ml}$ microtubes and pelleted at $300 \mathrm{x} \mathrm{g}$ for $5 \mathrm{mins}$ at RT, to remove cellular debris. Conditioned medium $(1 \mathrm{ml})$ was then transferred into clean $1.5 \mathrm{ml}$ microtubes and 
total soluble collagen in the conditioned medium was measured using the Sircol ${ }^{\mathrm{TM}}$ assay kit (Biocolor), according to manufacturer's instructions. Low serum medium (1 $\mathrm{ml}$ ) cultivated on plates in the absence of cells were used as normalization controls. Conditioned medium samples were initially treated with $200 \mu \mathrm{l}$ ice-cold Isolation and Concentration reagent (provided by the manufacturer's), mixed and incubated overnight at $4{ }^{\circ} \mathrm{C}$ to concentrate the collagen within samples, prior to measurements.

Next, samples were centrifuged at $15,300 \mathrm{x} g$ and supernatants were then carefully removed leaving a remaining $100 \mu \mathrm{l}$ and the invisible pellet of hydrated transparent collagen at the bottom of the microtube. Rat tail collagen reference standards $(0,1.25$, 2.5, 5, $10 \mu \mathrm{g}$; provided by the manufacturer) were then prepared in $100 \mu \mathrm{l}$ sterile water. Sircol dye reagent $(1 \mathrm{ml})$ containing Sirius red in picric acid was added to each standard, control and sample tubes. Microtubes were then mixed by inversion and placed on a mechanical rocking platform (BioMetra) for 30 mins at RT in order to form collagen-dye complexes which precipitate out collagen from the soluble unbound dye. Tubes were then centrifuged again at $15,300 \mathrm{x}$ g for $10 \mathrm{mins}$ at $4{ }^{\circ} \mathrm{C}$, and carefully inverted to remove the excess unbound dye reagent. Provided ice-cold Acid-Salt wash $(750 \mu \mathrm{l})$ was gently added to tubes and gently mixed in order to remove any unbound dye on the surface of pellets and on the inside surfaces of tubes. Microtubes were again centrifuged at $15,300 \mathrm{x}$ g for $10 \mathrm{mins}$ at $4{ }^{\circ} \mathrm{C}$ and acid-wash reagent was gently drained out from the tubes as much as possible without disturbing the red/pink precipitated collagen-dye pellet. To release and remove collagen from the bound dye, $250 \mu \mathrm{l}$ of provided ice-cold Alkali reagent was added to all tubes. Microtubes were mixed until the bound dye dissolved ( $\sim \mathrm{mins})$, and were subsequently transferred into individual wells $(200 \mu \mathrm{l})$ of a 96-microwell plate.

The absorbance of standards, controls and samples at $555 \mathrm{~nm}$ was detected with the Flexstation ${ }^{\circledR} 3$ Multi-mode microplate reader, against water (blank) and the collagen amount of all conditioned medium samples (in $2 \mathrm{ml}$ ) were calculated from standard curves. The measured value of collagen in control tubes (containing low serum medium only) was subtracted from the sample values.

Plates used in the experiment outlined above were then washed once in 1X PBS and subsequently fixed in Roti $^{\circledR}$ Histofix $4 \%$ for 15 mins at RT. Cells were then washed 
(x2) in 1X PBS, permeabilized in blocking buffer solution (see appendix A2), and labelled with Hoechst. Thereafter, the total number of fluorescence-labelled cell nuclei in each well was assessed using a high-throughput automated imaging platform (Cellavista; SynenTec Bio Services GmbH). The total amount of soluble collagen normalized to cell number $(x \mathrm{~g} / \mathrm{cell})$ was thus determined from these two experiments, as calculated below: $\frac{\text { Total collagen released into medium/well }(\mathrm{xg})^{*}}{\text { Total cell nuclei/well (cells) }}=$ Total soluble Collagen/cell $[\mathrm{xg} / \mathrm{cell}]$

* Determined via Soluble Collagen Sircol ${ }^{\mathrm{TM}}$ assay kit.

\# Determined via Cell nuclei counting/Cellavista.

\subsubsection{Deoxychelate (DOC)-separation ECM components}

WT and HSP47KO MEFs were assessed for differences in ECM secretion. The protocol for extraction of the deoxycholate (DOC)-insoluble ECM in MEFs was modified from (Van Duyn Graham et al., 2010; Midwood et al., 2002). WT and HSP47 KO MEFs were cultured at high seeding density $\left(1 \times 10^{5}\right.$ cells/well, on 24well plates) for 7 days in MEF medium containing: $148 \mu \mathrm{g} / \mathrm{ml}$ L-Ascorbic Acid 2phosphate magnesium salt. Culture medium was removed and cells were washed once in 1X PBS. Cells and ECM suspensions were harvested in $300 \mu$ l cold DOC buffer (4 $\%$ DOC in 20 mM Tris-HCl, pH 8.8 and Protease inhibitors [Roche]), scraped off and transferred into $1.5 \mathrm{ml}$ microtubes. The extracts were homogenized (x5) with a $27 \frac{1}{2}$ gauge needle and then pelleted at $18,000 \mathrm{x}$ g for $20 \mathrm{mins}$ at $4{ }^{\circ} \mathrm{C}$. The supernatants (DOC-soluble fraction) were carefully collected and transferred into fresh $1.5 \mathrm{ml}$ microtubes. The pellets were washed again with $200 \mu 1$ DOC buffer and centrifuged at $18,000 \mathrm{x}$ g for $20 \mathrm{mins}$ at $4{ }^{\circ} \mathrm{C}$. The supernatant was transferred into the DOC-soluble fraction, containing cellular material and components not incorporated into the ECM. The DOC-insoluble pellet fraction containing ECM components (Van Duyn Graham et al., 2010; Midwood et al., 2002) was solubilised in $200 \mu 1$ DOC insoluble buffer (1\% SDS, $25 \mathrm{mmol} / \mathrm{l}$ Tris-HCl, $\mathrm{pH}$ 8.0, and protease inhibitors). DOC-insoluble ECM fractions were incubated at $95{ }^{\circ} \mathrm{C}$ for 5 mins. 
Prior to SDS-PAGE analysis, protein content was quantified with a standard Pierce $^{\mathrm{TM}}$ bicinchoninic acid (BCA) protein assay (ThermoFisher Scientific), to standardize loading protein concentrations (Smith et al., 1985). Proteins were resolved on 10\% SDS-polyacrylamide gels (as described in Section 2.3.1.4). A rat tail collagen sample was used as a comparative molecular weight reference for collagen type I. To visualize all proteins resolved on gels from DOC insoluble ECM fractioned samples, coomassie blue staining was used to stain SDS-PAGE gels: Resolved SDS-PAGE gels were carefully removed from casting chambers and were gently washed (x3) in deionized water for 5 mins at RT. Gels were then incubated for $1 \mathrm{hr}$ at RT in Coomassie Brilliant Blue solution (G-250). Next, staining solution was carefully removed and SDS-PAGE gels were washed once in distilled water for 5 mins to remove any unbound dye, and then rinsed overnight at RT. Images of coomassie stained gels were taken on the ChemDoc ${ }^{\mathrm{TM}}$ MP Imaging System (BioRad).

\subsubsection{Mass spectrometric analysis of DOC-insoluble ECM components}

Mass spectrometric (MS) analyses were performed by the Proteomics Core Facility at the University Medical Center Göttingen (Dr. C. Lenz; Prof. H. Urlaub).

\subsubsection{Sample preparation}

DOC-insoluble ECM fractions from WT- and HSP47 KO MEFs (n=1 sample/group) were reconstituted in $1 \times$ NuPAGE LDS Sample Buffer (Invitrogen) and separated on 4-12\% NuPAGE Novex Bis-Tris Minigels (Invitrogen). Gels were stained with Coomassie Blue for visualization purposes, and each lane was sliced into 23 equidistant segments, regardless of staining. After washing, gel slices were reduced with dithiothreitol (DTT), alkylated with 2-iodoacetamide and digested with trypsin overnight. The resulting peptide mixtures were then extracted, dried in a SpeedVac, reconstituted in $2 \%$ acetonitrile/ $0.1 \%$ formic acid/ (v:v) and prepared for Nanoscale liquid chromatography coupled to tandem mass spectrometry (nanoLC-MS/MS) as described previously (Atanassov and Urlaub, 2013). 


\subsubsection{NanoLC-MS/MS analysis}

For MS analysis, samples were enriched on a self-packed reversed phase-C18 precolumn (0.15 mm ID x $20 \mathrm{~mm}$, Reprosil-Pur120 C18-AQ $5 \mu \mathrm{m}$, Dr. Maisch, Ammerbuch-Entringen, Germany) and separated on an analytical reversed phase-C18 column (0.075 mm ID x 200 mm, Reprosil-Pur 120 C18-AQ, $3 \mu \mathrm{m}$, Dr. Maisch) using a 30 min linear gradient of 5-35\% acetonitrile/ $0.1 \%$ formic acid (v:v) at $300 \mathrm{nl}$ $\min ^{-1}$ ). The eluent was analyzed on a $\mathrm{Q}$ Exactive hybrid quadrupole/orbitrap mass spectrometer (ThermoFisher Scientific, Dreieich, Germany) equipped with a FlexIon nanoSpray source and operated under Excalibur 2.4 software using a data-dependent acquisition method. Each experimental cycle was of the following form: one full MS scan across the 350-1600 mass-to-charge ratio $(\mathrm{m} / \mathrm{z})$ range was acquired at a resolution setting of 70,000 Full Width at Half Maximum (FWHM), and Automatic Gain Control (AGC) target of $1 \times 10^{6}$ and a maximum fill time of $60 \mathrm{~ms}$. Up to the 12 most abundant peptide precursors of charge states 2 to 5 above a $2 \times 10^{4}$ intensity threshold were then sequentially isolated at 2.0 FWHM isolation width, fragmented with nitrogen at a normalized collision energy setting of $25 \%$, and the resulting product ion spectra recorded at a resolution setting of 17,500 FWHM, and AGC target of $2 \times 10^{5}$ and a maximum fill time of $60 \mathrm{~ms}$. Selected precursor $\mathrm{m} / \mathrm{z}$ values were then excluded for the following $15 \mathrm{~s}$. Two technical replicates per sample were acquired.

\subsubsection{Data processing}

Peaklists were extracted from the raw data using Raw2MSMS software v1.17 (Max Planck Institute for Biochemistry, Martinsried, Germany). Protein identification was achieved using MASCOT 2.4 software (Matrixscience, London, United Kingdom). Proteins were identified against the UniProtKB mouse reference proteome v2014.12, along with a set of 51 contaminants commonly identified in our laboratory. The search was performed with trypsin as enzyme and iodoacetamide as cysteine blocking agent. Up to two missed tryptic cleavages and methionine oxidation as a variable modification were allowed for. Search tolerances were set to $10 \mathrm{ppm}$ for the precursor mass and 0.05 Da for fragment masses, and ESI-QUAD-TOF specified as the instrument type. 
Scaffold software version 4.4.1.1 (Proteome Software Inc., Portland, OR) was used to validate MS/MS based peptide and protein identifications. Peptide identifications were accepted if they could be established at greater than $95.0 \%$ probability by the Percolator algorithm. Protein probabilities were assigned by the Protein Prophet algorithm (Nesvizhskii et al., 2003). Protein identifications were accepted if they could be established at greater than $99 \%$ by the Percolator algorithm and contained at least 2 identified peptides. Protein hits that contained similar peptides and could not be differentiated based on MS/MS analysis alone were grouped to satisfy the principles of parsimony. Proteins sharing significant peptide evidence were grouped into clusters. Proteins were annotated with GO terms from NCBI downloaded Feb 23, 2015 (Ashburner et al, 2000). Relative quantification of proteins in the samples was achieved by Analysis of Variance (ANOVA) of normalized Spectral Counts using a Benjamini-Hochberg-corrected $\mathrm{p}$ value of 0.05 to judge significance. To allow for the calculation of low abundance protein ratios, a minimum value of 3 spectral counts was introduced where necessary.

\subsection{Fibroblast-derived ECM characterization}

\subsubsection{Generation of fibroblast-derived ECM}

FB-derived ECM was generated as previously described with minor modifications (Beacham et al., 2007). WT or HSP47 KO MEFs were plated at high seeding density on 24-well tissue culture plates and cultured for 11 days to deposit matrices on precoated glass coverslips. Firstly, sterile $15 \mathrm{~mm}$ glass coverslips (prior to cell seeding) were incubated in $0.2 \%$ prepared gelatine solution (Sigma, G2500) for $1 \mathrm{hr}$ at $37{ }^{\circ} \mathrm{C}$. Coverslips were then washed once in 1X PBS and thereafter treated with $1 \%$ Gluteraldehyde (Sigma-Aldrich, G5882; prediluted in 1X PBS and sterile filtered through a $0.22 \mu \mathrm{m}$ syringe filter) for $30 \mathrm{mins}$ at RT to crosslink Gelatine and stabilize the attachment of ECM to culture dish surfaces. Coverslips were subsequently thoroughly washed $(\mathrm{x} 3)$ in $1 \mathrm{X}$ PBS and treated with $1 \mathrm{~mol} / \mathrm{L}$ ethanolamine (SigmaAldrich, E9508; prediluted in deionized water and sterile filtered through a $0.22 \mu \mathrm{m}$ syringe filter) for 30 mins and RT. Coverslips were again thoroughly washed (x3) with $1 \mathrm{X}$ PBS and ready for use. 
MEFs (WT and HSP47 KO) were subsequently plated at high seeding density (1x $10^{5}$ cells/well) onto coated coverslips. After $24 \mathrm{hrs}$ of cell seeding, medium was replaced with fresh MEF medium containing; $148 \mu \mathrm{g} / \mathrm{ml}$ L-Ascorbic Acid 2-phosphate magnesium salt (Sigma-Aldrich), to facilitate in the deposition of a dense ECM network (Beacham et al., 2007). Medium was changed every second day. Decellurization was performed by utilizing prewarmed extraction buffer containing: $0.5 \%$ Triton X-100 (Sigma, T8787); $20 \mathrm{mmol} / \mathrm{l}$ mmonium hydroxide $\left(\mathrm{NH}_{4} \mathrm{OH}\right.$; Sigma-Aldrich, 338818) in 1X PBS. Plates were incubated for approximately 2-5 mins at $37{ }^{\circ} \mathrm{C}$ and $5 \% \mathrm{CO}_{2}$, until no intact cells are visible under the inverted microscope. 1X PBS was gently added and plates were stored overnight at $4{ }^{\circ} \mathrm{C}$. Afterwards, cellular debris was carefully aspirated to prevent detachment of the freshly denuded ECM layer. Plates were gently washed once in $1 \mathrm{X}$ PBS and incubated with DNase I $(10 \mathrm{U} / \mathrm{ml})$ for $30 \mathrm{mins}$ at $37{ }^{\circ} \mathrm{C}$. Matrices were thereafter very carefully washed (x2) in $1 \mathrm{X}$ PBS and stored at $4{ }^{\circ} \mathrm{C}$ until further use. Complete decellurization was assessed by Hoechst and F-Actin staining (Section 2.7.4). The quality of the FB-derived ECMs was assessed with Ponceau S staining (Section 2.7.2), under an inverted phase-contrast microscope. Prior to the seeding of enriched mESC-CMs, the FB-derived ECMs were incubated with 5\% FBS (prediluted in 1X PBS) for $1 \mathrm{hr}$ at $37^{\circ} \mathrm{C}$.

\subsubsection{Ponceau S staining}

PBS was gently aspirated from decellularized ECM. Plates were then incubated with $0.5 \mathrm{ml}$ Ponceau S solution (Sigma Aldrich; P7170) for 15 mins at RT. After staining, Ponceau S solution was carefully aspirated and plates were was washed once with 1 $\mathrm{ml}$ deionized water. The decellurized ECMs became visible and look as a fibrous mesh-like network of amorphous material. Brightfield imaging was performed with a Zeiss AxioObserver.A1 inverted microscope equipped with an AxioCam MRc5 camera (Zeiss). 


\subsubsection{Seeding of purified mESC-cardiomyocytes on fibroblast-derived ECMs}

To investigate the cardio-instructive properties of FB-derived ECM, purified mESCCMs were seeded on 24-well plates $\left(3 \times 10^{4}\right.$ cells/well) and cultured on either pretreated ( $0.2 \%$ gelatine) coverslips (as described in section 2.7.1) or FB-derived ECMs for a total of 6 days. Medium was replaced with fresh Differentiation medium every $48 \mathrm{hrs}$.

\subsubsection{Immunoflourescence imaging}

Samples were initially fixed in Roti® Histofix $4 \%$ for 15 mins at RT, followed by one wash in 1X PBS. Next, samples were blocked and permeabilized with blocking buffer solution (see appendix A2) for 30 mins, and were subsequently incubated with the respective primary antibodies (Table 2) for 60 mins at RT. Samples were then washed (x2) in blocking buffer for 3 min intervals, followed by secondary antibody incubation with respective antibodies/dyes (Table 2) for 60 mins in the dark at RT. Samples were subsequently washed in blocking buffer (x2) after each incubation step and thereafter stored in PBS. Coverslip-containing samples were removed from plates and mounted into microscope slides. Microscope slides were imaged using either the Olympus inverted fluorescence microscope camera (XM-10) or the Zeiss LSM710 confocal microscope.

\subsubsection{Image analysis}

Image $\mathbf{J}$ software (National Institutes of Health) was used to determine from images the; aspect ratio (AR), roundness, mean area $\left(\mu \mathrm{m}^{2}\right)$ and mean length $(\mu \mathrm{m})$ of CMs on cardio-instructive FB-derived ECM (Section 2.7.3). Cell area and AR was determined by measuring the $\alpha$-actinin fluorescence staining of the cells. Cell clumps with $>2$ DAPI stained nuclei were excluded from the analysis. 


\subsection{Assessment of HSP47 KO MEFs on EHM formation and functionality.}

The influence of HSP47 KO MEFs on EHM formation and functionality were performed, with methods described in Sections 2.2 (EHM construction [2.2.1], force of contraction [2.2.2] and whole mount EHM immunostaining [2.2.3]).

\subsection{Generation of human HSP47 mutant cell lines}

To investigate the influence of collagen processing HSP47 in the human system, the CRISPR (Clustered Regularly Interspaced Short Palindromic Repeats)/ CRISPR associated protein 9 (Cas9) nickase system was used for genome editing by introducing site-specific mutations in the SERPINH1 gene in hES2-RFP cells. Two SERPINH1 mutant hES2 lines were generated by Dr. Claudia Noack (Institute of Pharmacology, University Medical Center Göttingen). The first mutant line (Mut.1) was generated based on a recent mutation identified in a human patient (Christiansen et al., 2010) by CRISPR/Cas9 mediated deletion in exon 3 of the SERPINH1 locus. The second mutant line (Mut.2) was generated simulating the KO in the HSP47 mouse model (Nagai et al., 2000) by mediated deletion in exons 4-5.

In brief, guide RNA (gRNA) sequences were designed to target site specific 20 nucleotide sequences upstream of a NGG protospacer adjacent motif (PAM). This guides the Cas9 enzyme to its specific complementary target DNA sequence, facilitating a site-specific cleavage of the DNA. To minimize off-target cleavage, a double-nicking strategy was used via the Cas9 nickase mutant Cas9 (D10A) by cloning the gRNA sequences into the pX335A-hCas9 (D10A) vector (Kindly provided by Dr. Greber, MPI Münster). These CRISPR gRNA-Cas9n plasmids were thereafter transfected into pluripotent hES2-RFP cells. This strategy led only to single strand breaks of genomic DNA. After nicking of both DNA strands, the genomic region were repaired by the non-homologous end joining (NHEJ) process, leading to insertion/deletion mutations. NHEJ can mediate gene knock-outs for example via frameshift mutations with introduction of premature stop codons; in frame mutations will result in a modified protein product with changed/impaired function. After 
transfection, clones were selected and genotyping was performed to screen for positive SERPINH1 mutant clones (Section 2.9.1). The positive clones for Mut.1 and Mut.2 were subsequently grown and further characterized at RNA and protein level (Section 2.9.2).

\subsubsection{Genotyping}

Positive clones were validated by genotyping. Experiments were performed by Dr. Claudia Noack (Institute of Pharmacology, University Medical Center Göttingen). Genomic DNA was extracted from colonies (half) using the NucleoSpin ${ }^{\circledR}$ Tissue Kit (740952.250, Macherey-Nagel), according to manufacturer's instructions. The extracted DNA was used as a template for PCR. The primers for detecting the site specific SERPINH1 mutations are listed in Table 5.

\begin{tabular}{|l|l|l|c|c|}
\hline \multicolumn{1}{|c|}{ Gene } & 5' Forward primer & 3' Reverse primer & $\begin{array}{c}\text { Product size } \\
\text { (WT) }\end{array}$ & $\begin{array}{c}\text { Product size } \\
\text { (Mutation) }\end{array}$ \\
\hline $\begin{array}{l}\text { SERPINH1 } \\
\text { (Exon 3) }\end{array}$ & $\begin{array}{l}\text { CTT TGA GTA GGG AGG } \\
\text { TAA CAG AGG C }\end{array}$ & $\begin{array}{l}\text { CGC ACG AAG TCA TCA } \\
\text { GCG A }\end{array}$ & $663 \mathrm{bp}$ & Mut 1:502 bp \\
\hline $\begin{array}{l}\text { SERPINH1 } \\
\text { (Exon 4-5) }\end{array}$ & $\begin{array}{l}\text { TTA AGG GTG GTC ATG } \\
\text { GGG CA }\end{array}$ & $\begin{array}{l}\text { CAG ACC TGC TGA ATT } \\
\text { GGT GAG }\end{array}$ & $943 \mathrm{bp}$ & Mut 2: $762 \mathrm{bp}$ \\
\hline
\end{tabular}

Table 5. Primers for the genotyping of the SERPINH1 mutation hES2 cells.

For each PCR reaction: $1 \mu \mathrm{l}$ DNA, $4 \mu \mathrm{l}$ 5x GC-Buffer (1x), $0.4 \mu 1$ dNTPs (10 $\mathrm{mmol} / \mathrm{L}), 0.2 \mu \mathrm{l}$ of each primer (100 $\mu \mathrm{mol} / \mathrm{L}), 1 \mu \mathrm{l}$ DMSO (100\%), $0.2 \mu 1$ Phusion Polymerase and $13 \mu \mathrm{l} \mathrm{ddH}_{2} \mathrm{O}$ were mixed in a total volume of $20 \mu \mathrm{l}$. PCR was performed using the $\mathrm{T}$ gradient PCR thermocycler (Biometra). $\mathrm{H}_{2} \mathrm{O}$ was used as a negative control for genomic DNA. The PCR conditions used for each of the primers are outlined in Table 6. 


\begin{tabular}{|c|c|c|c|}
\hline \multicolumn{4}{|c|}{ SERPINH1 (Exon 3) primers } \\
\cline { 1 - 3 } Proceedure & $\begin{array}{c}\text { Temperature } \\
\left({ }^{\circ} \text { C) }\right.\end{array}$ & Time & Cycles \\
\hline $\begin{array}{c}\text { Initial } \\
\text { denaturation }\end{array}$ & 98 & $30 \mathrm{sec}$ & 1 \\
\hline Denaturation & 98 & $30 \mathrm{sec}$ & \multirow{2}{*}{28} \\
\cline { 1 - 3 } Annealing & 66 & $30 \mathrm{sec}$ & \multirow{2}{*}{1} \\
\hline Elongation & 72 & $30 \mathrm{sec}$ & \\
\hline $\begin{array}{c}\text { Final } \\
\text { elongation }\end{array}$ & 72 & $7 \mathrm{mins}$ & 1 \\
\hline Stop & 4 & $\infty$ & \\
\hline
\end{tabular}

\begin{tabular}{|c|c|c|c|}
\hline \multicolumn{4}{|c|}{ SERPINH1 (Exon 4-5) primers } \\
\hline Proceedure & $\begin{array}{c}\text { Temperature } \\
\left({ }^{\circ} \mathrm{C}\right)\end{array}$ & Time & Cycles \\
\hline $\begin{array}{c}\text { Initial } \\
\text { denaturation } \\
\end{array}$ & 98 & $30 \mathrm{sec}$ & 1 \\
\hline Denaturation & 98 & $30 \mathrm{sec}$ & \multirow{3}{*}{30} \\
\hline Annealing & 66 & $30 \mathrm{sec}$ & \\
\hline Elongation & 72 & $30 \mathrm{sec}$ & \\
\hline $\begin{array}{c}\text { Final } \\
\text { elongation }\end{array}$ & 72 & 7 mins & 1 \\
\hline Stop & 4 & $\infty$ & 1 \\
\hline
\end{tabular}

Table 6. Thermocycler conditions used for genotype PCR.

The PCR products were checked with agarose electrophoresis to screen for positive hES2 mutant colonies, under UV visualization with the Gel Doc XR (BioRad) system. The DNA product sizes of Mut. 1 (Exon 3 mutation) and Mut.2 (Exon 4-5 mutation) clones were smaller in size (for the respective primer) compared to WT, indicating that the CRISPR/Cas9 system genome editing was successful in mutating these site specific regions. The correct DNA fragments from these positive clones were subsequently excised from gels and DNA-gel extraction clean-up was performed using the NucleoSpin ${ }^{\circledR}$ Gel and PCR Clean-up Kit (740609.250, Macherey-Nagel), according to manufacturer's instructions. DNA sequencing was performed on the excised PCR products with the primers used for PCR (Table 5), to verify the mutations.

\subsubsection{Characterization of SERPINH1 mutant hES2 lines}

The expression of HSP47 at both mRNA and protein level was characterized on the positive SERPINH1 mutant hES2 lines (Mut.1 and Mut. 2) via RT-PCR (Section 2.3.2), immunoblotting (Section 2.3.1) and immunohistochemisty (Section 2.7.4). 


\subsection{Statistical analysis}

Data are presented as mean \pm SEM. Statistical analysis was performed using 1-way ANOVA followed by the Bonferroni's multiple comparison test, when comparing more than 2 groups. Alternatively, pair wise group comparisons were performed using two-tailed Student's unpaired t-test (Gaussian distribution was assumed). A value of * $\mathrm{P}<0.05$ was considered statistically significant. Data analysis was performed with GraphPad Prism v5.01 (GraphPad Software Inc., San Diego). 


\section{Results}

\subsection{EHM as a defined model of heart muscle development}

\subsubsection{Cardiomyocyte differentiation and purification}

The heart is a complex multicellular organ comprised of CMs and non-myocytes. Several lines of evidence have suggested that the non-myocytes, particularly cFBs are important in mediating CM growth and cardiac tissue formation. Initial studies in our lab utilized the EHM rodent model of heart muscle development to investigate whether cFBs are able to promote cardiac tissue formation and maturation (Naito et al., 2006; Christalla, Dissertation 2011). Specifically in both mice and human, we could now address the role of FBs by making use of pluripotent genetically engineered ESC lines.

In mice, we made use of a genetically engineered mESC line, containing the expression of the neomycin resistance cassette under the control of the CM-restricted $\alpha$-myosin heavy chain promoter ( $\alpha$ MHC-NeoR; Klug et al., 1996). mESCs were initially differentiated in spinner flasks for 11 days, and CMs were selected under Geneticin (G418) treatment $(400 \mu \mathrm{g} / \mathrm{ml})$ in order to obtain CM-enriched cell populations (mCMs; Figure 12). After 17 days of culture in spinner flasks, we were able to obtain highly purified mCMs $(93 \pm 1.7 \% \alpha$-actinin [ACTN2] positive; $n=6)$ as determined by flow cytometry (FC; Figure 12D). 
A

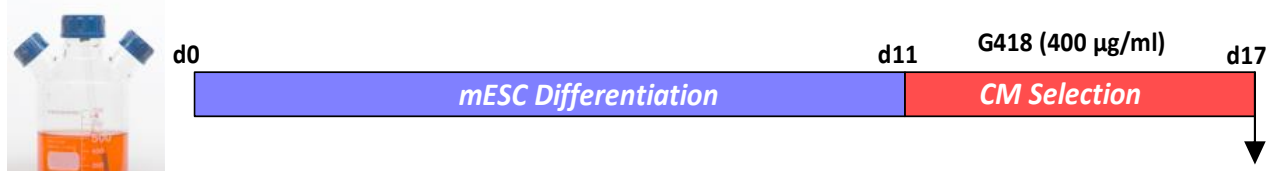

EB harvesting

mESC seeding

$\left(10 \times 10^{6}\right.$ cells/flask $)$

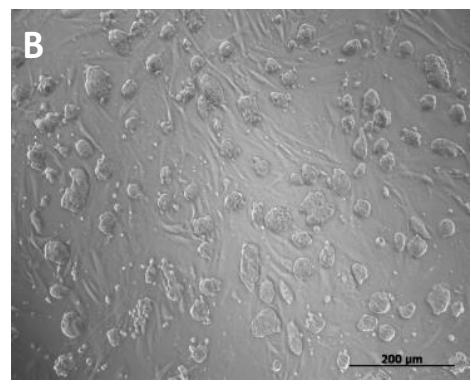

D

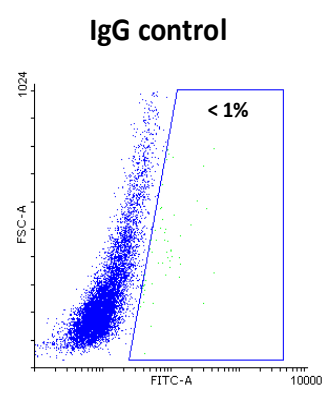

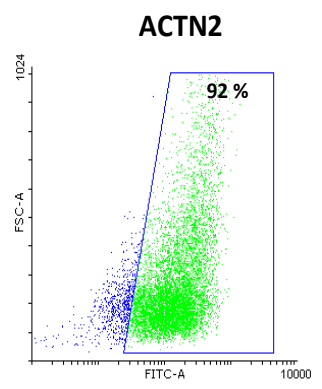

$\mathbf{E}$

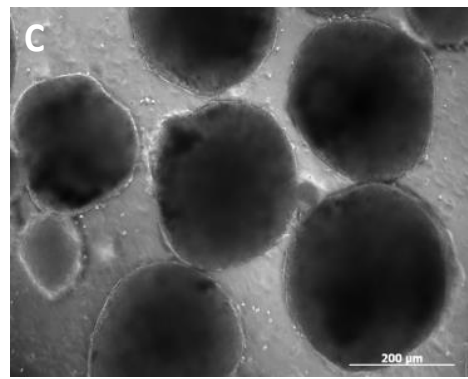

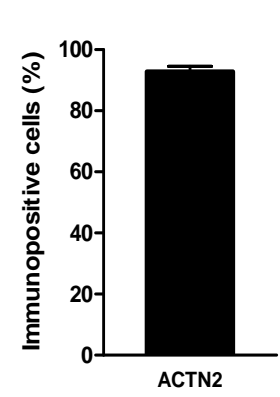

Figure 12. CM differentiation and purification of aMHC-NeoR mESCs. (A) Undifferentiated mESCs $\left(\alpha\right.$ MHC-NeoR) were seeded into spinner flasks $\left(10 \times 10^{6}\right.$ cells/flask $)$ and differentiated for 11 days in culture with constant stirring to generate embryoid bodies (EBs). CM selection at d11 was initiated with the addition of G418 (400 $\mu \mathrm{g} / \mathrm{ml})$ to eliminate non-myocytes. At d17, EBs were harvested and dissociated into single CMs. (B) Morphology of undifferentiated mESC colonies cultivated on irradiated MEF feeder layers, prior to differentiation. (C) Morphology of EBs on d17 of cardiac differentiation and selection, prior to harvesting. (D) Representative FC scatter plot for IgG (control) and ACTN2 populations from a CM differentiation run. (E) FC analysis demonstrated effective enrichment of CMs to $93 \pm 1.7 \% \alpha$-actinin positive (n=6).

Specifically in human, we differentiated genetically engineered hES2-RFP cells (Irion et al., 2007) into purified CMs (hES2-CM) using recently established and robust 2D cardiac differentiation (Hudson et al., 2012) and purification (Tohyama et al., 2013) protocols (Figure 13). After 23 days of culture, we were able to obtain highly purified hES2-CMs ( $80 \pm 1.4 \%$ ACTN2 positive; $n=4)$, as determined by FC (Figure 13B). 
A

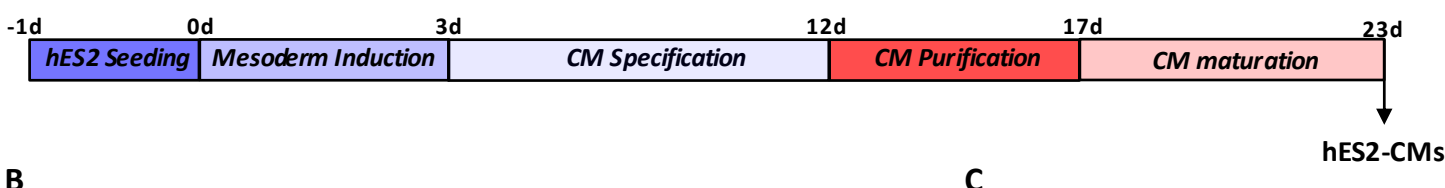

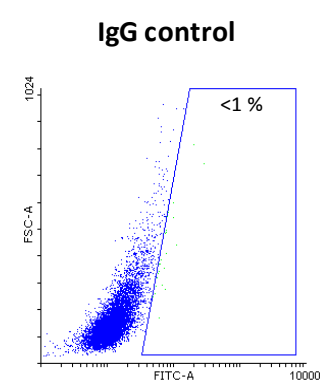

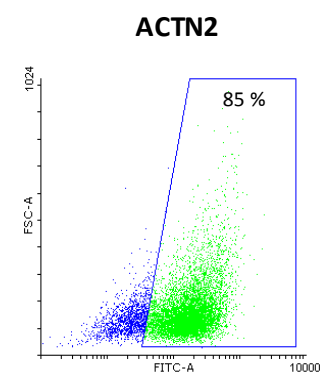

C

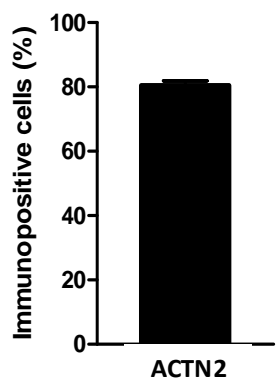

Figure 13. CM differentiation and purification of hES2-CMs. (A) Undifferentiated single cell adapted hES2 colonies were subjected to a cardiac differentiation and purification protocol to generate enriched hES-CMs. Briefly, undifferentiated hES2 cells were exposed to: Mesoderm induction (3 days), cardiac specification (8 days), metabolic purification (5 days, to eliminate non-myocytes) and cardiac maturation (6 days). Enriched hES2-CMs were subsequently harvested and dissociated into single cells. (B) Representative FC scatter plot for IgG (control) and ACTN2 populations from a CM differentiation run. (C) FC analysis demonstrated enrichment of hES2-CMs to $80 \pm 1.4 \%$ ACTN2 positive $(n=4)$.

\subsubsection{Cardiac fibroblasts are essential for EHM formation and maturation}

Purified mCMs were mixed with acetic acid solubilized rat tail collagen type I after $\mathrm{pH}$ neutralization with $\mathrm{NaOH}$ (mCM only) to investigate whether this environment alone was sufficient to drive maturation in mCMs. The predominant constituent of the myocardial ECM is collagen, and collagen type I accounts for approximately $80 \%$ of total myocardial collagen (Weber, 1989). The generation of EHMs (as outlined in Figure 10; Materials and Methods) supplemented with highly pure populations of mCMs were not able to compact into functional tissue and formed a 'spongy-like' phenotype (Figure 14A). Immunoflourescence imaging demonstrated the mCMs (green) within these EHMs stayed isolated with no tendency to form a functional syncytium, as indicated by the majority of immature round shaped CMs. EHMs were next generated with mixed populations of cFBs isolated from neonatal mouse hearts and purified mCMs (75\% mCMs; $25 \% \mathrm{cFBs})$ to investigate whether cFBs are 
important for tissue formation and maturation in EHM. EHMs supplemented with the addition of cFBs resulted in advanced condensation of EHMs (Figure 14B). mCMs within these EHMs formed tightly packed anisotropically organized muscle bundles and matured from an embryonic round shape into a typical postnatal rod-shaped phenotype with characteristic sarcomeric cross-striations. In addition, cFBsupplemented EHMs developed contractile forces analysed by concentration response measurements under increasing extracellular $\mathrm{Ca}^{2+}$ (Figure 14C). EHMs comprised of pure mCMs (only) failed to develop any contractile forces. These results support previous studies from our group (Figure S1, Appendix A1).

A

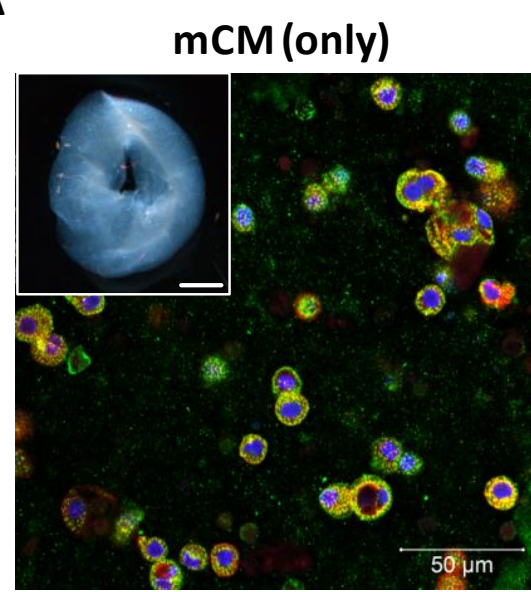

B

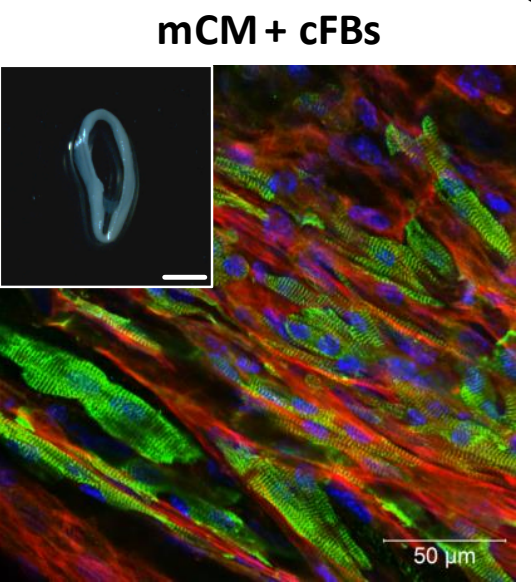

C

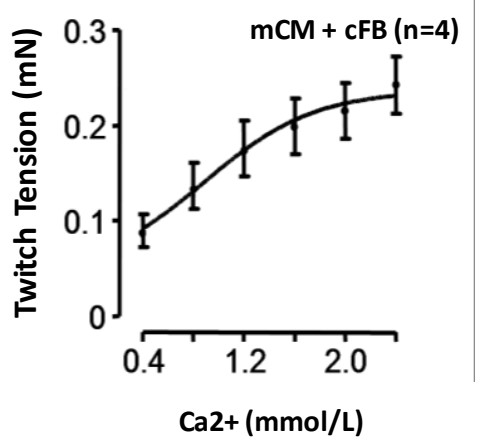

a-actinin/F-actin/Hoechst

Figure 14. Advanced mCM maturation in cFB-supplemented EHMs. (A) EHMs generated with enriched mCMs (only) after 10 days culture formed a non-condensed spongy-like tissue with rounded immature mCMs (green: $\alpha$-actinin). (B) Supplementation of cFBs ( $25 \%$ of total EHM composition) facilitated the formation of the typical compact EHM ring structure. mCMs developed into tightly packed and longitudinally orientated muscle bundles. Inset scale bars: $2 \mathrm{~mm}$. (C) Twitch tension from cFB-supplemented EHMs ( $\mathrm{n}=4$ EHMs), analysed by concentration response measurements under increasing concentrations of extracellular $\mathrm{Ca}^{2+}(\mathrm{mmol} / \mathrm{L})$. No contractile force was developed in EHMs generated from enriched mCMs only.

This data collectively suggests that cFBs are essential for tissue formation and CM maturation in our EHM model of heart muscle development. Similar results were observed when highly purified hES2-CMs were supplemented with human cardiac FBs or human foreskin FBs, in the human EHM model (Tiburcy et al., unpublished data). 


\subsection{ECM-integrin crosstalk in fibroblasts is responsible in mediating tissue formation in EHMs}

Interactions between cells and the ECM are mainly regulated via heterodimeric integrin receptors composed of $\alpha$ - and $\beta$-subunits. Integrins physically link the ECM to the cytoskeleton and are thereby able to translate extracellular signals into biochemical responses (Ross and Borg, 2001). In consequence, mechanisms of mechanotransduction are important not only for tissue homeostasis, but also to orchestrate the development of multicellular organs and tissues. Given the facts that purely-enriched mCMs alone failed to form compact, functional cardiac tissue in our EHM model, we wanted to know whether FB-specific mechanisms of mechanotransduction are responsible for the initial formation and maturation of EHMs in both mouse and human model systems.

\subsubsection{Collagen binding integrins are highly expressed in fibroblasts}

As collagen type I is the major component of the myocardial ECM and employed for the initial entrapment of cells in the EHM model, the expression of collagen binding integrin chains $\alpha 1, \alpha 2$ and $\beta 1$ (ITGA1, ITGA2 and ITGB1) was analysed in both mouse and human purified CM and FB fractions (Figure 15). As it is not clearly understood whether different types of FBs can improve tissue quality; FBs from both embryonic and cardiac origin were used in this study. Both $\alpha$ subunits are associated with ITGB1 to form functional receptor complexes $(\alpha 1 \beta 1$ and $\alpha 2 \beta 1)$ known to specifically bind to native collagen I (Langholz et al., 1995; Terracio et al., 1991; Kirchhofer et al., 1990).

Immunoblotting analysis revealed that collagen binding integrins; ITGA1, ITGA2 and ITGB1 were more abundantly expressed in both mouse neonatal cFBs and MEFs compared to purely-enriched mCMs (Figure 15B and C). Cross-contaminations of the analysed FBs and CM populations appeared negligible; based on the identification of FSP-1 exclusively in FBs populations, and calsequestrin (CASQ) and cardiac troponin $\mathrm{I}(\mathrm{CTNI})$ exclusively in purified CM populations. 
Likewise, immunoblotting analysis revealed the expression of collagen binding integrins; ITGA2 and ITGB1 were more abundantly expressed in human FB populations (hcFBs and hFFBs; Figure 15D and E). Procollagen 1 (ProCol1) was found to be exclusively expressed in human fibroblast populations and both CTNI and CASQ in hES2-CM populations. We did not however identify a specific antibody for the detection of ITGA1 in human samples (Figure S2, Appendix A1).

A

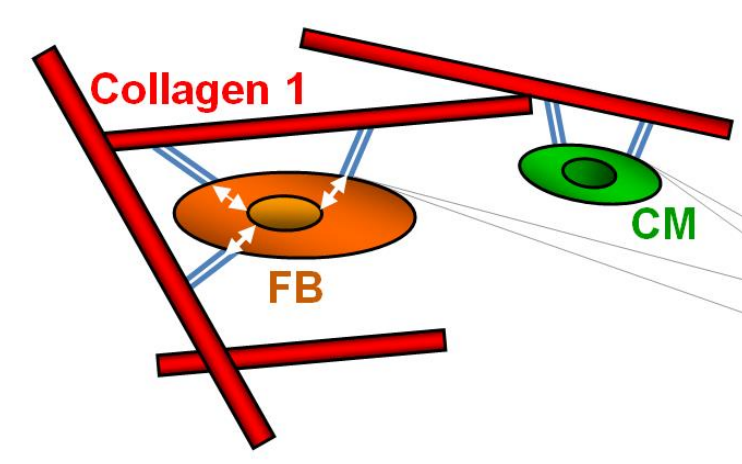

Integrin receptor (Itg)

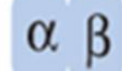

B

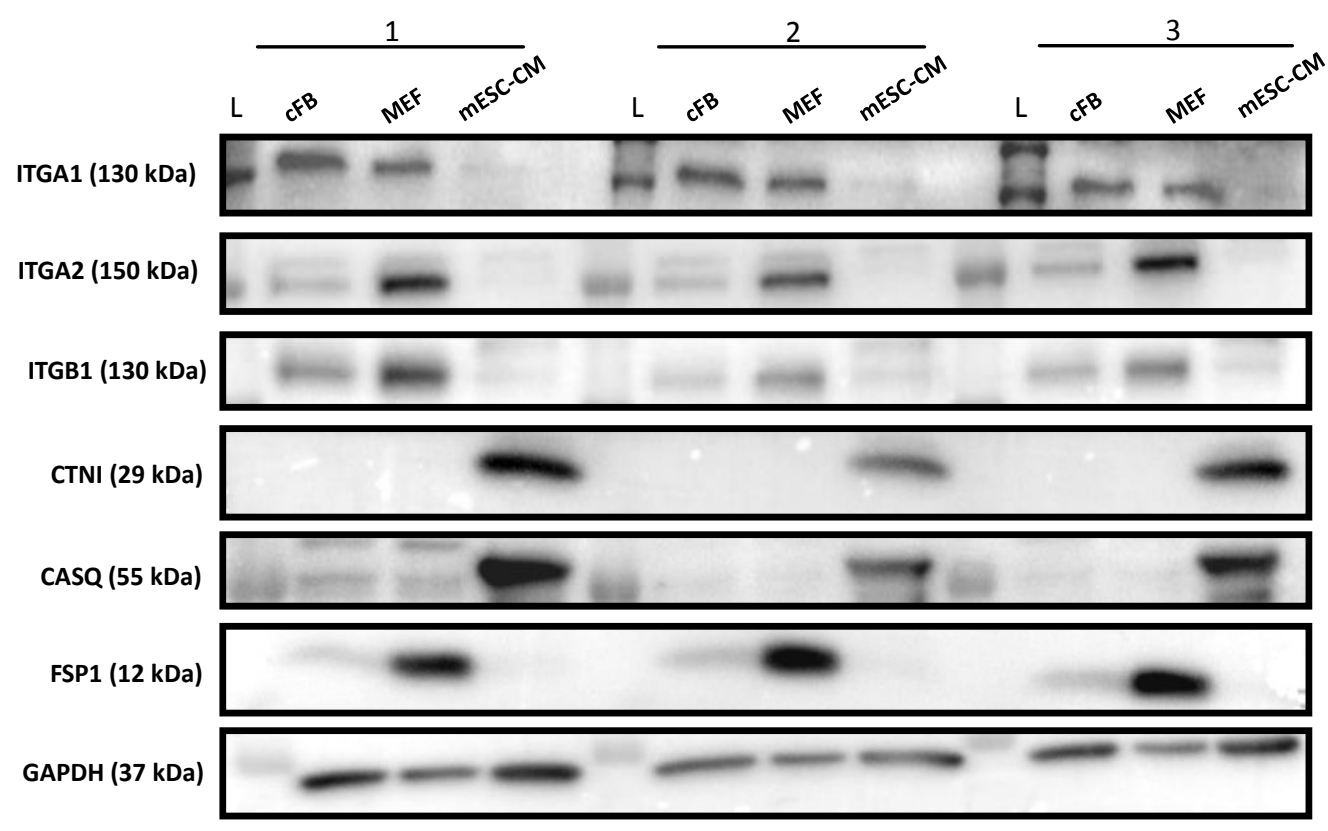

C
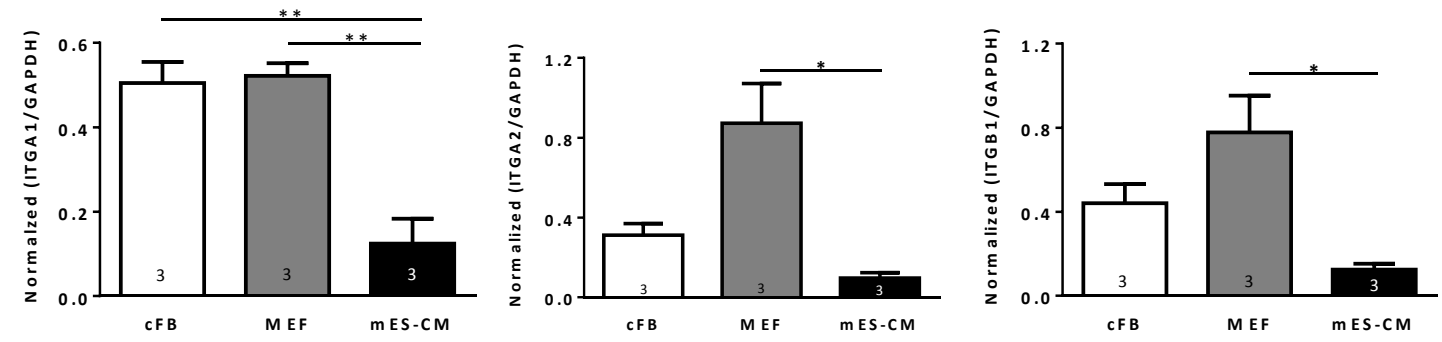


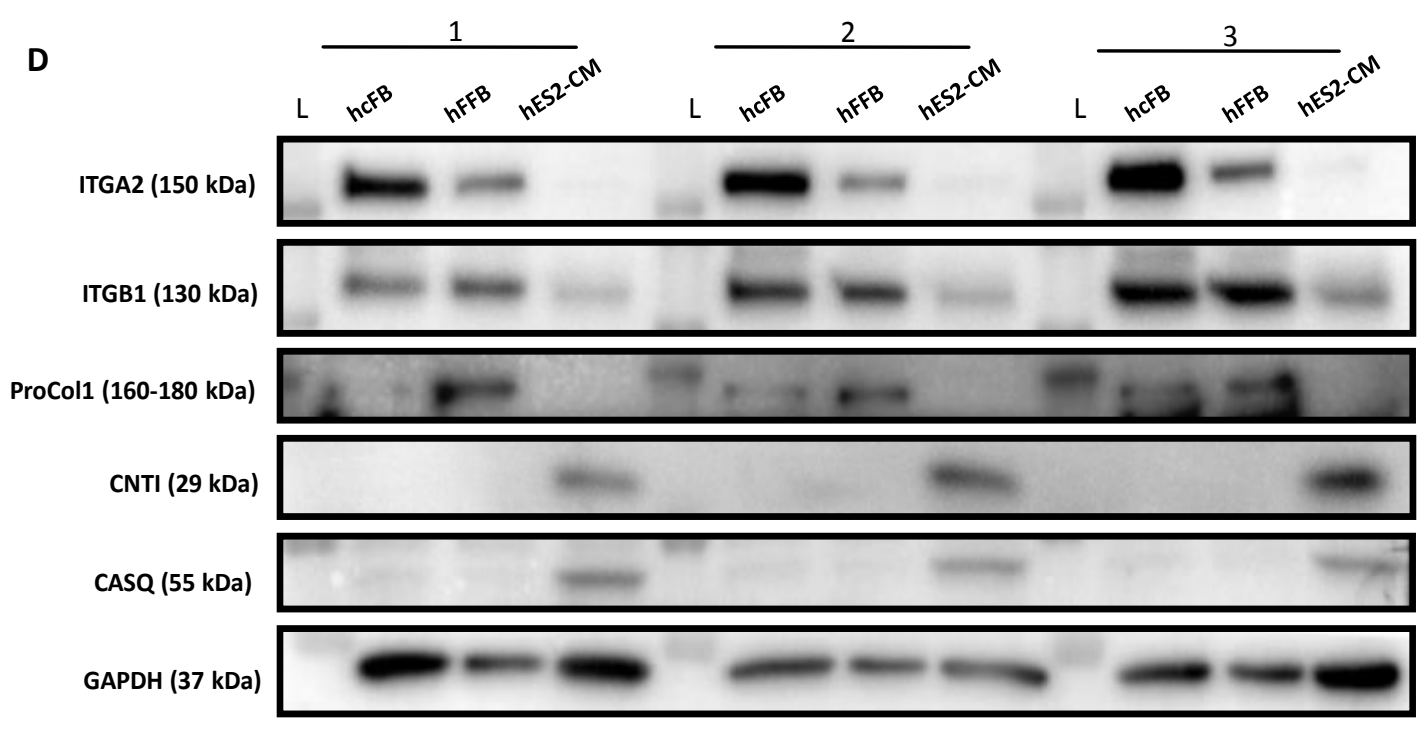

E
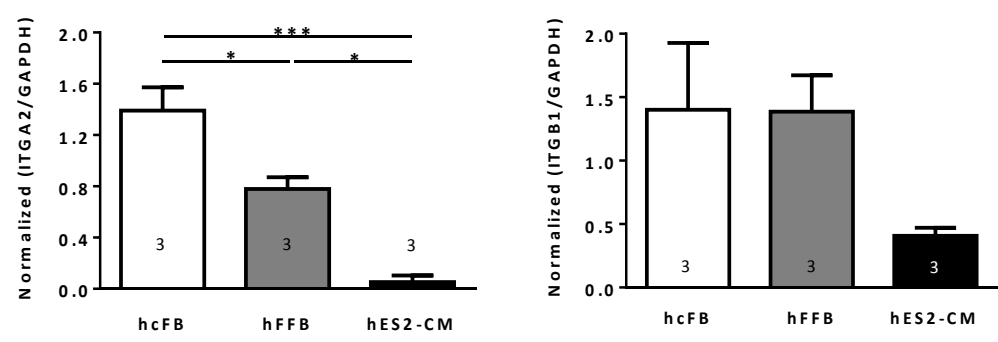

Figure 15. Collagen type I binding integrins ITGA1, ITGA2 and ITGB1 are highly expressed in mouse and human FBs. (A) Simplified schematic diagram illustrating the interaction of FBs and CMs with ECM Collagen type I in our 3D EHM model of heart muscle development. The interaction between heart cells (FBs and CMs) and collagen type I (red) are regulated via transmembrane heterodimeric integrin receptors composed of $\alpha$ and $\beta$ subunits (blue). (B) Western blot analysis of collagen type I binding integrins; ITGA1, ITGA2 and ITGB1 in mouse cell FB populations (mcFB and MEFs) and purely enriched mCM populations (n=3/group). Purity of investigated cell populations was demonstrated by cell type specific expression of CTNI, CASQ and FSP-1. 'L': Protein molecular weight ladder. (C) Densitometry analysis of ITGA1, ITGA2 and ITGB1 expression levels in mouse cell populations, normalized to GAPDH ( $\mathrm{n}=3$ samples/group; $* \mathrm{P}<0.05 ; 1$-way ANOVA with Bonferroni post-hoc test). (D) Collagen binding integrin expression in human FB populations (hcFB and hFFB) and purely enriched hES2-CMs (n=3/group). Purity of investigated cell populations was demonstrated by cell type specific expression of ProCol1, CTNI and CASQ. (E) Densitometry analysis of ITGA2 and ITGB1 expression levels in human cell populations, normalized to GAPDH ( $\mathrm{n}=3$ samples/group; $* \mathrm{P}<0.05 ; 1$-way ANOVA with Bonferroni post-hoc test). 
Furthermore, the abundance of Itgal, Itga2 and Itgbl transcripts in mouse cells (cFB, MEF and mCM populations) were analysed via qPCR. qPCR analysis comfirmed Itgal and Itga2 transcripts to be of low abundance in mCMs $(* \mathrm{P}<0.05 ; \mathrm{n}=\geq 3$ samples/group) (Figure 16A and 16B) compared to MEFs, however this was not significantly different compared to cFBs. Itg $\beta 1$ transcripts were significantly more abundant ( ${ }^{*} \mathrm{P}<0.05 ; \mathrm{n}=\geq 3$ samples/group) in embryonic and cardiac FB populations (MEF and cFB) compared to mCMs (Figure 16C).

A

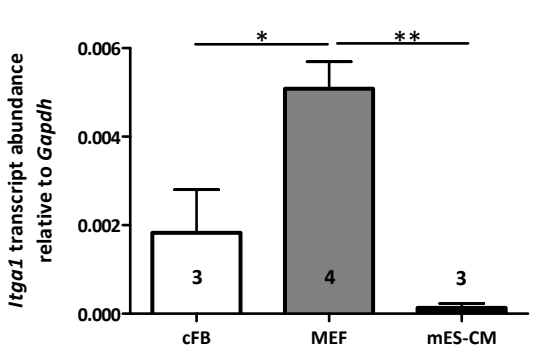

B

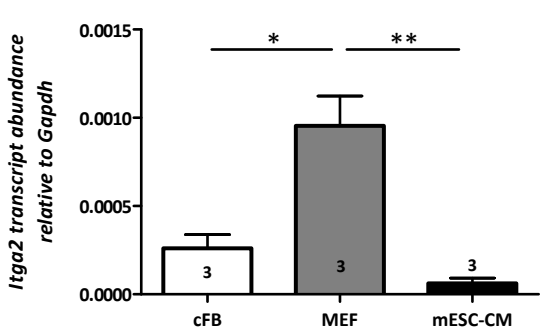

C

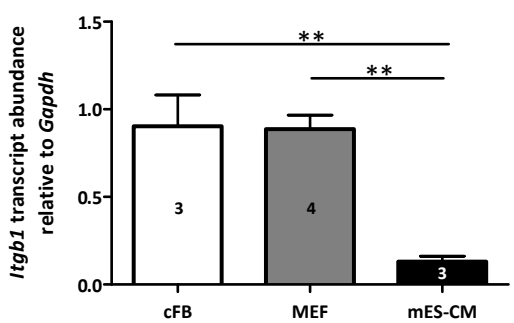

Figure 16. Higher abundance of collagen-binding Itgal, Itga2 and Itgb1 subunit transcripts in FB populations. qPCR analysis of (A) Itgal, (B) Itga2 and (C) Itgb1 transcripts in FB populations (cFBs and MEFs) and mESC-enriched CMs, relative to GAPDH (samples/group as indicated in the bars; $* \mathrm{P}<0.05$; 1-way ANOVA with Bonferroni post-hoc test).

RNA-seq transcriptome analysis was performed on individual human FB populations ( $\mathrm{hFFB}$ and $\mathrm{hcFB}$ ) and $\mathrm{hES} 2$-enriched $\mathrm{CM}$ populations ( $\mathrm{n}=3$ /group) to assess the abundance of individual integrin subunits associated with collagen, laminin, and RGD-receptors. This data confirmed that all transcripts encoding for collagen-binding integrins, including; ITGA1, ITGA2 and ITGB1, were highly abundant in FB populations over $\mathrm{CM}$ populations (Figure 17A). In addition, cell type specific transcript assessments demonstrate that both FB-associated markers (Vimentin [VIM], FSP-1 [S100A4], DDR2, CD90 [THY1], Periostin [POSTN] and Transcription factor 21 [TCF21]) and CM-specific markers (TNNT2, ACTN2, MYH6 and MYH7) were exclusively expressed in human FB populations and enriched-CM populations, respectively (Figure 17B). The purity of human $\mathrm{CMs}$ was confirmed by $\mathrm{FC}$ for ACTN2 (93 $\pm 3.8 \% ; n=3)$ (Figure 17C). 
A

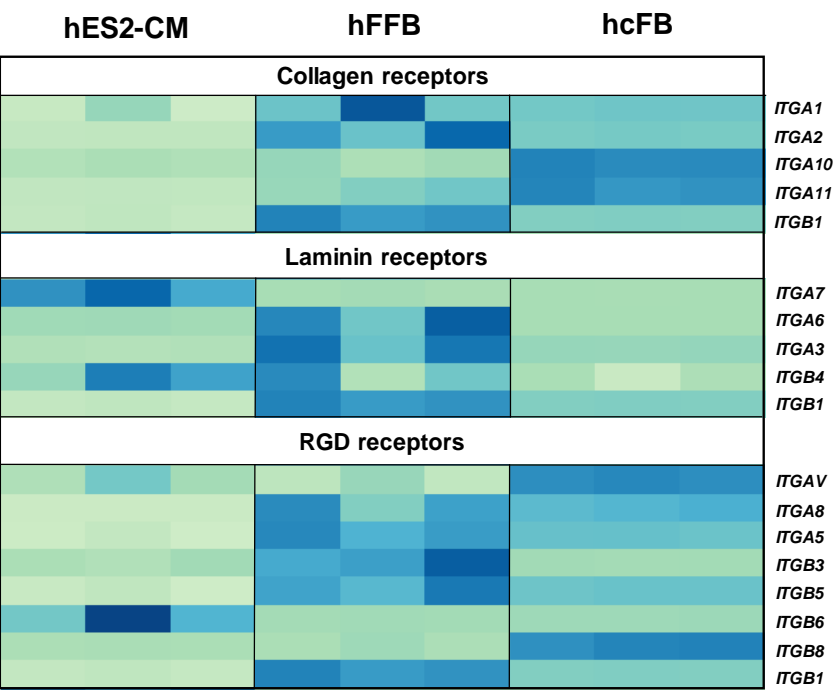

B

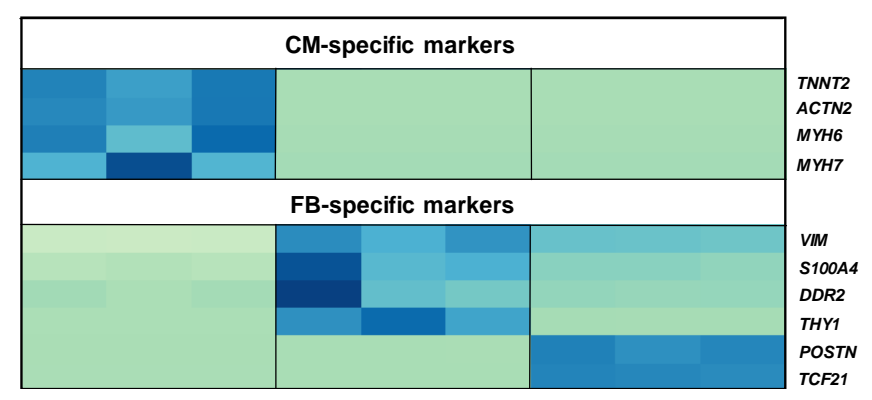

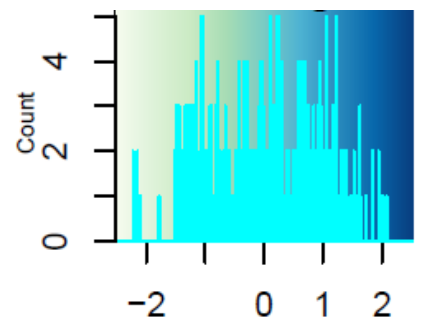

C

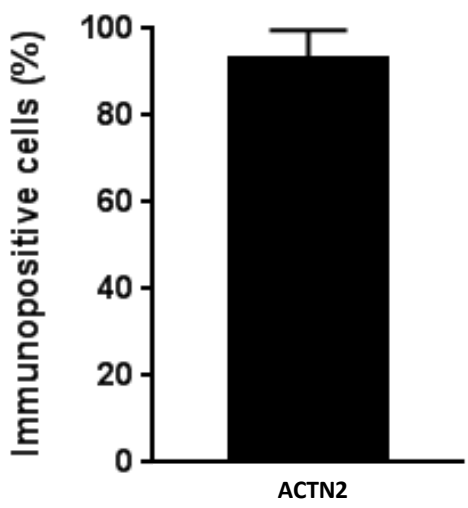

Figure 17. Integrin subunit transcript abundance in hES2-enriched CMs and FB populations. (A) Heat map illustrating $\log 2$ fold change expression values of integrin subunit transcripts identified by RNA-seq analysis. Collagen receptor integrin subunits were of higher abundance in human fibroblast populations (hFFB and hcFB) compared to purely enriched hES2-CMs (n=3/group). Darker blue shadings on the heat map represent higher transcript abundance. (B) FB- and CM- associated transcript markers were highly abundant in FB populations (VIM, S100A4, DDR2, THY1, POSTN and TCF21) and CM populations (TTNT2, ACTN2, MYH6 and MYH7), respectively. (C) FC analysis confirmed hES2-CM enrichment ( $\mathrm{n}=3$ samples).

RNA-seq analysis furthermore revealed that human FB populations do not express laminin-specific Itg $\alpha 7$ in contrast to human CMs. This could be explained due to the fact that FBs have no laminin-containing basement membrane (Ross and Borg, 2001). RNA-seq analysis furthermore revealed that all transcripts encoding for RGD receptors were of low abundance in $\mathrm{CM}$ populations compared to FB populations, with exception to ITGB6. 


\subsubsection{Blocking integrin-collagen type I interactions abolishes initial EHM} formation

Next, we investigated the role of ITGA1, ITGA2 and ITGB1 mediated signalling in the initial phases of EHM formation in mouse and human cells. Mixed populations of single cells derived from purely enriched CMs and FBs (75: 25 ratio respectively) were treated for 30 mins with blocking antibodies targeting individual ITGA1 (CD49a), ITGA2 (CD49b) and ITGB1 (CD29) chains respectively, prior to the generation of EHMs. For controls, cells were treated with IgG antibody. For negative controls, EHMs were generated containing only purely enriched mCMs, which fail to form compact tissue. To enable parallel screening for assessing the effects of each integrin blocking antibody on EHM formation, both EHM cell suspension/reconstitution mixture volumes were scaled down and cast onto individual wells of 96-well low attachment plates $\left(1.25 \times 10^{5}\right.$ cells/well) for initial tissue formation (Figure 18A). Low attachment plates were used to prevent cell suspensions/tissues from adhering and interfering with the bottom and sides of the plates. After $1 \mathrm{hr}$ of initial EHM condensation, medium (containing the respective blocking antibody) was added to each casting EHM well and tissues were cultured for a further $20 \mathrm{hrs}$. The level of tissue retraction after $21 \mathrm{hrs}$ ('blocking') from initial EHM casting was then evaluated in each well. The antibody was subsequently washed out from EHMs and tissue contraction was assessed after a further 72 hrs ('recovery'). The surface area for each EHM relative to the total surface area in each well (\%) was analysed during both 'blocking' and 'recovery' phases. 
A

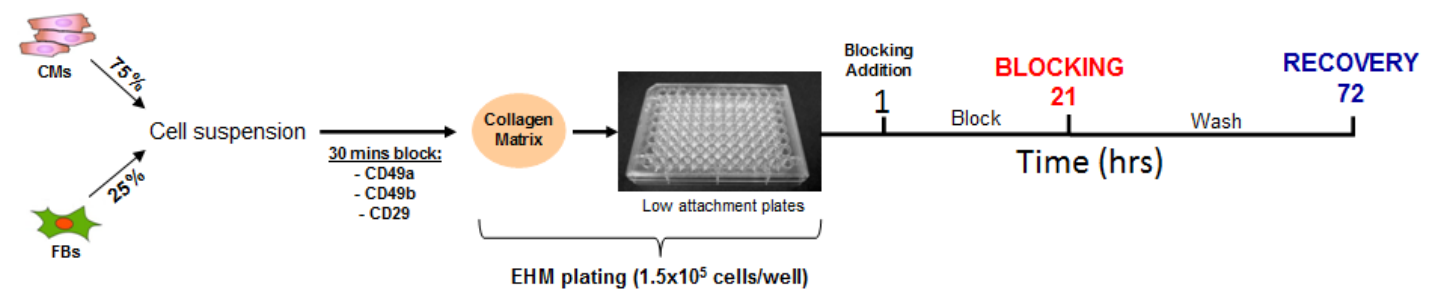

B
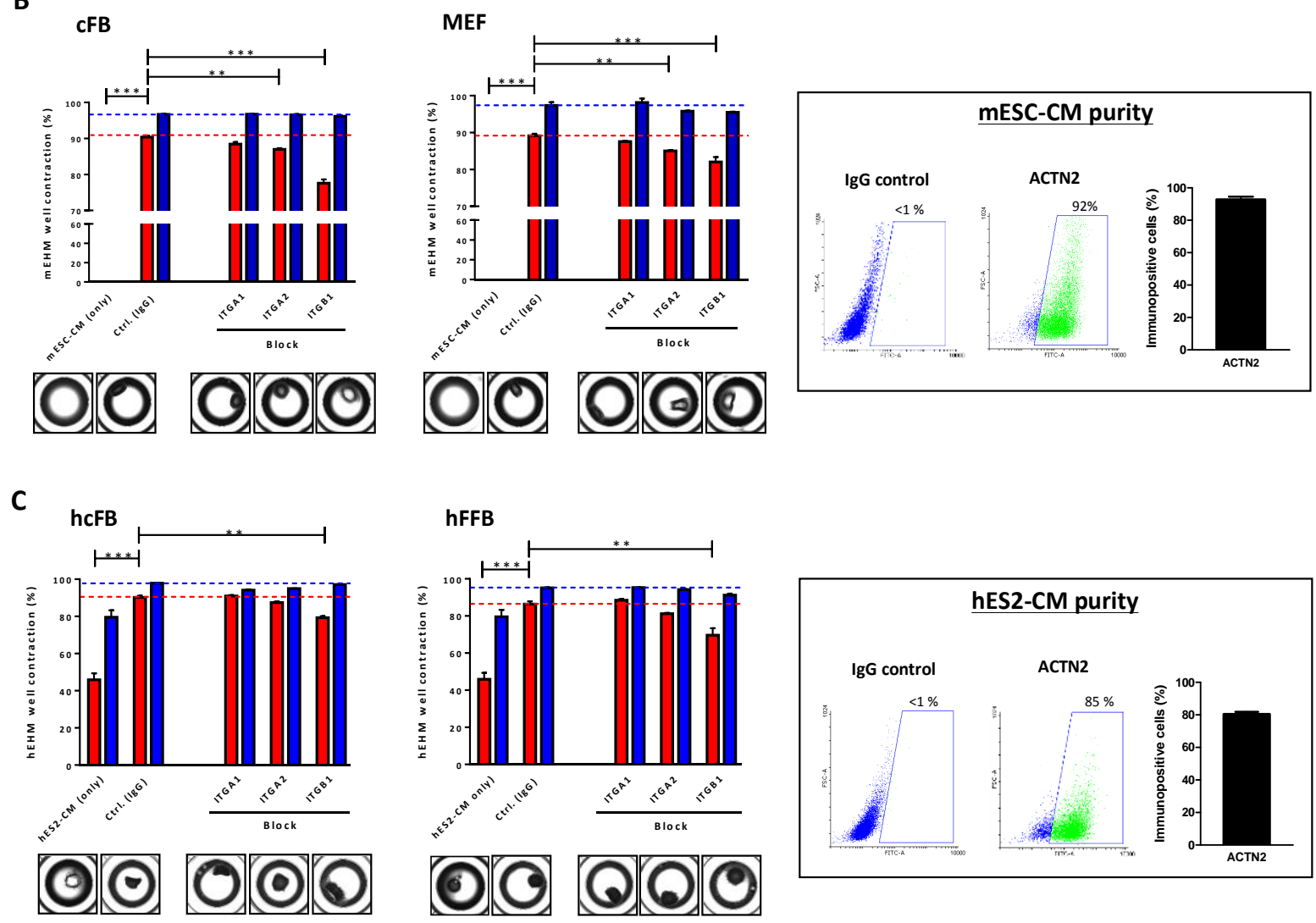

Blocking (21hrs)

Recovery (72hrs)

Figure 18. Integrin-mediated signalling in FBs facilitated EHM formation. (A) Schematic representation of the experimental design. Cell suspensions (FBs and CMs) and EHMs were incubated with individual blocking

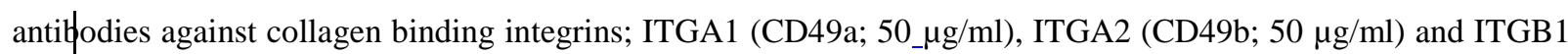
(CD29; $40 \mu \mathrm{g} / \mathrm{ml})$. The effects of 'blocking' and 'recovery' on EHM formation were assessed via optical feature tracking (Cellavista). (B) The blocking of ITGA1, ITGA2 and ITGB1 inhibited mouse EHM formation after 21 hrs (red), as indicated by the \% of EHM area relative to the surface area of each well. These effects were strongly pronounced with Itg $\beta 1$ blocking ( ${ }^{*} \mathrm{P}<0.05$; 1 -way ANOVA with Bonferroni post-hoc test). EHMs comprised of mCMs (only) failed to form compact tissue. Similar effects were observed with EHMs comprised of MEFs and mCMs. The images below each graph display a representative EHM per well for each different condition, after 21 hrs blocking. Representative FC scatter plot for IgG (control) and ACTN2 populations from a mESC enrichedCMs used for experiments. FC analysis demonstrated that populations of purely enriched CMs were on average $93 \pm 1.7 \%$ ACTN2 positive $(n=6)$. (C) Using a similar experimental design as in (B), we confirmed the mouse data|in a human model. Note that the CM enrichment was less optimal with $80 \pm 1.4_{-} \%$ ACTN2 positive CMs in this experimental series $(n=4)$, explaining the condensation in the hES2-CM (only) group. 
For mouse cells, in neonatal cFB-supplemented EHMs, blocking of the ITGB1collagen type I interaction significantly abolished the initial EHM formation (4 EHMs/group; $* \mathrm{P}<0.05)$, indicating that ITGB1 is involved in collagen type I matrix condensation and necessary for EHM formation. In addition, blocking of the ITGA2collagen type I interaction also significantly abolished initial EHM formation (4 EHMs/group; $* \mathrm{P}<0.05)$, but to a lesser extent than with ITGB1 blockade. Lastly, blocking of ITGA1-collagen type I interactions partially inhibited EHM formation however this was shown to be not significant compared to IgG-treated controls. The effects of the blocking antibodies on EHM condensation were reversible with antibody washout after $72 \mathrm{hrs}$ (recovery), indicating that inhibition of tissue retraction was not due to cell death. EHMs comprised of purely enriched CM only (as determined via FC; Figure 18B) failed to contract and form compact tissue throughout the entire duration of tissue culture. Since the ITGB1 chain subunit is present in all collagen binding (as well as Laminin and RGD binding) integrin heterodimeric receptor complexes, these dramatic effects we observed with ITGB1 blockade on tissue formation most likely influencing other important integrin heterodimeric receptors. Likewise, similar observations were made with MEF-supplemented EHM, supporting the notion that FB-specific mechanisms of mechanotransduction from different FB cell sources are responsible in mediating the initial tissue formation process in EHMs.

Next, integrin blocking experiments were subsequently performed using hcFB- and hFFB- supplemented EHMs (Figure 18C). Similarly, blocking of the ITGB1-collagen type I interaction significantly inhibited initial tissue formation (4 EHMs/group; $* \mathrm{P}<0.05)$ in both cFB and hFFB-supplemented EHMs. Blocking of ITGA2-collagen type I interactions partially inhibited EHM formation, however this was shown to not be significant compared to IgG-treated controls. Finally, blocking of the ITGA1collagen type I interaction did not show any inhibitory effects on EHM contraction after 21 hrs blocking treatment, compared to IgG-treated controls. Interestingly, EHMs comprised of enriched hES-CMs were able to partially contract after $21 \mathrm{hrs}$ blocking, and further after $72 \mathrm{hrs}$. This was most likely due to fact that these CM populations were not entirely enriched in ACTN2 immunopositive CMs (80 $\pm 1.4 \%$; $\mathrm{n}=4$ ). Taken together, these data collectively indicate that ECM-integrin mechanotransduction mediated via collagen, laminin and RGD-binding integrin 
receptors in FBs are essential for matrix condensation and tissue maturation in our EHM model of heart muscle development.

\subsection{Cardiac fibroblasts construct an ECM environment in EHMs}

Multicellular tissues including the heart are composed of a complex ECM architecture which is built by FBs. Since we have shown that FBs are essential for heart muscle formation and maturation in our EHM model, the following studies were performed to further investigate the influence of the FB-producing ECM environment in promoting the maturation of CMs in the EHM. The ECM composition was investigated in EHMs by transcriptomics and proteomics.

Explorative transcriptome profiling was performed at different points of EHM maturation and a massive up regulation of ECM-related transcripts was observed (Figure 19A) (Christalla et al., unpublished data). The collagen type I chains Collal and Colla2 were the most abundant transcripts. Besides collagen type I, other transcripts which are mainly involved in collagen I fibrillogenesis and maturation were also highly abundant, such as decorin (Dcn) and biglycan (Bgn) (Campbell et al., 2008), indicating that collagen type I modification is also important for EHM maturation. 

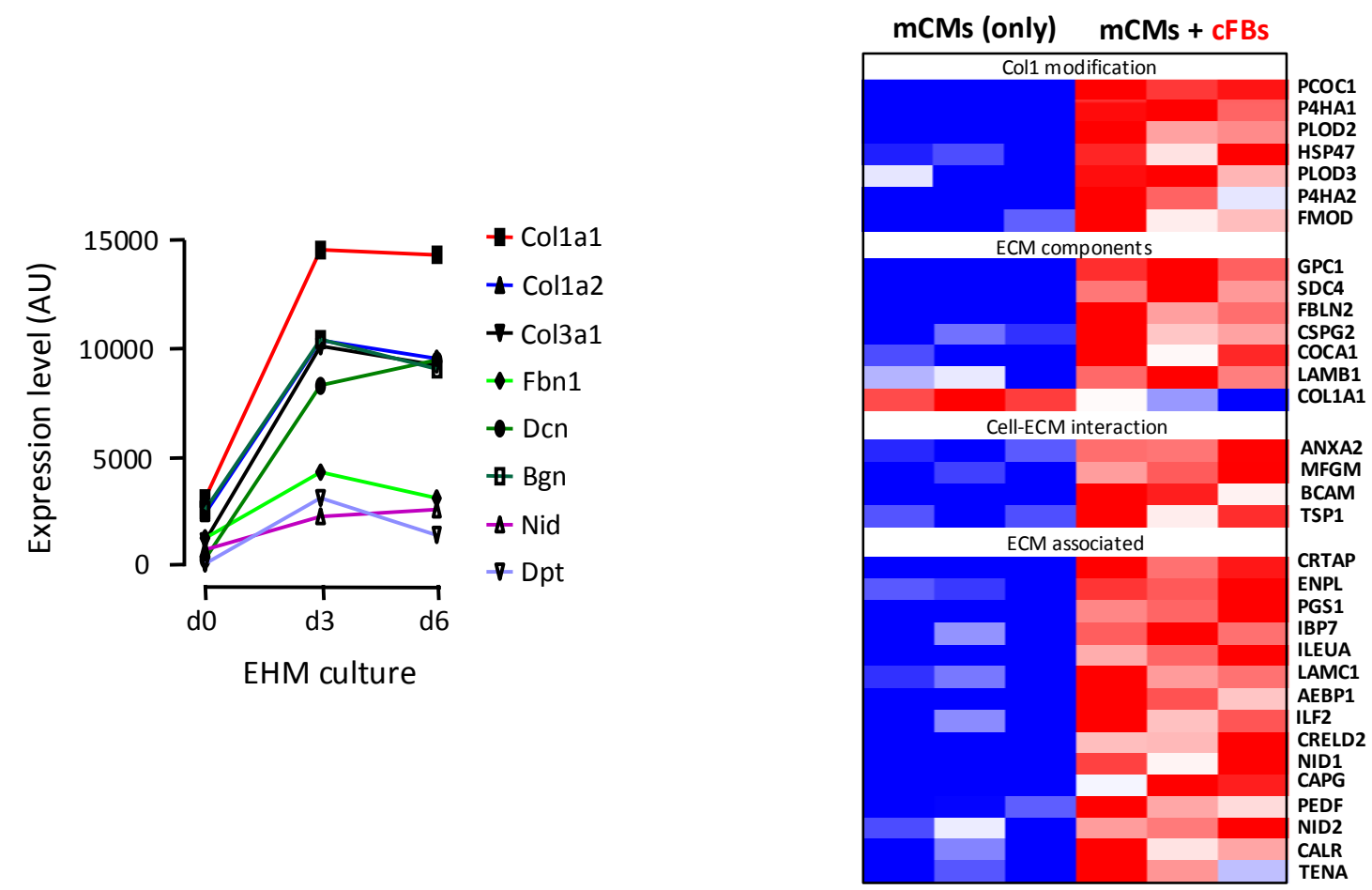

Figure 19. cFBs construct a heterogeneous ECM architecture in mouse EHMs. (A) Microarray analysis of the most abundant ECM-related transcripts during EHM maturation ( $n=1 /$ group). (B) Heat map showing differentially expressed ECM-related proteins identified by proteomics in d6 EHMs composed of mCMs alone vs. FB-supplemented EHMs (n=3/group). Heat map outlining differentially expressed proteins clustered into Collagen type I modifying proteins, ECM components, Cell-ECM interacting and ECM associated proteins. Red represents high protein abundance, blue represents low protein abundance. (Data from Christalla et al., unpublished data).

Next, the cFB-derived ECM components in EHMs were then analysed by proteomic profiling (Figure 19B). De novo synthesized ECM components and cellular proteins were fractioned and enriched from the original input collagen (used to initially generate EHMs) by salt $(\mathrm{NaCl})$, followed by subsequent SDS treatment (Didangelos et al., 2009). In total, 663 proteins were identified by liquid chromatography tandem mass spectrometry (LC-MS/MS). Interestingly, 33 differentially expressed ECMrelated proteins were only expressed in the FB-supplemented EHMs but not detectable in EHMs composed of purified mCMs only $(n=3 ; p<0.05)$. The detected COL1A1 expression by proteomics in EHMs containing only mCMs reflects the lack of ECM (collagen) remodelling in this condition. 
Furthermore, proteins which are involved in collagen type I modification and organisation, such as procollagen C-endopeptidase enhancer 1 (PCOC1), procollagenlysine 2-oxoglutarate 5-dioxygenase 2 (PLOD2) and PLOD3, Prolyl 4-Hydroxylase alpha polypeptide 1 (P4HA1) and P4HA2 were mainly expressed in FB-supplemented EHMs, which suggest these FB populations within the tissue reorganise the collagen matrix and are able to build a structural ECM environment. Taken together, these data indicate that cFBs are able to synthesize collagen modifying proteins, and are furthermore able to construct a heterogeneous ECM environment in our EHM mouse model of heart development.

Since mouse cFB-supplemented EHMs show an increase abundance of many ECMrelated proteins compared to EHMs comprised of only mCMs, we also wanted to observe for any differences in the human system, when comparing individual human $\mathrm{CM}$ and FB (hcFB and hFFB sources) populations. RNA-seq transcriptome analysis performed on purely enriched hES2-derived CMs, hcFBs and hFFB populations ( $n=3$ /group) demonstrate that (1) collagen modifying, (2) ECM component, (3) CellECM interacting, and (4) ECM associating transcripts were predominantly of higher abundance in human FBs popultations than in hES2-CMs (Figure 20), further supporting the notion that FBs from both mouse and human are able to construct an ECM cardiac microenvironment. Interestingly however, we identified that several laminin subtype transcripts: LAMA1, LAMA2 and LAMC3 were exclusively highly abundant in human CMs. LAMA4 appeared to not be expressed in both human CM and FB populations. 
hFFB hcFB

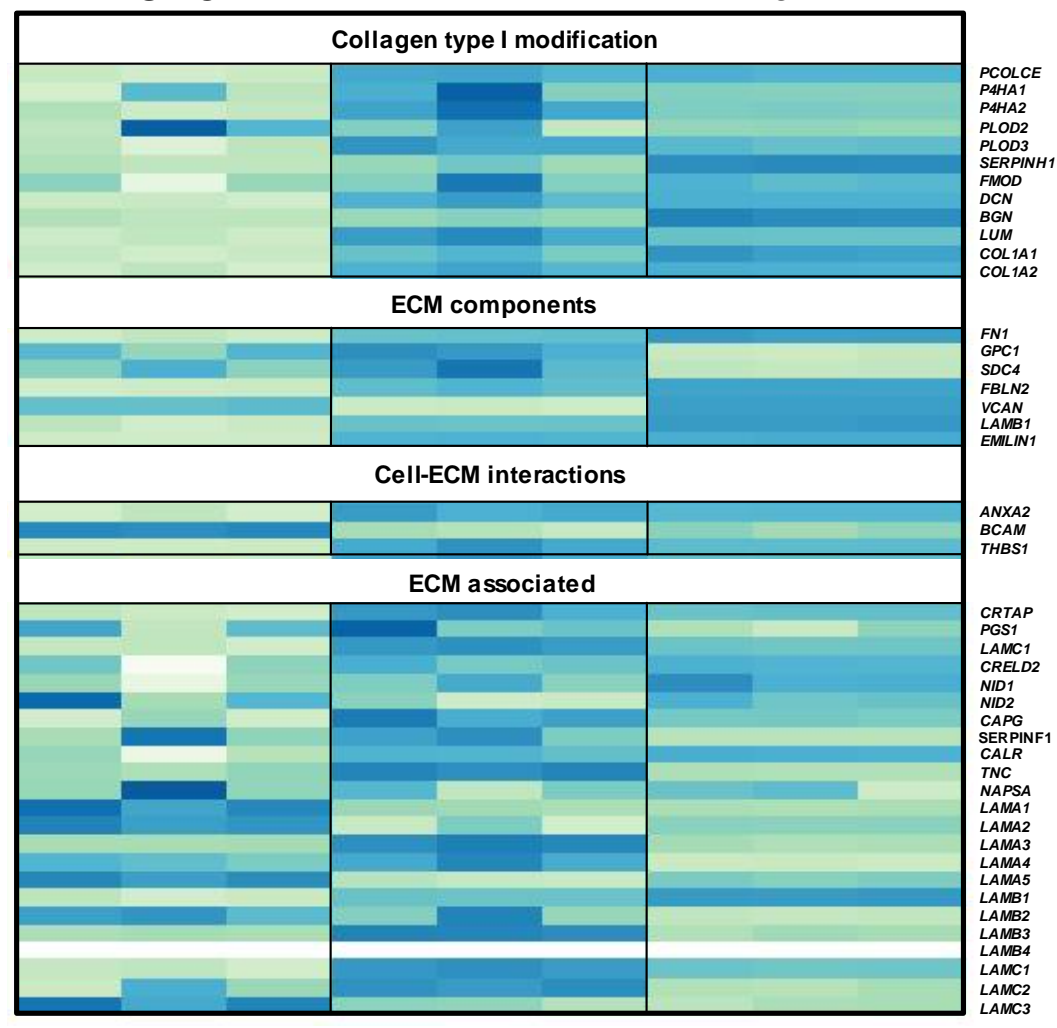

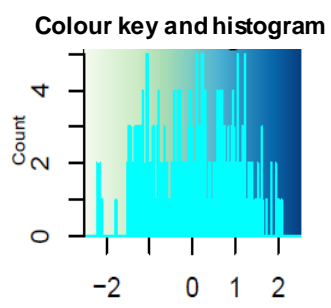

Figure 20. Collagen modifying and ECM-related transcripts are highly expressed in human FBs. Heat map illustrating $\log 2$ fold change expression values of ECM-related transcripts identified by RNA-seq analysis. Differentially expressed transcripts are clustered into; Collagen type 1 modifying proteins, ECM components, Cell-ECM interacting and ECM associated proteins. ECM-related transcripts were generally of higher transcript abundance in FB cell populations (hcFB and hFFBs; as outlined) compared to purely enriched hES2-CMs ( $\mathrm{n}=3$ /group). Darker blue shadings on the heat map represent higher transcript abundance.

\subsection{Influence of collagen processing HSP47 on cardiomyocyte maturation and homeostasis.}

It has previously been shown that HSP47 is a collagen specific ER molecular chaperone essential for the maturation of collagen type I and IV (Ishida et al., 2006). HSP47 KO mice die early during development showing abnormal orientated epithelial tissues and ruptured blood vessels (Nagai et al., 2001). Although the embryos from HSP47 KO mice show developmental retardation and exhibit cardiac hypertrophy, it is not clearly understood how HSP47 can influence the myocardium. Due to the fact that collagen type I is the major component of the myocardial ECM and that its 
organization seems to be a crucial factor for EHM maturation in our model of heart muscle development, we next scrutinized our transcription and protein data for the presence of HSP47 cell type specific expression, in CMs and FBs.

\subsubsection{Collagen processing HSP47 is highly expressed in fibroblast populations}

Since collagen processing HSP47 has been shown to be expressed in the ER of collagen-secreting cells (Nagata and Hosokawa, 1996; Taguchi and Razzaque, 2007) we wanted to evaluate its expression in both FB and purified CM populations in mouse and human cells. Immunoblotting analysis revealed that HSP47 (47 kDa) expression was higher in mouse FB populations (cFBs and MEFs) than in CM (Figure 21A), however this was not statistically significant. Furthermore, immunohistochemistry analysis of differentiated CM populations containing very small contaminating populations of non-myocytes (which do not express CM-specific cardiac troponin I type 3 [TNNI3] - green) demonstrate that non-myocytes exhibit a strong pattern expression of HSP47 (red), localized in the cellular cytoplasm surrounding the nucleus (Figure 21B). TNNI3-expressing CMs show at a weaker expression of HSP47. 
A

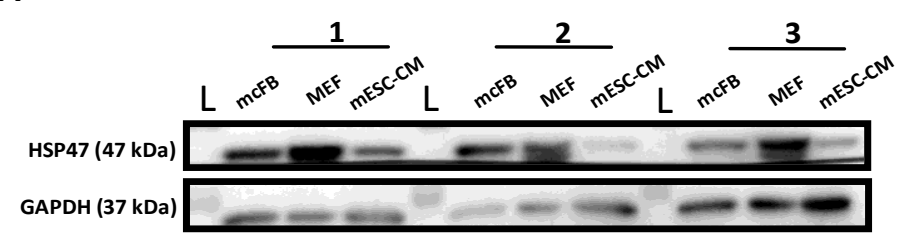

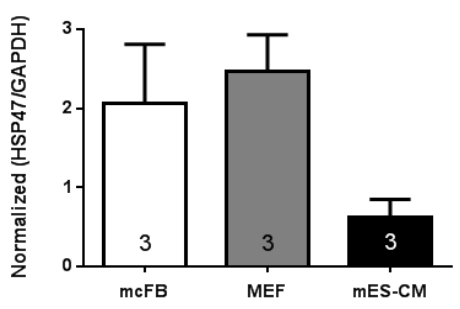

B
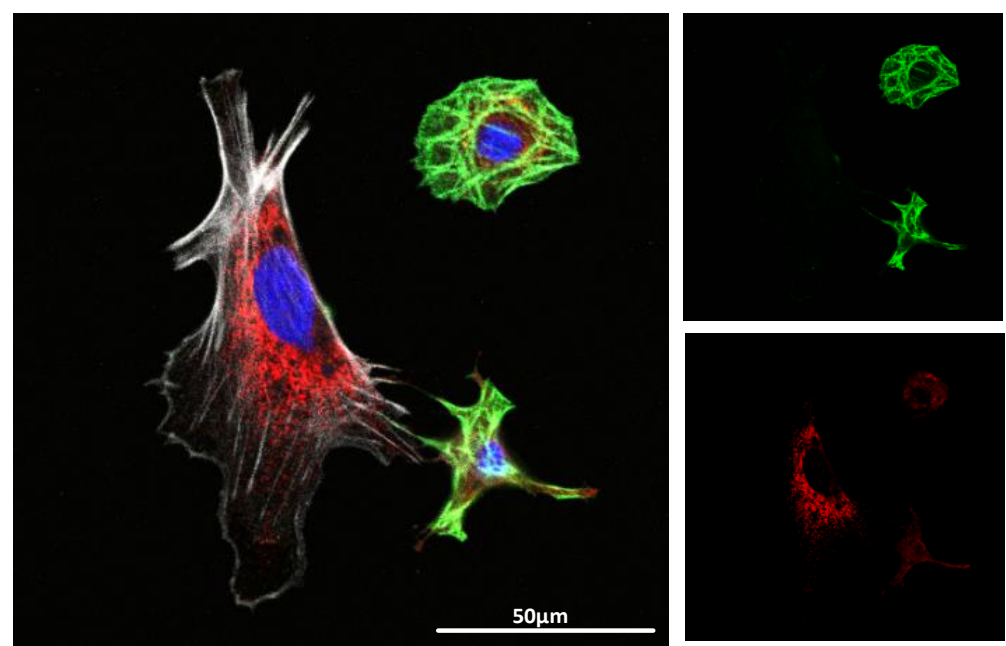

TNNI3, HSP47, F-actin, Hoechst

Figure 21. Collagen processing HSP47 is highly expressed in mouse FBs compared to mCMs. (A) Western blot analyses reveals greater expression of HSP47 in mouse FB populations (mcFBs and MEFs) compared to purely enriched mCMs ( $\mathrm{n}=3$ /group). 'L': Protein molecular weight ladder. (B) Immunostaining analysis of mCMs expressing CM-specific TNNI3 (green) revealed strong HSP47 expression patterns (red) localized in the cytoplasm of small contaminating non-myocyte fractions (not expressing TNNI3) from enriched mCM populations. Weak HSP47 expression was observed in TNNI3-expressing mCM, in line with the western blot data.

Next, we wanted to evaluate the expression of HSP47 in the human system. Immunoblotting analyses in human FB populations (hcFBs and hFFBs) also show greater expression of HSP47 than in hES2-CMs (Figure 22A). Likewise, immunohistochemistry analysis of differentiated hES2-CM populations (Figure 22B) also containing small contaminating populations of non-myocytes (which do not express TNNI3) demonstrated again that these cells exhibit a strong pattern expression of HSP47 localized in the cellular cytoplasm surrounding the nucleus. TNNI3-expressing hES2-CMs showed lower expression levels of HSP47. Since these differentiated hES2 populations were not as much enriched $(80.4 \pm 1.4 \%$ ACTN2 positive; $n=4$ ), we suspected that the contaminating presence of non-myocyte 
populations resulted in a stronger than anticipated HSP47 signal in the CM group. The RNA-seq analysis from even purer CMs populations $(93 \pm 3.8 \%$ ACTN2 positive; $\mathrm{n}=3$; Figure 17C) demonstrate significantly low SERPINH1 (HSP47) expression as compared to cFBs $(* \mathrm{P}<0.05 ; \mathrm{n}=3$ ) (Figure $22 \mathrm{C})$. On the contrary, hFFBs at protein level exhibited higher HSP47 expression compared to hcFBs. Interestingly, although purified CMs show negligible abundance of collagen type I transcripts (COLIAI and COL1A2) (Figure 22C), these cells however express HSP47, but at low protein and transcript level.

A
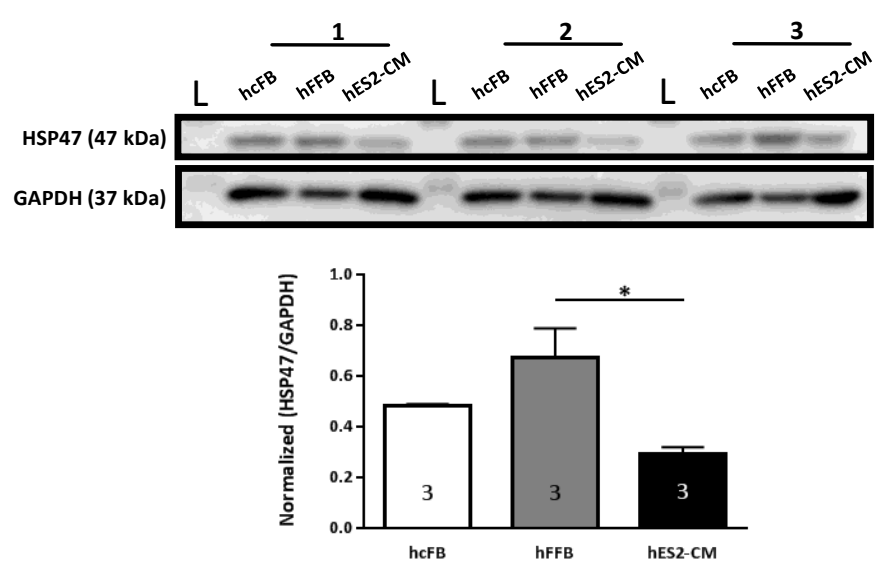

B

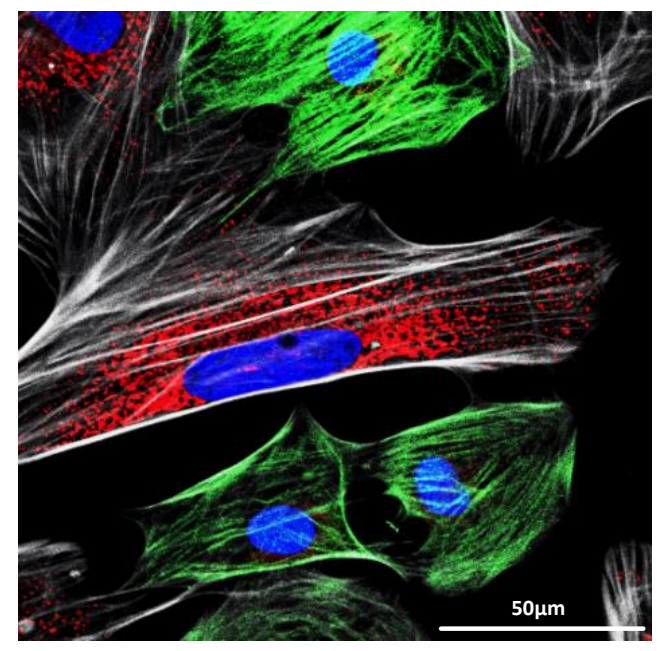

TNNI3, HSP47, F-actin, Hoechst

C
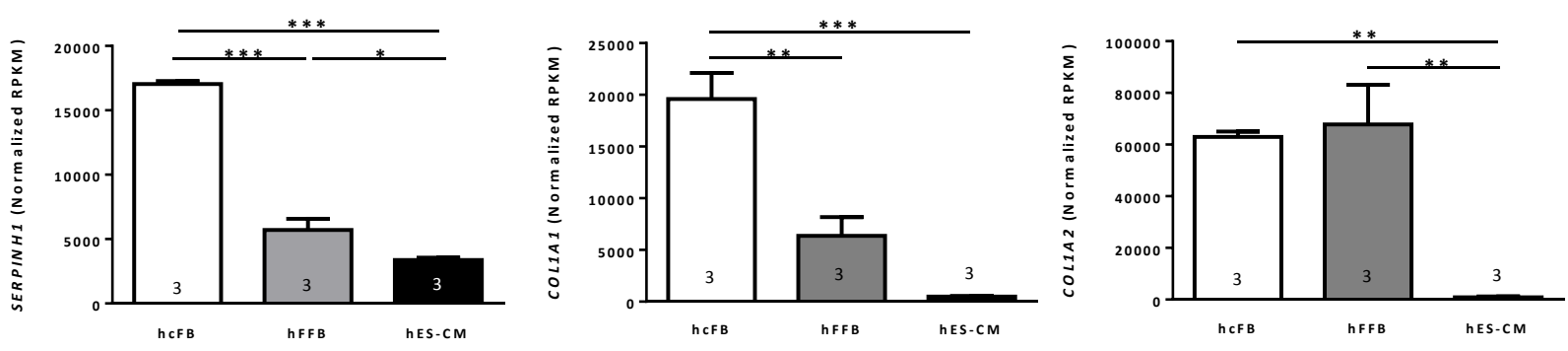

Figure 22. Collagen processing HSP47 is highly expressed in human FBs compared to CMs. (A) Western blot analyses reveals greater expression of HSP47 in human fibroblast populations (hcFBs and hFFBs) compared to purely enriched hES2-CMs (n=3/group; *P<0.05; 1-way ANOVA with Bonferroni post-hoc test). 'L': Protein molecular weight ladder. (B) Immunostaining analysis revealed strong HSP47 expression patterns (red) localized in the cytoplasm of small contaminating non-myocyte fractions from enriched CM populations. (C) RNA-seq analysis from purer CM populations revealed high SERPINH1 transcript levels in hcFBs compared to hES2-CMs. Collagen-related transcripts COL1A1 and COL1A2 showed negligable expression in hES-CMs (n=3/group; *P<0.05; 1-way ANOVA with Bonferroni post-hoc test). 
Taken together, this data indicate that collagen-synthesizing cFBs express higher levels of HSP47 than CMs in both mouse and human cells.

\subsubsection{Characterization of HSP47 KO MEFs}

To investigate the influence of collagen type I organisation on CM maturation, we utilized collagen defective HSP47 KO MEFs which were obtained from the lab of Prof. Kazuhiro Nagata in Japan (Nagai et al., 2001; Ishida et al., 2006), to study how the absence of HSP47 can influence the collagen secretion/deposition, and how this can directly influence CM maturation in both 2D culture and in our 3D EHM model of heart muscle development.

HSP47 KO MEFs were originally obtained from HSP47 KO mice. The HSP47 gene was disrupted in mice using homologous recombination, with a targeting vector deleting exon IV and part of exon $\mathrm{V}$ of the gene, and simultaneously inserting a neomycin resistance gene (as described in Nagai et al., 2001) (Figure 23A). Both RTPCR and immunoblotting analysis was initially performed to confirm the absence of HSP47 at both RNA and protein level in these MEFs. Firstly, primers targeting part of exon III to exon V of the Hsp47 gene were used for the RT-PCR assay (Figure 23A). The absence of an amplified PCR product band in HSP47 KO MEFs (+) confirmed the disruption of the Hsp47 gene compared to 'WT' MEFs at mRNA level (Figure 23B). The No RT controls (-) demonstrates that no contaminating genomic DNA was present in samples. Gapdh and $\beta$-actin housekeeper genes were expressed in both cell lines. 
A
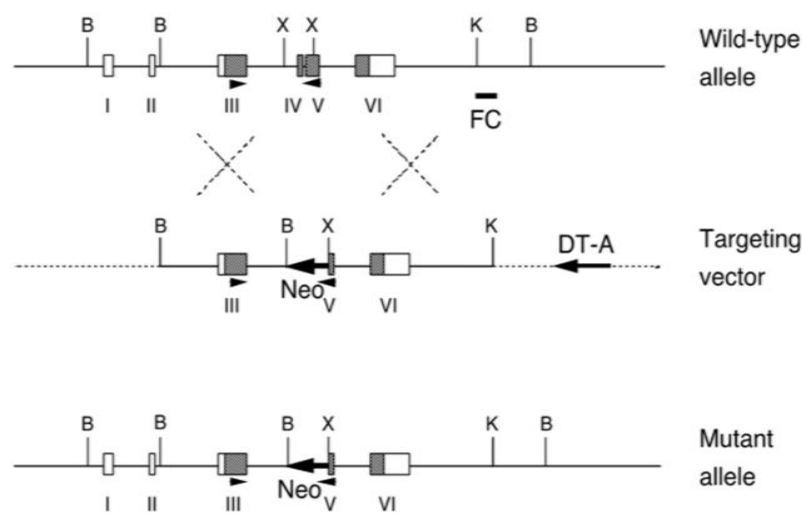

B
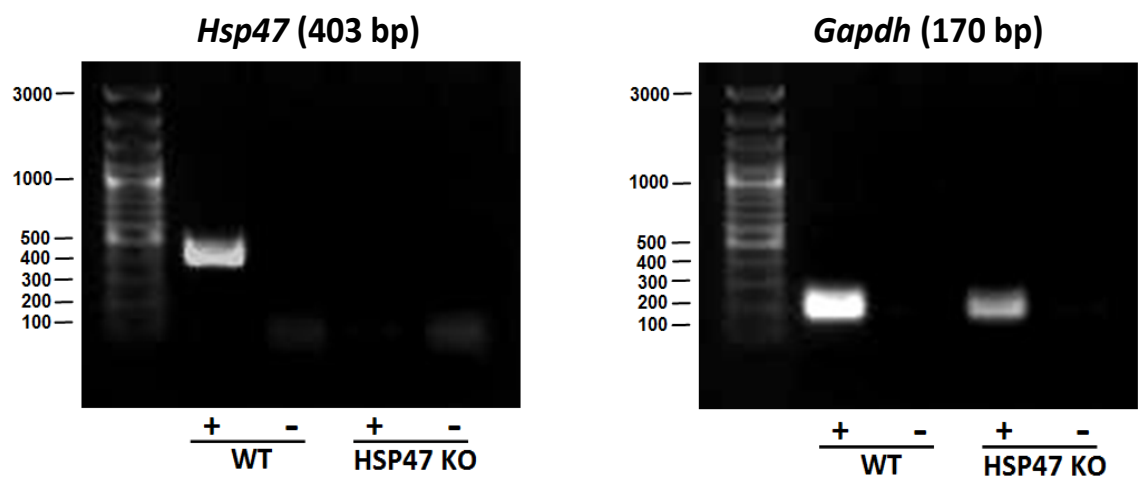

Figure 23. Confirmation of Hsp47 gene targeted disruption in HSP47 KO MEFs. (A) Homologous recombination with targeting vectors deleting exon IV and part of exon $\mathrm{V}$, and simultaneously inserting a Neo gene. Arrows indicate the orientation of the Neo gene and DT-A cassettes. Arrowheads targeting part of exon III and exon V indicate the location of primers used for RT-PCR analysis (Image taken from Nagai et al., 2000). (B) RT-PCR analysis confirmed the absence of $H s p 47$ expression at mRNA level in HSP47 KO MEFs compared to WT MEFs, using the primers denoted above. Housekeeping genes; Gapdh and $\beta$-actin were present in both WT- and HSP47 KO- MEFs. '+' indicates cDNA synthesis in the presence of reverse transcriptase, and '-'in the absence of reverse transcriptase.

Next we wanted to confirm the absence of HSP47 at protein level via immunoblotting analysis. Indeed, HSP47 expression was completely absent in the HSP47 KO MEFs, compared to WT (Figure 24A). In addition, fluorescent staining clearly showed the absence of HSP47 expression in HSP47 KO MEFs compared to WT, where there is a strong expression pattern of HSP47 (red) localized in the cellular cytoplasm surrounding the nucleus (Figure 24B). In addition, Procollagen 1 (ProCol1 $\alpha 1$ ) (green) expression within WT cells colocalizes in particular regions where HSP47 is 
expressed. Interestingly, the intracellular ProCol1 $\alpha 1$ expression in HSP47KO MEFs revealed to be very strongly expressed at particular intracellular regions near the ER, in contrast to WT MEFs in which ProCol1 $\alpha 1$ displayed a fairly more homogeneously distributed expression pattern throughout the cells. These observations suggest that ProColla1 in HSP47 KO MEFs is being aggregated and retained within the ER of these cells in the absence of HSP47.

A

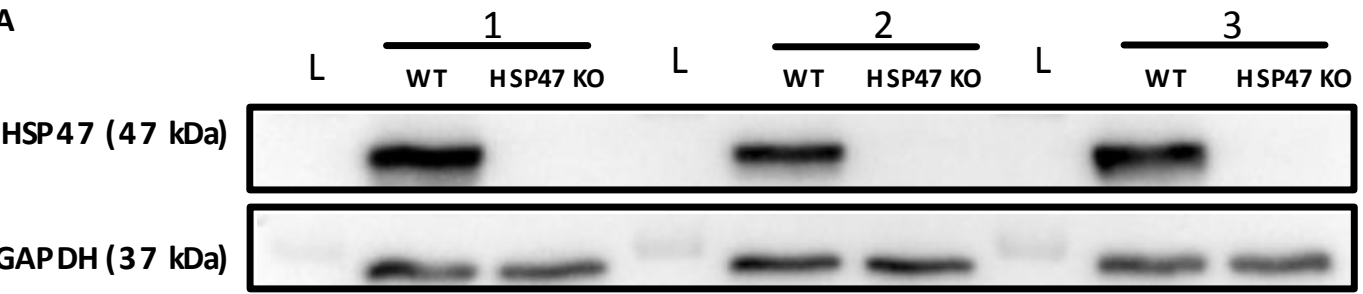

WT

B

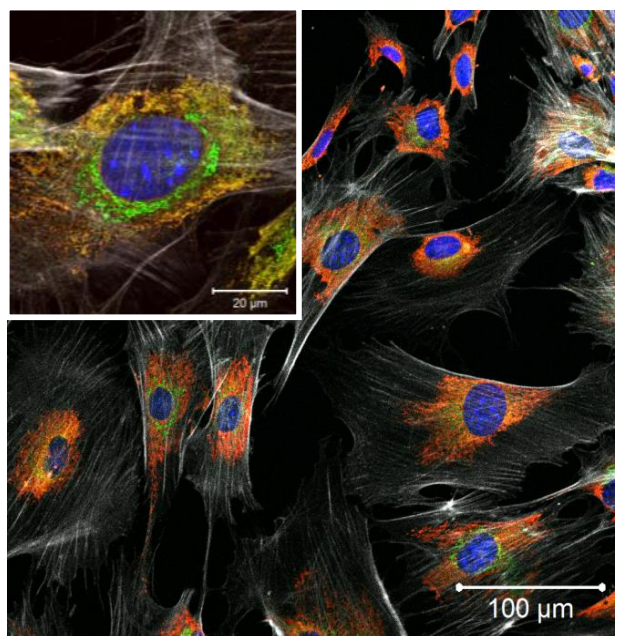

HSP47, ProCol1a1, F-actin, Hoechst

C

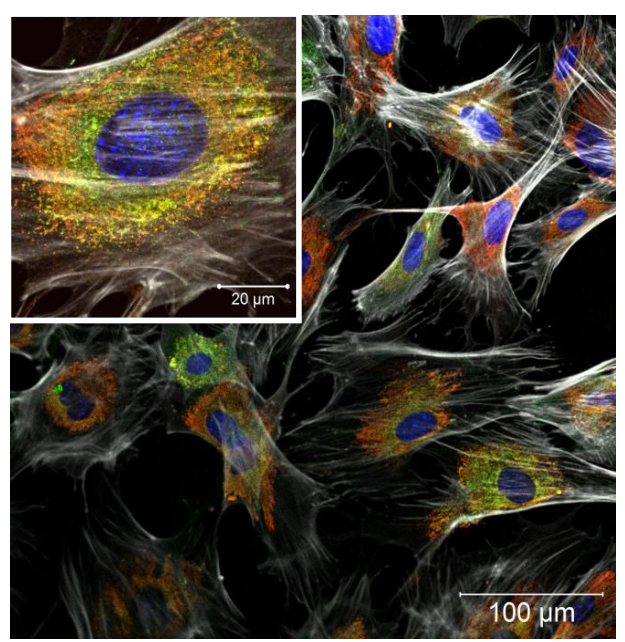

HSP47 KO
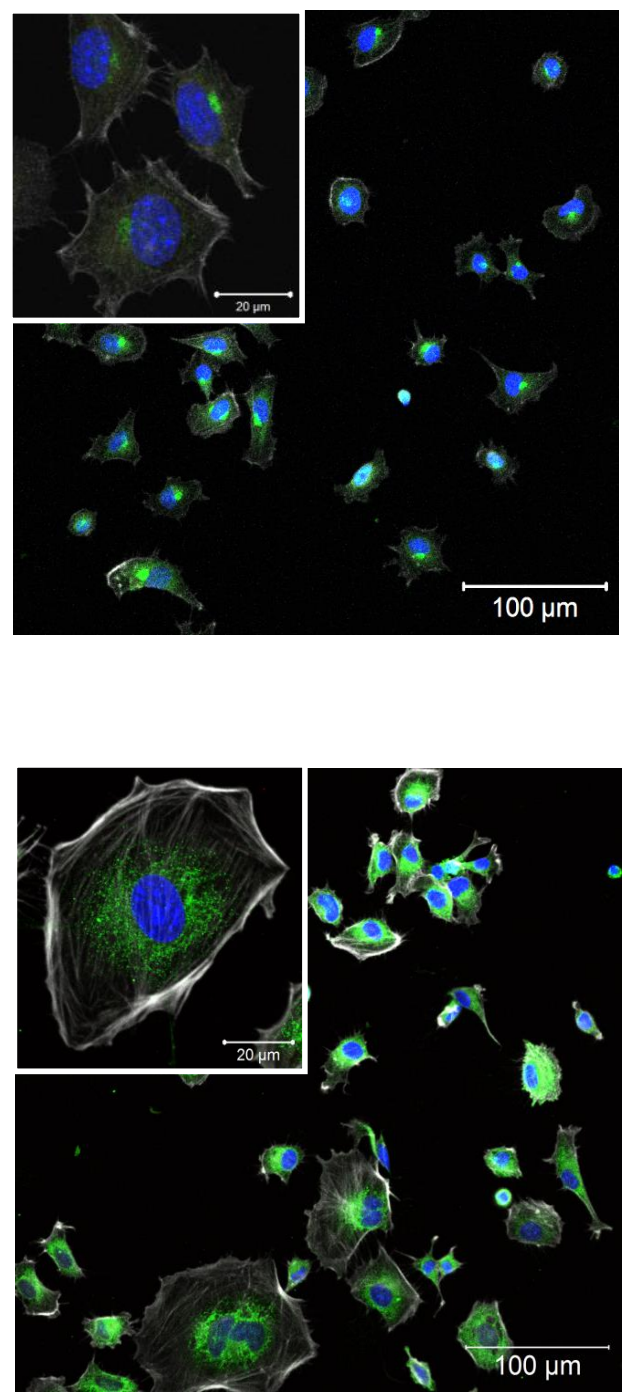

HSP47, PDI, F-actin, Hoechst 
D
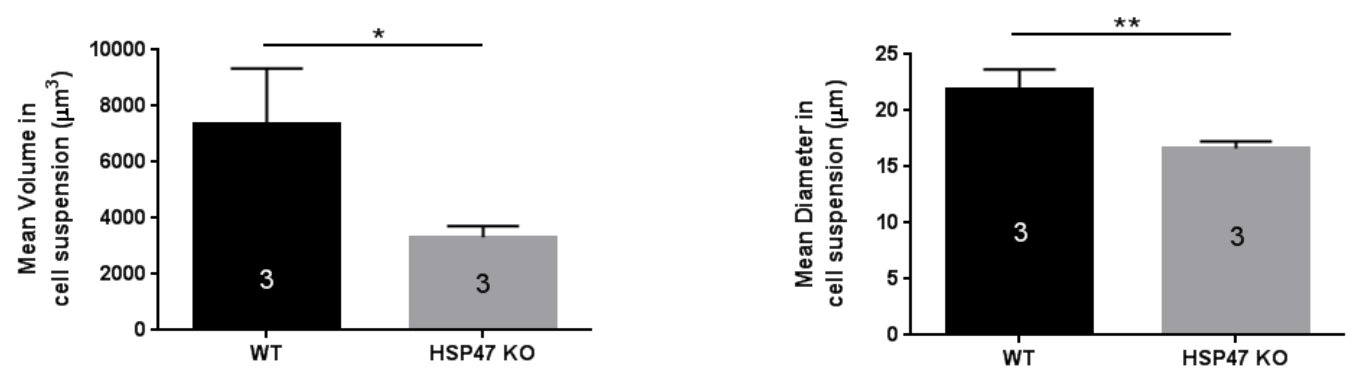

Figure 24. Absence of ER localized HSP47 at the protein level in HSP47 KO MEFs. (A) Immunoblotting analysis confirmed the absence of HSP47 protein in HSP47 KO MEFs, in contrast to WT MEFs (n=3/group). 'L': Protein molecular weight ladder. (B) ProCol1 $\alpha 1$ (green) appeared as dense aggregates in HSP47 KO MEFs, in contrast to it's more uniformly distribution in WT MEFs. (C) HSP47 expression in WT MEFs (red) colocalizes with PDI (green) in the ER. (D) CASY analysis from cell suspensions revealed that HSP47 KO MEFs were significantly smaller in cell diameter $(\mu \mathrm{m})$ and volume $\left(\mu \mathrm{m}^{3}\right)$ compared to WT MEFs $\left({ }^{*} \mathrm{P}<0.05 ; \mathrm{n}=3\right.$ measurements/group; Student's unpaired t-test).

Immunoflourescence staining furthermore revealed that HSP47 colocalizes with the protein disulphide-isomerase (PDI) (Figure 24C), which is another ER resident chaperone protein (Wang et al., 1998; Gilbert, 1997) involved in procollagen synthesis (Gelse et al., 2003; Canty et al., 2005). Interestingly, HSP47 appeared to localize exclusively at the periphery of PDI. These data collectively suggest that HSP47 expression is localized predominantly in the ER. HSP47KO MEFs furthermore exhibit both significantly smaller cell diameter and cell volume compared to WT MEFs ( $* \mathrm{P}<0.05 ; \mathrm{n}=3 /$ group) (Figure $24 \mathrm{D})$.

As we observed dense intracellular ProCol1 $\alpha 1$ aggregates in HSP47KO MEFs, we next wanted to examine whether the expression of other ER localized chaperones were influenced as a consequence for HSP47 deletion in HSP47 KO MEFs. We identified that PDI revealed a trend towards increased protein expression via immunoblotting analysis ( $n=3 /$ group), however this increase was not significant compared to WT (Figure 25). 

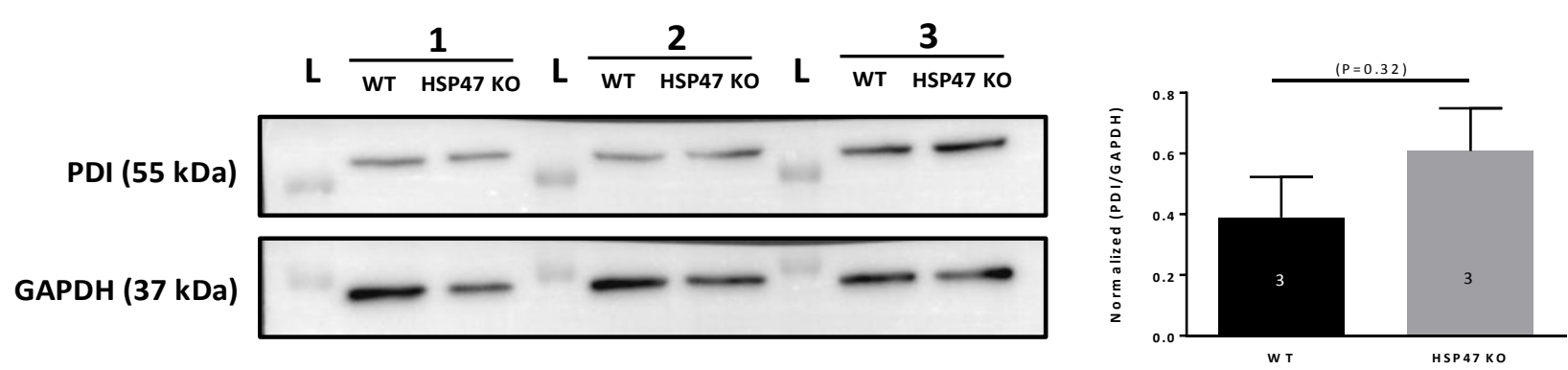

Figure 25. Expression of ER-resident chaperone PDI in HSP47 KO MEFs. Immunoblotting analysis demonstrates that PDI expression showed a trend towards increased expression in HSP47 KO MEFs ( $\mathrm{n}=3$ /group; ${ }^{*} \mathrm{P}<0.05$; Student's unpaired t-test). 'L': Protein molecular weight ladder.

\subsubsection{Defective Collagen type I secretion in HSP47 KO MEFs}

HSP47 KO MEFs have previously been shown to display slower secretion rates of collagen type I and IV (Ishida et al., 2006; Marutani et al., 2004) leading to the intracellular accumulation of procollagen. In addition, these collagens secreted from HSP47 KO MEFs were sensitive to trypsin/chymotrypsin digestion (Ishida et al., 2006; Marutani et al., 2004), which collectively suggests that procollagens were not correctly folded into triple helix structures within the ER.

From our studies, we firstly wanted to also observe whether HSP47 KO MEFs display a defective secretion rate of collagen compared to WT MEFs. Both, WT and HSP47 KO MEFs were cultured in low serum containing medium on 6 well plates, at an equal seeding density per well (Figure 26A). The conditioned medium was collected after 72 hrs. Thereafter, the amount of soluble collagen secreted into the conditioned medium was analysed using the Sircol $^{\mathrm{TM}}$ assay. In addition, plates were fixed and stained for nuclei (Hoechst). The total cell number/well could then be evaluated using a high throughput automated imaging system (Cellavista). HSP47 KO cells showed significantly reduced secreted collagen $(\mathrm{ng})$ per cell $(* \mathrm{P}<0.05 ; \mathrm{n}=5$ experiments/group) after $72 \mathrm{hrs}$ in contrast to WT cells (Figure 26B), supporting previous finding that these cells exhibit retarded collagen secretion rates. 
A

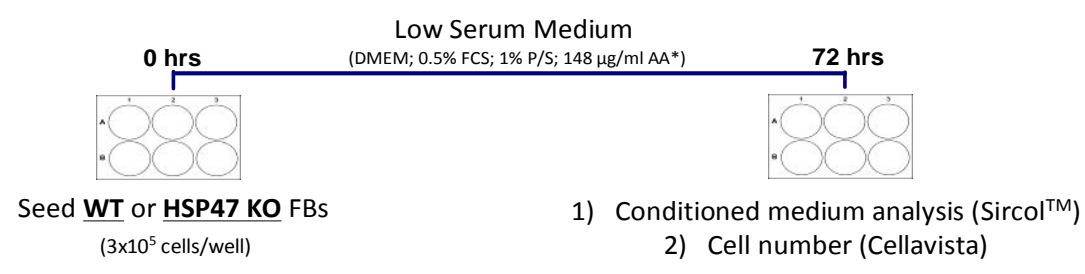

B

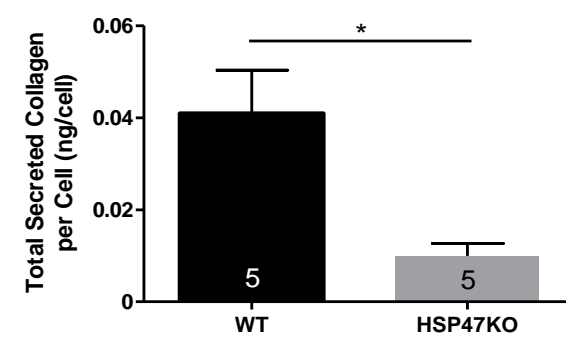

Figure 26. Absence of HSP47 expression in MEFs reduces secretion of soluble collagen. (A) WT and HSP47 KO MEFs were plated at fixed seeding densities ( $3 \times 10^{5}$ cells/well) on 6 well plates and cultivated for $72 \mathrm{hrs}$ in low serum-containing medium (AA: $148 \mu \mathrm{g} / \mathrm{ml} \mathrm{L-Ascorbic} \mathrm{Acid} \mathrm{2-phosphate}$ magnesium salt). Condition medium was subsequently collected from each well and secreted soluble collagen levels were assessed by the Sircol ${ }^{\mathrm{TM}}$ assay. Cells were then fixed and stained with Hoechst. Total cell numbers in each well were analyzed using the Cellavista platform. (B) Soluble collagen levels were normalized to cell number. HSP47 KO MEFs display markedly lower levels of soluble secreted collagen in the conditioned medium, in contrast to WT MEFs $\left({ }^{*} \mathrm{P}<0.05 ; \mathrm{n}=5\right.$; Student's unpaired t-test).

\subsubsection{Deoxychelate (DOC)-separation of ECM fractions}

In order to assess ECM components secreted from WT and HSP47KO MEFs, DOC extraction was used to separate cellular material and components not incorporated into the ECM (DOC soluble fraction) from insoluble material containing ECM (DOCinsoluble fraction; Van Duyn Graham et al., 2010; Midwood et al., 2002). DOCinsoluble ECM fractions were obtained from WT and HSP47KO MEFs seeded at equal high density and cultured for 7 days (Figure 27A). These isolated fractions were subsequently solubilised and analysed by SDS-PAGE and coomassie blue staining (Figure 27B). 
A

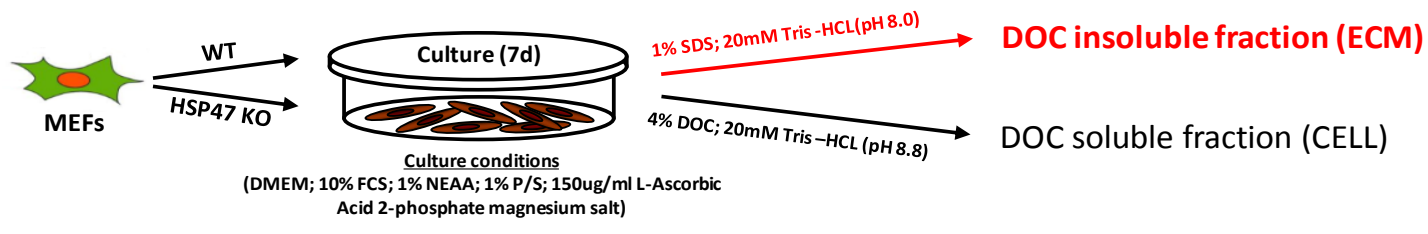

B

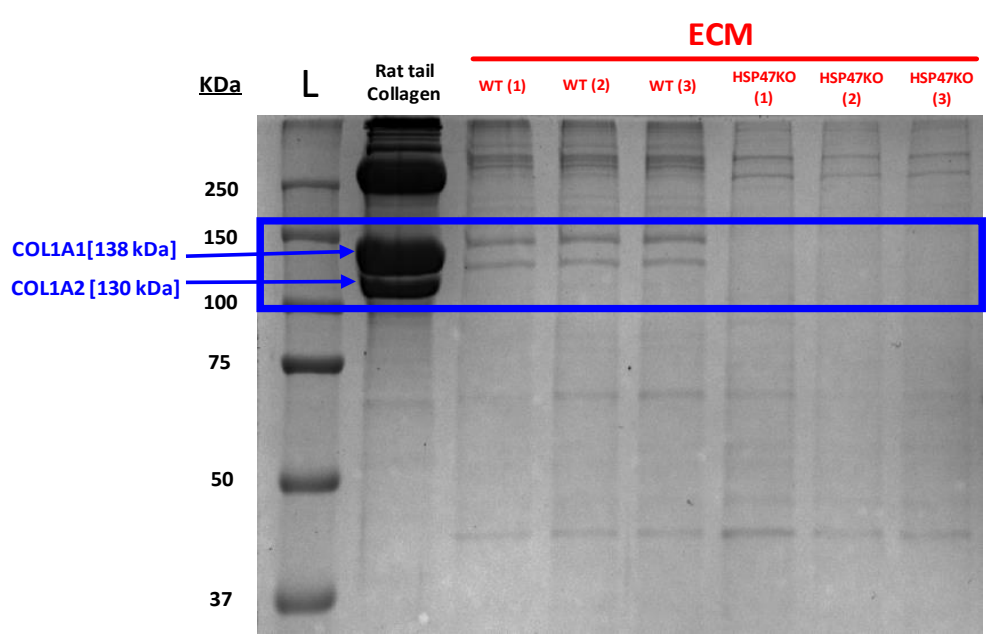

C

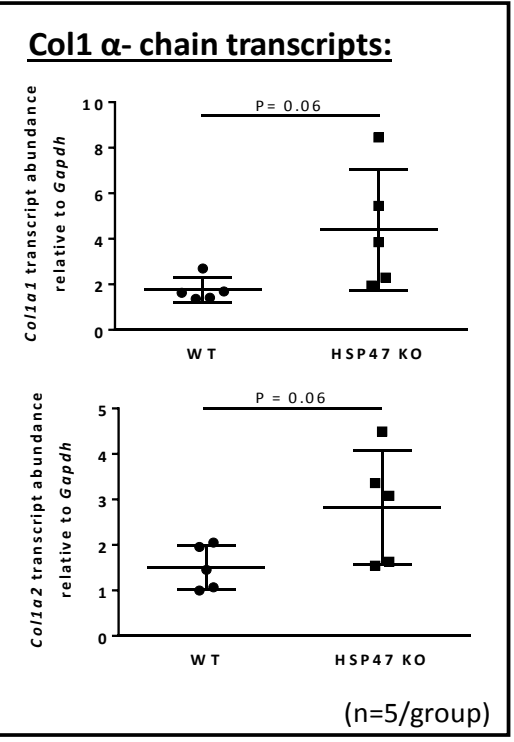

Figure 27. Reduced collagen type I protein incorporation into the ECM in HSP47 KO MEFs. (A) WT and HSP47 KO MEFs were plated at equal high seeding densities and cultured for 7 days. DOC was used to separate the cellular fractions (DOC-soluble) from the ECM fraction (DOC-insoluble). DOC-insoluble fractions were solubilised and were separated by SDS-PAGE. (B) Proteins incorporated into the ECM of WT and HSP47 KO MEFs showed distinctive profiles on Coomassie blue stained gels ( $n=3$ /group). ECM samples were compared to monomeric rat tail Collagen type I. Collagen type I chains; COL1A1 (138 kDa) and COL1A2 (130 kDa) were visibly expressed in WT-derived ECMs, in contrast to HSP47 KO-derived ECMs. 'L': Protein molecular weight ladder. (C) qPCR analysis suggested enhanced Collal and Colla2 transcript abundance in HSP47 KO MEFs (n=5/group; P<0.05; student's unpaired t-test).

Interestingly, proteins incorporated into the ECM in both WT- and HSP47 KO MEFs showed distinctive protein profiles. HSP47 KO MEFs revealed that collagen type I chains COL1A1 (138 KDa) and COL1A2 (130 KDa) showed negligible expression compared to WT MEFs, indicating that Procollagen type I failed to be incorporated into the ECM from HSP47KO FBs. qPCR analysis comparing Collal and Colla2 transcript levels between WT and HSP47KO MEFs revealed that Collagen type I transcripts showed a trend towards higher levels in HSP47 KO than WT MEFs 
(Colla1, $\mathrm{P}=0.06$; Colla2, $\mathrm{P}=0.06$ ) suggesting a compensatory mechanism (Figure 27C). Taken together, these data suggests that Procollagen type I synthesized in HSP47 KO MEFs exhibit reduced secretion and incorporation into the ECM.

\subsubsection{Mass spectrometric analyses of ECM fractions}

Mass spectrometric analyses were performed to identify other proteins in ECM (DOC-insoluble) fractions derived from WT and HSP47 KO MEFs ( $\mathrm{n}=1$; with 2 technical replicates). In total, 304 proteins were identified by nanoLC-MS/MS (Figure 28). Furthermore, 2 differentially expressed proteins; Periostin (POSTN) and Collagen 6 alpha-chain 2 (COL6A2) were exclusively expressed in WT ECM fractions, in contrast to 19 proteins (predominantly extracellular vesicular exosomerelated) expressed only in HSP47 KO ECM fractions. Its has recently been suggested that exosomes may have a functional role in selectively secreting harmful/damaged proteins, in order to alleviate intracellular stress conditions and preserve intracellular protein homeostasis (Baixauli et al., 2014). POSTN has been shown to promote proliferation and myofibroblast differentiation of FBs (Crawford et al., 2015). Furthermore, POSTN has been implicated in the regulation of collagen type I fibrillogenesis and organization (Norris et al., 2007). We also identified ECM laminin subunit proteins: Laminin subunit alpha 5 (LAMA5) and laminin subunit gamma 1 (F8VQJ3 or LAMC1) to be exclusively expressed in HSP47 KO ECM fractions.

In agreement with the data presented before (Figure 27B) collagen type I protein chains; COL1A1 (138 kDa) and COL1A2 (130 kDa) were significantly lower in protein abundance $(\mathrm{P}<0.01)$ in HSP47 KO- versus WT-ECM fractions that there is markedly reduced collagen type I incorporation into the ECM in HSP47KO MEFs. Moreover, collagen type $\mathrm{V}$ proteins (COL5A1, COL5A2), collagen type VI proteins (COL6A2) and Keratin, type II cytoskeletal 1 (K2C1) were of higher abundance in WT ECM fractions. On the other hand; 2 vesicular exosome proteins Heat Shock Protein 90-beta (HSP90B) Endoplasmin (ENPL) were identified to be higher expressed in HSP47 KO MEFs derived ECM $(\mathrm{P}<0.01)$. 


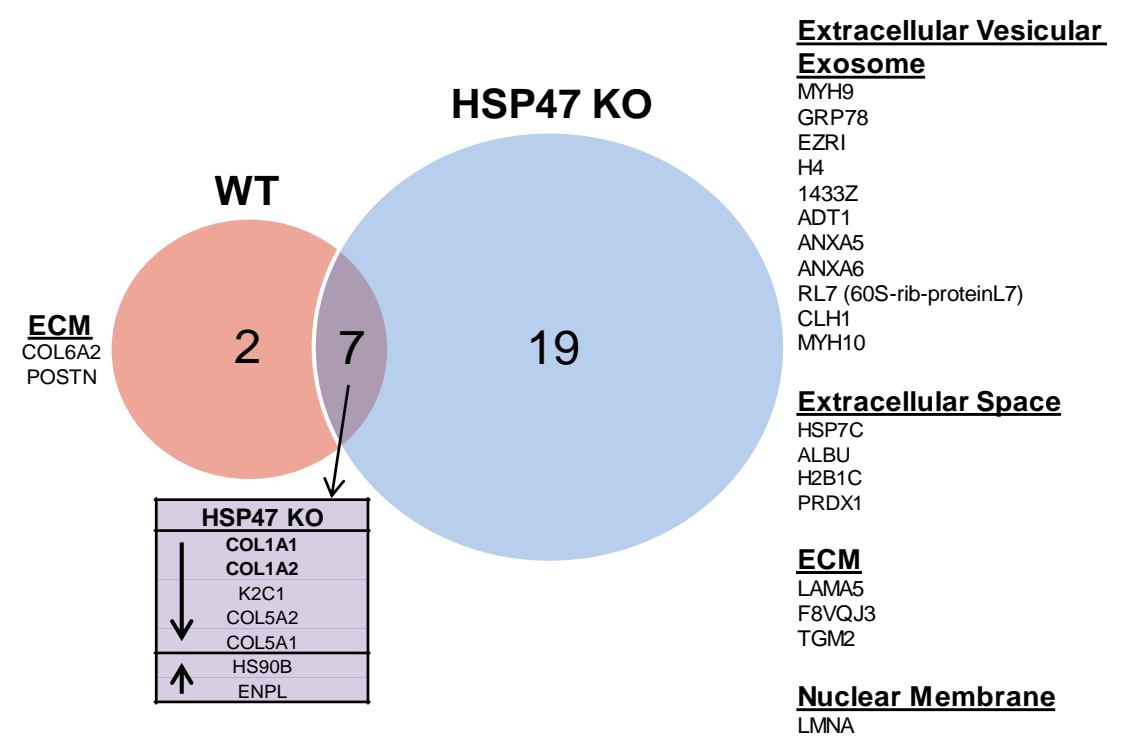

Figure 28. Venn diagram illustrating the number of proteins identified in ECM fractions from WT- and HSP47 KO MEFs. Proteins identified by mass spectrometry analysis were found to be exclusively expressed in the respective WT or HSP47KO ECM (DOC-insoluble) fractions, or significantly upregulated/downregulated in HSP47 KO ECM (purple box) $\left({ }^{*} \mathrm{P}<0.01 ; n=1 /\right.$ group; 2 technical replicates). 19 proteins were identified to be exclusively expressed in the HSP47KO ECM fraction, in contrast to 2 proteins expressed exclusively in WT ECM fractions. Collagen type I chains (COL1A1 and COL1A2) expression was significantly less abundant $(\mathrm{P}<0.01)$ in the HSP47 KO ECM fraction, compared to WT.

Taking together, this data suggests that HSP47 KO MEFs exhibit ECM dysregulation, as shown by the reduced expressions levels of collagens and incorporation of collagen type I into the ECM, compared to WT MEFs.

\subsubsection{Investigation of cardio-instructive properties of fibroblast-derived ECM}

It has previously been shown that embryonic cFBs develop coinciding with expansion of the ventricular compact layer and regulate proliferation of immature CMs via secretion of growth factors the ECM components (Ieda et al., 2009). We specifically asked whether the FB-derived ECM environment has an impact not only on proliferation but also in maturation of CMs. Furthermore, since our study suggested collagen defective HSP47 KO MEFs display reduced collagen secretion (Figure 26) and reduced collagen type I incorporation into the ECM (Figure 27 and 28), we wanted to determine whether the ECM produced from collagen defective HSP47 KO MEFs can impact the cardio-instructive properties of CMs. 


\subsubsection{Generation of fibroblast-derived ECM}

We established a FB-derived ECM model by growing MEFs at high density on culture plates for 11 days in serum containing medium containing; $148 \mu \mathrm{g} / \mathrm{ml} \mathrm{L}$ Ascorbic Acid 2-phosphate magnesium salt, to facilitate the deposition of a dense ECM network. Thereafter, the ECMs were denuded of FBs and cellular remnants were removed via DNase I treatment (Figure 29A). This extraction procedure resulted in an intact FB-derived ECM that was free of cellular debris. Immunostaining analysis of the decellularized FB-derived ECM revealed a dense fibrous mesh-like ECM network deposited on the bottom of plates, which comprised of dense collagen type I fibrillar ECM (red) (Figure 29B). In addition, ECMs were free of nuclei (blue) and cellular cytoskeletal actin (green) indicating complete cellular lysis, compared to before treatment.

A

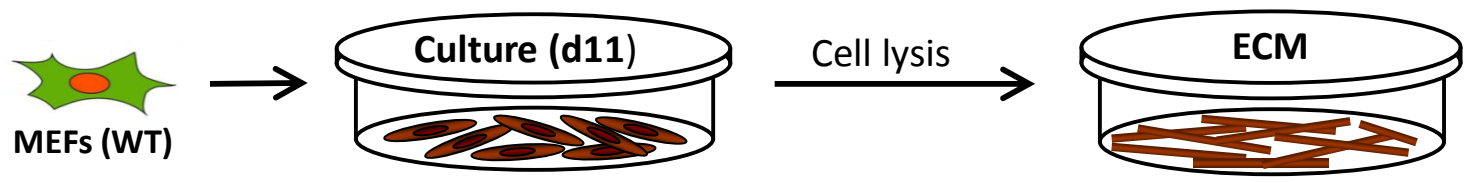

BEFORE

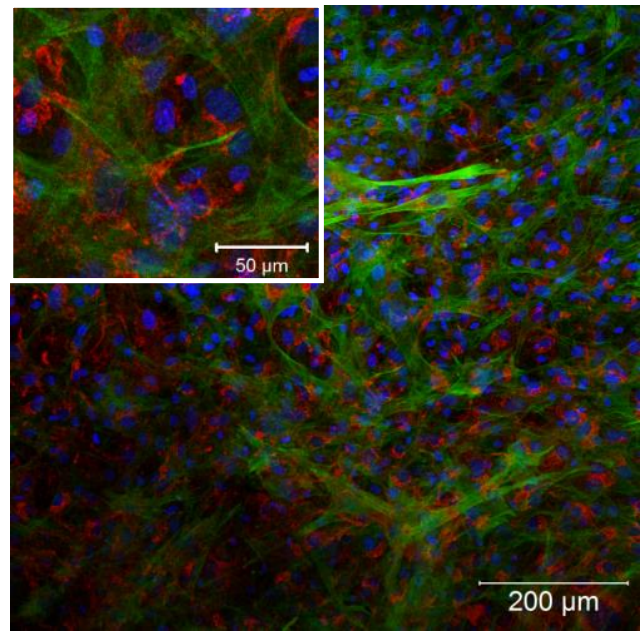

AFTER

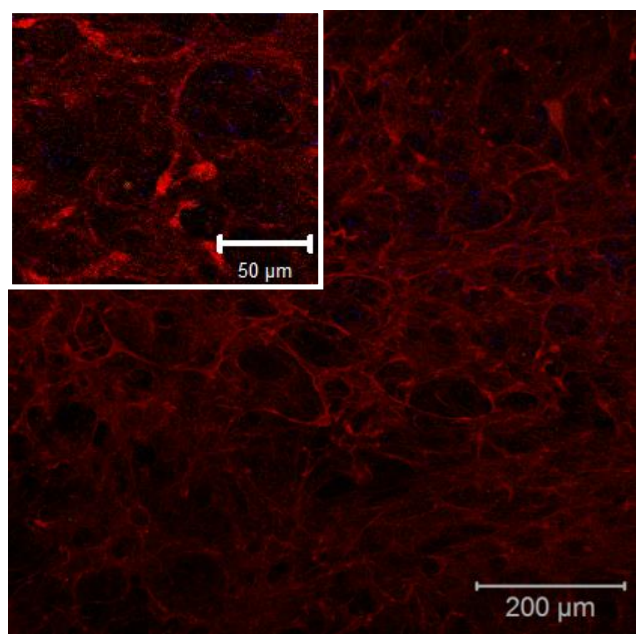

Collagen 1, F-actin, Hoechst

Figure 29. Generation of denuded FB-derived ECM before and after decellularization. (A) MEFs were plated at high seeding densities and cultured for 11 days in medium containing $148 \mu \mathrm{g} / \mathrm{ml} \mathrm{L-}$ Ascorbic Acid 2-phosphate magnesium salt. FB-derived ECMs were subsequent obtained after cell lysis (decellularization) and DNase I treatment. (B) Denuded FB-derived ECMs were free of cellular nuclei (blue) and cellular cytoskeletal actin (green) [AFTER], compared to before decellularization [BEFORE]. In addition, a dense collagen type I fibrillar ECM network (red) was deposited from the FBs. 


\subsubsection{Reduced ECM deposition and abnormal Collagen type I fibril formation in HSP47 KO MEFs}

Next, we wanted to investigate the deposition and structural organization of secreted ECM Collagen type I in collagen defective HSP47 KO MEFs (Figure 30).

A
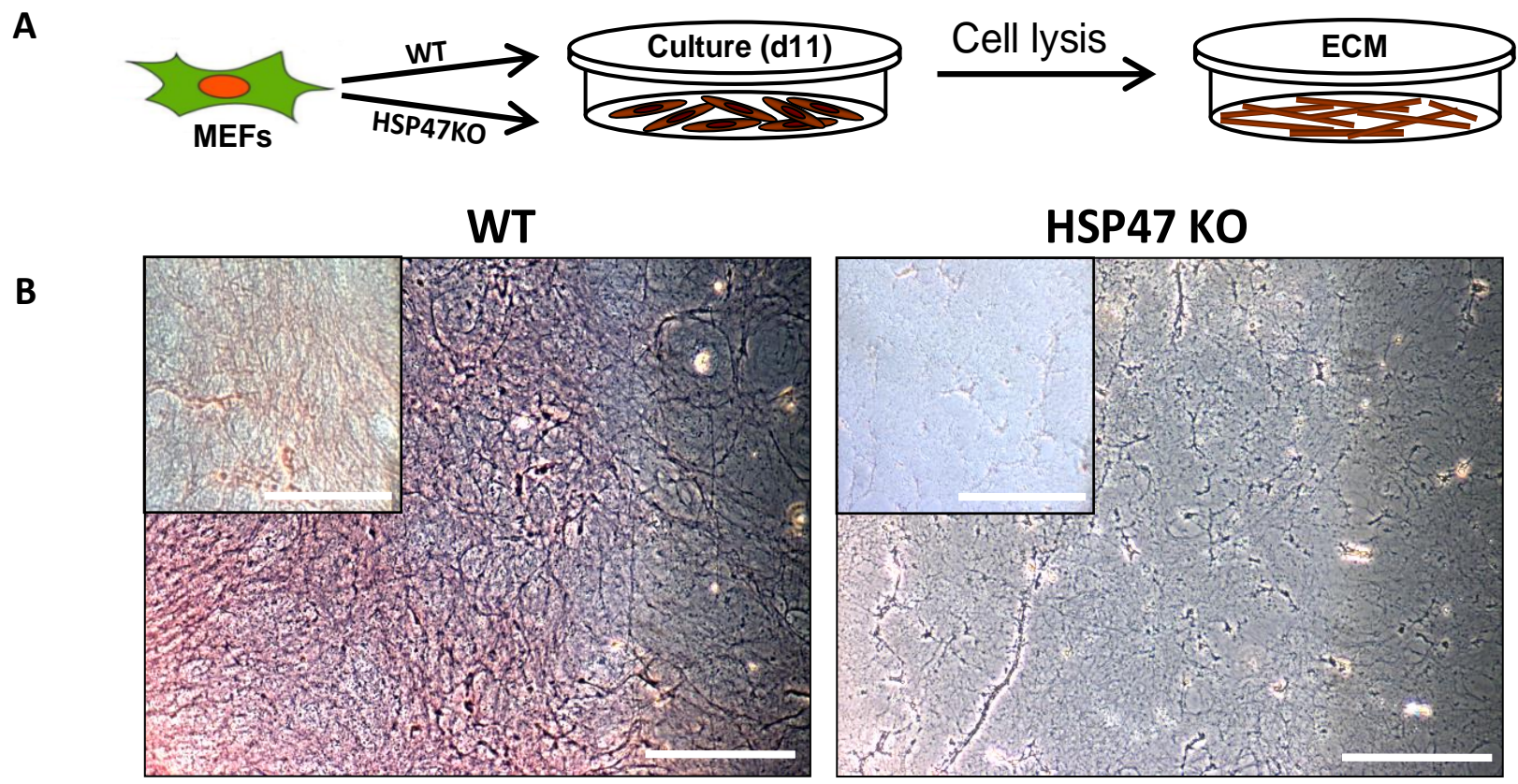

C
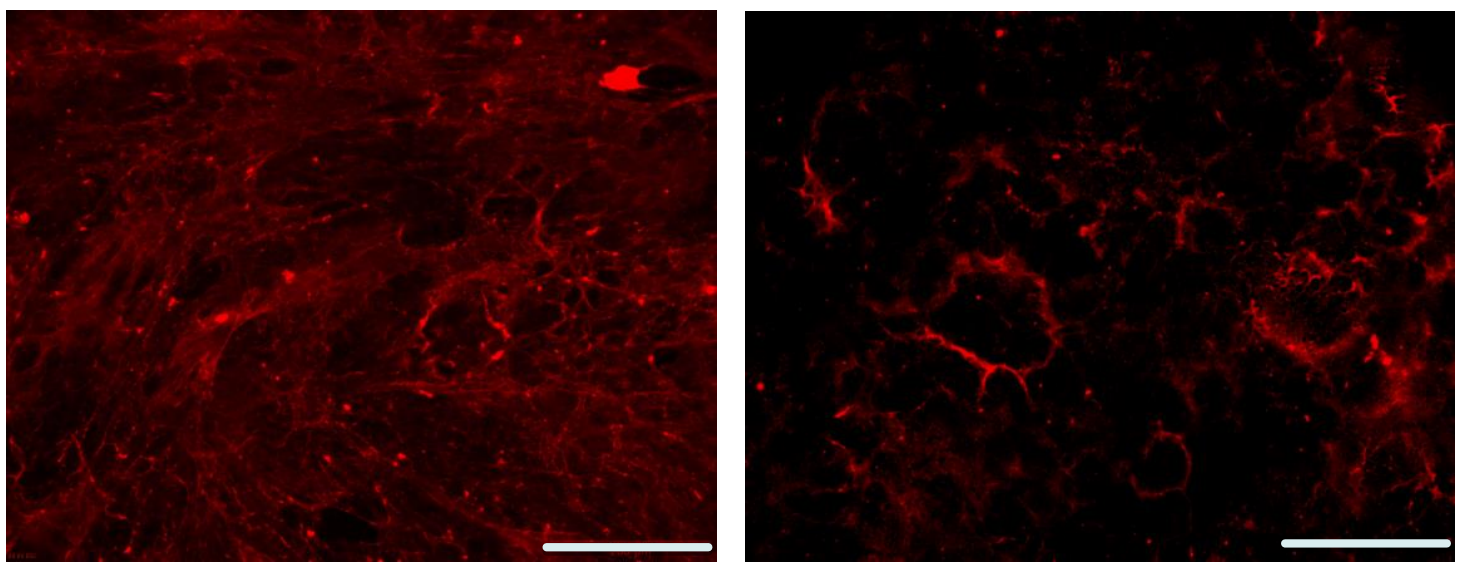

Collagen 1

Figure 30. Reduced Collagen type I fibril formation and deposition in HSP47 KO MEF-derived ECMs. (A) Schematic overview of the fabrication of FB-derived ECM from WT or HSP47 KO MEFs. (B) Staining of the total ECM proteins by Ponceau S and (C) immunoflourescence staining of Collagen type 1 (red) show dense microfibrillar network structures from WT MEF-derived ECM, in contrast to HSP47 KO MEF-derived ECM which displayed markedly reduced Collagen type 1 deposition, and both thinner and reduced fibrous ECM networks (Scale bars: $200 \mu \mathrm{m}$ ). 
Ponceau S staining (Figure 30B) and immunostaining analysis of extracellular collagen type I (Figure 30C) in FB-derived ECM deposited from HSP47 KO MEFs were sparse and weakly stained compared to the dense ECM networks observed in WT MEFs. Furthermore, longitudinally aligned collagen type I fibres were present in WT MEFs, in contrast to abnormal Collagen type I fibril formation observed from HSP47KO MEFs (Figure 30C). 


\subsubsection{Impaired cardiomyocyte phenotype on defective collagen processing HSP47 KO FB-derived ECMs}

To directly investigate whether the absence of HSP47 can influence the cardioinstructive properties of the ECM niche secreted from FBs, we cultured immature mESC-enriched CMs (mCMs) on purely FB-derived ECM from WT and HSP47 KO MEFs (Figure 31).

A

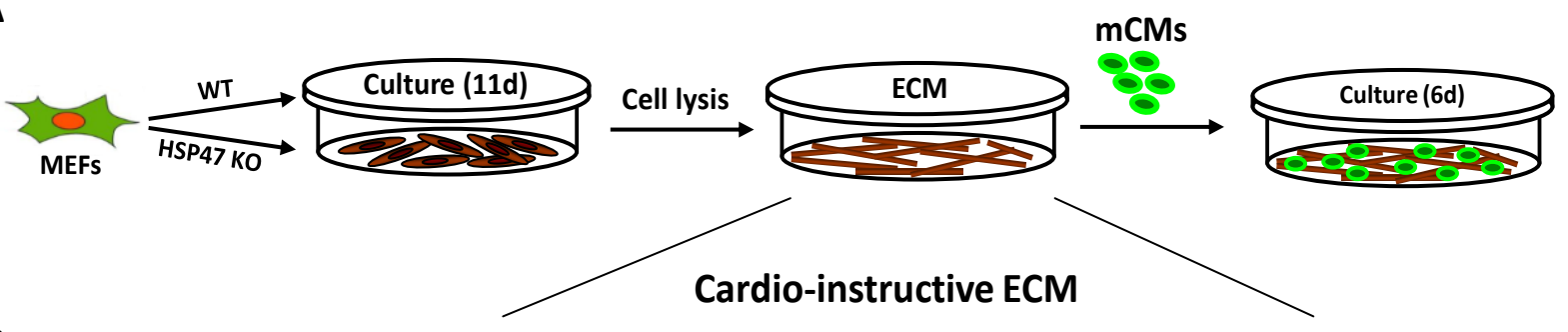

B
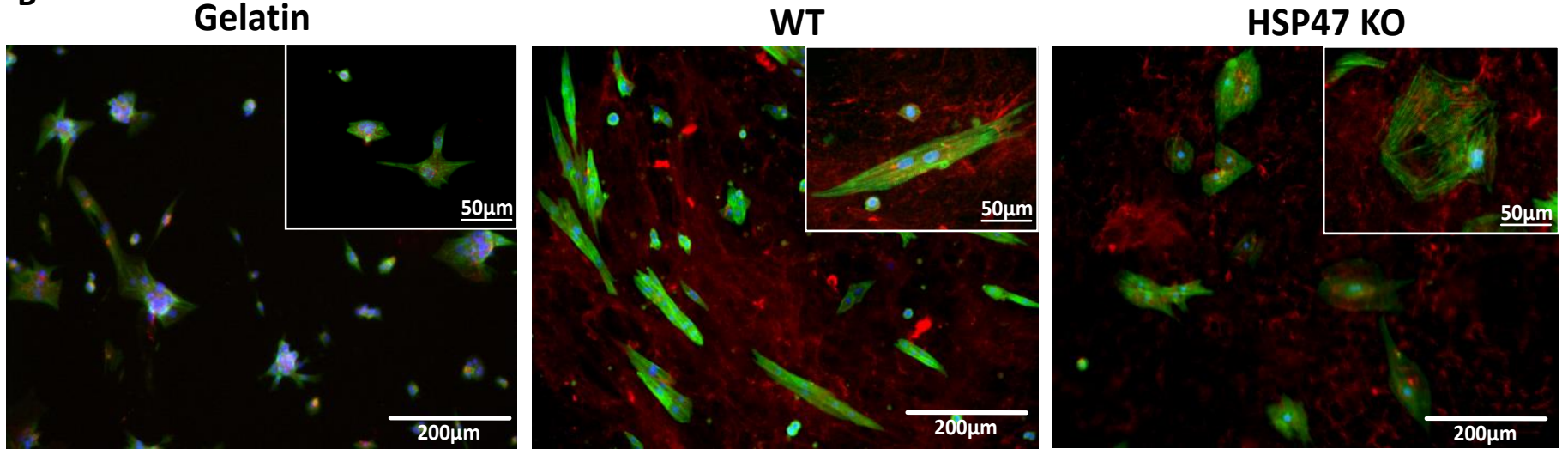

$\alpha$-actinin/Collagen $1 /$ Hoechst

C
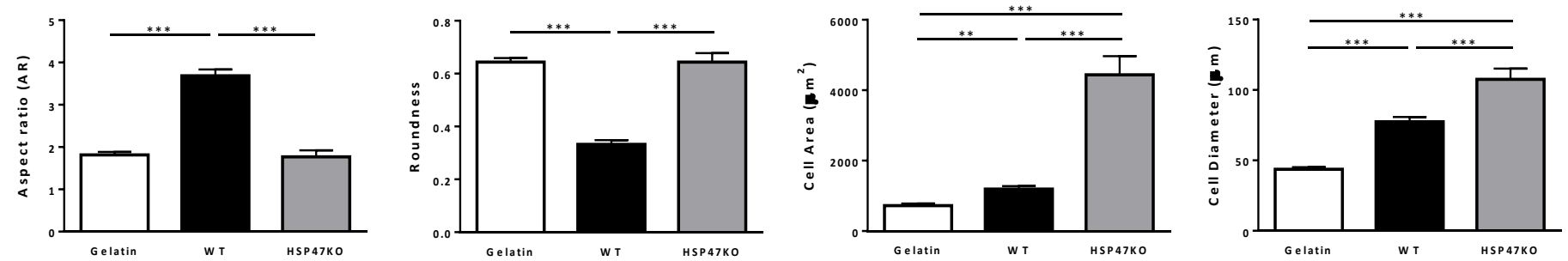

Figure 31. Impaired CM morphology on collagen defective HSP47 KO MEF-derived ECMs. (A) To assess the cardio-instructive properties of FB-derived ECMs, genetically enriched immature mCMs were cultivated for 6 days on WT- or collagen defective HSP47 KO MEF-depleted ECMs. (B) mCMs ( $\alpha$-actinin-green; Hoechstblue) showed advanced morphological features with regular cross-striations and elongated rod-shaped cell bodies on WT MEF-depleted ECMs (Collagen 1-red), in contrast to HSP47 KO-MEF-depleted ECMs and classic monolayer culture (gelatin), which displayed mainly immature morphology and disorganized sarcomeric structures. (C) Analysis of single cell morphological parameters; Aspect ratio (AR), roundness, cell area and cell perimeter. ( $\mathrm{n}=\geq 30$ cells/group; $* \mathrm{P}<0.05 ; 1$-way ANOVA with Bonferroni post-hoc test). 
Interestingly, mCMs cultured on well organized- WT MEF-derived ECM conditions displayed advanced sarcomeric organization and morphological features with regular cross striations (green) and primarily rod-shaped bodies and containing centrally localized nuclei (blue; Figure 31B). In addition, morphological analysis demonstrated that these mCMs exibit a significantly higher 'Aspect Ratio (AR)' and low 'roundness' value $(* \mathrm{P}<0.05)$, compared to when cultured on gelatin-coated classical monolayers (Figure 31C). Moreover, CMs within the ECM were orientated along the collagen type I microfibrillar structure (red). In contrast, mCMs cultured on collagen defective HSP47KO MEF-derived ECM conditions showed mainly immature morphology and disorganized sarcomeric structures. This morphology was similar to $\mathrm{mCM}$ cultured on gelatine-coated classical monolayer cultures. Morphological analysis demonstrates that mCMs did not show differences in 'AR' and 'roundness values' compared to mCMs cultured on gelatin-coated substrates (Figure 31C). In addition, these mCMs were more flat and spread out (as indicated by their large surface area) on HSP47KO MEF- ECMs. This data firstly suggests FBs provide a cardio-instructive ECM environment which is guiding advance maturation of mCMs. Secondly; improper processed Collagen type I deposited from HSP47 KO MEFs impairs the cardio-instructive properties of the ECM.

\subsubsection{Collagen defective HSP47 KO MEFs impair EHM formation and functionality}

As we have demonstrated that HSP47 KO MEFs exhibit both retarded collagen type I secretion and defective ECM cardio-instructive properties, we next wanted to investigated the role of collagen type I organisation in cardiac tissue formation and maturation, utilizing our 3D EHM model of heart muscle development.

Purified mCMs were supplemented with either WT MEFs, HSP47 KO MEFs or without FBs [mCM (only)] to generate EHMs (Figure 32A). As previously shown, EHMs comprised of mCMs only failed to initially retract and form condensed tissue after 3 days on circular casting molds (8 EHMs/group; *P<0.0001; Figure 32B). Interestingly, HSP47 KO FB-supplemented EHMs failed to form well condensed 
tissue after 3 days, in contrast to more compact WT FB-EHMs (8 EHMs/group; $* \mathrm{P}<0.0001)$. Subsequently, after 7 days of static mechanical strain, flourescent stainings revealed that HSP47 KO-EHMs showed less elongated and anisotropically organised CMs and non-myocytes ( $\alpha$-actinin-green; F-actin-red; Hoechst-blue), in contrast to beating WT-EHMs in which the mCMs formed tightly packed muscle bundles (Figure 32C). This observation was comparable to EHMs generated with mCMs (only), which failed to form a functional syncytium and were structurally immature, as indicated by the majority of round shaped mCMs. The functionality of these EHMs was assessed by a measurement of contractile forces under isometric conditions (Figure 32D). HSP47 KO MEF-supplemented EHMs failed to develop contractile forces under these conditions, in contrast to WT MEF-supplemented EHMs ( $\mathrm{n}=4$; WT-supplemented EHMs: $0.143 \pm 0.008 \mathrm{mN}$ at $2.4 \mathrm{mmol} / \mathrm{L} \mathrm{Ca}^{2+}$ ). Taken together, this data collectively suggests that collagen defective HSP47 KOMEFs failed to support tissue formation and maturation of EHMs. 
A

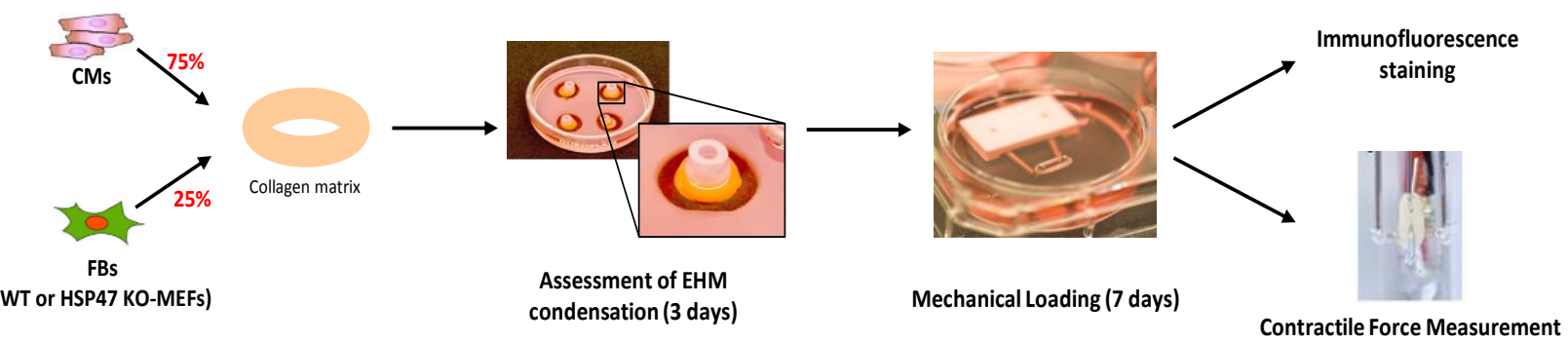

B
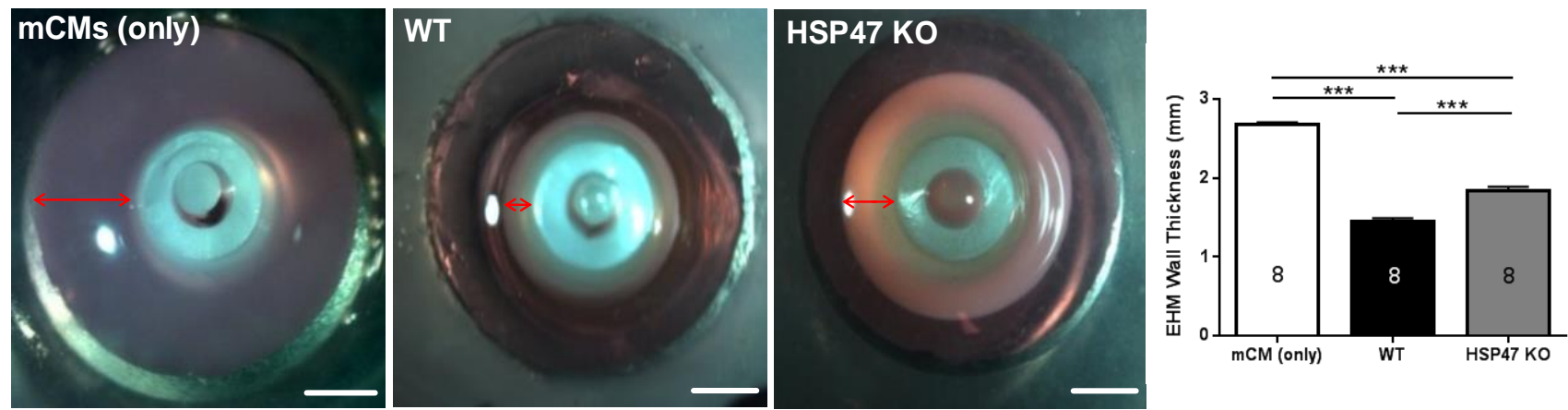

C
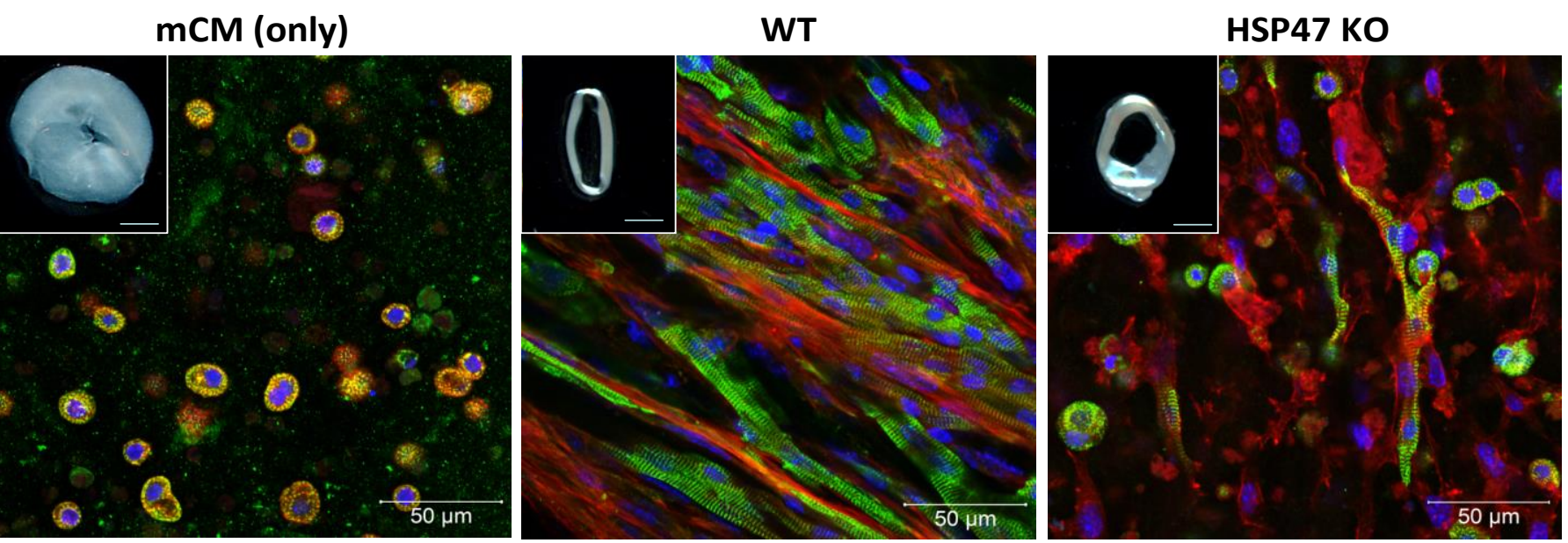

Q-actinin/F-actin/Hoechst

D

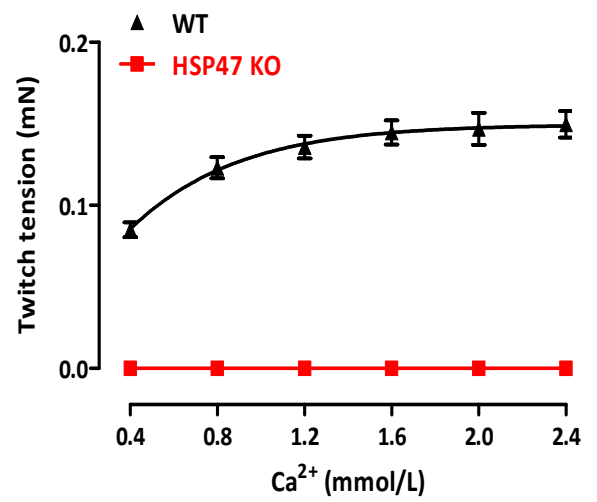


Figure 32. Immature EHMs after supplementation with HSP47 KO MEFs. (A) Schematic overview outlining the experimental details. (B) EHM 3 days after casting with: mCM (only), WTMEF and HSP47 KO-MEFs (from left to right) and red arrows indicating EHM thickness (condensation). Bar graph summarizes the data from $\mathrm{n}=8 \mathrm{EHMs} /$ group $(* \mathrm{P}<0.05 ; 1$-way ANOVA with Bonferroni post-hoc test). Scale bar: $2 \mathrm{~mm}$. (C) Immunoflourescence staining of EHM with mCM (only) and EHM supplemented with WT-MEF or HSP47 KO MEF with 10 days of culture. Inset scale bars: $2 \mathrm{~mm}$. (D) Twitch tension analysed by concentration response measurements under increasing concentrations of extracellular $\mathrm{Ca}^{2+}(\mathrm{mmol} / \mathrm{L})$. No contractile force was developed in HSP47 KO MEFsupplemented EHMs (n=4/group).

\subsubsection{Reduced Itgß1 integrin expression in HSP47 KO MEFs}

Since our studies have collectively demonstrated that ECM-integrin crosstalk from FBs is necessary for EHM formation and maturation (Figures 15, 16, 17 and 18) and we observed that HSP47 KO MEF-supplemented EHMs failed to form compact functional tissue, we thus wanted to compare the expression of the predominant collagen binding integrin receptor subunit Itg $\beta 1$, in HSP47KO- and WT-MEFs. qPCR analysis revealed that $\operatorname{Itg} 1$ transcript levels were significantly reduced in HSP47KO MEFs compared to WT MEFs $(* \mathrm{P}<0.05$; $\mathrm{n}=4-5$ /group; Figure $33 \mathrm{~A})$, however no difference was observed on protein level ( $\mathrm{n}=3$; Figure 33B).

In order to address whether HSP47 KO MEFs show reduced ECM interaction, we performed 2D cell attachment assays. Single cell suspensions of WT- or HSP47 KOMEFs were initially incubated with ITGB1 blocking antibody (CD29; $40 \mu \mathrm{g} / \mathrm{ml}$ ) for 30 mins (Figure 33C). Treated cells were then incubated for $6 \mathrm{hrs}$ on collagen type I coated plates, and subsequently counted. ITGB1 blocking of cell-collagen interactions significantly inhibited cell attachment in both WT- and HSP47 KO MEFs compared to without blocking treatment (control; Figure 33D). Blocking of cell-collagen attachment in HSP47 KO MEFs however was significantly less pronounced compared to WT MEFs which is likely due to reduced Itg $\beta 1$ expression in HSP47 KO MEFs. Unexpectedly, the levels of cell attachment between untreated WT and HSP47KO cells (control groups) after 6 hrs however did not significantly differ. 
Overall, these data confirm the importance of Itg $\beta 1$ for cell attachment and suggesting on one hand alternative mechanisms for cell attachment in the HSP47 KO group.

A

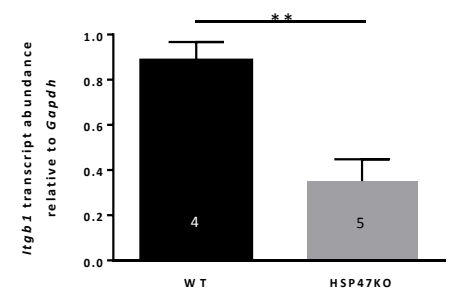

B

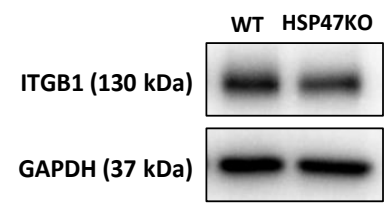

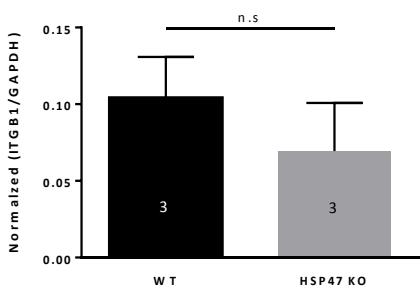

$w_{\mathrm{T}}$

C

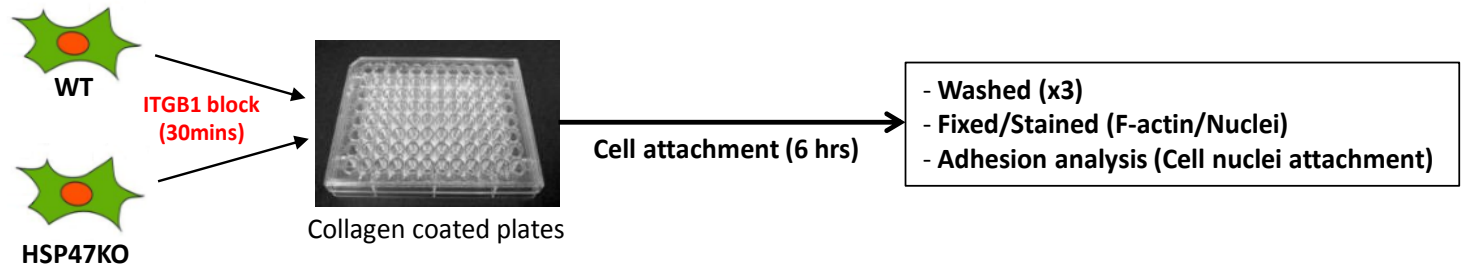

D

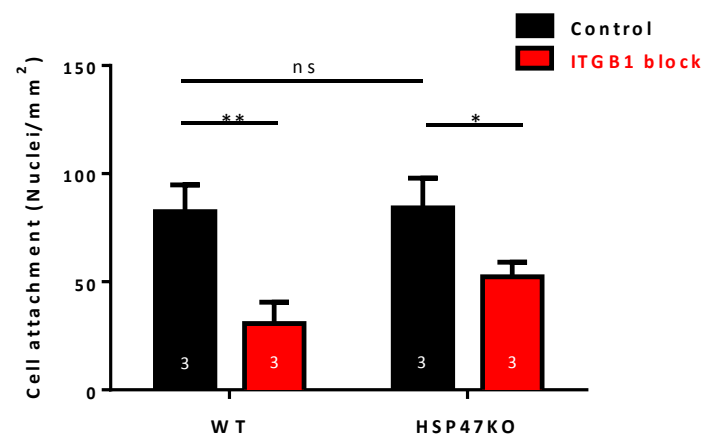

Figure 33. Reduced Itgß1 integrin expression in HSP47 KO MEFs. (A) qPCR analysis showed a significant reduction of Itgbl transcripts in HSP47 MEFs ( $\mathrm{n}=\geq 4$ /group; ${ }^{*} \mathrm{P}<0.05$; Student's unpaired ttest). (B) Immunoblotting analysis revealed a not significantly reduced expression of ITGB1 in HSP47KO MEFs, compared to WT (n=3/group). (C) Schematic diagram outlining 'integrin block cell attachment' experiments. WT and HSP47 KO MEFs were individually incubated in the absence (control) or presence of ITGB1 (CD29; $40 \mu \mathrm{g} / \mathrm{ml}$ ) blocking antibody for $30 \mathrm{mins}$, prior to being plated onto collagen-coated 96 well plates. Cells were allowed to attach for $6 \mathrm{hrs,}$, and were thereafter fixed and stained for cell count analysis $\left(32 \mathrm{~mm}^{2} /\right.$ well analyzed with $>7$ technical replicates/group in 3 independent experiments [Cellavista]). (D) WT cells treated with the ITGB1 blocking antibody (red) showed markedly reduced cell attachment ( $\mathrm{n}=3$ experiments/group; $* \mathrm{P}<0.05)$ compared to untreated cells (black). HSP47 KO MEFs also displayed reduced cell attachment with blocking treatment; however this was not as profound as compared to WT cells treated with the ITGB1 blocking antibody. 


\subsubsection{Generation and validation of HSP47 KO hES2 cell line}

As HSP47KO MEFs exhibit reduced collagen type I secretion, defective ECM cardioinstructive properties and impair mouse EHM tissue formation and maturation, we next wanted to assess whether these findings were similar in a human system. In order to investigate this, we employed the CRISPR/Cas9 system to mutate site specific regions of the SERPINH1 gene in pluropotent hES2-RFP cells (Irion et al., 2007).

Two different human mutant HSP47 KO cell lines were produced; Mut.1 and Mut.2. The Mut. 1 cell line has a 117 bp deletion of exon 3, including the genomic region of a published SERPINH1 human mutation (Christiansen et al., 2010). The Mut.2 cell line has a 200 bp deletion in exon 4-5 (start of exon 4 and start of exon 5 [90 bp of the coding DNA sequence], and the intron in between [110 bp]), similar to mouse HSP47 KO mouse model (Nagai et al., 2001). Both mutations lead to an in-frame truncation of HSP47. PCR genotyping demonstrates that both mutations were homozygous and present in the genomic DNA (Figure 34). Further experiments were additionally performed to determine whether HSP47 expression was successfully disrupted in pluropotent hES2 cells at both mRNA and protein level (Figure 35).

A

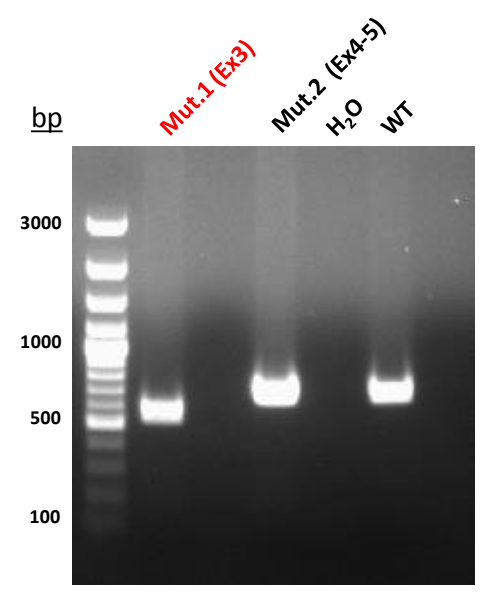

WT: $663 \mathrm{bp}$

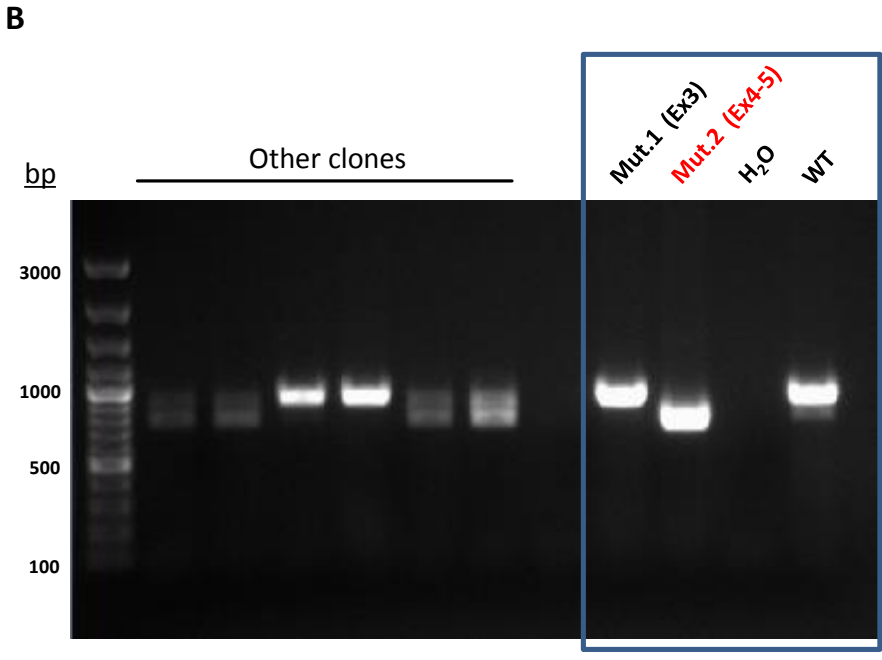

WT: 943 bp

Figure 34. PCR genotyping of SERPINH1 homozygous mutant cell lines. (A) PCR genotyping for exon 3 confirmed that the Mut.1 (exon 3 ) cell line (denoted in red) had a 117 bp deletion, compared to WT (663 bp) and Mut.2. (B) PCR for exon 4-5 confirmed that Mut.2 (exon 4-5) cell line (denoted red, in the blue box) had a 200 bp deletion, compared to WT (943 bp) and Mut.1. Both mutant lines were homozygous. Water $\left(\mathrm{H}_{2} \mathrm{O}\right)$ was used for negative controls. (Data generated by Dr. Claudia Noack). 
A

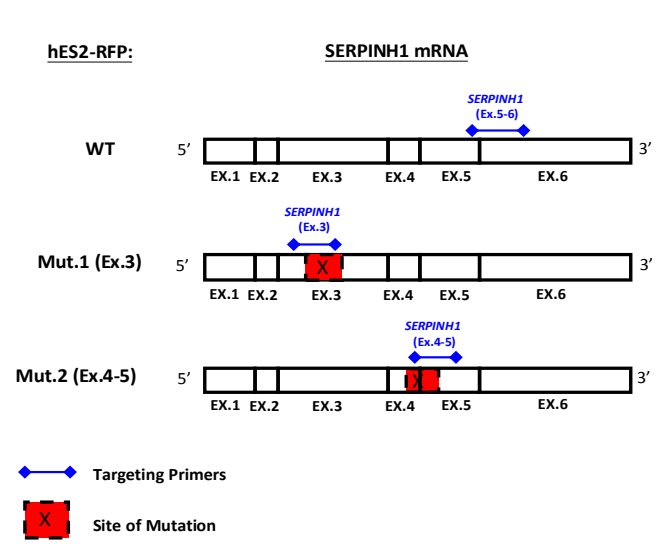

B
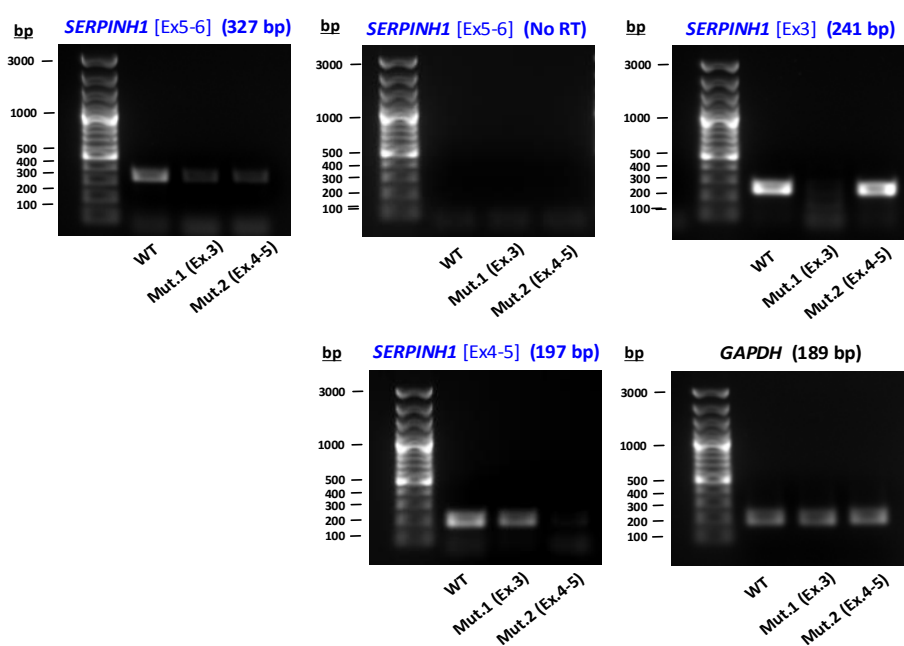

C

1

2 3

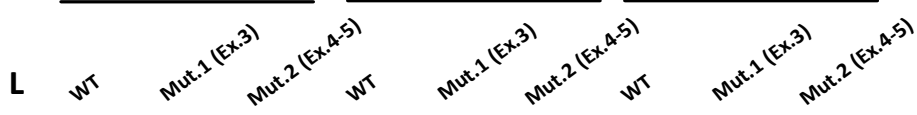

HSP47 (47 kDa)

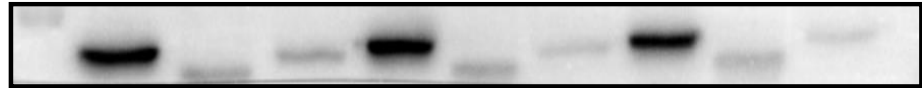

GAPDH (37 kDa)

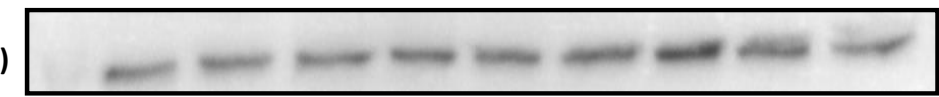

D
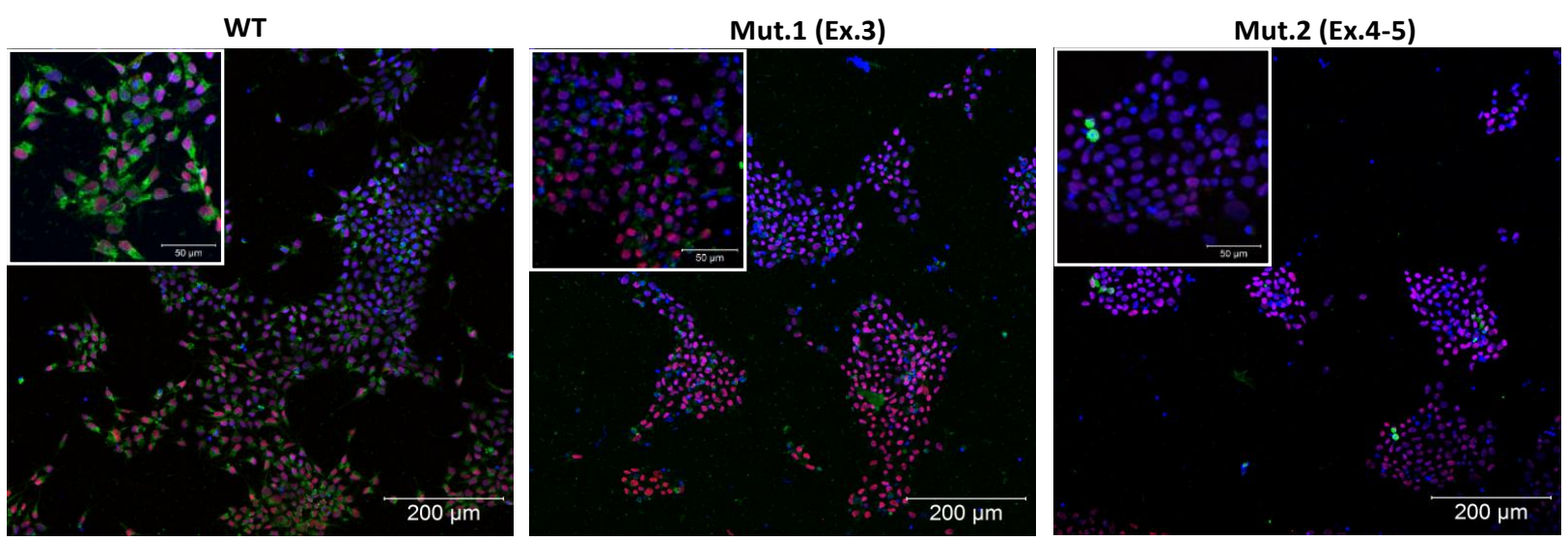

HSP47, OCT4, Hoechst

Figure 35. Confirmation of the HSP47 mutations in hES2 cells. (A) Schematic overview of human SERPINH1 mRNA (WT) and where the mutations (red) were introduced in Mut.1 (exon 3 mutation), Mut.2 (exon 4-5 mutation), and where primers were specifically targeted (blue) to validate respective target-specific deleted/mutated regions. Primers targeted; Exon 5-6 (localized out of the site-specific mutated regions), exon 3 and exon 4-5 (targeting the specific sites of mutation). (B) RT-PCR confirmed the site-specific mutations in Mut.1 (exon 3) and Mut.2 (exon 4-5) at RNA level. The 'no RT' (reverse transcriptase) controls indicated cDNA synthesis was performed in the absence of reverse transcriptase. Base pairs (bp). (C) Immunoblotting analysis demonstrates HSP47 protein expression was markedly reduced in both Mut.1 and Mut.2. Both mutations show band shifts that are the result of the mutated protein (3 biological replicates/group). (D) Immunofluorescence analysis confirmed the dramatic reduction of HSP47 (green) in Mut. 1 and Mut.2 pluripotent (red) hES2 cells compared to WT cells. 
Interestingly, Mut.1 shows a band shift that is a result of the mutated protein, which has 39 amino acids (aa) (aa 53-91 of the HSP47 protein sequence) less than WT. This consequently produced a smaller product size. This band shift was similarly observed in Mut.2, which has 30 amino acids (aa 229-258 of the HSP47 protein sequence) less than WT. This shift was however much less pronounced. Furthermore, immunohistochemical analysis additionally confirmed that HSP47 (green) showed negligible expression in pluripotent Mut.1 and Mut.2 cell lines, in contrast to WT cells (red, blue) (Figure 35D). A very small population of HSP47 positive cells were present in Mut.2, however since WT cDNA was not found to be present in both Mut.1 and Mut.2 cells at PCR level (Figure 35B), we can thus exclude that there was contamination of WT cells present in both mutant cell lines.

For ongoing future experiments, the functional consequences of the human HSP47 $\mathrm{KO}$ mutations in cardiac tissue formation and maturation will be assessed by utilizing the human EHM model. 


\section{Discussions:}

The heart is a complex multicellular organ which undergoes many critical changes during development and under pathological conditions. It is clear that both the CM and non-myocyte components in the heart play essential roles in the processes involved in the development of cardiac hypertrophy, remodelling and heart failure (Takeda and Manabe, 2011). cFBs represent the predominant non-myocyte population in the adult mammalian heart (Frangogiannis, 2013; Souders et al., 2009; Camelliti et al., 2004a) and while studies of CMs in the heart have been extensive, cFBs have received less attention. Emerging evidence suggests that cFBs are one of the key components actively participating not only in response to pathological injury and stress, but also in normal cardiac function (Kakker and Lee, 2009). It has also been suggested that $\mathrm{cFBs}$ are able to interact with $\mathrm{CMs}$ in a paracrine manner, via the influence of ECM secretion and remodelling, and via direct cell-cell interactions, which influence cardiac development, normal physiology and pathophysiology (Kakker and Le, 2009). The mechanisms of how cFBs contribute to the formation of a cardio-instructive environment remain largely undefined.

We hypothesized that the mechanical and paracrine effects of cFBs and its secreted ECM influence cardiac tissue formation and CM maturation. We were able to demonstrate that:

- $\quad$ FBs are essential for mouse and human engineered heart muscle formation.

- Integrin mediated FB-ECM interactions are essential for engineered heart muscle formation.

- FBs secrete a cardio-instructive ECM environment important in mediating heart muscle maturation.

- Collagen processing HSP47 is an important regulator of heart muscle formation and maturation. 


\subsection{Fibroblasts are essential for engineered heart muscle formation}

Engineered myocardium provides a simplified 3D cellular environment model to study cell-cell interactions. We utilized the in vitro EHM model of heart muscle development to investigate the role of $\mathrm{cFBs}$ in cardiac tissue development and maturation in mouse and human systems. Firstly, by use of genetically engineered ESC lines, we were able to successfully generate by directed differentiation highly purified CMs for both mouse (Figure 12) and human (Figure 13), to exclusively define the cellular constituents (CMs to non-myocytes) in the EHM model. Most technologies to generate engineered myocardium up until now have been mainly based on mixed or relatively non-purified cell populations enriched for CMs, derived from mouse ESCs (Guo et al., 2006), human ESCs (Tulloch et al., 2011) and rodent primary heart cells (Naito et al., 2006).

We were able to demonstrate that enriched mouse CMs alone mixed with collagen type I (the most abundant ECM protein in the myocardium) is insufficient to form compact functional cardiac tissue (Figure 14). Similar observations were found in the human collagen-based EHM (Tiburcy et al., unpublished data) and fibrin-based engineered myocardium (Zhang et al., 2013). Furthermore, studies from our lab have also revealed in mice that the addition of FB-conditioned medium, as well as the supplementation with additional growth factors and ECM proteins in matrigel to highly purified CMs and collagen type I is insufficient to rescue this failed tissue phenotype (Figure S1, Appendix A1, Christalla et al., unpublished data). These observations argue against the critical importance of paracrine mechanisms in exclusively mediating initial heart muscle formation.

We next tested whether the addition of cFBs would support EHM formation and tissue maturation. We indeed found that the addition of $25 \% \mathrm{cFBs}$ to EHMs resulted in the generation of mature compact tissue engineered myocardium with organotypic morphology and functionality in both mouse (Figure 14; Figure S1, Appendix A1, Christalla et al., unpublished data) and human (Tiburcy et al., unpublished data). These observations are in line with a previous study in our lab using a similar celldefined ( $25 \%$ cFBs mixed with $75 \%$ genetically purified mouse parthenogenetic stem cell-derived CMs) approach to generate EHMs, with the addition of matrigel (Didié et 
al., 2013). Our observations furthermore support a previous study from our lab which have shown that EHMs derived from mixed populations of rodent neonatal heart cells (containing a higher fraction of $\mathrm{cFBs}$ ) on collagen type I and Matrigel compared to constructs comprised of purer CM populations display improved contractile force generation and increased inotropic response (Naito et al., 2006).

Several other cardiac tissue engineering-based approaches have furthermore suggested that cFBs greatly enhance the growth and formation of well structured, functional myocardial tissue (Caspi et al., 2007; Nichol et al., 2008; Radisic et al., 2008) in addition to promoting CM survival and coupling (Kensah et al., 2012; Liau et al., 2011; Matsuura et al., 2011; Pfannkuche et al., 2010). Many of these studies above, including other reports in the field of cardiac tissue engineering have relied on the use of matrigel for the generation of cardiac tissue constructs. Given the fact that our study furthermore demonstrates the addition of ECM proteins and growth factor from matrigel are not critical for the generation of compact functional EHM in both mouse and human systems, this further supports the notion that cFBs are exclusively essential for the generation and support of myocardial tissue.

Several other findings have highlighted the importance of cFBs in playing a role in myocardial compaction during cardiac development. The embryonic pool of cFBs during development has been suggested to partially originate from the epicardium by epithelial-mesenchymal transformation (EMT) (Lie-Venema et al., 2007; Gittenberger-de Groot et al., 1998; Dettman et al., 1998; Cai et al., 2008) and several studies have shown these epicardial-derived cells (EPDCs) migrate into the ventricular wall of the embryonic heart, leading to myocardial compaction and maturation (Sengbusch et al., 2002; Pérez-Pomares et al., 2002; Ieda et al., 2009). Preventing the migration and invasion of EPDCs into the ventricular wall of the embryonic heart has been shown to result in myocardial non-compaction, causing lethal cardiac failure (Sengbusch et al., 2002; Pérez-Pomares et al., 2002). Our data suggests that cFBs exhibit a similar function by compaction of EHM. To further define the cellular mechanism underlying this observation, we investigated whether direct cell-cell interactions, paracrine signalling or other factors from cFBs mediate these beneficial effects. 


\subsection{Integrin mediated fibroblast-ECM interactions are essential for engineered heart muscle formation}

Integrin transmembrane receptors are fundamental components in the myocardium which function as mechanotransducers in mediating the interaction between the ECM and CMs or cFBs, in the process of normal cardiac development (Ross and Borg, 2001) and in response to physiological and pathological signals (Simpson et al., 1999; Parker and Ingber, 2007). Given the fact that CMs alone failed to develop functional and mature compact cardiac tissue in both mouse and human EHM, we thus investigated whether FB-specific mechanisms of mechanotransduction are responsible for the initial formation of EHMs.

We analysed the expression of collagen binding integrin chains; Itg $\alpha 1$, Itg $\alpha 2$ and Itg $\beta 1$ in purified CMs and FB single cell populations in monolayer culture, at both protein and RNA level (Figure 15, 16 and 17). As it has not been clearly demonstrated whether different types of FBs (besides cardiac origin) can improve tissue quality, the properties of FBs from both embryonic and cardiac origin were thus investigated in this study. Interestingly, we identified that FBs express higher levels of ITGA1, ITGA2 and ITGB1 in contrast to CMs in both mouse and human models at the protein and RNA level. In both mouse and human systems, we found Itg $\alpha 2$ transcribed in FBs, however negligible expression was detected in CMs. This is in agreement with previous findings which have demonstrated that Itga 2 is uniquely expressed in rodent cFBs but not by CMs (Terracio et al., 1991; Terracio et al., 1989). We confirmed a similar expression profile in human cells.

Although our data reveals that Itg $\beta 1$ expression is lower at both protein and RNA level in CMs compared to FBs in both mouse and human systems, $\operatorname{Itg} \beta 1$ has however been reported to be expressed in enriched mouse embryonic CMs (Ieda et al., 2009). The expression levels of these integrins may differ in a $3 \mathrm{D}$ environment or upon interaction with the ECM (Harston and Kuppuswamy, 2011), as it is becoming increasingly appreciated that cell receptor, protein and gene expression can differ between 2D and 3D culture environments (Edmondson et al., 2014; Pontes Soares et al., 2012). Further studies would be required to investigate whether integrin expression in FBs and CMs is altered upon interaction with the ECM. 
We next tested whether inhibition of integrin-mediated signalling functionally impairs initial EHM formation and tissue compaction, using collagen binding integrin chain blocking antibodies. We indeed found that blocking of $\operatorname{Itg} \beta 1$ (CD29) inhibits EHM formation in both mouse and human models (Figure 18). Since $\operatorname{Itg} \beta 1$ associates with both Itg $\alpha 1$ and Itg $\alpha 2$ subunits, and interacts with collagens, fibronectin and laminins (Barczyk et al., 2010), it is no surprise that blocking of Itg $\beta 1$ exhibits the most pronounced inhibitory effects on EHM formation. Furthermore, blocking of Itg $\alpha 2$ (CD49b) interactions has shown to inhibit initial EHM formation in the mouse (to a lesser extent than Itg $\beta 1$ blocking) and partially in the human model. Blocking of Itg $\alpha 1$ (CD49a) interactions however did not demonstrate to inhibit initial EHM formation. This is in agreement with several other studies which have demonstrated the role of $\operatorname{Itg} \beta 1$, in particular the $\alpha 2 \beta 1$ receptor (and to a lesser extent the $\alpha 1 \beta 1$ receptor) in mediating collagen gel contraction by FBs (Gullberg et al., 1990; Carver et al., 1995; Langholz et al., 1995; Schiro et al., 1991; Grinnell, 1994).

Other studies have highlighted the importance of integrin mediated signalling in the myocardium. Cardiac cells from chimeric mice constructed with a $\operatorname{Itg} \beta 1$ integrin-null allele exhibited delayed development and differentiation of cardiac lineage, as well as abnormal myofibrillogenesis (Fässler et al., 1996). A lack of $\operatorname{Itg} \beta 1$ expression in homozygous mice leads to embryonic lethality (Fässler and Meyer, 1995). Moreover, using Itg $\beta 1$ deficient embryonic stem cells for the in vitro differentiation of CMs, severely disrupted cardiomyogenesis and defective myofibrillogenesis was observed (Fässler et al., 1996). Furthermore, when Itg $\beta 1$ is inactivated exclusively in ventricular CMs, this resulted in myocardial fibrosis and heart failure (Shai et al., 2002). In addition, the disruption of $\operatorname{Itg} \beta 1$ function in CMs by a high level of dominant-negative Itg $\beta 1$ mutant under the control of the CM-specific $\alpha$-myosin heavy chain $(\alpha \mathrm{MHC})$ promoter, displayed a more severe pathological phenotype. These transgenic mice died perinatally and displayed fibrotic replacement of the myocardium (Keller et al., 2001). cFBs have also been suggested to play critical roles in cardiac remodelling via ECM-integrin interactions (Manso et al., 2009). These studies thus indicate that cell-ECM interactions via integrin signalling are essential for the organization and maintenance of the myocardium during development. 
We collectively show that FB populations express higher levels Itg $\beta 1$ compared to $\mathrm{CMs}$, and CMs alone failed to support the formation of a functional syncytium and organotypic maturation. We can thus conclude that ECM-Cell interactions mediated via integrin mechanotransduction signals in FBs are essential in mediating cardiac tissue formation.

\subsection{Fibroblasts secrete a cardio-instructive ECM environment important in mediating heart muscle maturation}

The ECM in the heart has been primarily recognized as a structural scaffold for embedding and supporting CMs and non-myocytes, in order to maintain functional integrity of the myocardium. It is becoming increasingly understood that the different ECM components within the heart provide a more complex insight into the biological and regulatory function of the cardiac ECM. We investigated the influence of the cFB-producing ECM environment in promoting cardiac organotypic maturation in our mouse EHM model (Figure 19; Christalla et al., unpublished data). Firstly, we identified that several ECM-related transcripts (Collal and Colla2), in addition to collagen-modifying transcripts, are massively upregulated during tissue cardiac maturation in cFB-supplemented EHMs (Figure 19A). Besides collagen type I, other gene transcripts which are mainly involved in collagen I fibrillogenesis and maturation were also highly expressed, such as Dcn and Bgn (Campbell et al., 2008), indicating that collagen type I modification is also important for EHM maturation. Furthermore, proteomic analysis revealed a number of ECM-related proteins, several collagen type I modifying proteins are exclusively expressed in mouse cFBsupplemented EHMs, compared to EHMs comprised only of enriched CMs (Figure 19B; Christalla et al., unpublished data). Proteins which are involved in collagen type I de novo synthesis, modification and organisation such as; PCOC1, PLOD2, PLOD3, P4HA1, P4HA2 and HSP47 (Goldsmith et al., 2013; Uzawa et al., 1999; Canty et al., 2005) indicate that cFBs are able to reorganize the collagen matrix and build a structured ECM environment. Furthermore, the high abundance of COL1A1 protein in EHMs containing purely CMs further point to the lack of tissue organization in the absence of cFBs. 
In line with the observations from our EHM model, it has been previously demonstrated that during cardiac development, embryonic cFBs development coincides with ventricular compaction, ECM production (including collagens and fibronectin) and growth factor secretion, which stimulates the proliferation of immature CMs through Itg $\beta 1$ signalling (Ieda, et al., 2009; Hornberger et al., 2000). Furthermore, the influence of mechanical stimulation on cultured cFBs has been shown to impact ECM gene expression (MacKenna, 2000). In addition to our observations in the mouse system, we identified that human FB populations (from both dermal and cardiac origin) highly express these similar ECM-related gene transcripts, in contrast to enriched human CMs (Figure 20). Interestingly we also identified that several laminin subtype transcripts: LAMA1, LAMA2 and LAMC3 were however exclusively highly abundant in human CMs, in contrast to FB populations. Whether or not these ECM-related genes are upregulated or down regulated in a $3 \mathrm{D}$ environment in human cardiac tissue formation and maturation however requires further investigation.

The binding of ECM to specific integrins in CMs and FBs has the potential to activate signalling cascades with a potential role in cell fate regulation. Several studies have highlighted the importance of the ECM environment in supporting CM differentiation of ESCs, derived from mouse (Haguchi et al., 2013; Baharvand et al., 2005) and human (Oberwallner et al., 2015). Additionally, an earlier study suggested that collagen I and laminins provide adequate cues for promoting the upregulation of $\mathrm{CM}$ maturation marker genes in rodents (LaNasa, 2009). Furthermore, the FB-derived microenvironment changes during development to promote stage-specific processes of CM differentiation, integration and maturation (Ieda et al., 2009). In rodents, collagen synthesis (Mays et al., 1991) and fibronectin expression (Farhadian et al., 1995) changes with development and integrin isoforms change simultaneously with the transition from CM proliferation to terminal differentiation (Maitra et al., 2000). Several studies have suggested the ECM from young developmental age promotes the expansion of CMs in vitro compared to older aged ECM (Ieda et al., 2009; Williams et al., 2014). Further studies to investigate the ECM environment constructed from FBs from different stages of development (and disease) in a defined 3D EHM model may provide further information of key proteins which can promote or impair formation of a functional myocardial syncytium. 
The complexity of the heterogeneous ECM environment produced from FBs provides spatial information for the cellular components and transmits mechanical forces that allow tissues to both develop and function efficiently in vivo (Goldsmith et al., 2013; Nelson and Tien, 2006). In conclusion, our findings collectively demonstrate that FBs mechanically support tissue formation and construct a heterogeneous ECM environment composed of primarily of collagens and collagen modifying proteins, to biologically construct a functional myocardial syncytium.

\subsection{Collagen processing HSP47 is an important regulator of heart muscle formation and maturation}

\subsubsection{HSP47 is highly expressed in fibroblasts}

Myocardial collagens provide both mechanical strength and structural support to the heart. In the healthy myocardium, fibrillar collagens play a critical role in guiding CM orientation, size and function (Porter and Turner, 2009) in order to facilitate efficient contraction, force transduction and electrical transmission of cells in the myocardium. It has not been demonstrated how alterations in ECM collagen modification and processing in cFBs can influence $\mathrm{CM}$ structure and function in the myocardium. Mutations of various types of collagen genes have also been identified to cause various collagen-related diseases including; Osteogenesis Imperfecta (OI), EhlersDanlos syndrome and Alport syndrome (Nagai et al., 2001). The collagen-specific molecular chaperone HSP47 is essential for the molecular maturation, processing and secretion of Collagens type I and IV and it has been shown that the disruption of the Hsp47 gene in mice is embryonically lethal (Nagai et al., 2001). No differences in the molecular forms of other predominant ECM proteins, including: collagen III, Laminin and Fibronectin were however detected in HSP47 KO mice (Nagai et al., 2001).

We firstly compared the expression of collagen processing HSP47 in FBs and CMs to demonstrate that HSP47 expression is highly abundant in both mouse and human FBs (Figure 21 and 22). Comparing the FB populations in human cells, we identified hFFBs express higher HSP47 protein levels compared to cFBs. On the contrary, cFBs possess higher SERPINHI transcript levels compared to hFFBs. Further studies will 
be required to compare regulatory mechanisms between both populations. Human ESC derived-CMs express both low levels of SERPINH1 and collagen type I transcripts (COL1A1 and COL1A2), accordingly only minimal procollagen I protein was detected in CMs compared to FBs (Figure 15D). We have also identified that HSP47 expression is highly abundant exclusively in mouse cFB-supplemented EHMs (Figure 19B). In agreement with previous observations, the expression of HSP47 under normal conditions is closely linked with the expression of collagens in various cell types and tissues (Nakai et al., 1990; Hirayoshi et al., 1991; Sauk et al., 1994; Kambe et al., 1994) and HSP47 expression is not detected in cells which do not synthesize collagen (Nagata and Hosokawa, 1996).

\subsubsection{Absence of HSP47 in mouse fibroblasts impairs ECM Collagen type I secretion and fibrillar organisation}

Based on the observation that HSP47 is predominantly expressed in collagenproducing FBs, we next set out to investigate its influence on mouse CM maturation by utilizing HSP47 KO MEFs derived from HSP47KO mice (Nagai et al., 2001). We confirmed the absence of HSP47 in MEFs at both mRNA and protein levels (Figure 3.12), as previously shown (Nagai et al., 2001; Ishida et al., 2006). Our study revealed that HSP47 partially colocalizes in the same compartment with PDI and procollagen 1 in WT MEFs, however the expression pattern of procollagen 1 in HSP47 KO cells reflects that it is accumulated in the intracellular ER compartment (Figure 24). Additionally, we found that PDI protein levels were partially elevated in HSP47 KO MEFs (Figure 25). PDI is another ER resident chaperone protein (Wang et al., 1998; Gilbert, 1997) that also has functions during procollagen synthesis specifically in the formation of procollagen inter-chain disulphide bonds within the ER (Koivu et al., 1987; Gelse et al., 2003; Canty et al., 2005). Our observations are in close agreement with other studies, which have demonstrated that HSP47 transiently associates with procollagen in the ER and dissociates before reaching the cis-Golgi (Satoh et al., 1996). Further immunoflourescence experiments to assess whether HSP47 partially colocalizes with GM130 (a marker of the cis-Golgi compartment) would be needed to specify the localization within the ER and Golgi compartments. 
It has also been reported that misfolded procollagen 1 accumulates within the ER in HSP47 KO MEFs, resulting in ER dilation (Ishida et al., 2006). This partial increase in PDI levels we observed may thus be a compensatory effect to protect these cells from the accumulation of procollagen we observed in the ER in HSP47 KO MEFs. In contrast, a previous study however has shown that procollagen misfolded aggregates are eliminated from the ER via the autophagic lysosome pathway, as a protection strategy in HSP47KO MEFs (Ishida et al., 2009). Further experiments to examine the expression of ER markers: IRE1 $\alpha$, PERK, ATF6 and CHOP1, via immunoblotting and qPCR (Oslowski and Urano, 2011) would be needed to provide further information to whether the accumulation of misfolded procollagen in HSP47 KO MEFs result in ER stress.

We were able to demonstrate that HSP47 KO MEFs display markedly reduced secretion of soluble collagens after $72 \mathrm{hrs}$ (Figure 26). Additional time points could be assessed in the future to determine differences in the rate of collagen secretion. Previous findings however have reported that the secretion of collagen type I and IV is distinctly delayed in HSP47 KO MEFs (Ishida et al., 2006; Marsuoka et al., 2004), probably due to improper formation of the procollagen molecule structure. Importantly, Ishida et al., reported that $\mathrm{P} 4 \mathrm{H}$ is also clearly detected in $\mathrm{HSP} 47 \mathrm{KO}$ MEFs, indicating that improper collagen triple helix formation in these cells is not due to insufficient prolyl-hydroxylation (Ishida et al., 2006).

As we found that collagen defective HSP47 KO MEFs exhibit reduced collagen secretion after $72 \mathrm{hrs}$, we also demonstrated that the incorporation of collagen type I chains; COL1A1 and COL1A2 into the ECM is dramatically reduced in HSP47 KO MEFs (Figure 27B). We were able to confirm these findings via proteomic analysis (Figure 28). Interestingly, and despite an evident reduction in collagen secretion and collagen type I ECM incorporation in HSP47 KO MEFs, we observed that there is a trend towards a higher abundance of Collal and Colla2 chain transcripts at mRNA level in these cells compared to WT (Figure 27C). Nagai et al., reported that there were no significant differences in Collal and Colla2 mRNA levels between WT and HSP47 KO embryos (Nagai et al., 2001), however a more recent study from this group conditionally inactivated the $H s p 47$ gene in chondrocytes using a Cre-LoxP system in mice and revealed that Collal mRNA is upregulated in mouse embryos 
(Masago et al., 2012). In relation to our observations, HSP47 ablation in MEFs might thus cause the compensatory production of procollagen, although collagen secretion is impaired. This may also account for why we observed higher PDI protein expression in HSP47 KO MEFs.

Taken together, our data suggests that there is defective collagen type I processing and secretion in the absence of HSP47. Interestingly, proteomic analysis also revealed that Periostin (POSTN) expression is absent in HSP47 KO secreted ECMs. POSTN has been demonstrated to promote the proliferation and myofibroblast differentiation of FBs (Crawford et al., 2015). Further experiments would need to be performed to determine whether HSP47 KO MEFs are able differentiate into a myofibroblast phenotye in culture. POSTN has furthermore been implicated in the regulation of collagen type I fibrillogenesis and organization (Norris et al., 2007). Collagen fibrils from POSTN-deficient mice have shown to be reduced in size, disorganized and less efficiently cross-linked (Snider et al., 2009; Norris et al., 2007; Shimazaki et al., 2008). Furthermore, POSTN is expressed during fetal cardiac development (Bowers et al., 2010) and is found to promote CM proliferation in vitro and improved heart function after myocardial infarction in adult rodents (Kühn et al., 2007). Interestingly, we also identified that several extracellular vesicular exosome proteins were exclusively incorporated into the HSP47 KO ECM (Figure 28). It has been recently demonstrated that paracrine intercellular communication in between FBs and CMs occurs via exosomes and microvesicles (Bang et al., 2015). Furthermore, it has been suggested that exosomes may also have a functional role in selectively secreting harmful/damaged proteins, in order to alleviate intracellular stress conditions and preserve intracellular protein homeostasis (Baixauli et al., 2014). This may suggest that protein trafficking and secretion may be impaired in HSP47 KO cells. Since the mass spectrometry experiments were performed on 1 sample/group with 2 technical replicates, additional repeats of this experiment would be required to confirm these findings and further investigate whether a feedback loop regulation exists between these proteins and the mechanisms behind it.

We next examined that structure of the ECM deposited from collagen defective HSP47 KO MEFs and revealed that this ECM showed dramatically reduced fibrillar structures, which appeared thinner with more frequent branchlike structures (Figure 
30). Collagen type I deposition is markedly reduced and displayed thinner, reduced fibrous ECM networks. The deficient POSTN expression we observe in HSP47 KO ECM may further account for why we observed disrupted collagen type I fibrils, however further studies at both protein and RNA level in HSP47 KO MEFs would be required to verify this observation.

These findings are in line with previous studies by Nagata and colleagues, which have extensively shown that: (1) HSP47 KO MEFs produce abnormally thin and frequently branched collagen type I fibrils evidenced by electron microscopy (Ishida et al., 2006), a phenotype that could be restored by transient expression of HSP47 in HSP47 KO cells (Ishida et al., 2006); (2) Collagens secreted from HSP47 KO MEFs were more susceptible to protease digestion, suggesting that there is defective triple helix formation of collagens I and IV in the ER of these cells (Nagai et al., 2001; Matsuoka et al., 2004); (3) Immature collagen type I with unprocessed $\mathrm{N}$-terminal propeptides are secreted from HSP47 KO MEFs and accumulated in the ECM (Ishida et al., 2006). Taken together, our observations corroborated by these studies demonstrate that HSP47 is required for correct folding of procollagen in the ER, which is necessary for efficient secretion, processing and fibril formation of collagen type I.

\subsubsection{Defective ECM Collagen type I secretion and organisation impairs cardiomyocyte homeostasis and maturation}

As fibrillar collagen plays a critical role in maintaining cardiac shape, size and function (Porter and Turner, 2009), we next investigated how defective collagen processing HSP47 influences the cardio-instructive properties of the ECM. We firstly demonstrated that purified mESC-CMs cultured on WT MEF-derived ECM displayed a mature CM phenotype with advanced sarcomeric organization and morphological features with regular cross striations and primarily rod shaped bodies, which are orientated along the collagen type I microfibrillar structure (Figure 31). Interestingly, CMs exhibited an impaired phenotype when cultured on collagen defective HSP47 KO MEF-derived ECMs, displayed by a rounded, flat, immature morphology with disorganized sarcomeric structures. 
The structure and orientation of ECM fibrillar collagen has shown to be important in determining CM structure. Mechanically aligned collagen fibrils have the capacity to elongate muscle cells along its parrellel axis (Vandenburgh and Karlisch, 1989). Furthermore, in vitro studies have demonstrated that microcontact printing parallel lines of ECM proteins on 2D substrates induce CM alignment (Pong et al., 2011; Feinberg et al., 2012; Grosberg et al., 2011). Alignment of CMs is important for both electromechanical coupling and contractile force generation (Black et al., 2009; Chung et al., 2007) and it can also promote differentiation and maturation of cardiac progenitor cells (Domian et al., 2009). Further immunohistochemical stainings for cardiac gap junction protein $\mathrm{Cx} 43$ and $\mathrm{Cx} 45$ would provide further information into possible differences in electrical coupling of CMs on collagen-defective ECMs.

Due to FBs depositing dense ECM networks of insoluble proteins (after 11 days) in culture, we tested several different published protocols in attempt to solubilise and quantity of ECM protein deposited on plates (Beacham et al., 2007; DeQuach et al., 2010; Harvey et al., 2013 Turoverova et al., 2009). None were able to aid in successful homogeneous solubilisation of FB-derived ECMs. We are thus unable to clearly distinguish whether this impaired CM phenotype is a consequence of the immature unprocessed collagen depostion, or due to the insufficient amount of total collagen deposited from HSP47 KO MEFs. Future experiments using higher concentrations of SDS $(\geq 3 \%)$ may aid in successful solubilization of ECM. In addition, the stiffness of the myocardial ECM enviroment may also be important for CM maturation and function (Majkut et al., 2013; Young and Engler, 2011). Additional studies to assess the biophyisal properties (stiffness) of these ECMs produced from these MEFs would provide us further information of how defective collagen processing in FBs can influence the mechanical enviroment of the ECM, and $\mathrm{CM}$ structure and function. From these obervations, we can however conclude that defective collagen processing and secretion from HSP47 KO MEFs impairs the ECM cardio-instructive properties in guiding immature CMs towards a mature phenotype.

We next investigated the influence of defective ECM collagen type I in cardiac tissue formation and maturation utilizing our 3D EHM model of heart muscle development. We found that HSP47 KO MEF-supplemented EHMs demonstrate a lower degree of 
maturation (Figure 32), which is characterized by the failure to form compact mature functional tissue. These tissues exhibited an impaired $\mathrm{CM}$ phenotype, with disorganized sarcomeric structures, immature rounded morphology and failed to form tightly pack, dense, aligned CM muscle bundles. Furthermore, these EHMs failed to develop contractile force which is representative of a impaired functional syncytium. This obervation may likely be due to reduced Itgbl expression and impairment of mechanical signalling in HSP47 KO MEFs (Figure 33), which may explain for why these tissues failed to condense. We did not observe a significant difference in ITGB1 at protein level ( $n=3$ group), however further experiments with more replicates/group may elimate variability and demonstrate signficant differences. The defective ECM collagen type I derived from HSP47KO MEFs (observed in 2D culture) may furthermore impair CM structure and organisation within the tissue. It would also be critical to assess whether ER stress is induced within these tissues, by assessing markers of ER stress at both protein and mRNA levels. We could speculate that this reduction in Itgbl transcripts could be due to defective protein trafficking and ER stress induction in HSP47 KO MEFs. This however requires further investigation. In addition, further experiments to determine whether this impaired EHM phenotype could be rescued by transiently expressing HSP47 into HSP47 KO cells via transfection with a mouse HSP47 expression vector (Ishida et al., 2006; Nagai et al., 2000), would provide additional evidence that HSP47 in FBs is critical for EHM formation and maturation.

Although the role of HSP47 in the myocardium has currently not been extensively studied, the influence of HSP47 inhibition in cardiac remodelling after myocardial infarction has been previously investigated (Hagiwara et al., 2011). This study demonstrated that HSP47 antisense-oligonucleotides could reduce ligated left anterior descending (LAD) coronary artery-induced myocardial infarction in rats. Rats with ligated LAD coronary artery exhibited an upregulation of HSP47 expression, remodelling of the left ventricle, and cardiac dysfunction. In contrast, rats with ligated LAD coronary artery treated with antisense oligonucleotides had significantly reduced HSP47 expression, cardiac remodelling, and improved cardiac function (Hagiwara et al., 2011). HSP47 has been closely related to the development of many different types of fibrosis (Kakugawa et al., 2010; Masuda et al., 1994; Hagiwara et al., 2007; Kakugawa et al., 2004). Several studies have also suggested that the pro-fibrotic 
cytokine TGF- $\beta$ can induce HSP47 expression (Sasaki et al., 2002). In addition, the microRNA-29 (miR-29) family act as regulators in cardiac fibrosis (van Rooij et al., 2008) and have been recently suggested to target and down regulate HSP47 expression (Zhang et al., 2014; Zhu et al., 2015; Zhao et al., 2014; Yamamoto et al., 2013)

Another more recent study has demonstrated that administration of the statin Atorvastatin, ameliorated cardiac fibrosis and improved left ventricular diastolic function in a rat model of hypertensive diastolic heart failure while reducing the expression of HSP47 in fibrotic hearts and cFBs (Akahori et al., 2014). In addition to mouse models, previous studies have revealed that the SERPINHI is one of the genes responsible for recessive OI in both human (Christiansen et al., 2010) and dog (Lindert et al., 2015; Drogemuller et al., 2009). It has been recently reported that patients with IO have increased risk of heart disease and in particular valve diseases, which include dilation of the aorta, compared to healthy control patients (Ashournia et al., 2015; Karamifar et al., 2013; Rudunovic et al., 2011). Taken together, these data along with our findings demonstrate the importance of collagen processing HSP47 as a key regulator of ECM homeostasis, which can thus impact cardiac remodelling and function.

\subsubsection{Generation and validation of human HSP47 mutant cell lines}

From our observations in the mouse model, we wanted to further investigate whether (1) functional mutations in the human HSP47 protein impairs collagen type I processing and secretion in human FBs, and whether, (2) alterations in ECM collagen influence CM structure and tissue functionality, by modelling this in vitro in human 3D EHMs. Taking exclusive observations of genetic mutations in the Hsp47/SERPINH1 gene in mice (Nagai et al., 2001) and in human (Christiansen et al., 2010), we utilized the CRISPR/Cas9 system to mutate site specific regions of the SERPINH1 gene in pluropotent hES2 cells. We were able to successfully generate 2 in-frame mutation cell lines: Mut.1 and Mut.2. The Mut.1 cell line has a 117 bp deletion exclusively in exon 3 of SERPINH1, confirms the missense mutation 
(c.233T >C, p.Leu78Pro) in human OI patients with an autosomal-recessive disease. (Christiansen et al., 2010). The Mut.2 cell line has a 200 bp deletion in exon 4-5 (end of exon 4 and start of exon 5 [90 bp of the coding DNA sequence], and the intron in between $[110 \mathrm{bp}]$ ), covering a similar deleted region in HSP47 KO mouse model (Nagai et al., 2001).

We confirmed the presense of the mutations in Mut.1 (Exon 3) and Mut.2 (Exon 4-5) in the SERPINH1 gene (Figure 35B). HSP47 protein levels were dramatically reduced in both mutant lines compared to WT. The smaller protein size observed in Mut.1 samples (Figure 35C) is the consequence of a mutated protein sequence deletion (39 aa). The reported human OI-study revealed that exon 3 mutations in SERPINHI generated an unstable protein which is degraded, but could still be detected in dermal FBs (Christiansen, et al., 2010). This study also concluded that the overall rate of procollagen type I production is effectively normal, but triple helical conformation of secreted collagen type I (failure to mature) is altered (Christiansen, et al., 2010). They were however not able to conclude whether the small remaining detected protein is still functional. They speculated that if this protein remained functional, this may account for the less severe phenotype and differences in effects observed compared to the more severe phenotype in the HSP47 KO mouse model (Nagai et al., 2001; Ishida et al., 2006). These observations may explain for why there were small detected amounts of protein expressed in Mut.1.

Immunostaining analysis of Mut.2 cells revealed a negligible number of single cells showed positive staining (Figure 35D). Since RT-PCR confirmed the presence of the mutation in both cell lines, we can confirm from these findings that no contaminating WT cDNA is present in both homozygous mutations (Figure 35B). As WT cells appear to grow faster than mutant cells and that these positively expressed cells fail to outgrow the mutated cells in culture, we can furthermore exclude that no WT contaminants were present in the both mutant cell lines. In comparision to HSP47 KO mice that were generated via homologous recombination (see Figure 23), no HSP47 was detected at protein level in MEFs from HSP47 KO homozygous mice (Figure 24; Nagai et al., 2001; Ishida et al., 2006). Since our human site-specific mutation generated an inframe deletion, this may account for why we observe minor HSP47 protein expression in the Mut.2. We have no information on which epitope region in 
the HSP47 protein sequence the monoclonal HSP47 antibody used in all studies, recognises. Whether this detected protein is functional or not needs to be determined. In addition, a mutation in the C-terminal end of the HSP47 protein (c.977C>T, p.L326P) in OI-Dachshunds, has also been reported to impair collagen processing, secretion and collagen type I cross-linking (Lindert et al., 2015; Drogemuller et al., 2009). Mutant HSP47 protein is expressed as a stable protein, however its level is reduced to half the control level. This further demonstrates the critical importance of HSP47 as an ER chaperone involved in collagen type I processing, secretion and maturation.

The next critical experiments will be to test whether these mutations have functional effects, by differentiating these mutant hES2 lines, isolating the non-myocyte cellular fractions and characterize HSP47 expression, in addition to mesenchymal/FB markers, including: VIM, FSP-1, Procollagen 1, Platelet-derived growth factor (PDGF), in addition to surface markers: CD90, CD105 and CD73. Karyotyping would be necessary to rule out for any chromosomal abnormalities from these genetic mutations. Subsequent experiments would be necessary to examine whether these human HSP47 mutant non-myocytes have functional consequences in regards to collagen type I processing, secretion and ER stress. Critical experiments would finally need to be performed to assess whether these mutant cells functionally impair cardiac tissue formation in our human EHM model of heart muscle development.

This would thus provide us further evidence in human that: (1) the interaction between FB-secreted ECM collagen type I and CMs is important in controlling cardiac tissue homeostasis and maturation in the myocardium, and (2) HSP47 represents a attractive therapeutic target to specifically control collagen type I processing and maturation and support CMs in collagen-related diseases in the heart, such as cardiac fibrosis. 


\section{Summary and Perspectives}

In conclusion to these studies, we have collectively shown that cFBs are necessary for both the generation and maturation of a functional organotypic engineered myocardium, utilizing both mouse and human EHM models of heart muscle development. Firstly, we established that FB-ECM interactions mediated via integrins is essential in mediating cardiac tissue formation. Furthermore, FBs secrete a cardioinstructive ECM environment composed of primarily of collagens and collagen modifying proteins, important in mediating heart muscle maturation.

Moreover, we identified the collagen-specific molecular chaperone HSP47 in FBs to be an important regulator of cardiac tissue formation and maturation. Utilizing HSP47 KO MEFs, we demonstrated the absence of HSP47 impairs both the deposition of ECM-collagen and CM morphology and functionality in both recently established 2D and 3D EHM mouse culture systems. We identified these impairments in EHM formation and maturation may be due to: (1) reduced Itgbl expression and impairment of mechanical signalling in HSP47 KO MEFs; (2) defective ECM collagen type I deposition and organisation, which may impair CM structure and organisation. Additional experiments to determine whether this impaired EHM phenotype could be rescued by transient HSP47 expression would provide supporting evidence that HSP47 is critical for heart muscle formation and maturation.

Further critical experiments need to be performed to assess whether human HSP47 mutant stromal cells (generated via the CRISPR/Cas9 system) can also functionally impair cardiac tissue formation in the human EHM model. This would provide us additional evidence in the human system that the interaction between FB-secreted collagen type I and CMs is important in controlling cardiac tissue homeostasis and maturation. HSP47 may thus represent an attractive therapeutic target in regulating cardiac tissue homeostasis and support CMs in collagen-related diseases in the heart such as cardiac fibrosis. 


\section{Bibliography}

Abercrombie, M., and G. A. Dunn. 1975. Adhesions of fibroblasts to substratum during contact inhibition observed by interference reflection microscopy, Exp Cell Res, 92: 57-62.

Abraham, W. T., B. H. Greenberg, and C. W. Yancy. 2008. Pharmacologic therapies across the continuum of left ventricular dysfunction, Am J Cardiol, 102: 21G-28G.

Akahori, H., T. Tsujino, Y. Naito, M. Matsumoto, N. Sasaki, T. Iwasaku, A. Eguchi, H. Sawada, S. Hirotani, and T. Masuyama. 2014. Atorvastatin ameliorates cardiac fibrosis and improves left ventricular diastolic function in hypertensive diastolic heart failure model rats, J Hypertens, 32: 1534-41; discussion 41.

Akins, R. E., R. A. Boyce, M. L. Madonna, N. A. Schroedl, S. R. Gonda, T. A. McLaughlin, and C. R. Hartzell. 1999. Cardiac organogenesis in vitro: reestablishment of three-dimensional tissue architecture by dissociated neonatal rat ventricular cells, Tissue Eng, 5: 103-18.

Alam, N., H. L. Goel, M. J. Zarif, J. E. Butterfield, H. M. Perkins, B. G. Sansoucy, T. K. Sawyer, and L. R. Languino. 2007. The integrin-growth factor receptor duet, J Cell Physiol, 213: 649-53.

Alford, P. W., A. P. Nesmith, J. N. Seywerd, A. Grosberg, and K. K. Parker. 2011. Vascular smooth muscle contractility depends on cell shape, Integr Biol (Camb), 3: 1063-70.

Anders, S., and W. Huber. 2010. Differential expression analysis for sequence count data, Genome Biol, 11: R106.

Ashburner, M., C. A. Ball, J. A. Blake, D. Botstein, H. Butler, J. M. Cherry, A. P. Davis, K. Dolinski, S. S. Dwight, J. T. Eppig, M. A. Harris, D. P. Hill, L. IsselTarver, A. Kasarskis, S. Lewis, J. C. Matese, J. E. Richardson, M. Ringwald, G. M. Rubin, and G. Sherlock. 2000. Gene ontology: tool for the unification of biology. The Gene Ontology Consortium, Nat Genet, 25: 25-9.

Ashournia, H., F. T. Johansen, L. Folkestad, A. C. Diederichsen, and K. Brixen. 2015. Heart disease in patients with osteogenesis imperfecta - A systematic review, Int $J$ Cardiol, 196: 149-57.

Aszodi, A., K. R. Legate, I. Nakchbandi, and R. Fassler. 2006. What mouse mutants teach us about extracellular matrix function, Annu Rev Cell Dev Biol, 22: 591-621.

Atanassov, I., and H. Urlaub. 2013. Increased proteome coverage by combining PAGE and peptide isoelectric focusing: comparative study of gel-based separation approaches, Proteomics, 13: 2947-55.

Baharvand, H., M. Azarnia, K. Parivar, and S. K. Ashtiani. 2005. The effect of extracellular matrix on embryonic stem cell-derived cardiomyocytes, J Mol Cell 
Cardiol, 38: 495-503.

Baixauli, F., López-Otín, C., \& Mittelbrunn, M. 2014. Exosomes and Autophagy: Coordinated Mechanisms for the Maintenance of Cellular Fitness, Frontiers in Immunology, 5, 403.

Banerjee, I., J. W. Fuseler, R. L. Price, T. K. Borg, and T. A. Baudino. 2007. Determination of cell types and numbers during cardiac development in the neonatal and adult rat and mouse, Am J Physiol Heart Circ Physiol, 293: H1883-91.

Bang, C., Batkai, S., Dangwal, S., Gupta, S. K., Foinquinos, A., Holzmann, A., Just, A., Remke, J., Zimmer, K., Zeug, A., Ponimaskin, E., Schmiedl, A., Yin, X., Mayr, M., Halder, R., Fischer, A., Engelhardt, S., Wei, Y., Schober, A., Feidler, J., Thum, T. 2014. Cardiac fibroblast-derived microRNA passenger strand-enriched exosomes mediate cardiomyocyte hypertrophy, The Journal of Clinical Investigation, 124(5), 2136-2146.

Barczyk, Malgorzata, Sergio Carracedo, and Donald Gullberg. 2010. Integrins, Cell and Tissue Research, 339: 269-80.

Baudino, T. A., W. Carver, W. Giles, and T. K. Borg. 2006. Cardiac fibroblasts: friend or foe?, Am J Physiol Heart Circ Physiol, 291: H1015-26.

Beacham, D. A., M. D. Amatangelo, and E. Cukierman. 2007. Preparation of extracellular matrices produced by cultured and primary fibroblasts, Curr Protoc Cell Biol, Chapter 10: Unit 109.

Bell, E., B. Ivarsson, and C. Merrill. 1979. Production of a tissue-like structure by contraction of collagen lattices by human fibroblasts of different proliferative potential in vitro, Proc Natl Acad Sci U S A, 76: 1274-8.

Bellis, S. L. 2011. Advantages of RGD peptides for directing cell association with biomaterials, Biomaterials, 32: 4205-10.

Berk, B. C., K. Fujiwara, and S. Lehoux. 2007. ECM remodeling in hypertensive heart disease, J Clin Invest, 117: 568-75.

Berrier, A. L., and K. M. Yamada. 2007. Cell-matrix adhesion, J Cell Physiol, 213: 565-73.

Black, L. D., 3rd, J. D. Meyers, J. S. Weinbaum, Y. A. Shvelidze, and R. T. Tranquillo. 2009. Cell-induced alignment augments twitch force in fibrin gel-based engineered myocardium via gap junction modification, Tissue Eng Part A, 15: 3099108.

Boheler, K. R., L. Carrier, C. Chassagne, D. de la Bastie, J. J. Mercadier, and K. Schwartz. 1991. Regulation of myosin heavy chain and actin isogenes expression during cardiac growth, Mol Cell Biochem, 104: 101-7.

Bonaldo, P., P. Braghetta, M. Zanetti, S. Piccolo, D. Volpin, and G. M. Bressan. 1998. Collagen VI deficiency induces early onset myopathy in the mouse: an animal model for Bethlem myopathy, Hum Mol Genet, 7: 2135-40. 
Boudoulas, K. D., and A. K. Hatzopoulos. 2009. Cardiac repair and regeneration: the Rubiks cube of cell therapy for heart disease, Dis Model Mech, 2: 344-58.

Bowers, S. L., I. Banerjee, and T. A. Baudino. 2010. The extracellular matrix: at the center of it all, J Mol Cell Cardiol, 48: 474-82.

Bradford, Marion M. 1976. A rapid and sensitive method for the quantitation of microgram quantities of protein utilizing the principle of protein-dye binding, Analytical Biochemistry, 72: 248-54.

Bray, M. A., S. P. Sheehy, and K. K. Parker. 2008. Sarcomere alignment is regulated by myocyte shape, Cell Motil Cytoskeleton, 65: 641-51.

Bristow, M. R. 2000. beta-adrenergic receptor blockade in chronic heart failure, Circulation, 101: 558-69.

Brown, R. D., S. K. Ambler, M. D. Mitchell, and C. S. Long. 2005. The cardiac fibroblast: therapeutic target in myocardial remodeling and failure, Annu Rev Pharmacol Toxicol, 45: 657-87.

Brutsaert, D. L. 2003. Cardiac endothelial-myocardial signaling: its role in cardiac growth, contractile performance, and rhythmicity, Physiol Rev, 83: 59-115.

Burkin, D. J., and S. J. Kaufman. 1999. The alpha7beta1 integrin in muscle development and disease, Cell Tissue Res, 296: 183-90.

Burridge, K., and M. Chrzanowska-Wodnicka. 1996. Focal adhesions, contractility, and signaling, Annu Rev Cell Dev Biol, 12: 463-518.

Bursac, N., M. Papadaki, R. J. Cohen, F. J. Schoen, S. R. Eisenberg, R. Carrier, G. Vunjak-Novakovic, and L. E. Freed. 1999. Cardiac muscle tissue engineering: toward an in vitro model for electrophysiological studies, Am J Physiol, 277: H433-44.

Butt, R. P., G. J. Laurent, and J. E. Bishop. 1995. Collagen production and replication by cardiac fibroblasts is enhanced in response to diverse classes of growth factors, Eur J Cell Biol, 68: 330-5.

Cai, C. L., J. C. Martin, Y. Sun, L. Cui, L. Wang, K. Ouyang, L. Yang, L. Bu, X. Liang, X. Zhang, W. B. Stallcup, C. P. Denton, A. McCulloch, J. Chen, and S. M. Evans. 2008. A myocardial lineage derives from Tbx18 epicardial cells, Nature, 454: 104-8.

Calderone, A., S. Bel-Hadj, J. Drapeau, V. El-Helou, H. Gosselin, R. Clement, and L. Villeneuve. 2006. Scar myofibroblasts of the infarcted rat heart express natriuretic peptides, J Cell Physiol, 207: 165-73.

Calderwood, D.A. 2004. Integrin activation, J Cell Sci, 117:657-666.

Camelliti, P., G. P. Devlin, K. G. Matthews, P. Kohl, and C. R. Green. 2004a. Spatially and temporally distinct expression of fibroblast connexins after sheep ventricular infarction, Cardiovasc Res, 62: 415-25. 
Camelliti, P., C. R. Green, I. LeGrice, and P. Kohl. 2004b. Fibroblast network in rabbit sinoatrial node: structural and functional identification of homogeneous and heterogeneous cell coupling, Circ Res, 94: 828-35.

Campbell, P. H., D. L. Hunt, Y. Jones, F. Harwood, D. Amiel, J. H. Omens, and A. D. McCulloch. 2008. Effects of biglycan deficiency on myocardial infarct structure and mechanics, Mol Cell Biomech, 5: 27-35.

Canty, E. G., and K. E. Kadler. 2005. Procollagen trafficking, processing and fibrillogenesis, J Cell Sci, 118: 1341-53.

Carrier, R. L., M. Papadaki, M. Rupnick, F. J. Schoen, N. Bursac, R. Langer, L. E. Freed, and G. Vunjak-Novakovic. 1999. Cardiac tissue engineering: cell seeding, cultivation parameters, and tissue construct characterization, Biotechnol Bioeng, 64: $580-9$.

Carver, Wayne, Ivan Molano, Titus A. Reaves, Thomas K. Borg, and Louis Terracio. 1995. Role of the $\alpha 1 \beta 1$ integrin complex in collagen gel contraction in vitro by fibroblasts, Journal of Cellular Physiology, 165: 425-37.

Caspi, O., A. Lesman, Y. Basevitch, A. Gepstein, G. Arbel, I. H. Habib, L. Gepstein, and S. Levenberg. 2007. Tissue engineering of vascularized cardiac muscle from human embryonic stem cells, Circ Res, 100: 263-72.

Chacko, K. J. 1976. Observations on the ultrastructure of developing myocardium of rat embryos, J Morphol, 150: 681-709.

Chen, C. S., M. Mrksich, S. Huang, G. M. Whitesides, and D. E. Ingber. 1997. Geometric control of cell life and death, Science, 276: 1425-8.

Chen, C. S., J. Tan, and J. Tien. 2004. Mechanotransduction at cell-matrix and cellcell contacts, Annu Rev Biomed Eng, 6: 275-302.

Chen, W., and N. G. Frangogiannis. 2013. Fibroblasts in post-infarction inflammation and cardiac repair, Biochim Biophys Acta, 1833: 945-53.

Chien, K. R., I. J. Domian, and K. K. Parker. 2008. Cardiogenesis and the complex biology of regenerative cardiovascular medicine, Science, 322: 1494-7.

Chilton, L., W. R. Giles, and G. L. Smith. 2007. Evidence of intercellular coupling between co-cultured adult rabbit ventricular myocytes and myofibroblasts, $J$ Physiol, 583: 225-36.

Christalla, P., J. E. Hudson, and W. H. Zimmermann. 2012. The cardiogenic niche as a fundamental building block of engineered myocardium, Cells Tissues Organs, 195: 82-93.

Christiansen, H. E., U. Schwarze, S. M. Pyott, A. AlSwaid, M. Al Balwi, S. Alrasheed, M. G. Pepin, M. A. Weis, D. R. Eyre, and P. H. Byers. 2010. Homozygosity for a missense mutation in SERPINH1, which encodes the collagen chaperone protein HSP47, results in severe recessive osteogenesis imperfecta, Am J Hum Genet, 86: 389-98. 
Chung, C. Y., H. Bien, and E. Entcheva. 2007. The role of cardiac tissue alignment in modulating electrical function, J Cardiovasc Electrophysiol, 18: 1323-9.

Clarke, E. P., G. A. Cates, E. H. Ball, and B. D. Sanwal. 1991. A collagen-binding protein in the endoplasmic reticulum of myoblasts exhibits relationship with serine protease inhibitors, J Biol Chem, 266: 17230-5.

Conner, D. A. 2001. Mouse embryo fibroblast (MEF) feeder cell preparation, Curr Protoc Mol Biol, Chapter 23: Unit 232.

Crawford, Justin, Karen Nygard, Bing Siang Gan, and David Brian O'Gorman. 2015. Periostin induces fibroblast proliferation and myofibroblast persistence in hypertrophic scarring, Experimental Dermatology, 24: 120-26.

Curtis, M. W., and B. Russell. 2011. Micromechanical regulation in cardiac myocytes and fibroblasts: implications for tissue remodeling, Pflugers Arch, 462: 105-17.

de Jong, S., T. A. van Veen, J. M. de Bakker, and H. V. van Rijen. 2012. Monitoring cardiac fibrosis: a technical challenge, Neth Heart J, 20: 44-8.

de Lange, F. J., A. F. Moorman, R. H. Anderson, J. Manner, A. T. Soufan, C. de Gierde Vries, M. D. Schneider, S. Webb, M. J. van den Hoff, and V. M. Christoffels. 2004. Lineage and morphogenetic analysis of the cardiac valves, Circ Res, 95: 64554.

de Melker, A. A., and A. Sonnenberg. 1999. Integrins: alternative splicing as a mechanism to regulate ligand binding and integrin signaling events, Bioessays, 21: 499-509.

de Souza, R. R. 2002. Aging of myocardial collagen, Biogerontology, 3: 325-35.

DeQuach, J. A., V. Mezzano, A. Miglani, S. Lange, G. M. Keller, F. Sheikh, and K. L. Christman. 2010. Simple and high yielding method for preparing tissue specific extracellular matrix coatings for cell culture, PLoS One, 5: e13039.

Dettman, R. W., W. Denetclaw, Jr., C. P. Ordahl, and J. Bristow. 1998. Common epicardial origin of coronary vascular smooth muscle, perivascular fibroblasts, and intermyocardial fibroblasts in the avian heart, Dev Biol, 193: 169-81.

Development Core Team, R. 2009. R: a language and environment for statistical computing. R Foundation for Statistical Computing, Vienna.

Dhawan, L., B. Liu, B. C. Blaxall, and M. B. Taubman. 2007. A novel role for the glucocorticoid receptor in the regulation of monocyte chemoattractant protein-1 mRNA stability, J Biol Chem, 282: 10146-52.

Didangelos, A., X. Yin, K. Mandal, M. Baumert, M. Jahangiri, and M. Mayr. 2010. Proteomics characterization of extracellular space components in the human aorta, Mol Cell Proteomics, 9: 2048-62.

Didié, Michael, Peter Christalla, Michael Rubart, Vijayakumar Muppala, Stephan Döker, Bernhard Unsöld, Ali El-Armouche, Thomas Rau, Thomas Eschenhagen, 
Alexander P. Schwoerer, Heimo Ehmke, Udo Schumacher, Sigrid Fuchs, Claudia Lange, Alexander Becker, Wen Tao, John A. Scherschel, Mark H. Soonpaa, Tao Yang, Qiong Lin, Martin Zenke, Dong-Wook Han, Hans R. Schöler, Cornelia Rudolph, Doris Steinemann, Brigitte Schlegelberger, Steve Kattman, Alec Witty, Gordon Keller, Loren J. Field, and Wolfram-Hubertus Zimmermann. 2013. Parthenogenetic stem cells for tissue-engineered heart repair, The Journal of Clinical Investigation, 123: 1285-98.

Dobin, A., C. A. Davis, F. Schlesinger, J. Drenkow, C. Zaleski, S. Jha, P. Batut, M. Chaisson, and T. R. Gingeras. 2013. STAR: ultrafast universal RNA-seq aligner, Bioinformatics, 29: 15-21.

Doege, K. J., and J. H. Fessler. 1986. Folding of carboxyl domain and assembly of procollagen I, J Biol Chem, 261: 8924-35.

Domian, I. J., M. Chiravuri, P. van der Meer, A. W. Feinberg, X. Shi, Y. Shao, S. M. Wu, K. K. Parker, and K. R. Chien. 2009. Generation of functional ventricular heart muscle from mouse ventricular progenitor cells, Science, 326: 426-9.

Drogemuller, C., D. Becker, A. Brunner, B. Haase, P. Kircher, F. Seeliger, M. Fehr, U. Baumann, K. Lindblad-Toh, and T. Leeb. 2009. A missense mutation in the SERPINH1 gene in Dachshunds with osteogenesis imperfecta, PLoS Genet, 5: e1000579.

Du, A., J. M. Sanger, and J. W. Sanger. 2008. Cardiac myofibrillogenesis inside intact embryonic hearts, Dev Biol, 318: 236-46.

Durinck, S., P. T. Spellman, E. Birney, and W. Huber. 2009. Mapping identifiers for the integration of genomic datasets with the R/Bioconductor package biomaRt, Nat Protoc, 4: 1184-91.

Edmondson, R., J. J. Broglie, A. F. Adcock, and L. Yang. 2014. Three-dimensional cell culture systems and their applications in drug discovery and cell-based biosensors, Assay Drug Dev Technol, 12: 207-18.

Eghbali, M., O. O. Blumenfeld, S. Seifter, P. M. Buttrick, L. A. Leinwand, T. F. Robinson, M. A. Zern, and M. A. Giambrone. 1989. Localization of types I, III and IV collagen mRNAs in rat heart cells by in situ hybridization, J Mol Cell Cardiol, 21: 103-13.

Eghbali, Mahboubeh, Mark J. Czaja, Maria Zeydel, Francis R. Weiner, Mark A. Zern, Sam Seifter, and Olga O. Blumenfeld. 1988. Collagen chain mRNAs in isolated heart cells from young and adult rats, Journal of Molecular and Cellular Cardiology, 20: 267-76.

Eghbali, M., and K. T. Weber. 1990. Collagen and the myocardium: fibrillar structure, biosynthesis and degradation in relation to hypertrophy and its regression, Mol Cell Biochem, 96: 1-14.

Engler, A. J., C. Carag-Krieger, C. P. Johnson, M. Raab, H. Y. Tang, D. W. Speicher, J. W. Sanger, J. M. Sanger, and D. E. Discher. 2008. Embryonic cardiomyocytes beat best on a matrix with heart-like elasticity: scar-like rigidity inhibits beating, J Cell Sci, 


\section{1: $3794-802$.}

Eschenhagen, T., C. Fink, U. Remmers, H. Scholz, J. Wattchow, J. Weil, W. Zimmermann, H. H. Dohmen, H. Schafer, N. Bishopric, T. Wakatsuki, and E. L. Elson. 1997. Three-dimensional reconstitution of embryonic cardiomyocytes in a collagen matrix: a new heart muscle model system, Faseb j, 11: 683-94.

Fan, D., A. Takawale, J. Lee, and Z. Kassiri. 2012. Cardiac fibroblasts, fibrosis and extracellular matrix remodeling in heart disease, Fibrogenesis Tissue Repair, 5: 15.

Farhadian, F., F. Contard, A. Corbier, A. Barrieux, L. Rappaport, and J. L. Samuel. 1995. Fibronectin expression during physiological and pathological cardiac growth, $J$ Mol Cell Cardiol, 27: 981-90.

Fassler, R., and M. Meyer. 1995. Consequences of lack of beta 1 integrin gene expression in mice, Genes Dev, 9: 1896-908.

Fassler, R., J. Rohwedel, V. Maltsev, W. Bloch, S. Lentini, K. Guan, D. Gullberg, J. Hescheler, K. Addicks, and A. M. Wobus. 1996. Differentiation and integrity of cardiac muscle cells are impaired in the absence of beta 1 integrin, J Cell Sci, 109 ( Pt 13): 2989-99.

Favor, J., C. J. Gloeckner, D. Janik, M. Klempt, A. Neuhauser-Klaus, W. Pretsch, W. Schmahl, and L. Quintanilla-Fend. 2007. Type IV procollagen missense mutations associated with defects of the eye, vascular stability, the brain, kidney function and embryonic or postnatal viability in the mouse, Mus musculus: an extension of the Col4a1 allelic series and the identification of the first two Col4a2 mutant alleles, Genetics, 175: 725-36.

Feinberg, A. W., P. W. Alford, H. Jin, C. M. Ripplinger, A. A. Werdich, S. P. Sheehy, A. Grosberg, and K. K. Parker. 2012. Controlling the contractile strength of engineered cardiac muscle by hierarchal tissue architecture, Biomaterials, 33: 573241.

Fernandes, Hugo, Lorenzo Moroni, Clemens van Blitterswijk, and Jan de Boer. 2009. Extracellular matrix and tissue engineering applications, Journal of Materials Chemistry, 19: 5474-84.

Fletcher, D. A., and R. D. Mullins. 2010. Cell mechanics and the cytoskeleton, Nature, 463: 485-92.

Frangogiannis, N. G. 2012. Regulation of the inflammatory response in cardiac repair, Circ Res, 110: 159-73.

Fuegemann, C. J., A. K. Samraj, S. Walsh, B. K. Fleischmann, S. Jovinge, and M. Breitbach. 2010. Differentiation of mouse embryonic stem cells into cardiomyocytes via the hanging-drop and mass culture methods, Curr Protoc Stem Cell Biol, Chapter 1: Unit 1F.11.

Gaudesius, G., M. Miragoli, S. P. Thomas, and S. Rohr. 2003. Coupling of cardiac electrical activity over extended distances by fibroblasts of cardiac origin, Circ Res, 93: 421-8. 
Gelse, K., E. Poschl, and T. Aigner. 2003. Collagens--structure, function, and biosynthesis, Adv Drug Deliv Rev, 55: 1531-46.

Giancotti, F. G., and E. Ruoslahti. 1999. Integrin signaling, Science, 285: 1028-32.

Gilbert, H. F. 1997. Protein disulfide isomerase and assisted protein folding, J Biol Chem, 272: 29399-402.

Gittenberger-de Groot, A. C., M. P. Vrancken Peeters, M. M. Mentink, R. G. Gourdie, and R. E. Poelmann. 1998. Epicardium-derived cells contribute a novel population to the myocardial wall and the atrioventricular cushions, Circ Res, 82: 1043-52.

Goldsmith, E. C., A. D. Bradshaw, and F. G. Spinale. 2013. Cellular mechanisms of tissue fibrosis. 2. Contributory pathways leading to myocardial fibrosis: moving beyond collagen expression, Am J Physiol Cell Physiol, 304: C393-402.

Gregorio, C. C., and P. B. Antin. 2000. To the heart of myofibril assembly, Trends Cell Biol, 10: 355-62.

Griffith, L. G., and M. A. Swartz. 2006. Capturing complex 3D tissue physiology in vitro, Nat Rev Mol Cell Biol, 7: 211-24.

Grinnell, F. 1994. Fibroblasts, myofibroblasts, and wound contraction, J Cell Biol, 124: 401-4.

Grosberg, A., P. W. Alford, M. L. McCain, and K. K. Parker. 2011. Ensembles of engineered cardiac tissues for physiological and pharmacological study: heart on a chip, Lab Chip, 11: 4165-73.

Guan, K., D. O. Furst, and A. M. Wobus. 1999. Modulation of sarcomere organization during embryonic stem cell-derived cardiomyocyte differentiation, Eur J Cell Biol, 78: 813-23.

Gullberg, D., A. Tingstrom, A. C. Thuresson, L. Olsson, L. Terracio, T. K. Borg, and K. Rubin. 1990. Beta 1 integrin-mediated collagen gel contraction is stimulated by PDGF, Exp Cell Res, 186: 264-72.

Hagiwara, S., H. Iwasaka, S. Matsumoto, T. Noguchi, and H. Yoshioka. 2007. Coexpression of HSP47 gene and type I and type III collagen genes in LPS-induced pulmonary fibrosis in rats, Lung, 185: 31-7.

Hagiwara, S., H. Iwasaka, C. Shingu, S. Matumoto, A. Hasegawa, and T. Noguchi. 2011. Heat shock protein 47 (HSP47) antisense oligonucleotides reduce cardiac remodeling and improve cardiac function in a rat model of myocardial infarction, Thorac Cardiovasc Surg, 59: 386-92.

Harada, M., H. Itoh, O. Nakagawa, Y. Ogawa, Y. Miyamoto, K. Kuwahara, E. Ogawa, T. Igaki, J. Yamashita, I. Masuda, T. Yoshimasa, I. Tanaka, Y. Saito, and K. Nakao. 1997. Significance of ventricular myocytes and nonmyocytes interaction during cardiocyte hypertrophy: evidence for endothelin-1 as a paracrine hypertrophic factor from cardiac nonmyocytes, Circulation, 96: 3737-44. 
Harston, R. K., and D. Kuppuswamy. 2011. Integrins are the necessary links to hypertrophic growth in cardiomyocytes, J Signal Transduct, 2011: 521742.

Harvey, Adam, Ten-Yang Yen, Irina Aizman, Ciara Tate, and Casey Case. 2013. Proteomic Analysis of the Extracellular Matrix Produced by Mesenchymal Stromal Cells: Implications for Cell Therapy Mechanism, PLoS One, 8: e79283.

Higuchi, S., Q. Lin, J. Wang, T. K. Lim, S. B. Joshi, G. S. Anand, M. C. Chung, M. P. Sheetz, and H. Fujita. 2013. Heart extracellular matrix supports cardiomyocyte differentiation of mouse embryonic stem cells, J Biosci Bioeng, 115: 320-5.

Hill, J. A., and E. N. Olson. 2008. Cardiac plasticity, N Engl J Med, 358: 1370-80.

Hinton, R. B., Jr., J. Lincoln, G. H. Deutsch, H. Osinska, P. B. Manning, D. W. Benson, and K. E. Yutzey. 2006. Extracellular matrix remodeling and organization in developing and diseased aortic valves, Circ Res, 98: 1431-8.

Hirayoshi, K., H. Kudo, H. Takechi, A. Nakai, A. Iwamatsu, K. M. Yamada, and K. Nagata. 1991. HSP47: a tissue-specific, transformation-sensitive, collagen-binding heat shock protein of chicken embryo fibroblasts, Mol Cell Biol, 11: 4036-44.

Hornberger, L. K., S. Singhroy, T. Cavalle-Garrido, W. Tsang, F. Keeley, and M. Rabinovitch. 2000. Synthesis of extracellular matrix and adhesion through beta(1) integrins are critical for fetal ventricular myocyte proliferation, Circ Res, 87: 508-15.

Howard, C. M., and T. A. Baudino. 2014. Dynamic cell-cell and cell-ECM interactions in the heart, J Mol Cell Cardiol, 70: 19-26.

Hudson, J., D. Titmarsh, A. Hidalgo, E. Wolvetang, J. Cooper-White. 2012. Primitive cardiac cells from human embryonic stem cells, Stem Cells Dev, 21: 1513-23.

Hynes, R. O. 1987. Integrins: a family of cell surface receptors, Cell, 48: 549-54.

Hynes, R. O. 2002. Integrins: bidirectional, allosteric signaling machines, Cell, 110: 673-87.

Ieda, M., T. Tsuchihashi, K. N. Ivey, R. S. Ross, T. T. Hong, R. M. Shaw, and D. Srivastava. 2009. Cardiac fibroblasts regulate myocardial proliferation through beta1 integrin signaling, Dev Cell, 16: 233-44.

Ingber, D. E. 2006. Mechanical control of tissue morphogenesis during embryological development, Int J Dev Biol, 50: 255-66.

Irion, Stefan, Herve Luche, Paul Gadue, Hans Joerg Fehling, Marion Kennedy, and Gordon Keller. 2007. Identification and targeting of the ROSA26 locus in human embryonic stem cells, Nat Biotech, 25: 1477-82.

Ishida, Y., H. Kubota, A. Yamamoto, A. Kitamura, H. P. Bachinger, and K. Nagata. 2006. Type I collagen in Hsp47-null cells is aggregated in endoplasmic reticulum and deficient in N-propeptide processing and fibrillogenesis, Mol Biol Cell, 17: 2346-55.

Ishida, Y., and K. Nagata. 2011. Hsp47 as a collagen-specific molecular chaperone, 
Methods Enzymol, 499: 167-82.

Ishida, Y., A. Yamamoto, A. Kitamura, S. R. Lamande, T. Yoshimori, J. F. Bateman, H. Kubota, and K. Nagata. 2009. Autophagic elimination of misfolded procollagen aggregates in the endoplasmic reticulum as a means of cell protection, $\mathrm{Mol} \mathrm{Biol} \mathrm{Cell,}$ 20: $2744-54$.

Izzard, C. S., and L. R. Lochner. 1976. Cell-to-substrate contacts in living fibroblasts: an interference reflexion study with an evaluation of the technique, J Cell Sci, 21: 129-59.

Jacot, J. G., J. C. Martin, and D. L. Hunt. 2010. Mechanobiology of cardiomyocyte development, J Biomech, 43: 93-8.

Janicki, J. S., and G. L. Brower. 2002. The role of myocardial fibrillar collagen in ventricular remodeling and function, J Card Fail, 8: S319-25.

Jugdutt, B. I. 2003. Remodeling of the myocardium and potential targets in the collagen degradation and synthesis pathways, Curr Drug Targets Cardiovasc Haematol Disord, 3: 1-30.

Jugdutt, B. I. 2003. Ventricular remodeling after infarction and the extracellular collagen matrix: when is enough enough?, Circulation, 108: 1395-403.

Kakkar, R., and R. T. Lee. 2010. Intramyocardial fibroblast myocyte communication, Circ Res, 106: 47-57.

Kakugawa, T., H. Mukae, T. Hayashi, H. Ishii, K. Abe, T. Fujii, H. Oku, M. Miyazaki, J. Kadota, and S. Kohno. 2004. Pirfenidone attenuates expression of HSP47 in murine bleomycin-induced pulmonary fibrosis, Eur Respir J, 24: 57-65.

Kakugawa, T., H. Mukae, Y. Hishikawa, H. Ishii, N. Sakamoto, Y. Ishimatsu, T. Fujii, T. Koji, and S. Kohno. 2010. Localization of HSP47 mRNA in murine bleomycin-induced pulmonary fibrosis, Virchows Arch, 456: 309-15.

Kambe, K., A. Yamamoto, T. Yoshimori, K. Hirayoshi, R. Ogawa, and Y. Tashiro. 1994. Preferential localization of heat shock protein 47 in dilated endoplasmic reticulum of chicken chondrocytes, J Histochem Cytochem, 42: 833-41.

Karamifar, H., H. Ilkhanipoor, G. Ajami, Z. Karamizadeh, G. Amirhakimi, and A. M. Shakiba. 2013. Cardiovascular involvement in children with osteogenesis imperfecta, Iran J Pediatr, 23: 513-8.

Keller, R. S., S. Y. Shai, C. J. Babbitt, C. G. Pham, R. J. Solaro, M. L. Valencik, J. C. Loftus, and R. S. Ross. 2001. Disruption of integrin function in the murine myocardium leads to perinatal lethality, fibrosis, and abnormal cardiac performance, Am J Pathol, 158: 1079-90.

Kirchhofer, D., L. R. Languino, E. Ruoslahti, and M. D. Pierschbacher. 1990. Alpha 2 beta 1 integrins from different cell types show different binding specificities, $J$ Biol Chem, 265: 615-8. 
Kléber, André G., Michiel J. Janse, and Vladimir G. Fast. 2011. Normal and Abnormal Conduction in the Heart. in, Comprehensive Physiology (John Wiley \& Sons, Inc.).

Klewer, S. E., S. L. Krob, S. J. Kolker, and G. T. Kitten. 1998. Expression of type VI collagen in the developing mouse heart, Dev Dyn, 211: 248-55.

Klug, M. G., M. H. Soonpaa, G. Y. Koh, and L. J. Field. 1996. Genetically selected cardiomyocytes from differentiating embronic stem cells form stable intracardiac grafts, J Clin Invest, 98: 216-24.

Knight, C. G., L. F. Morton, A. R. Peachey, D. S. Tuckwell, R. W. Farndale, and M. J. Barnes. 2000. The collagen-binding A-domains of integrins alpha(1)beta(1) and alpha(2)beta(1) recognize the same specific amino acid sequence, GFOGER, in native (triple-helical) collagens, J Biol Chem, 275: 35-40.

Kofidis, T., P. Akhyari, J. Boublik, P. Theodorou, U. Martin, A. Ruhparwar, S. Fischer, T. Eschenhagen, H. P. Kubis, T. Kraft, R. Leyh, and A. Haverich. 2002. In vitro engineering of heart muscle: artificial myocardial tissue, J Thorac Cardiovasc Surg, 124: 63-9.

Kohl, P. 2003. Heterogeneous cell coupling in the heart: an electrophysiological role for fibroblasts, Circ Res, 93: 381-3.

Koide, T., S. Asada, Y. Takahara, Y. Nishikawa, K. Nagata, and K. Kitagawa. 2006. Specific recognition of the collagen triple helix by chaperone HSP47: minimal structural requirement and spatial molecular orientation, J Biol Chem, 281: 3432-8.

Koivu, J., R. Myllyla, T. Helaakoski, T. Pihlajaniemi, K. Tasanen, and K. I. Kivirikko. 1987. A single polypeptide acts both as the beta subunit of prolyl 4hydroxylase and as a protein disulfide-isomerase, J Biol Chem, 262: 6447-9.

Kolditz, D. P., M. C. Wijffels, N. A. Blom, A. van der Laarse, N. D. Hahurij, H. LieVenema, R. R. Markwald, R. E. Poelmann, M. J. Schalij, and A. C. Gittenberger-de Groot. 2008. Epicardium-derived cells in development of annulus fibrosis and persistence of accessory pathways, Circulation, 117: 1508-17.

Kolodney, M. S., and E. L. Elson. 1993. Correlation of myosin light chain phosphorylation with isometric contraction of fibroblasts, $J$ Biol Chem, 268: 23850-5.

Kovacic-Milivojevic, B., F. Roediger, E. A. Almeida, C. H. Damsky, D. G. Gardner, and D. Ilic. 2001. Focal adhesion kinase and p130Cas mediate both sarcomeric organization and activation of genes associated with cardiac myocyte hypertrophy, Mol Biol Cell, 12: 2290-307.

Krenning, G., E. M. Zeisberg, and R. Kalluri. 2010. The origin of fibroblasts and mechanism of cardiac fibrosis, J Cell Physiol, 225: 631-7.

Kruithof, B. P., S. A. Krawitz, and V. Gaussin. 2007. Atrioventricular valve development during late embryonic and postnatal stages involves condensation and extracellular matrix remodeling, Dev Biol, 302: 208-17. 
Krupnick, A. S., D. Kreisel, F. H. Engels, W. Y. Szeto, T. Plappert, S. H. Popma, A. W. Flake, and B. R. Rosengard. 2002. A novel small animal model of left ventricular tissue engineering, J Heart Lung Transplant, 21: 233-43.

Kuhn, B., F. del Monte, R. J. Hajjar, Y. S. Chang, D. Lebeche, S. Arab, and M. T. Keating. 2007. Periostin induces proliferation of differentiated cardiomyocytes and promotes cardiac repair, Nat Med, 13: 962-9.

Lamande, S. R., and J. F. Bateman. 1999. Procollagen folding and assembly: the role of endoplasmic reticulum enzymes and molecular chaperones, Semin Cell Dev Biol, 10: 455-64.

LaNasa, S. M., and S. J. Bryant. 2009. Influence of ECM proteins and their analogs on cells cultured on 2-D hydrogels for cardiac muscle tissue engineering, Acta Biomater, 5: 2929-38.

Lang, K., F. X. Schmid, and G. Fischer. 1987. Catalysis of protein folding by prolyl isomerase, Nature, 329: 268-70.

Langholz, O., D. Rockel, C. Mauch, E. Kozlowska, I. Bank, T. Krieg, and B. Eckes. 1995. Collagen and collagenase gene expression in three-dimensional collagen lattices are differentially regulated by alpha 1 beta 1 and alpha 2 beta 1 integrins, J Cell Biol, 131: 1903-15.

Larsen, M., V. V. Artym, J. A. Green, and K. M. Yamada. 2006. The matrix reorganized: extracellular matrix remodeling and integrin signaling, Curr Opin Cell Biol, 18: 463-71.

Lechat, Philippe, Milton Packer, Stephan Chalon, Michel Cucherat, Tarek Arab, and Jean-Pierre Boissel. 1998. Clinical Effects of $\beta$-Adrenergic Blockade in Chronic Heart Failure: A Meta-Analysis of Double-Blind, Placebo-Controlled, Randomized Trials, Circulation, 98: 1184-91.

Leor, J., S. Aboulafia-Etzion, A. Dar, L. Shapiro, I. M. Barbash, A. Battler, Y. Granot, and S. Cohen. 2000. Bioengineered cardiac grafts: A new approach to repair the infarcted myocardium?, Circulation, 102: Iii56-61.

Lesman, A., M. Habib, O. Caspi, A. Gepstein, G. Arbel, S. Levenberg, and L. Gepstein. 2010. Transplantation of a tissue-engineered human vascularized cardiac muscle, Tissue Eng Part A, 16: 115-25.

Li, A. H., P. P. Liu, F. J. Villarreal, and R. A. Garcia. 2014. Dynamic changes in myocardial matrix and relevance to disease: translational perspectives, Circ Res, 114: 916-27.

Li, R. K., Z. Q. Jia, R. D. Weisel, D. A. Mickle, A. Choi, and T. M. Yau. 1999. Survival and function of bioengineered cardiac grafts, Circulation, 100: Ii63-9.

Li, R. K., T. M. Yau, R. D. Weisel, D. A. Mickle, T. Sakai, A. Choi, and Z. Q. Jia. 2000. Construction of a bioengineered cardiac graft, J Thorac Cardiovasc Surg, 119: 368-75. 
Li, S., J. L. Guan, and S. Chien. 2005. Biochemistry and biomechanics of cell motility, Annu Rev Biomed Eng, 7: 105-50.

Liau, B., N. Christoforou, K. W. Leong, and N. Bursac. 2011. Pluripotent stem cellderived cardiac tissue patch with advanced structure and function, Biomaterials, 32: 9180-7.

Lie-Venema, H., N. M. van den Akker, N. A. Bax, E. M. Winter, S. Maas, T. Kekarainen, R. C. Hoeben, M. C. deRuiter, R. E. Poelmann, and A. C. Gittenbergerde Groot. 2007. Origin, fate, and function of epicardium-derived cells (EPDCs) in normal and abnormal cardiac development, ScientificWorldJournal, 7: 1777-98.

Lincoln, J., C. M. Alfieri, and K. E. Yutzey. 2004. Development of heart valve leaflets and supporting apparatus in chicken and mouse embryos, Dev Dyn, 230: 23950 .

Lindert, U., M. A. Weis, J. Rai, F. Seeliger, I. Hausser, T. Leeb, D. Eyre, M. Rohrbach, and C. Giunta. 2015. Molecular Consequences of the SERPINH1/HSP47 Mutation in the Dachshund Natural Model of Osteogenesis Imperfecta, J Biol Chem, 290: $17679-89$.

Liu, B., L. Dhawan, B. C. Blaxall, and M. B. Taubman. 2010. Protein kinase Cdelta mediates MCP-1 mRNA stabilization in vascular smooth muscle cells, Mol Cell Biochem, 344: 73-9.

Liu, H., B. Chen, and B. Lilly. 2008. Fibroblasts potentiate blood vessel formation partially through secreted factor TIMP-1, Angiogenesis, 11: 223-34.

Liu, X., H. Wu, M. Byrne, S. Krane, and R. Jaenisch. 1997. Type III collagen is crucial for collagen I fibrillogenesis and for normal cardiovascular development, Proc Natl Acad Sci U S A, 94: 1852-6.

Livak, K. J., and T. D. Schmittgen. 2001. Analysis of relative gene expression data using real-time quantitative PCR and the 2(-Delta Delta C(T)) Method, Methods, 25: 402-8.

Lo, S. H. 2006. Focal adhesions: whats new inside, Dev Biol, 294: 280-91.

Lockhart, M., E. Wirrig, A. Phelps, and A. Wessels. 2011. Extracellular matrix and heart development, Birth Defects Res A Clin Mol Teratol, 91: 535-50.

Lohler, J., R. Timpl, and R. Jaenisch. 1984. Embryonic lethal mutation in mouse collagen I gene causes rupture of blood vessels and is associated with erythropoietic and mesenchymal cell death, Cell, 38: 597-607.

Lompre, A. M., B. Nadal-Ginard, and V. Mahdavi. 1984. Expression of the cardiac ventricular alpha- and beta-myosin heavy chain genes is developmentally and hormonally regulated, J Biol Chem, 259: 6437-46.

Louault, C., N. Benamer, J. F. Faivre, D. Potreau, and J. Bescond. 2008. Implication of connexins 40 and 43 in functional coupling between mouse cardiac fibroblasts in primary culture, Biochim Biophys Acta, 1778: 2097-104. 
MacKenna, D., S. R. Summerour, and F. J. Villarreal. 2000. Role of mechanical factors in modulating cardiac fibroblast function and extracellular matrix synthesis, Cardiovasc Res, 46: 257-63.

Maitra, N., I. L. Flink, J. J. Bahl, and E. Morkin. 2000. Expression of alpha and beta integrins during terminal differentiation of cardiomyocytes, Cardiovasc Res, 47: 71525 .

Majkut, S., T. Idema, J. Swift, C. Krieger, A. Liu, and D. E. Discher. 2013. Heartspecific stiffening in early embryos parallels matrix and myosin expression to optimize beating, Curr Biol, 23: 2434-9.

Manso, A. M., S. M. Kang, and R. S. Ross. 2009. Integrins, focal adhesions, and cardiac fibroblasts, J Investig Med, 57: 856-60.

Masago, Y., A. Hosoya, K. Kawasaki, S. Kawano, A. Nasu, J. Toguchida, K. Fujita, H. Nakamura, G. Kondoh, and K. Nagata. 2012. The molecular chaperone Hsp47 is essential for cartilage and endochondral bone formation, J Cell Sci, 125: 1118-28.

Masuda, H., M. Fukumoto, K. Hirayoshi, and K. Nagata. 1994. Coexpression of the collagen-binding stress protein HSP47 gene and the alpha 1(I) and alpha 1(III) collagen genes in carbon tetrachloride-induced rat liver fibrosis, J Clin Invest, 94: 2481-8.

Matsuoka, Y., H. Kubota, E. Adachi, N. Nagai, T. Marutani, N. Hosokawa, and K. Nagata. 2004. Insufficient folding of type IV collagen and formation of abnormal basement membrane-like structure in embryoid bodies derived from Hsp47-null embryonic stem cells, Mol Biol Cell, 15: 4467-75.

Matsuura, K., S. Masuda, Y. Haraguchi, N. Yasuda, T. Shimizu, N. Hagiwara, P. W. Zandstra, and T. Okano. 2011. Creation of mouse embryonic stem cell-derived cardiac cell sheets, Biomaterials, 32: 7355-62.

Mays, P. K., R. J. McAnulty, J. S. Campa, and G. J. Laurent. 1991. Age-related changes in collagen synthesis and degradation in rat tissues. Importance of degradation of newly synthesized collagen in regulating collagen production, Biochem $J, 276$ ( Pt 2): 307-13.

Metsaranta, M., S. Garofalo, G. Decker, M. Rintala, B. de Crombrugghe, and E. Vuorio. 1992. Chondrodysplasia in transgenic mice harboring a 15-amino acid deletion in the triple helical domain of pro alpha 1(II) collagen chain, J Cell Biol, 118: 203-12.

Midwood. K., I. Wierzbicka-Patynowski, J. Schwarzbauer. 2002. Methods in CellMatrix Adhesion (Adams J. ed) pp. 145-161, Academic Press, San Diego.

Nag, A. C., and R. Zak. 1979. Dissociation of adult mammalian heart into single cell suspension: an ultrastructural study, J Anat, 129: 541-59.

Nagai, N., M. Hosokawa, S. Itohara, E. Adachi, T. Matsushita, N. Hosokawa, and K. Nagata. 2000. Embryonic lethality of molecular chaperone hsp47 knockout mice is associated with defects in collagen biosynthesis, J Cell Biol, 150: 1499-506. 
Nagata, K. 1996. Hsp47: a collagen-specific molecular chaperone, Trends Biochem Sci, 21: 22-6.

Nagata, K. 1998. Expression and function of heat shock protein 47: a collagenspecific molecular chaperone in the endoplasmic reticulum, Matrix Biol, 16: 379-86.

Nagata, K. 2003. HSP47 as a collagen-specific molecular chaperone: function and expression in normal mouse development, Semin Cell Dev Biol, 14: 275-82.

Nagata, K., and N. Hosokawa. 1996. Regulation and function of collagen-specific molecular chaperone, HSP47, Cell Struct Funct, 21: 425-30.

Nagata, K., S. Saga, and K. M. Yamada. 1986. A major collagen-binding protein of chick embryo fibroblasts is a novel heat shock protein, J Cell Biol, 103: 223-9.

Nagata, K., and K. M. Yamada. 1986. Phosphorylation and transformation sensitivity of a major collagen-binding protein of fibroblasts, J Biol Chem, 261: 7531-6.

Nagy, A., J. Rossant, R. Nagy, W. Abramow-Newerly, and J. C. Roder. 1993. Derivation of completely cell culture-derived mice from early-passage embryonic stem cells, Proc Natl Acad Sci U S A, 90: 8424-8.

Naito, H., I. Melnychenko, M. Didie, K. Schneiderbanger, P. Schubert, S. Rosenkranz, T. Eschenhagen, and W. H. Zimmermann. 2006. Optimizing engineered heart tissue for therapeutic applications as surrogate heart muscle, Circulation, 114: I72-8.

Nakai, A., K. Hirayoshi, and K. Nagata. 1990. Transformation of BALB/3T3 cells by simian virus 40 causes a decreased synthesis of a collagen-binding heat-shock protein (hsp47), J Biol Chem, 265: 992-9.

Nelson, C. M., and J. Tien. 2006. Microstructured extracellular matrices in tissue engineering and development, Curr Opin Biotechnol, 17: 518-23.

Nesvizhskii, Alexey I., Andrew Keller, Eugene Kolker, and Ruedi Aebersold. 2003. A Statistical Model for Identifying Proteins by Tandem Mass Spectrometry, Analytical Chemistry, 75: 4646-58.

Nichol, J. W., G. C. Engelmayr, Jr., M. Cheng, and L. E. Freed. 2008. Co-culture induces alignment in engineered cardiac constructs via MMP-2 expression, Biochem Biophys Res Commun, 373: 360-5.

Niebruegge, S., A. Nehring, H. Bar, M. Schroeder, R. Zweigerdt, and J. Lehmann. 2008. Cardiomyocyte production in mass suspension culture: embryonic stem cells as a source for great amounts of functional cardiomyocytes, Tissue Eng Part A, 14: 1591-601.

Norris, R. A., T. K. Borg, J. T. Butcher, T. A. Baudino, I. Banerjee, and R. R. Markwald. 2008. Neonatal and adult cardiovascular pathophysiological remodeling and repair: developmental role of periostin, Ann N Y Acad Sci, 1123: 30-40.

Norris, R. A., B. Damon, V. Mironov, V. Kasyanov, A. Ramamurthi, R. Moreno- 
Rodriguez, T. Trusk, J. D. Potts, R. L. Goodwin, J. Davis, S. Hoffman, X. Wen, Y. Sugi, C. B. Kern, C. H. Mjaatvedt, D. K. Turner, T. Oka, S. J. Conway, J. D. Molkentin, G. Forgacs, and R. R. Markwald. 2007. Periostin regulates collagen fibrillogenesis and the biomechanical properties of connective tissues, $J$ Cell Biochem, 101: 695-711.

Oberwallner, B., A. Brodarac, P. Anic, T. Saric, K. Wassilew, K. Neef, Y. H. Choi, and C. Stamm. 2015. Human cardiac extracellular matrix supports myocardial lineage commitment of pluripotent stem cells, Eur J Cardiothorac Surg, 47: 416-25; discussion 25 .

Olivey, H. E., N. A. Mundell, A. F. Austin, and J. V. Barnett. 2006. Transforming growth factor-beta stimulates epithelial-mesenchymal transformation in the proepicardium, Dev Dyn, 235: 50-9.

Oslowski, C. M., and F. Urano. 2011. Measuring ER stress and the unfolded protein response using mammalian tissue culture system, Methods Enzymol, 490: 71-92.

Packer, Milton, Michael R. Bristow, Jay N. Cohn, Wilson S. Colucci, Michael B. Fowler, Edward M. Gilbert, and Neil H. Shusterman. 1996. The Effect of Carvedilol on Morbidity and Mortality in Patients with Chronic Heart Failure, New England Journal of Medicine, 334: 1349-55.

Pampaloni, Francesco, Emmanuel G. Reynaud, and Ernst H. K. Stelzer. 2007. The third dimension bridges the gap between cell culture and live tissue, Nat Rev Mol Cell Biol, 8: 839-45.

Parker, K. K., and D. E. Ingber. 2007. Extracellular matrix, mechanotransduction and structural hierarchies in heart tissue engineering, Philos Trans R Soc Lond B Biol Sci, 362: 1267-79.

Patten, R. D., M. J. Aronovitz, L. Deras-Mejia, N. G. Pandian, G. G. Hanak, J. J. Smith, M. E. Mendelsohn, and M. A. Konstam. 1998. Ventricular remodeling in a mouse model of myocardial infarction, Am J Physiol, 274: H1812-20.

Perez-Pomares, J. M., A. Phelps, M. Sedmerova, R. Carmona, M. Gonzalez-Iriarte, R. Munoz-Chapuli, and A. Wessels. 2002. Experimental studies on the spatiotemporal expression of WT1 and RALDH2 in the embryonic avian heart: a model for the regulation of myocardial and valvuloseptal development by epicardially derived cells (EPDCs), Dev Biol, 247: 307-26.

Petit, V., and J. P. Thiery. 2000. Focal adhesions: structure and dynamics, Biol Cell, 92: 477-94.

Pfannkuche, K., S. Neuss, F. Pillekamp, L. P. Frenzel, W. Attia, T. Hannes, J. Salber, M. Hoss, M. Zenke, B. K. Fleischmann, J. Hescheler, and T. Saric. 2010. Fibroblasts facilitate the engraftment of embryonic stem cell-derived cardiomyocytes on threedimensional collagen matrices and aggregation in hanging drops, Stem Cells Dev, 19: 1589-99.

Pinto, A. R., A. Ilinykh, M. J. Ivey, J. T. Kuwabara, M. L. D'Antoni, R. Debuque, A. Chandran, L. Wang, K. Arora, N. A. Rosenthal, and M. D. Tallquist. 2016. Revisiting 
Cardiac Cellular Composition, Circ Res, 118: 400-9.

Plotnikov, S. V., and C. M. Waterman. 2013. Guiding cell migration by tugging, Curr Opin Cell Biol, 25: 619-26.

Pong, T., W. J. Adams, M. A. Bray, A. W. Feinberg, S. P. Sheehy, A. A. Werdich, and K. K. Parker. 2011. Hierarchical architecture influences calcium dynamics in engineered cardiac muscle, Exp Biol Med (Maywood), 236: 366-73.

Pontes Soares, C., V. Midlej, M. E. de Oliveira, M. Benchimol, M. L. Costa, and C. Mermelstein. 2012. 2D and 3D-organized cardiac cells shows differences in cellular morphology, adhesion junctions, presence of myofibrils and protein expression, PLoS One, 7: e38147.

Porter, K. E., and N. A. Turner. 2009. Cardiac fibroblasts: at the heart of myocardial remodeling, Pharmacol Ther, 123: 255-78.

Poschl, E., U. Schlotzer-Schrehardt, B. Brachvogel, K. Saito, Y. Ninomiya, and U. Mayer. 2004. Collagen IV is essential for basement membrane stability but dispensable for initiation of its assembly during early development, Development, 131: 1619-28.

Radisic, M., A. Marsano, R. Maidhof, Y. Wang, and G. Vunjak-Novakovic. 2008. Cardiac tissue engineering using perfusion bioreactor systems, Nat Protoc, 3: 719-38.

Radunovic, Z., L. L. Wekre, L. M. Diep, and K. Steine. 2011. Cardiovascular abnormalities in adults with osteogenesis imperfecta, Am Heart J, 161: 523-9.

Rahkonen, O., M. Su, H. Hakovirta, I. Koskivirta, S. G. Hormuzdi, E. Vuorio, P. Bornstein, and R. Penttinen. 2004. Mice with a deletion in the first intron of the Colla1 gene develop age-dependent aortic dissection and rupture, Circ Res, 94: 8390 .

Ross, R. S., and T. K. Borg. 2001. Integrins and the myocardium, Circ Res, 88: 11129 .

Samarel, A. M. 2005. Costameres, focal adhesions, and cardiomyocyte mechanotransduction, Am J Physiol Heart Circ Physiol, 289: H2291-301.

Sasaki, H., T. Sato, N. Yamauchi, T. Okamoto, D. Kobayashi, S. Iyama, J. Kato, T. Matsunaga, R. Takimoto, T. Takayama, K. Kogawa, N. Watanabe, and Y. Niitsu. 2002. Induction of heat shock protein 47 synthesis by TGF-beta and IL-1 beta via enhancement of the heat shock element binding activity of heat shock transcription factor 1, J Immunol, 168: 5178-83.

Sastry, S. K., and K. Burridge. 2000. Focal adhesions: a nexus for intracellular signaling and cytoskeletal dynamics, Exp Cell Res, 261: 25-36.

Satoh, M., K. Hirayoshi, S. Yokota, N. Hosokawa, and K. Nagata. 1996. Intracellular interaction of collagen-specific stress protein HSP47 with newly synthesized procollagen, J Cell Biol, 133: 469-83. 
Sauk, J. J., N. Nikitakis, and H. Siavash. 2005. Hsp47 a novel collagen binding serpin chaperone, autoantigen and therapeutic target, Front Biosci, 10: 107-18.

Sauk, J. J., T. Smith, K. Norris, and L. Ferreira. 1994. Hsp47 and the translationtranslocation machinery cooperate in the production of alpha 1(I) chains of type I procollagen, J Biol Chem, 269: 3941-6.

Schiro, J. A., B. M. Chan, W. T. Roswit, P. D. Kassner, A. P. Pentland, M. E. Hemler, A. Z. Eisen, and T. S. Kupper. 1991. Integrin alpha 2 beta 1 (VLA-2) mediates reorganization and contraction of collagen matrices by human cells, Cell, 67: 403-10.

Schwartz, M. A., and M. H. Ginsberg. 2002. Networks and crosstalk: integrin signalling spreads, Nat Cell Biol, 4: E65-8.

Schwartz, M. A., M. D. Schaller, and M. H. Ginsberg. 1995. Integrins: emerging paradigms of signal transduction, Annu Rev Cell Dev Biol, 11: 549-99.

Sengbusch, J. K., W. He, K. A. Pinco, and J. T. Yang. 2002. Dual functions of [alpha]4[beta]1 integrin in epicardial development: initial migration and long-term attachment, J Cell Biol, 157: 873-82.

Shai, S. Y., A. E. Harpf, C. J. Babbitt, M. C. Jordan, M. C. Fishbein, J. Chen, M. Omura, T. A. Leil, K. D. Becker, M. Jiang, D. J. Smith, S. R. Cherry, J. C. Loftus, and R. S. Ross. 2002. Cardiac myocyte-specific excision of the beta1 integrin gene results in myocardial fibrosis and cardiac failure, Circ Res, 90: 458-64.

Sheehy, S. P., A. Grosberg, and K. K. Parker. 2012. The contribution of cellular mechanotransduction to cardiomyocyte form and function, Biomech Model Mechanobiol, 11: 1227-39.

Shimazaki, M., K. Nakamura, I. Kii, T. Kashima, N. Amizuka, M. Li, M. Saito, K. Fukuda, T. Nishiyama, S. Kitajima, Y. Saga, M. Fukayama, M. Sata, and A. Kudo. 2008. Periostin is essential for cardiac healing after acute myocardial infarction, $J$ Exp Med, 205: 295-303.

Shimizu, T., M. Yamato, Y. Isoi, T. Akutsu, T. Setomaru, K. Abe, A. Kikuchi, M. Umezu, and T. Okano. 2002. Fabrication of pulsatile cardiac tissue grafts using a novel 3-dimensional cell sheet manipulation technique and temperature-responsive cell culture surfaces, Circ Res, 90: e40.

Simpson, D. G., M. Majeski, T. K. Borg, and L. Terracio. 1999. Regulation of cardiac myocyte protein turnover and myofibrillar structure in vitro by specific directions of stretch, Circ Res, 85: e59-69.

Simpson, P., and S. Savion. 1982. Differentiation of rat myocytes in single cell cultures with and without proliferating nonmyocardial cells. Cross-striations, ultrastructure, and chronotropic response to isoproterenol, Circ Res, 50: 101-16.

Smith, P. K., R. I. Krohn, G. T. Hermanson, A. K. Mallia, F. H. Gartner, M. D. Provenzano, E. K. Fujimoto, N. M. Goeke, B. J. Olson, and D. C. Klenk. 1985. Measurement of protein using bicinchoninic acid, Anal Biochem, 150: 76-85. 
Snider, P., K. N. Standley, J. Wang, M. Azhar, T. Doetschman, and S. J. Conway. 2009. Origin of cardiac fibroblasts and the role of periostin, Circ Res, 105: 934-47.

Soong, P. L., M. Tiburcy, and W. H. Zimmermann. 2012. Cardiac differentiation of human embryonic stem cells and their assembly into engineered heart muscle, Curr Protoc Cell Biol, Chapter 23: Unit23.8.

Souders, C. A., S. L. Bowers, and T. A. Baudino. 2009. Cardiac fibroblast: the renaissance cell, Circ Res, 105: 1164-76.

Stevens, K. R., K. L. Kreutziger, S. K. Dupras, F. S. Korte, M. Regnier, V. Muskheli, M. B. Nourse, K. Bendixen, H. Reinecke, and C. E. Murry. 2009. Physiological function and transplantation of scaffold-free and vascularized human cardiac muscle tissue, Proceedings of the National Academy of Sciences of the United States of America, 106: 16568-73.

Sussman, M. A., S. Welch, A. Walker, R. Klevitsky, T. E. Hewett, R. L. Price, E. Schaefer, and K. Yager. 2000. Altered focal adhesion regulation correlates with cardiomyopathy in mice expressing constitutively active rac1, J Clin Invest, 105: 87586.

Swynghedauw, B. 1999. Molecular mechanisms of myocardial remodeling, Physiol Rev, 79: 215-62.

Taber, L. A. 2001. Biomechanics of cardiovascular development, Annu Rev Biomed Eng, 3: 1-25.

Taguchi, T., and M. S. Razzaque. 2007. The collagen-specific molecular chaperone HSP47: is there a role in fibrosis?, Trends Mol Med, 13: 45-53.

Takechi, H., K. Hirayoshi, A. Nakai, H. Kudo, S. Saga, and K. Nagata. 1992. Molecular cloning of a mouse 47-kDa heat-shock protein (HSP47), a collagen-binding stress protein, and its expression during the differentiation of F9 teratocarcinoma cells, Eur J Biochem, 206: 323-9.

Takeda, N., and I. Manabe. 2011. Cellular Interplay between Cardiomyocytes and Nonmyocytes in Cardiac Remodeling, Int J Inflam, 2011: 535241.

Tasab, M., M. R. Batten, and N. J. Bulleid. 2000. Hsp47: a molecular chaperone that interacts with and stabilizes correctly-folded procollagen, EMBO J, 19: 2204-11.

Tasab, M., L. Jenkinson, and N. J. Bulleid. 2002. Sequence-specific recognition of collagen triple helices by the collagen-specific molecular chaperone HSP47, J Biol Chem, 277: 35007-12.

Terracio, L., D. Gullberg, K. Rubin, S. Craig, and T. K. Borg. 1989. Expression of collagen adhesion proteins and their association with the cytoskeleton in cardiac myocytes, Anat Rec, 223: 62-71.

Terracio, L., K. Rubin, D. Gullberg, E. Balog, W. Carver, R. Jyring, and T. K. Borg. 1991. Expression of collagen binding integrins during cardiac development and hypertrophy, Circ Res, 68: 734-44. 
Thomson, C. A., R. Tenni, and V. S. Ananthanarayanan. 2003. Mapping Hsp47 binding site(s) using CNBr peptides derived from type I and type II collagen, Protein Sci, 12: 1792-800.

Tiburcy, M., M. Didie, O. Boy, P. Christalla, S. Doker, H. Naito, B. C. Karikkineth, A. El-Armouche, M. Grimm, M. Nose, T. Eschenhagen, A. Zieseniss, D. M. Katschinksi, N. Hamdani, W. A. Linke, X. Yin, M. Mayr, and W. H. Zimmermann. 2011. Terminal differentiation, advanced organotypic maturation, and modeling of hypertrophic growth in engineered heart tissue, Circ Res, 109: 1105-14.

Tiburcy, M., T. Meyer, P. L. Soong, and W. H. Zimmermann. 2014. Collagen-based engineered heart muscle, Methods Mol Biol, 1181: 167-76.

Tobita, K., E. A. Schroder, J. P. Tinney, J. B. Garrison, and B. B. Keller. 2002. Regional passive ventricular stress-strain relations during development of altered loads in chick embryo, Am J Physiol Heart Circ Physiol, 282: H2386-96.

Tohyama, S., F. Hattori, M. Sano, T. Hishiki, Y. Nagahata, T. Matsuura, H. Hashimoto, T. Suzuki, H. Yamashita, Y. Satoh, T. Egashira, T. Seki, N. Muraoka, H. Yamakawa, Y. Ohgino, T. Tanaka, M. Yoichi, S. Yuasa, M. Murata, M. Suematsu, and K. Fukuda. 2013. Distinct metabolic flow enables large-scale purification of mouse and human pluripotent stem cell-derived cardiomyocytes, Cell Stem Cell, 12: 127-37.

Tulloch, N. L., V. Muskheli, M. V. Razumova, F. S. Korte, M. Regnier, K. D. Hauch, L. Pabon, H. Reinecke, and C. E. Murry. 2011. Growth of engineered human myocardium with mechanical loading and vascular coculture, Circ Res, 109: 47-59.

Turoverova, L. V., M. G. Khotin, N. M. Yudintseva, K. E. Magnusson, M. I. Blinova, G. P. Pinaev, and D. G. Tentler. 2009. Analysis of extracellular matrix proteins produced by cultured cells, Cell and Tissue Biology, 3: 497-502.

Uzawa, K., W. J. Grzesik, T. Nishiura, S. A. Kuznetsov, P. G. Robey, D. A. Brenner, and M. Yamauchi. 1999. Differential expression of human lysyl hydroxylase genes, lysine hydroxylation, and cross-linking of type I collagen during osteoblastic differentiation in vitro, J Bone Miner Res, 14: 1272-80.

Van der Loop, F. T., G. J. Van Eys, G. Schaart, and F. C. Ramaekers. 1996. Titin expression as an early indication of heart and skeletal muscle differentiation in vitro. Developmental re-organisation in relation to cytoskeletal constituents, J Muscle Res Cell Motil, 17: 23-36.

Van Duyn Graham, L., M. T. Sweetwyne, M. A. Pallero, and J. E. Murphy-Ullrich. 2010. Intracellular calreticulin regulates multiple steps in fibrillar collagen expression, trafficking, and processing into the extracellular matrix, J Biol Chem, 285: 7067-78.

van Rooij, E., L. B. Sutherland, J. E. Thatcher, J. M. DiMaio, R. H. Naseem, W. S. Marshall, J. A. Hill, and E. N. Olson. 2008. Dysregulation of microRNAs after myocardial infarction reveals a role of miR-29 in cardiac fibrosis, Proc Natl Acad Sci US A, 105: 13027-32. 
van Spreeuwel, A. C. C, N. A. M. Bax, C. V. C.Bouten. 2014. The relevance of extracellular matrix structure and composition in engineering the diseased cardiac microenvironment, OA Tissue Engineering, 18; 2(1):2.

Vandenburgh, H. H., and P. Karlisch. 1989. Longitudinal growth of skeletal myotubes in vitro in a new horizontal mechanical cell stimulator, In Vitro Cell Dev Biol, 25: 607-16.

Von der Mark, K. 1981. Localization of collagen types in tissues, Int Rev Connect Tissue Res, 9: 265-324.

Wang, Chih-Chen, and Chen-Lu Tsou. 1998. Enzymes as chaperones and chaperones as enzymes, FEBS Letters, 425: 382-84.

Warnes, Gregory R, Ben Bolker, L Bonebakker, R Gentleman, W Huber, A Liaw, T Lumley, M Maechler, A Magnusson, and S Moeller. 2009. gplots: Various R programming tools for plotting data, $R$ package version, 2 .

Weber, K. T. 1989. Cardiac interstitium in health and disease: the fibrillar collagen network, J Am Coll Cardiol, 13: 1637-52.

Wells, R. G. 2008. The role of matrix stiffness in regulating cell behavior, Hepatology, 47: 1394-400.

Wenstrup, R. J., J. B. Florer, E. W. Brunskill, S. M. Bell, I. Chervoneva, and D. E. Birk. 2004. Type V collagen controls the initiation of collagen fibril assembly, J Biol Chem, 279: 53331-7.

Widmer, C., J. M. Gebauer, E. Brunstein, S. Rosenbaum, F. Zaucke, C. Drogemuller, T. Leeb, and U. Baumann. 2012. Molecular basis for the action of the collagenspecific chaperone Hsp47/SERPINH1 and its structure-specific client recognition, Proc Natl Acad Sci U S A, 109: 13243-7.

Wobus, A. M., G. Wallukat, and J. Hescheler. 1991. Pluripotent mouse embryonic stem cells are able to differentiate into cardiomyocytes expressing chronotropic responses to adrenergic and cholinergic agents and $\mathrm{Ca} 2+$ channel blockers, Differentiation, 48: 173-82.

Xin, M., E. N. Olson, and R. Bassel-Duby. 2013. Mending broken hearts: cardiac development as a basis for adult heart regeneration and repair, Nat Rev Mol Cell Biol, 14: 529-41.

Yamamoto, N., T. Kinoshita, N. Nohata, H. Yoshino, T. Itesako, L. Fujimura, A. Mitsuhashi, H. Usui, H. Enokida, M. Nakagawa, M. Shozu, and N. Seki. 2013. Tumor-suppressive microRNA-29a inhibits cancer cell migration and invasion via targeting HSP47 in cervical squamous cell carcinoma, Int J Oncol, 43: 1855-63.

Yin, L., H. Bien, and E. Entcheva. 2004. Scaffold topography alters intracellular calcium dynamics in cultured cardiomyocyte networks, Am J Physiol Heart Circ Physiol, 287: H1276-85.

Young, J. L., and A. J. Engler. 2011. Hydrogels with time-dependent material 
properties enhance cardiomyocyte differentiation in vitro, Biomaterials, 32: 1002-9.

Zeisberg, E. M., and R. Kalluri. 2010. Origins of cardiac fibroblasts, Circ Res, 107: 1304-12.

Zeisberg, E. M., O. Tarnavski, M. Zeisberg, A. L. Dorfman, J. R. McMullen, E. Gustafsson, A. Chandraker, X. Yuan, W. T. Pu, A. B. Roberts, E. G. Neilson, M. H. Sayegh, S. Izumo, and R. Kalluri. 2007. Endothelial-to-mesenchymal transition contributes to cardiac fibrosis, Nat Med, 13: 952-61.

Zhang, D., I. Y. Shadrin, J. Lam, H. Q. Xian, H. R. Snodgrass, and N. Bursac. 2013. Tissue-engineered cardiac patch for advanced functional maturation of human ESCderived cardiomyocytes, Biomaterials, 34: 5813-20.

Zhu, J., G. Xiong, H. Fu, B. M. Evers, B. P. Zhou, and R. Xu. 2015. Chaperone Hsp47 Drives Malignant Growth and Invasion by Modulating an ECM Gene Network, Cancer Res, 75: 1580-91.

Zimmermann, W. H., C. Fink, D. Kralisch, U. Remmers, J. Weil, and T. Eschenhagen. 2000. Three-dimensional engineered heart tissue from neonatal rat cardiac myocytes, Biotechnol Bioeng, 68: 106-14.

Zimmermann, W. H., Didie, M., Wasmeier, G.H., Nixdorff, U., Hess, A., Melnychenko, I., Boy, O., Neuhuber, W.L., Weyand, M., and Eschenhagen, T. Cardiac grafting of engineered heart tissue in syngenic rats. Circulation. 2002;106:I151-157.

Zimmermann, W.H., J. Hudson, and M. Tiburcy. 2015. "A method to direct differentiation of pluripotent stem cells into functional heart muscle." In.: Google Patents. 


\section{Appendix}

\section{A1. Supplement data}

A

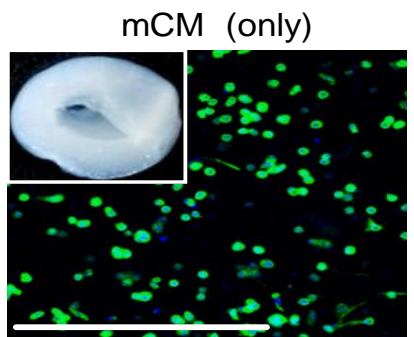

B

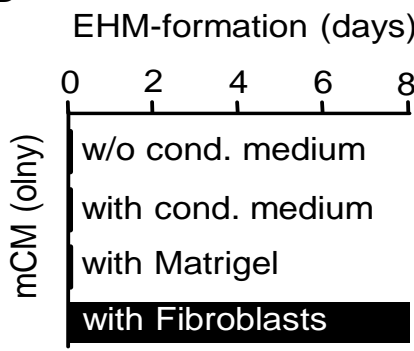

C

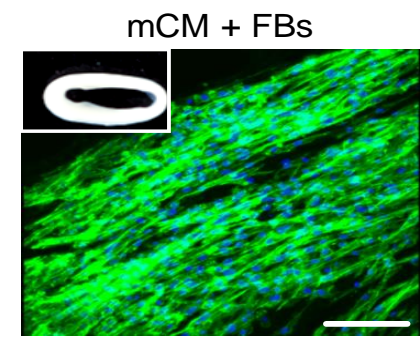

D

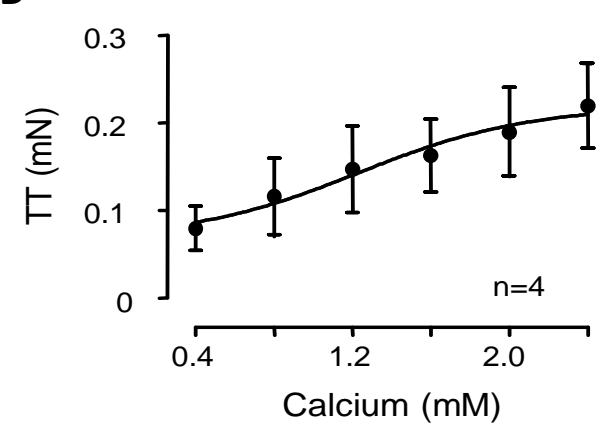

$\mathbf{E}$

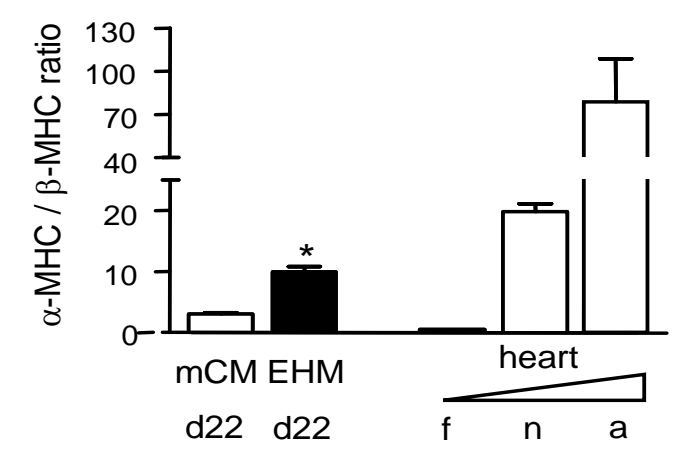

Figure S1. Advanced mCM maturation in cFB-supplemented EHMs. (A) EHMs generated with mCMs formed a spongy-like tissue with immature $\mathrm{mCMs}$ ( $\mathrm{n}=35$ in total). Single CMs stayed isolated with no tendency to form a functional syncytium, as indicated by the rounded shaped CMs ( $\alpha$-actinin: green). (B) The lack of tissue formation could not be rescued by using FB-conditioned medium or additional supplementation of growth factors and matrix components via Matrigel $(n=8)$. (C) Supplementation of cFBs ( $25 \%$ of total EHM composition) facilitated the formation of the typical EHM ring structure ( $\mathrm{n}=67$ in total). $\mathrm{mCMs}$ developed into tightly packed and longitudinally orientated muscle bundles. (D) Forces of contraction from cFB-supplemented EHMs, analysed by concentration response measurements under increasing extracellular $\mathrm{Ca}^{2+}(\mathrm{n}=4)$. (E) qPCR analysis of $\alpha$-MHC to $\beta$ MHC transcript ratio in 2D differentiated and 3D (EHM) matured mCMs of the same age (day 22). In addition, heart samples from fetal (f), neonatal (n) and adult (a) mice were analysed. *p $<0.05$ EHM (3D matured) vs. (2D matured; n=4/group). Green: F-Actin; Scale bars: $20 \mu \mathrm{m}$. (Data taken from Christalla et al., unpublished data). 


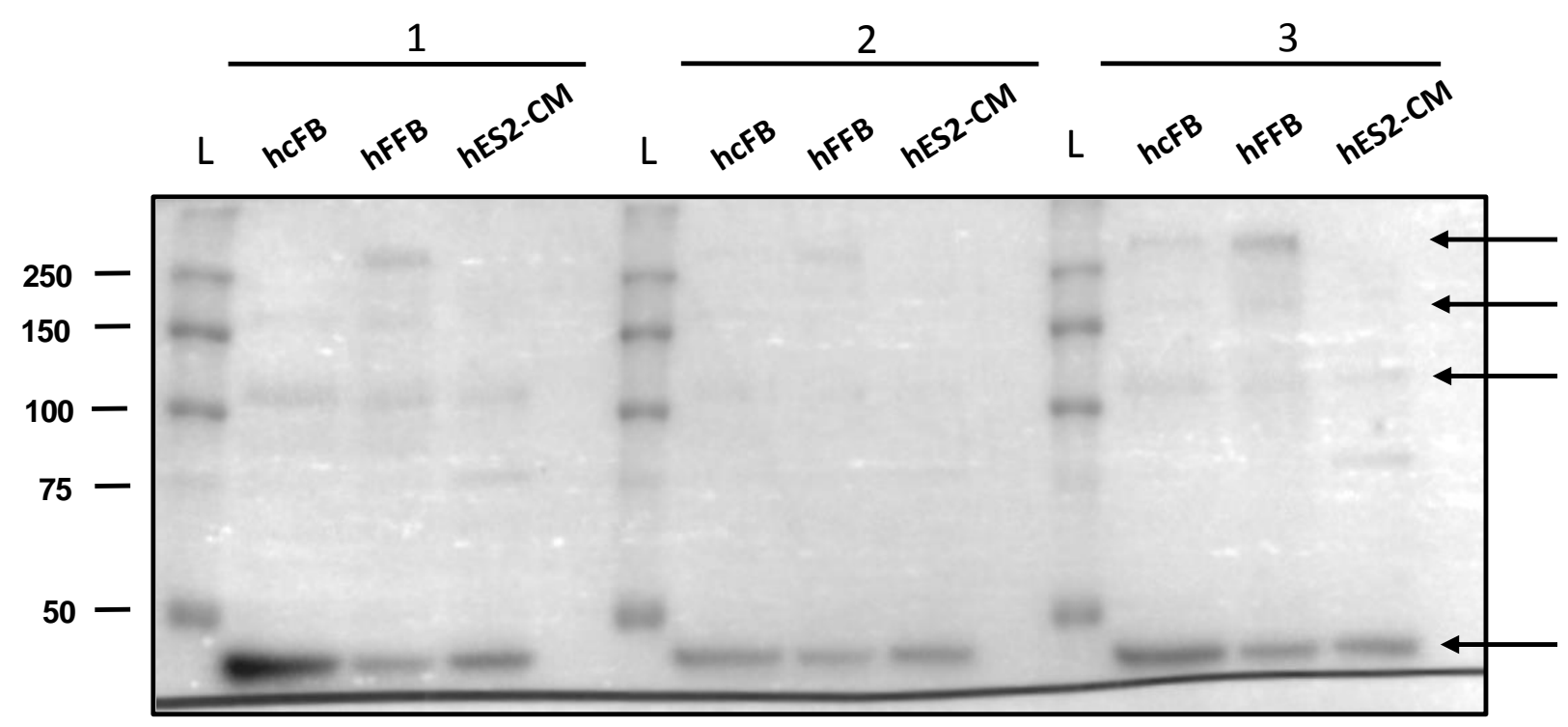

Figure S2. Validation of Anti-Integrin alpha 1 antibody for immunoblotting analysis in human samples. The Rabbit Polyclonal Anti-Integrin alpha 1 antibody (ab106267; Abcam) was tested on human FB populations (hcFB and hFFB) and purely enriched hCM populations (15ug sample; $\mathrm{n}=3$ /group), according to the manufacturer's recommended concentration and conditions. The predicted molecular weight band size of ITGA1 is $131 \mathrm{kDa}$ (according to the manufacturer's information), however immunoblotting analysis revealed non-specific bands in all samples. Strong signals were detected at around $40 \mathrm{kDa}$, in addition to fainter bands between; $100-125 \mathrm{kDa}$, around $180 \mathrm{kDa}$, and above $250 \mathrm{KDa}$. 'L': Protein molecular weight ladder. 


\section{A2. Reagents and medium}

\section{Buffers for neonatal rat heart isolation:}

For making CBFHH solution, the following stock solutions were prepared:

$\begin{array}{llll}\mathrm{NaCl} & 200 \mathrm{~g} / \mathrm{L} & \text { autoclaved } & 58.44 \mathrm{~g} / \mathrm{mol} \\ \mathrm{KCl} & 40 \mathrm{~g} / \mathrm{L} & \text { autoclaved } & 74.56 \mathrm{~g} / \mathrm{mol} \\ \mathrm{MgSO}_{4} \times \mathrm{H} 2 \mathrm{O} & 20 \mathrm{~g} / \mathrm{L} & \text { autoclaved } & 246.48 \mathrm{~g} / \mathrm{mol} \\ \mathrm{KH}_{2} \mathrm{PO}_{4} & 6 \mathrm{~g} / \mathrm{L} & \text { autoclaved } & 136.09 \mathrm{~g} / \mathrm{mol} \\ \mathrm{Na}_{2} \mathrm{HPO}_{4} \times 2 \mathrm{H}_{2} \mathrm{O} & 5.97 \mathrm{~g} / \mathrm{L} & \text { autoclaved } & 177.99 \mathrm{~g} / \mathrm{mol} \\ \text { Glucose (dehydrated) } & 100 \mathrm{~g} / \mathrm{L} & \text { filtered } & 180.16 \mathrm{~g} / \mathrm{mol} \\ \text { HEPES (Sigma) } & 47.66 \mathrm{~g} / \mathrm{L} & \text { filtered } & 238.3 \mathrm{~g} / \mathrm{mol}\end{array}$

$\underline{\text { Calcium and Bicarbonate-free Hanks with HEPES (CBFHH)-buffer solution }}$

\begin{tabular}{|c|c|}
\hline $810 \mathrm{ml}$ & $\mathrm{H}_{2} \mathrm{O}$ (sterile) \\
\hline $40 \mathrm{ml}$ & $\mathrm{NaCl}$ (stock) \\
\hline $10 \mathrm{ml}$ & $\mathrm{KCl}$ (stock) \\
\hline $10 \mathrm{ml}$ & $\mathrm{MgSO}_{4} \times \mathrm{H}_{2} \mathrm{O}$ (stock) \\
\hline $10 \mathrm{ml}$ & $\mathrm{KH}_{2} \mathrm{PO}_{4}$ (stock) \\
\hline $10 \mathrm{ml}$ & $\mathrm{Na}_{2} \mathrm{HPO}_{4} \times 2 \mathrm{H}_{2} \mathrm{O}$ (stock) \\
\hline $10 \mathrm{ml}$ & Glucose (stock) \\
\hline $100 \mathrm{ml}$ & HEPES (stock) \\
\hline
\end{tabular}

The $\mathrm{pH}$ was adjusted to 7.4 with $1 \mathrm{~mol} / \mathrm{L} \mathrm{NaOH}$, sterile filtered $(0,22 \mu \mathrm{m})$ and stored at $4{ }^{\circ} \mathrm{C}$, until use. 
$\underline{\text { Trypsin-stock solution }(50 \mathrm{X})}$

Dissolve trypsin (Trypsin certified 1:250 crude, Difco $^{\mathrm{TM}}$, BD Biosciences, \#0153-159; $100 \mathrm{~g})$ in CBFHH $(100 \mathrm{mg} / \mathrm{ml})$. Concentrated stocks were sterile filtered $(0.22$ $\mu \mathrm{m})$, aliquoted and stored at $-20{ }^{\circ} \mathrm{C}$.

DNase I-stock solution

DNase I,type V (from bovine spleen, Sigma \#D8764) was dissolved in sterile $\mathrm{H}_{2} \mathrm{O}$ (1 $\mathrm{mg} / \mathrm{ml})$. Concentrated stocks were sterile filtered $(0.22 \mu \mathrm{m})$, aliquoted and stored at $20^{\circ} \mathrm{C}$. DNase I stock was kept on ice after thawing.

$\underline{\text { Trypsin-working solution }}$

$0.5 \mathrm{ml} \quad \mathrm{P} / \mathrm{S}$ (100X; Gibco)

$1.3 \mathrm{ml} \quad$ Trypsin stock (50X)

$0.2 \mathrm{ml} \quad$ DNase I stock

Fill to $50 \mathrm{ml}$ with CBFHH solution.

$\underline{\text { DNase-working solutions }}$

$0.5 \mathrm{ml} \quad \mathrm{P} / \mathrm{S}(100 \mathrm{X}$; Gibco)

$1.7 \mathrm{ml} \quad$ Fetal Bovine Serum (FBS) (Gibco, 10270)

$0.2 \mathrm{ml} \quad$ DNase I stock

$0.5 \mathrm{ml} \quad$ Trypsin stock (50X)

Fill to $50 \mathrm{ml}$ with CBFHH solution. 


\section{$\underline{\text { Reagents and medium for cell culture: }}$}

Accutase digestion solution

Accutase solution (Millipore, SCR005)

$0.125 \%$ Trypsin (1:20 dilution from Trypsin $2.5 \%$ stock [Gibco, 15090-046])

$20 \mu \mathrm{g} / \mathrm{ml}$ DNase I

\section{$\underline{\text { Activin A stock solution }}$}

Dissolve Activin A (338-AC, R\&D systems) according to manufactures protocol to obtain a stock concentration of $10 \mu \mathrm{g} / \mathrm{ml}$.

\section{bFGF stock solution}

Dissolve bFGF (130-093-841; Stemgent) according to manufacturer's protocol to obtain a stock concentration of $10 \mu \mathrm{g} / \mathrm{ml}$. Aliquot into sterile microfuge tubes and store at $-20{ }^{\circ} \mathrm{C}$.

\section{$\underline{\text { BMP4 stock solution }}$}

Dissolve BMP4 (314-BP, R\&D systems) according to manufactures protocol to obtain a stock concentration of $10 \mu \mathrm{g} / \mathrm{ml}$.

\section{Cardiac Specification medium}

Serum free monolayer differentiation medium

\section{$5 \mu \mathrm{mol} / \mathrm{L}$ IWP4}

Medium containing grow factor supplements are prepared fresh, prior to use.

\section{$\underline{\text { CHIR stock solution }}$}

Dissolve CHIR (04-0004, Stemgent) in DMSO to obtain a stock concentration of 10 $\mathrm{mmol} / \mathrm{L}$.

\section{Collagenase I solution}

$100 \mathrm{mg}$ of collagenase I dissolved in $40 \mathrm{ml}$ of 1 X DPBS (containing $\mathrm{Ca}^{2+} / \mathrm{Mg}^{2+}$ ) and $10 \mathrm{ml}$ FBS (10270 Gibco) . Stock solution was sterile filtered, aliquoted and store at $20{ }^{\circ} \mathrm{C}$. 


\section{Differentiation medium}

$500 \mathrm{ml}$ Iscove's MEM (F0465, Biochrom)

$120 \mathrm{ml} \mathrm{FBS}$

$6 \mathrm{ml}$ L-Glutamine

$6 \mathrm{ml}$ MEM-NEAA

$6 \mathrm{ml} \mathrm{P} / \mathrm{S}$

$4.34 \mu 12-\beta-M e r c a p t o e t h a n o l$

$0.2 \mathrm{mM}$ (43 mg) L-ascorbic acid 2-phosphate salt (Sigma, A8960).

EDTA Solution $(0.5 \mathrm{mmol} / \mathrm{L})$

Add $500 \mu$ of the $0.5 \mathrm{~mol} / \mathrm{L}$ EDTA stock solution (pH 8.0), to $500 \mathrm{ml}$ of $1 \mathrm{X}$ PBS 14190; Invitrogen), containing $0.45 \mathrm{~g} \mathrm{NaCl}$. Sterile filter $(0.22 \mathrm{um})$, aliquot and store at $4{ }^{\circ} \mathrm{C}$ for 6 months.

Freezing medium

$90 \%$ FBS

$10 \%$ DMSO (276855, Sigma Aldrich)

Cell pellets were resuspended in FBS, followed by the addition of DMSO. Cell suspensions were distributed into cryovial (ml/vial and transferred into a isopropanol insulated container, prior to cryopreservation at either -80 or $-152{ }^{\circ} \mathrm{C}$.

$\underline{\text { HFF medium }}$

$500 \mathrm{ml}$ DMEM (42430, Invitrogen)

$88.8 \mathrm{ml}$ FBS

$3 \mathrm{ml} \mathrm{P} / \mathrm{S}$

$\underline{\text { hESC medium }}$

$500 \mathrm{ml}$ KO-DMEM (10829-018, Invitrogen)

$128.2 \mathrm{ml} \mathrm{KOSR}$ (10828028, Invitrogen)

$6.4 \mathrm{ml} \mathrm{L}$-glutamine

$6.4 \mathrm{ml}$ MEM NEAA

Add $10 \mu \mathrm{l}$ of bFGF stock solution into $10 \mathrm{ml}$ of hESC medium (final bFGF concentration: $10 \mathrm{ng} / \mathrm{ml}$ ) per $100 \mathrm{~mm}$ hESC culture plate. 


\section{$\underline{\text { IWR1 stock solution }}$}

Dissolve IWR1 (I0161, Sigma) in DMSO to obtain a stock concentration of 5 $\mathrm{mmol} / \mathrm{L}$.

\section{IWP4 stock solution}

Dissolve IWP4 (04-0036, Stemgent) in DMSO to obtain a stock concentration of 5 $\mathrm{mmol} / \mathrm{L}$.

$\underline{\text { Maturation medium }}$

Serum free monolayer differentiation medium

$\underline{\text { MEF medium }}$

$500 \mathrm{ml}$ DMEM (high glucose, GlutaMAX $\left.{ }^{\mathrm{TM}}\right)(61965026$, Gibco)

$120 \mathrm{ml}$ FBS

$6 \mathrm{ml}$ MEM NEAA

$6 \mathrm{ml} \mathrm{P} / \mathrm{S}$

\section{$\underline{\text { mESC medium }}$}

$500 \mathrm{ml}$ DMEM (high glucose, no pyruvate, 25 mmol/1 HEPES) (42430025, Gibco)

$92 \mathrm{ml} \mathrm{FBS}$

$6.2 \mathrm{ml} \mathrm{L-Glutamine} \mathrm{(200} \mathrm{mmol/L;} \mathrm{25030-024,} \mathrm{Invitrogen)}$

$6.2 \mathrm{ml}$ MEM-NEAA (10 mmol/L; 11140-050, Invitrogen)

$6.2 \mathrm{ml}$ Nucleoside mix $(100 \mathrm{X})^{*}$

$5 \mathrm{ml}$ Sodium Pyruvate (100 mmol/L; 11360-039, Invitrogen)

$6.2 \mathrm{ml}$ Penicillin and Streptomycin (P/S: $5.000 \mathrm{U} / \mathrm{ml}$ and $5 \mathrm{mg} / \mathrm{ml}$; 15070-063, Invitrogen)

$4.34 \mu 1$ 2-ß-Mercaptoethanol (31350-010, Sigma)

$62 \mu$ Leukemia inhibitory factor (LIF) $10^{7} \mathrm{U} / \mathrm{ml}$

*Nucleoside mix (100X)

Adenosine $30 \mu \mathrm{mol} / \mathrm{L}$

Guanosine $30 \mu \mathrm{mol} / \mathrm{L}$

Cytidine $30 \mu \mathrm{mol} / \mathrm{L}$ 
Uridine $30 \mu \mathrm{mol} / \mathrm{L}$

Thymidine $10 \mu \mathrm{mol} / \mathrm{L}$

Mesoderm Induction medium

Serum free monolayer differentiation medium

$5 \mathrm{ng} / \mathrm{ml} \mathrm{BMP} 4$

$9 \mathrm{ng} / \mathrm{ml}$ Activin A

$5 \mathrm{ng} / \mathrm{ml} \mathrm{bFGF}$

$1 \mu \mathrm{mol} / \mathrm{L} \mathrm{CHIR}$

Medium containing grow factor supplements are prepared fresh, prior to use.

$\underline{\text { Serum free monolayer differentiation medium }}$

500 ml RPMI 1640 with GlutaMAX ${ }^{\text {TM }}$ (61870, Invitrogen)

$5 \mathrm{ml}$ Sodium Pyruvate

$5 \mathrm{ml} \mathrm{P} / \mathrm{S}$

All reagents are thoroughly mixed and stored at $4{ }^{\circ} \mathrm{C}$ for 2 weeks. $2 \%$ B27

supplement (17504-044, Invitrogen) is added prior to use.

$\underline{\text { Selection medium }}$

49.15 ml RPMI 1640 [-glucose, -glutamine] (01-101-1A; Biological Industries/WKS

Labordiagnostik,)

$0.25 \mathrm{ml}$ sodium lactate (71723, Sigma) [Final concentration: $2.2 \mathrm{mmol} / \mathrm{L}$ ]

$0.5 \mathrm{ml} \mathrm{P} / \mathrm{S}$

$0.1 \mathrm{ml}$ 2-mercaptoethanol (50 mmol/L; 31350010; Invitrogen) [final concentration:

$0.1 \mathrm{mmol} / \mathrm{L}]$

TeSR ${ }^{\text {TM}}-E 8^{\text {TM }}$ medium

Make up TeSR ${ }^{\text {TM}}-E 8$ medium (05940, StemCell Technologies) according to manufacturers protocol, including $1 \% \mathrm{P} / \mathrm{S}$.

\section{$\underline{10 X}$ DMEM}

$669 \mathrm{mg}$ DMEM powder (52100-039, Invitrogen) dissolved in $5 \mathrm{ml}$ sterile $\mathrm{H}_{2} \mathrm{O}$.

Filter the solution through a $0.22 \mu \mathrm{m}$ syringe filter. Stored at $4{ }^{\circ} \mathrm{C}$ for $1-2$ months. 


\section{X DMEM}

$1 \mathrm{ml} \quad$ 10X DMEM

$120 \mathrm{ml} \quad$ FBS

$100 \mu \mathrm{l} \quad$ L-glutamine

$100 \mu \mathrm{l} \quad \mathrm{P} / \mathrm{S}$

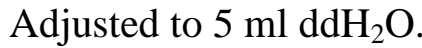




\section{$\underline{\text { Reagents and solutions for isometric force measurements: }}$}

For making Tyrode's working solution, the following stock solutions were prepared:

\section{$\underline{\mathrm{CaCl}}_{2}$ stock $(2.25 \mathrm{M})$}

$165.57 \mathrm{~g} \quad \mathrm{CaCl}_{2} \times 2 \mathrm{H}_{2} \mathrm{O}(\mathrm{MW}=147.02)$

$500 \mathrm{ml} \quad \mathrm{ddH}_{2} \mathrm{O}$

$\underline{\mathrm{MgCl}_{2}} \underline{\text { stock }(1.05 \mathrm{M})}$

$106.83 \mathrm{~g} \quad \mathrm{MgCl}_{2} \times 6 \mathrm{H}_{2} \mathrm{O}(\mathrm{MW}=203.01)$

$500 \mathrm{ml} \quad \mathrm{ddH}_{2} \mathrm{O}$

$\underline{\text { Stock I }}\left(\mathrm{Ca}^{2+}\right.$ concentration in stock I can be adjusted accordingly).

$\begin{array}{llll}\text { Tyrode }\left[\mathrm{Ca}^{2+}\right]: & 1.8 \mathrm{mM} & 0.4 \mathrm{mM} & 0.2 \mathrm{mM}\end{array}$

$\mathrm{NaCl}(\mathrm{MW}=58.44): \quad 175 \mathrm{~g} \quad 175 \mathrm{~g} \quad 175 \mathrm{~g}$

$\mathrm{KCl}(\mathrm{MW}=74.56): \quad 10 \mathrm{~g} \quad 10 \mathrm{~g} \quad 10 \mathrm{~g}$

$\mathrm{CaCl}_{2}$ stock $(2.25 \mathrm{M})$ : $\quad 20 \mathrm{ml} \quad 4.44 \mathrm{ml} \quad 2.22 \mathrm{ml}$

$\mathrm{MgCl}_{2}$ stock $(1.05 \mathrm{M}): \quad 25 \mathrm{ml} \quad 25 \mathrm{ml} \quad 25 \mathrm{ml}$

Fill with $\mathrm{ddH}_{2} \mathrm{O}$ : $\quad 1000 \mathrm{ml} \quad 1000 \mathrm{ml} \quad 1000 \mathrm{ml}$

$\underline{\text { Stock II }}$

$50 \mathrm{~g} \quad \mathrm{NaHCO}_{3}(\mathrm{MW}=84.01)$

$1000 \mathrm{ml} \quad \mathrm{ddH}_{2} \mathrm{O}$

$\underline{\text { Stock III }}$

$5.8 \mathrm{~g} \quad \mathrm{NaH}_{2} \mathrm{PO}_{4}(\mathrm{MW}=137.99)$

$1000 \mathrm{ml} \quad \mathrm{ddH}_{2} \mathrm{O}$

All stock solutions can be stored after reconstitution at $4{ }^{\circ} \mathrm{C}$ until required. 
$\underline{\text { Tyrode's Working Solution }}$

$\begin{array}{lllll}\text { Stock I: } & 40 \mathrm{ml} & 80 \mathrm{ml} & 120 \mathrm{ml} & 200 \mathrm{ml} \\ \text { Stock II: } & 38 \mathrm{ml} & 76 \mathrm{ml} & 114 \mathrm{ml} & 140 \mathrm{ml} \\ \text { Stock III: } & 10 \mathrm{ml} & 20 \mathrm{ml} & 30 \mathrm{ml} & 50 \mathrm{ml} \\ \text { Glucose: } & 1 \mathrm{~g} & 2 \mathrm{~g} & 3 \mathrm{~g} & 5 \mathrm{~g} \\ \text { Ascorbic acid: } & 50 \mathrm{mg} & 100 \mathrm{mg} & 150 \mathrm{mg} & 250 \mathrm{mg} \\ \mathrm{ddH}_{2} \mathrm{O}: & 1 \mathrm{~L} & 2 \mathrm{~L} & 3 \mathrm{~L} & 5 \mathrm{~L}\end{array}$

Prior to isometric force measurements, Tyrode's working solutions were mixed (as indicated above), dissolved thoroughly and poured into thermostatted force transducer reservoirs as measurement buffer. All stock solutions were stored at $4{ }^{\circ} \mathrm{C}$ until needed.

\section{Immunoblotting reagents:}

$\underline{6 X \text { Laemmli loading buffer }}$

$3 \mathrm{ml} \quad$ Tris-HCL $0.5 \mathrm{M}(\mathrm{pH} 6.8)$

$0.75 \mathrm{ml} \quad$ Bromophenol blue $(0.5 \%)$

$1.2 \mathrm{~g} \quad \mathrm{SDS}$

$1.5 \mathrm{ml} \quad$ Glycerin (100\%)

Fill to $9 \mathrm{ml} \quad \mathrm{ddH}_{2} \mathrm{O}$

Aliqout $900 \mu \mathrm{l}$ into tubes. Add $150 \mu 1$ 2-ß-Mercaptoethanol and store at $-20{ }^{\circ} \mathrm{C}$ until use.

\section{$\underline{0.5 \mathrm{M} \text { Tris-HCL pH } 6.8}$}

$30.28 \mathrm{~g} \quad$ Tris-HCl

$400 \mathrm{ml} \quad \mathrm{H}_{2} \mathrm{O}$

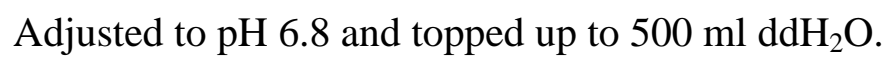

$\underline{1.5 \mathrm{M} \text { Tris-HCL } \mathrm{pH} 8.8}$

$90.85 \mathrm{~g} \quad$ Tris-HCl

$400 \mathrm{ml} \quad \mathrm{ddH}_{2} \mathrm{O}$

Adjusted to $\mathrm{pH} 8.8$ and topped up to $500 \mathrm{ml} \mathrm{H}_{2} \mathrm{O}$. 
$\underline{\text { SDS-PAGE gel preparation }}$

$\underline{\text { Separating gel }}$

\begin{tabular}{|l|l|l|l|l|l|l|l|}
\hline $\begin{array}{l}\text { Volume (ml) } \\
\text { (2 Gels) }\end{array}$ & $\mathbf{5 \%}$ & $\mathbf{6 \%}$ & $\mathbf{8 \%}$ & $\mathbf{1 0 \%}$ & $\mathbf{1 2 \%}$ & $\mathbf{1 5 \%}$ & $\mathbf{1 8 \%}$ \\
\hline KDa & $220-60$ & $150-50$ & $105-40$ & $90-25$ & $60-20$ & $45-10$ & $40-4$ \\
\hline $\mathbf{H}_{\mathbf{2}} \mathbf{O}$ & 5,6 & 5,3 & 4,6 & 4,0 & 3,3 & 2,3 & 1,3 \\
\hline $\mathbf{3 0} \%$ Acrylamide & 1,7 & 2,0 & 2,7 & 3,3 & 4,0 & 5,0 & 6,0 \\
\hline $\mathbf{1 , 5}$ M Tris pH 8,8 & 2,5 & 2,5 & 2,5 & 2,5 & 2,5 & 2,5 & 2,5 \\
\hline $\mathbf{1 0} \%$ SDS & 0,1 & 0,1 & 0,1 & 0,1 & 0,1 & 0,1 & 0,1 \\
\hline $\mathbf{1 0} \%$ APS & 0,1 & 0,1 & 0,1 & 0,1 & 0,1 & 0,1 & 0,1 \\
\hline TEMED & 0,01 & 0,008 & 0,006 & 0,004 & 0,004 & 0,004 & 0,004 \\
\hline
\end{tabular}

Stacking/collecting gel

\begin{tabular}{|l|l|l|}
\hline ml & 4 gels & 2 gels \\
\hline $\mathbf{H}_{2} \mathbf{O}$ & 5,6 & 2,8 \\
\hline $\mathbf{3 0} \%$ Acrylamid & 0,85 & 1,7 \\
\hline $\mathbf{0 , 5}$ M Tris pH 6,8 & 2,5 & 1,25 \\
\hline $\mathbf{1 0} \%$ SDS & 0,1 & $0,05(50 \mathrm{ul})$ \\
\hline $\mathbf{1 0} \%$ APS & 0,1 & $0,05(50 \mathrm{ul})$ \\
\hline TEMED & 0,01 & $0,005(5 \mathrm{ul})$ \\
\hline
\end{tabular}

$\underline{\text { PVDF membrane preparation }}$

PVDF membranes were immersed in $100 \%$ Methanol for 10 seconds. Next, membranes were washed in $\mathrm{ddH} 2 \mathrm{O}$ for 5 minutes and placed in transfer buffer and allowed to equilibrate for 10 minutes. Thereafter, PVDF membranes were activated for SDS gel blot transfers.

\section{$\underline{10 X}$ Transfer Buffer}

$\begin{array}{ll}60.5 \mathrm{~g} & \text { Tris-Base } \\ 288 \mathrm{~g} & \text { Glycine }\end{array}$

Dissolve thoroughly in $2 \mathrm{~L} \mathrm{ddH}_{2} \mathrm{O}$. 


\section{$\underline{1 X \text { Transfer Buffer }}$}

$200 \mathrm{ml} \quad$ 10x Buffer

$400 \mathrm{ml} \quad$ Methanol

Adjusted to $2 \mathrm{~L} \mathrm{ddH}_{2} \mathrm{O}$.

\section{Ponceau S Solution}

Ponceau S-solution (Applichem) is used neat for staining of western blot membranes. Membranes were incubated in Ponceau-S solution for 1-5 minutes in RT and then rinsed with ddH2O until the membrane background turned white and the stained bands were clearly contrasted. After image documentation, the ponceau S stains were rinsed away in TBST.

\section{$\underline{10 X}$ TBS stock solution}

\section{$121.14 \mathrm{~g} \quad$ Tris-Base}

$175.32 \mathrm{~g} \quad \mathrm{NaCl}$

Add all reagents and $1.5 \mathrm{~L}$ of $\mathrm{ddH}_{2} \mathrm{O}$ in a suitable glass beaker and mix until all reagents were dissolved. Equilibrate the solution with fuming $\mathrm{HCl}$ till $\mathrm{pH}$ 7.5, then fill up to $2 \mathrm{~L}$ with $\mathrm{ddH}_{2} \mathrm{O}$.

\section{$\underline{1 X}$ TBS solution}

$\begin{array}{ll}100 \mathrm{ml} & 10 x \text { TBS } \\ 900 \mathrm{ml} & \mathrm{H}_{2} \mathrm{O} \\ 1 \mathrm{ml} & \text { Tween } 20\end{array}$

Mix all reagents until they dissolve. Tween 20 is extremely viscous and requires approximately $10-15$ minutes to dissolve completely.

\section{X Electrophoresis (Running) Buffer (pH 8.3 -8.7)}

$\begin{array}{ll}60.6 \mathrm{~g} & \text { Tris- Base } \\ 288 \mathrm{~g} & \text { Glycine } \\ 20 \mathrm{~g} & \text { SDS }\end{array}$

Dissolve all reagents in $1 \mathrm{~L} \mathrm{ddH}_{2} \mathrm{O}$ and topped up to $2 \mathrm{~L} \mathrm{ddH}_{2} \mathrm{O}$ and mix thoroughly. During gel electrophoresis, 1: $10 \mathrm{ml}$ dilution with $\mathrm{ddH}_{2} \mathrm{O}$ is prepared fresh, to obtain $1 \mathrm{X}$ running buffer. 


\section{DNA Gel Electrophoresis:}

\section{$\underline{50 X \text { TAE }}$}

$242 \mathrm{~g} \quad$ Tris-Base

$100 \mathrm{ml} \quad$ EDTA $(0.5 \mathrm{M} \mathrm{pH} \mathrm{8.0)}$

$57.1 \mathrm{ml} \quad$ Glacial Acetic Acid

Dissolve first in $800 \mathrm{ml}$, pH 8.5 adjusted and fill up to $1 \mathrm{~L} \mathrm{H}_{2} \mathrm{O}$. Gel electrophoresis run is diluted $1: 50$ to obtain $1 \mathrm{X}$ TAE gel running buffer prior to use.

\section{$\underline{1.5 \% \text { Agarose gel }}$}

$1.5 \mathrm{~g}$ of agarose powder (Applichem) is dissolved in $100 \mathrm{ml}$ 1XTAE buffer and boiled to obtain a $1 \%$ agarose gel solution. Following cooling of the solution, 0.2 $\mu \mathrm{g} / \mathrm{ml}$ ethidium bromide (EtBR) is added and swirled to obtain a homogenous solution. The solution is then poured into a casting tray with appropriate combs and allowed to solidify.

6X DNA loading buffer (2 coloured)

$\begin{array}{ll}75 \mathrm{mg} & \text { Bromophenol-Blue } \\ 25 \mathrm{mg} & \text { Xylene cyanol } \\ 100 \mathrm{ml} & \text { Glycerol (30\%) }\end{array}$

All reagents are mixed thoroughly and can be stored in $4{ }^{\circ} \mathrm{C}$ indefinitely. 


\section{$\underline{\text { Reagents for Immunohistochemistry/Flow cytometry: }}$}

Blocking (permeabilization) Buffer

$500 \mathrm{ml}$ 1X PBS (0.05 M, pH 7.4, Invitrogen)

$25 \mathrm{ml} \mathrm{FBS}$

$5 \mathrm{~g}$ Bovine Serum Albumin (BSA) (Sigma, A3311)

$2.5 \mathrm{ml}$ Triton X-100 (Sigma, T8787)

All reagents are mixed together thoroughly. Triton X-100 is viscous and requires constant stirring to dissolve. Blocking buffer can be aliquoted and stored at $-20{ }^{\circ} \mathrm{C}$. 